\title{
Elucidating Mechanisms of Neurobehavioral Dysfunction in a Rodent Model of Traumatic Brain Injury
}

Aric F. Logsdon

Follow this and additional works at: https://researchrepository.wvu.edu/etd

\section{Recommended Citation}

Logsdon, Aric F., "Elucidating Mechanisms of Neurobehavioral Dysfunction in a Rodent Model of Traumatic Brain Injury" (2016). Graduate Theses, Dissertations, and Problem Reports. 6106.

https://researchrepository.wvu.edu/etd/6106

This Dissertation is protected by copyright and/or related rights. It has been brought to you by the The Research Repository @ WVU with permission from the rights-holder(s). You are free to use this Dissertation in any way that is permitted by the copyright and related rights legislation that applies to your use. For other uses you must obtain permission from the rights-holder(s) directly, unless additional rights are indicated by a Creative Commons license in the record and/ or on the work itself. This Dissertation has been accepted for inclusion in WVU Graduate Theses, Dissertations, and Problem Reports collection by an authorized administrator of The Research Repository @ WVU.

For more information, please contact researchrepository@mail.wvu.edu. 


\title{
Elucidating Mechanisms of Neurobehavioral Dysfunction in a Rodent Model of Traumatic Brain Injury
}

\author{
Aric F. Logsdon \\ Dissertation submitted \\ to the School of Pharmacy \\ at West Virginia University \\ in partial fulfillment of the requirements for the degree of \\ Doctor of Philosophy in \\ Pharmaceutical \& Pharmacological Sciences \\ Jason D. Huber, Ph.D., Chair \\ Charles L. Rosen, M.D., Ph.D. \\ Rae R. Matsumoto, Ph.D. \\ Diane B. Miller, Ph.D. \\ James A. Bibb, Ph.D. \\ Department of Pharmaceutical \& Pharmacological Sciences \\ Robert C. Byrd Health Sciences Center
}

Morgantown, West Virginia

2016

Keywords: Traumatic brain injury, blood-brain barrier, endoplasmic reticulum stress, neuroinflammation, neurobehavioral dysfunction

Copyright 2016 Aric F. Logsdon 


\title{
ABSTRACT \\ Elucidating Mechanisms of Neurobehavioral Dysfunction in a Rodent Model of Traumatic Brain Injury
}

\author{
Aric F. Logsdon
}

Neuropsychiatric symptoms and cognitive deficits are common among victims of a traumatic brain injury (TBI), and currently, there are no effective treatments to improve outcome. We first developed a clinically-relevant blast TBI model based on lung scaling parameters to elucidate mechanisms of neuronal cell death. In order to discover effective treatments to improve outcome, we had to validate our novel preclinical model of TBI. TBI is an external force that can cause damage to the neurovascular unit (NVU), which can lead to secondary effects, cell death and behavioral dysfunction. In our first study we observed that our model damaged the NVU, increased neuronal cell death, and produced cognitive deficits in young adult Sprague-dawley rats. The link between damage to the NVU and neurobehavioral dysfunction following TBI is poorly understood. Recently secondary injury cascades, such as endoplasmic reticulum (ER) stress and neuroinflammation, have been hypothesized to be early indicators for the development of neurobehavioral dysfunction. Therefore, we examined the regional and temporal profile of these secondary injury cascades using our validated rodent TBI model. We also measured neurobehavioral dysfunction using a variety of functional assays at various time points post-TBI. Tissues from brain regions associated with the behavioral sequelae of TBI were evaluated for biochemical changes. Furthermore, we investigated the neurophysiological response in brain slice recordings at various time points after TBI. We discovered that TBI produced spatial memory deficits in the rats and altered synaptic firing rates in the hippocampus. In our next study, we revealed a robust increase in markers of ER stress and neuroinflammation within the frontal cortex after TBI. Interestingly, we observed impulsive-like behavior in rats after TBI, which is indicative of damage to the frontal cortex. After characterization of the injury response, we investigated the role of ER stress modulation in mediating secondary injury cascades and neurobehavioral dysfunction following TBI. Salubrinal, an ER stress modulator, attenuated markers of neuroinflammation and neuronal cell death. Most importantly, ER stress modulation ameliorated impulsive-like behavior in rats after TBI. The final portion of this study was to elucidate a link between ER stress and the development of Chronic Traumatic Encephalopathy (CTE). We revealed a potential link between repetitive blast injury and neuropsychiatric symptoms associated with CTE. Tau phosphorylation and aggregation are considered hallmarks of CTE development. We observed an increase in marker of tau phosphorylation and conformational change in rats exposed to repetitive blast. We also observed spatial memory deficits and impulsive-like behavior after repetitive TBI. Together, these results suggest that repetitive blast exposure may lead to tauopathy and the behavioral sequelae associated with CTE. Future studies should aim to causally link secondary injury cascades to tauopathy in order to elucidate new drug targets to improve patient outcome after TBI. 
This work is dedicated to the support of my Family

Mark Logsdon

Laurie Logsdon

Richard Logsdon

Paula Logsdon

Richard Astor

Linda Astor 


\section{Acknowledgements}

The work presented in this dissertation could not have been possible without the support and guidance of many individuals including my mentors, colleagues and friends.

First and foremost, I must thank my loving fiancée, Kimberly Alonge. She has helped me to find my passion for science. She has also been there for me when science was tough and helped me to prevail. Kim provided me with the strength to persevere during the trials of graduate school and constantly reminded me that I would succeed.

I would like to thank Jason Huber for teaching what it takes to be a scientist and how to write like a scientist. His mentorship was straightforward and his input on manuscript preparation was by far the best advice ever given. Also, thank you for introducing me to the incredible Barriers of the CNS group

I would like to thank Charles Rosen for his incredible insight into the purpose of our work and for offering an invaluable clinical perspective to our findings. I would like to thank Xinian Li and Zhenjun Tan for teaching me proper animal handling and care. I am forever grateful to them for teaching me the art of Stroke surgery, Evan's blue analysis, and for showing me how to record physiological changes. In addition, I would also like to thank Penny Humberson for making sure the lab was always in good shape and for always being there for me when times were tough. Thank you all!

I want to thank Diane Miller for offering valuable equipment and excellent guidance during the $\mathrm{PhD}$ process especially with the behavioral analysis. I also want to thank James O'Callaghan for his expertise of glial reactivity and inflammation. Every lab meeting was full of great ideas and discussion.

I would like to thank Jim Simpkins for his guidance and support. In particular for his help in writing manuscripts and planning experiments. I would like to thank Paul Lockman for his guidance and support upon his arrival to WVU. In particular for his help in data analysis and experimental design. In addition, I would also like to thank these two mentors for their encouraging words and the reference letters sent in my regard. Thank you both for taking me under your wing when I was unsure of my future plans.

I would like to thank James Bibb, Florian Plattner and Adan Hernandez for their guidance into the art of biochemistry and electrophysiology. I wish to thank them for their southern hospitality during my visit.

I would like to thank Rae Matsumoto and Linda Nguyen for help with behavioral design and statistical analysis. In addition, I would especially like to thank Rae for offering space, equipment and supplies during my early years of training, also for inviting me to her Christmas Parties every year!

I would like to thank Ryan Turner, Matthew Robson and Zach Naser for their help early on during my $\mathrm{PhD}$ training. With their help I learned valuable skills that were required to acquire data for my dissertation. Without the help of these three, I would still be trying to learn how to run a Western blot...

I would especially like to thank Brandon Lucke-wold for keeping me motivated over the past few years. His drive is unmatched and his work ethic is insurmountable. Our ability to work as a team, has made us successful graduate students and ultra-productive. I hope we can work together far into our careers.

If anyone was left out of these acknowledgements just know that I am truly grateful for all of your help. Thank you all for the guidance and support throughout this rigorous process of $\mathrm{PhD}$ training! 


\section{Table of Contents}

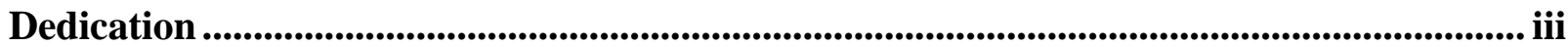

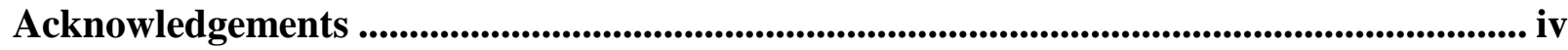

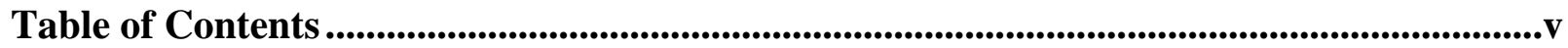

Index of Figures...................................................................................................................................... viii

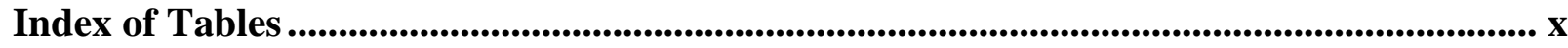

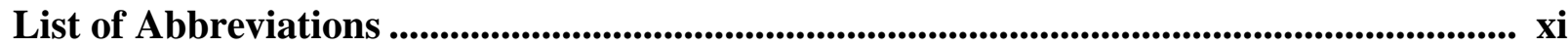

\section{Chapter 1: Introduction}

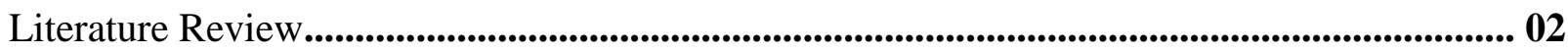

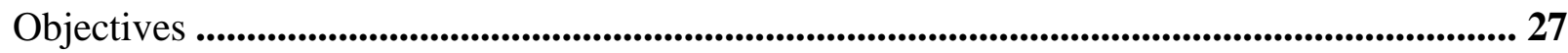

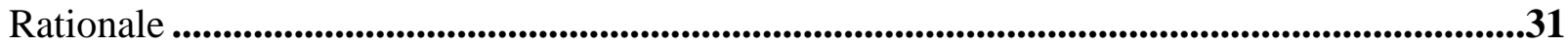

Chapter 2: Validation of neural injury in the rat after traumatic brain injury

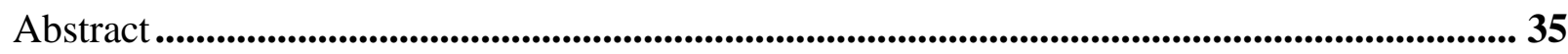

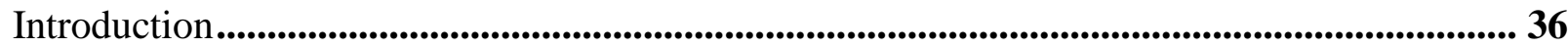

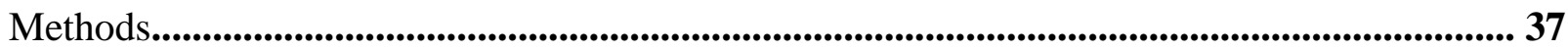

Results

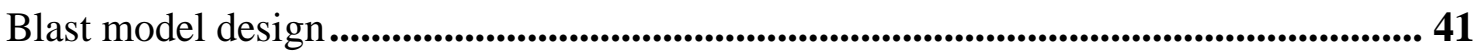

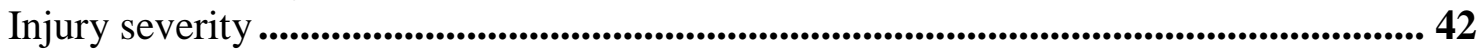

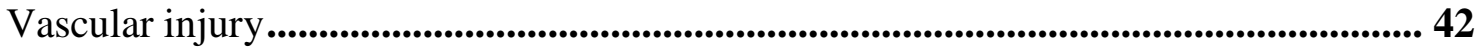

Neurodegeneration ........................................................................................................... 43

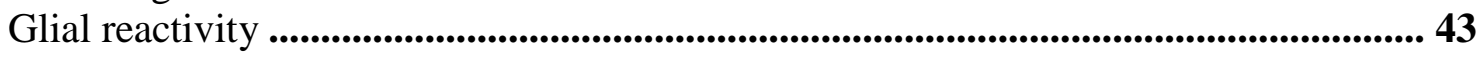

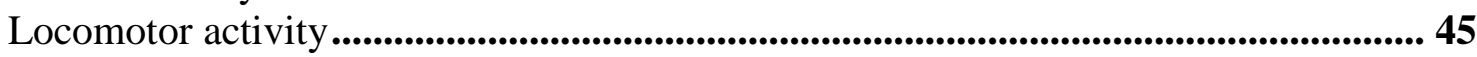

Discussion ..................................................................................................................................................... 46

\section{Chapter 3: Characterization of neural injury and neurobehavioral} dysfunction in the rat after traumatic brain injury

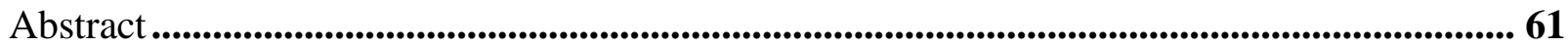

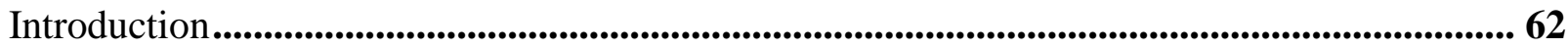

Methods............................................................................................................................................... 63

Results

Neuropathological effects ..................................................................................................... 68

Neurophysiological effects .................................................................................................... 70

Excitotoxic effects ............................................................................................................. 72

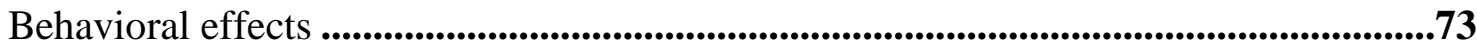

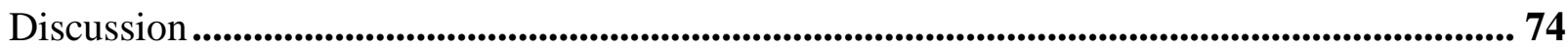




\section{Chapter 4: Endoplasmic reticulum stress modulation ameliorates impulsive- like behavior in rats exposed to traumatic brain injury}

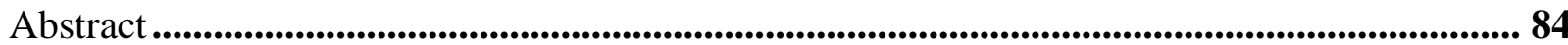

Introduction .............................................................................................................................................. 85

Methods......................................................................................................................................................... 87

Results

Contra coup vascular disruption ............................................................................................... 94

Blast upregulates stress response markers .................................................................95

Salubrinal attenuates ER stress markers ................................................................. 96

Salubrinal modulates ER-mediated apoptosis .......................................................................97

Salubrinal mitigates Caspase-3 cleavage ........................................................................................99

Impulsive-like behavior ameliorated by salubrinal............................................................. 100

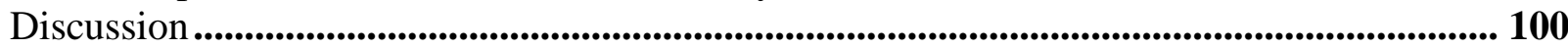

\section{Chapter 5: Salubrinal reduces neuroinflammation and risk-taking behavior in rats exposed to repetitive traumatic brain injury}

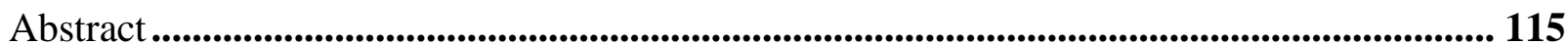

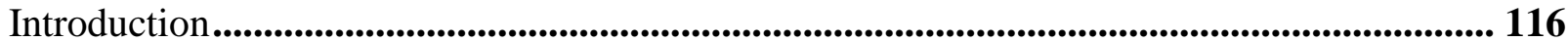

Methods....................................................................................................................118

Results

SAL reduces oxidative stress ................................................................................................... 123

SAL attenuates neurodegeneration ............................................................................................... 124

SAL mitigates neuroinflammation............................................................................................... 124

ER stress and neuroinflammation markers colocalize ....................................................... 126

SAL attenuates neuroinflammation after rTBI ................................................................ 126

SAL ameliorates risk-taking behavior after rTBI .....................................................................126

Mechanism of blast injury .............................................................................................127

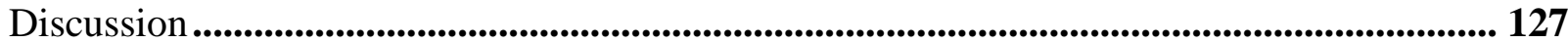

Chapter 6: The quest to model chronic traumatic encephalopathy: a multiple model and injury paradigm experience

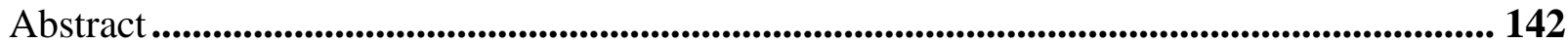

Introduction ...........................................................................................................................................143

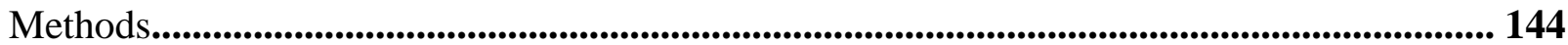

Results

Neurotrauma induces tau hyperphosphorylation ................................................................... 149

Hyperphosphorylation of tau in perivascular regions ........................................................... 150

Tau conformation changes after neurotrauma .......................................................................151

Neurotrauma produces cognitive impairments .................................................................. 152

Impulsivity increased following neurotrauma ...................................................................... 154

Discussion ............................................................................................................................................ 155 


\section{Chapter 7: Modeling Chronic Traumatic Encephalopathy: The Way Forward for Future Discovery}

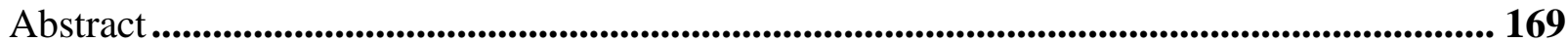

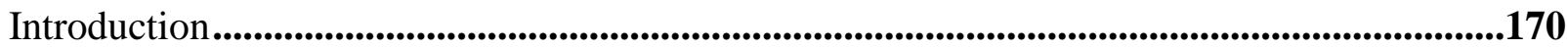

The quest for the ideal model..............................................................................................................171

What are the proper controls for studying CTE? ..................................................................... 172

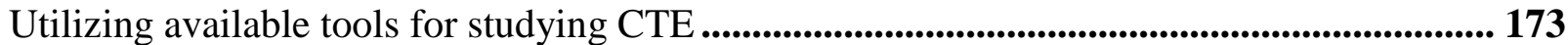

Results from prior CTE modeling studies ............................................................................ 174

Learning from past shortcomings with TBI models ...................................................................... 178

Key questions going forward ........................................................................................................... 180

Inter-injury interval .................................................................................................................. 180

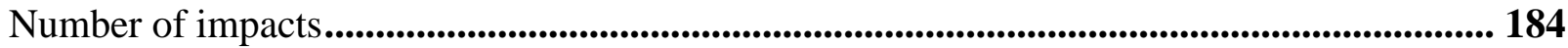

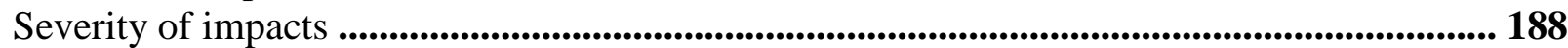

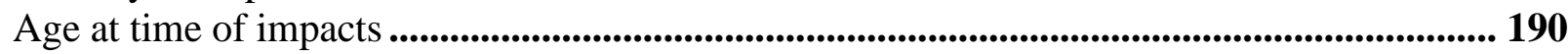

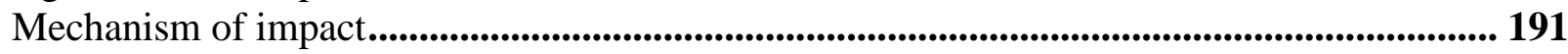

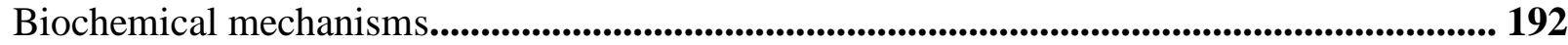

Role of genetics..................................................................................................................................192

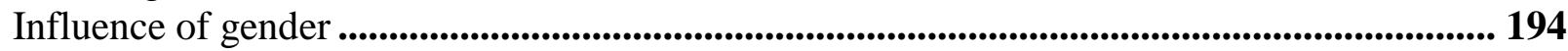

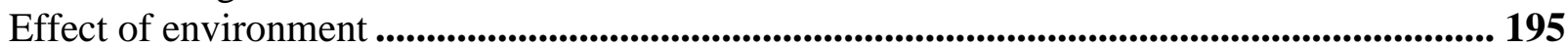

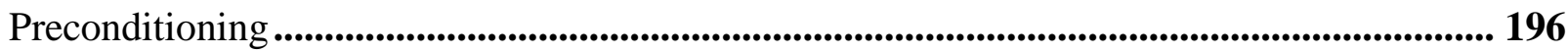

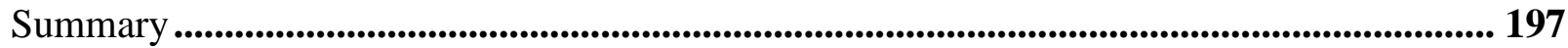

\section{Chapter 8: General Discussion}

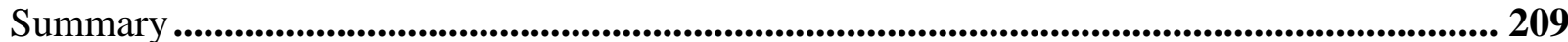

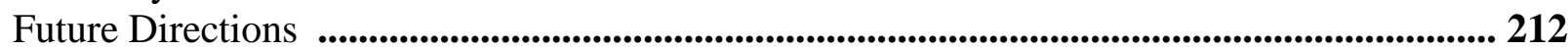

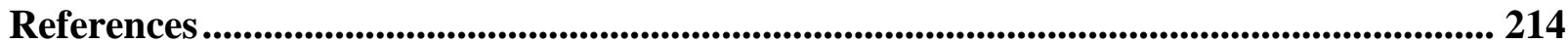




\section{Index of Figures}

\section{Chapter 1}

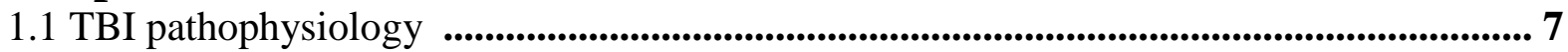

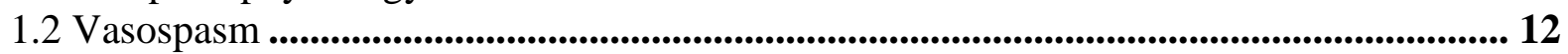

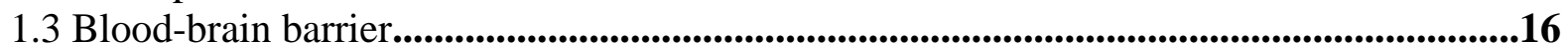

1.4 Secondary mechanism ..........................................................................................................................18

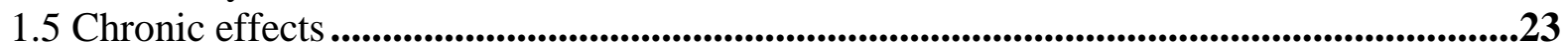

\section{Chapter 2}

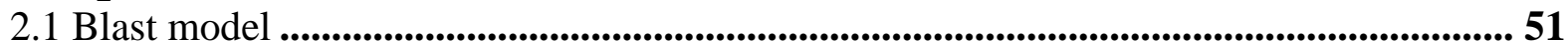

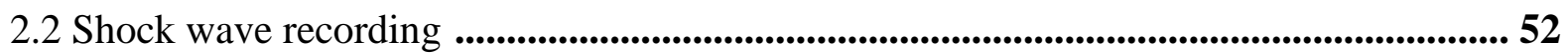

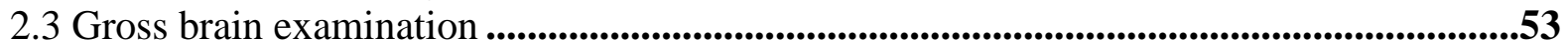

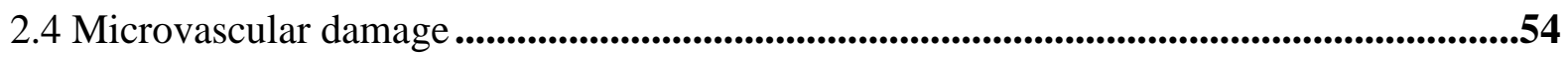

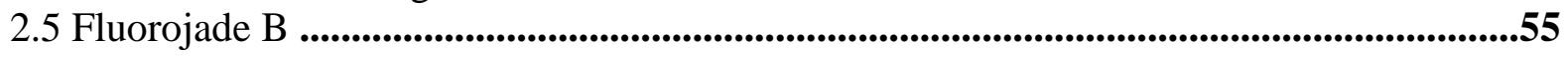

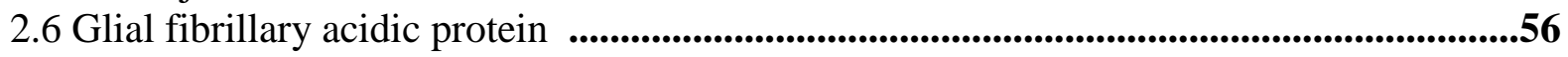

2.7 Ionized calcium-binding adapter molecule-1............................................................................57

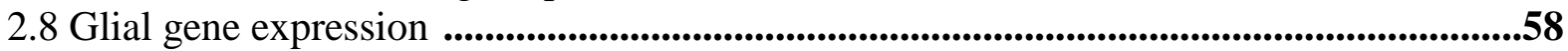

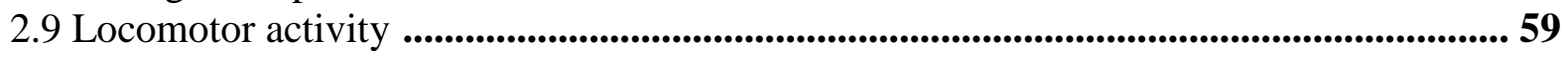

\section{Chapter 3}

3.1 Experimental design................................................................................................................. 77

3.2 Neuropathology global......................................................................................................... 78

3.3 Neuropathology hippocampus .........................................................................................................79

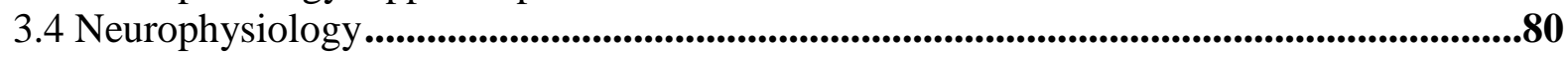

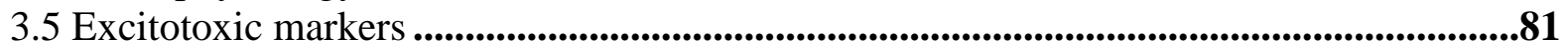

3.6 Fear conditioning and locomotion .................................................................................................82

\section{Chapter 4}

4.1 Schematic of blast injury effects ......................................................................................... 105

4.2 Blood-brain barrier distuption ........................................................................................... 106

4.3 Blast upregulates stress response genes...................................................................................... 107

4.4 SAL attenuates ER stress markers .............................................................................108

4.5 SAL modulates ER-mediated apoptosis ................................................................................109

4.6 SAL reduces CHOP and MAP2 colocalization ...........................................................................110

4.7 CHOP and Caspase-12 colocalization ........................................................................... 111

4.8 SAL reduces apoptosis in left PFC ...................................................................... 112

4.9 SAL ameliorates impulsive-like behavior ...............................................................................113 


\section{Chapter 5}

5.1 Experimental design................................................................................................................... 133

5.2 SAL reduced oxidative stress........................................................................................................... 134

5.3 SAL attenuated neurodegeneration................................................................................................ 135

5.4 SAL mitigated neuroinflammation markers ................................................................................ 136

5.5 BiP and pJNK colocalized with CHOP after rTBI ............................................................... 137

5.6 SAL reduced iNOS, but not GFAP after rTBI........................................................................138

5.7 SAL ameliorated risk-taking behavior after rTBI.................................................................... 139

5.8 Blast injury cascade .......................................................................................................................... 140

\section{Chapter 6}

6.1 Schematic showing experimental design ............................................................................... 158

6.2 Schematic of injury models .............................................................................................................. 159

6.3 Both injury models induce tau hyperphosphorylation ...................................................... 160

6.4 Tau hyperphosphorylation seen in superficial cortex .............................................................. 161

6.5 Both injury models induce tau conformational changes...........................................................162

6.6 Tau conformational markers seen in superficial cortex ......................................................... 163

6.7 Tauopathy seen in perivascular distribution ........................................................................... 164

6.8 Blast injury produces deficits in learning and memory in rats ........................................... 165

6.9 Track plots of spatial acquisition and probe testing................................................................ 166

6.10 Blast injury produces impulsive behavior in rats................................................................... 167

\section{Chapter 7}

7.1 Factors influencing injury outcomes...........................................................................................199

7.2 Methodological challenges of injury paradigms ...........................................................................200 


\section{Index of Tables}

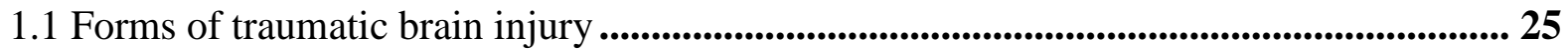

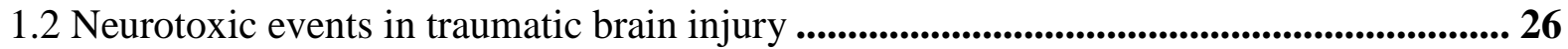

7.1 Chronic traumatic encephalopathy TBI models ..................................................................201

7.2 Shock tube TBI models.............................................................................................................202

7.3 Weight-drop TBI models ....................................................................................................... 203

7.4 TBI models not using craniotomy......................................................................... 204

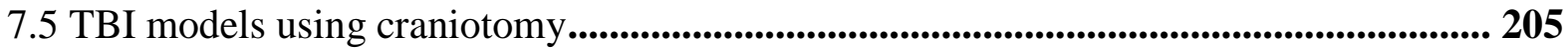

7.6 Other in vivo TBI models.......................................................................................................206

7.7 In vitro and ex vivo TBI models ............................................................................................ 207 


\section{List of Abbreviations}

A $\boldsymbol{\beta}$ : Amyloid Beta

Ach: Acetylcholine

AD: Alzheimer's disease

Akt: Protein kinase B

ANOVA: Analysis of Variance

ApoE: Apolipoprotein E

APP: Amyloid Precursor Protein

ATF4: Activating Transcription Factor 4

ATF6: Activating Transcription Factor 6

BBB: Blood-brain Barrier

BCA: Bicinchoninic Acid

Bcl-2: B-cell Lymphoma 2

BiP: Binding immunoglobulin Protein

bTBI: Blast-induced Traumatic Brain Injury

CHI: Closed Head Injury

CHOP: C/EBP Homology Protein

CNS: Central Nervous System

CONWEP: Use of Conventional Weapons Effects Program

CT: Computerized Tomography

CTCF: Corrected Total Cell Fluorescence

CTE: Chronic Traumatic Encephalopathy

CTEM: chronic traumatic encephalomyelopathy

DAB: 3,3'-Diaminobenzidine

DAPI: 4',6-diamidino-2-phenylindole

DHA: Docosahexaenoic Acid

DMEM: Dulbecco's Modified Eagle Medium

DMSO: Dimethyl Sulfoxide DP: dementia pugilistica

DPBS: Dulbecco's Phosphate Buffered Saline

EB: Evan's Blue

eIF2 $\alpha$ : eukaryotic Initiation Factor 2 alpha

ELISA: Enzyme-linked Immunosorbent Assay

EPM: Elevated Plus Maze

ER: Endoplasmic Reticulum

ERAD: ER-associated Degradation

FJB: Fluorojade B

fMRI: functional Magnetic Resonance Imaging

FST: Forced Swim Test

GADD34: Growth Arrest and Damage-inducible Agent 34

GCS: Glascow Coma Scale

GFAP: Glial Fibrillary Acidic Protein

H\&E: Hematoxylin and Eosin

HDAC: Histone Deacetylase

HRP: Horseradish Peroxidase

IBA1: ionized calcium-binding adapter molecule 1 
IED: Improvised explosive device

IHC: Immunohistochemistry

IKK: Inhibitors of Nuclear Factor Kappa B

IL1及: Interleukin 1 beta

iNOS: inducible Nitric Oxide Synthase

IRE1 $\alpha$ : Inositol Requiring Enzyme 1 alpha

JNK: Janus N-terminal Kinase

kPA: Kilopascal

LTP: Long-term Potentiation

mAB: monoclonal Antibody

MRI: Magnetic Resonance Imaging

MWM: Morris Water Maze

NADPH: nicotinamide adenine dinucleotide phosphate

NF-кB: Nuclear Factor Kappa Beta

NFT: Neurofibrillary Tangle

NMDA: N-methyl-D-aspartate

NOX: NADPH-oxidase

NSE: Neuron-specific Enolase

pAB: polyclonal Antibody

PAS: Photobeam Activity System

PCR: Polymerase Chain Reaction

PD: Parkinson's disease

PERK: Protein Kinase RNA-like Endoplasmic Reticulum Kinase

PET: Positron Emission Tomography

PFC: Prefrontal Cortex

PP1: Protein Phosphatase 1

PSI: Pounds per Square Inch

PTSD: Post-traumatic Stress Disorder

ROS: Reactive Oxygen Species

rTBI: Repetitive Traumatic Brain Injury

S1BF: Somatosensory Barrel Cortex

SAL: Salubrinal

SDS: Sodium Docecyl Sulfate

SEM: Standard Error of the Mean

sTBI: Single Traumatic Brain Injury

TBI: Traumatic Brain Injury

TDP-43: Transactive Response DNA-binding Protein 43

TNFa: Tumor Necrosis Factor alpha

TNT: Trinitrotoluene

TRAF6: TNF Receptor-Associated Factor 6

TUNEL: Terminal Deoxynucleotidyl Transferase-mediated dUTP Nick End Labeling

UPR: Unfolded Protein Response

XBP1: X-box Binding Protein 1 


\section{Chapter One}

\section{Introduction}

This work is published in Compr Physiol. 2015 Jul 1;5(3):1147-60.

doi: 10.1002/cphy.c140057. Review. PMID: 26140712

Logsdon AF*, Lucke-Wold BP*, Turner RC, Huber JD, Rosen CL, Simpkins JW. 


\section{LITERATURE REVIEW}

\section{ABSTRACT}

Traumatic brain injury is acquired from an external force, which can inflict devastating effects to the brain vasculature and neighboring neuronal cells. Disruption of vasculature is a primary effect that can lead to a host of secondary injury cascades. The primary effects of neural injury are rapidly occurring while secondary effects can be activated at later time points and may be more amenable to targeting. Primary effects of brain injury include diffuse axonal shearing, changes in blood brain barrier permeability, and brain contusions. These mechanical events, especially changes to the $\mathrm{BBB}$, can induce calcium perturbations within brain cells producing secondary effects, which include cellular stress, inflammation, and apoptosis. These secondary effects can be potentially targeted to preserve the tissue surviving the initial impact of TBI. In the past, TBI research had focused on neurons without any regard for glial cells and the cerebrovasculature. Now a greater emphasis is being placed on the vasculature and the neurovascular unit following TBI. A paradigm shift in the importance of the vascular response to injury has opened new avenues of drug treatment strategies for TBI. However, a connection between the vascular response to TBI and the development of chronic disease has yet to be elucidated. Long-term cognitive deficits are common amongst those sustaining severe or multiple mild TBIs. Understanding the mechanisms of cellular responses following TBI is important to prevent the development of neuropsychiatric symptoms. With appropriate intervention following TBI, the vascular network can perhaps be maintained and the cellular repair process possibly improved to aid in the recovery of cellular homeostasis. 


\section{INTRODUCTION}

Traumatic brain injury (TBI) represents an enormous societal burden both with regard to prevalence/incidence and economic cost (initial treatment and long-term care due to high morbidity), largely irrespective of initial injury mechanism. In fact, TBI is the leading cause of trauma-related morbidity and mortality in developed countries, with over 55 million people affected internationally (Bruns and Hauser, 2003). Interestingly, the mechanism of TBI sustained is often related to the patient's age with younger patients more likely suffering TBI as the result of motor vehicle accidents, sports, or battlefield exposure to blast waves whereas the elderly population is generally afflicted by falls (neurogenic or cardiogenic in origin). TBI is also distributed bimodally with peak incidences between 15-24 years, and after 75 years of age. Notably, after age 65, patients have increased mortality and worse functional outcome following TBI (Susman et al., 2002). The most prominent cause of TBIs is motor vehicle collisions (Dagher et al., 2010). Concussive injuries are also high amongst professional athletes and the active military due to the high-risk for a neurotraumatic event to occur on the job (Guskiewicz et al., 2003; Rigg and Mooney, 2011). These sub-populations should therefore be the focus for future translational studies.

TBI is unique in that it is always acquired from an external force. One difficulty when treating TBI patients is that we never know when, or how, a TBI will occur. Therefore, the contribution of genetic predisposition and comorbidities to overall post-injury outcomes are hard to ascertain. A valuable scale, called the Glasgow Coma Scale (GCS), is used to assess verbal, motor, and eye-opening responses in order to classify TBI severity (Kay and Teasdale, 2001). The score is based on a 15-point scale. Mild injury correlates with a score of 13+, moderate with a score $9-12$, and severe with a score of $<8$. Its clinical utility is mostly to help determine patient 
status in relation to intracerebral pressure (Balestreri et al., 2004). As such, the clinician can use the score to determine the need for appropriate treatment or management. The values obtained from the GCS help define the severity of TBI and allows clinicians to address the injury more appropriately (Foreman et al., 2007). In addition, patients can display cognitive, emotional, and sensory impairments following mild TBIs and even exhibit physical impairments following more severe forms of TBI. Research now suggests that visible signs, or symptoms, of neurological dysfunction from sports-related TBI may not develop for an extended period of time (Bailes et al., 2013).

Imaging modalities have recently been used to detect some of the subtle injury changes associated with TBI. Microdialysis in combination with nuclear magnetic resonance imaging was used to determine that TBI patients have increased anaerobic metabolism dependent on the pentose phosphate pathway (Jalloh et al., 2014). Diffusion tensor imaging has been used with mixed success in detecting white track lesions following concussion (Shin et al., 2014). Functional magnetic resonance imaging (fMRI) has been used to tease out differences in Salience Network functioning following TBI indicative of failed cognitive control (Jilka et al., 2014). In the clinic, Czosynka's pressure reactivity index can be used to establish a dynamic target for cerebral perfusion pressure (Depreitere et al., 2014). Another important consideration is monitoring of cerebral blood flow to prevent the occurrence of ischemia following TBI. Positron emission tomography, perfusion-weighted MRI, and the perfusion computed tomography scan may be used for this endeavor (Rostami et al., 2014).

Two general forms of TBI exist: closed head injury and penetrating head injury (PeekAsa et al., 2001). TBI is grouped into three levels of severity: mild, moderate and severe (Peskind et al., 2013). Further subclassification of injury is based on symptoms. Subconcussive 
injury can have no initial symptoms, concussive injury (acute) presents with cognitive, emotional, and sleep symptoms, while juvenile head trauma syndrome presents with a lucid interval and unconsciousness. Post-concussive syndrome involves concussive symptoms lasting greater than 3 months, and chronic traumatic encephalopathy involves a several year symptom free stage followed by onset of neuropsychiatric symptoms (McKee et al., 2014). Mild TBIs are common and can go undetected with conventional screening tools, although balance tests and cognitive measurements have been used with varied success rates (Harmon et al., 2013; Ruhe et al., 2014). Typically, mild TBI leaves the subject conscious with mild symptoms; however, rodent students have shown that secondary effects often lead to chronic deficits in cognition, memory, and behavior (Hamm et al., 1992; Milman et al., 2005). Molecular mechanisms of the secondary effects of mild and moderate TBI are being investigated due to the increased awareness of how these effects contribute to chronic neuropsychiatric symptoms (Koponen et al., 2002). Severe TBI is often life-threatening and requires immediate care. Fewer basic scientists study severe TBI because its effects are more likely irreversible in both the short-term and the long-term.

We have limited knowledge of what brain regions are most affected after a TBI, due to the fact the injury can either be focal or diffuse depending on the type and severity. As such, clinicians struggle with identifying which brain regions have been affected after injury. The brain's response to TBI is more often globally distributed with a vast number of dilated perivascular spaces (Inglese et al., 2005). Pathologic changes are scattered throughout the brain as well and are highly dependent on neuroinflammation as evident from gliosis (Streit et al., 2004). With the many different forms of TBI, the diagnosis and treatment plans must be adaptable and situational specific. 
To date, the primary focus of TBI research has been on neurons with little emphasis on glial cells or the cerebrovasculature. Indeed, for this review, we identified 1030 published reports that assess neurons, and only 326 reports that assessed other brain cell types, including 46 reports on brain microvessels. The current skewing of research in TBI literature toward neurons is understandable, given the underlying goal of protecting neurons and preserving brain function. A neurocentric approach however has not resulted in successful translation of therapeutics in TBI (Maas, 2001; Kabadi and Faden, 2014), or other models of neural injury (Korczyn, 2012). It is clear other approaches are needed to be successful. Microvessel disruption plays a substantial role in primary, secondary and chronic effects of TBI, and understanding the response of the vascular system on brain trauma is critical to our ability to effectively treat brain trauma. As such, it is clear other approaches are needed and this review deals with the response of all brain cell types to trauma and focuses on the potential role of microvessel damage to neuronal loss and dysfunction.

\section{Forms of Traumatic Brain Injury}

In human subjects, there are a number of causes of TBI and include motor vehicle collisions, falls, sport-associated injuries, blast exposure, and shaken baby syndrome in infants (Table 2)(Zhang et al., 2014b). Besides characterizing the injury based on a specific inciting event, the injury can be described as open or closed head injury, focal or global injury, and impact or blast in origin. Blast injury can be subdivided further into primary, secondary, tertiary, or quaternary injury, a level of detail beyond the scope of this review but discussed in detail elsewhere in the literature (Covey and Born, 2010; Yeh and Schecter, 2012). The different forms of neurotrauma can further be classified based on clinical features through the GCS assessment, pathological features through advanced imaging assessment, or a combination of the two. 
While numerous causes of neurotrauma exist, TBI is uniformly associated with both primary and secondary injury mechanisms. The primary injury is induced instantaneously by the

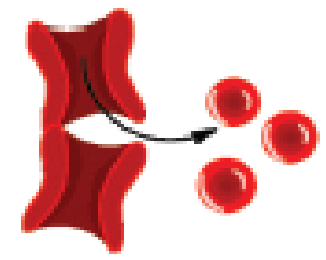

Intracranial pressure spike

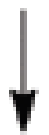

Microvessel rupture

Blood toxin release

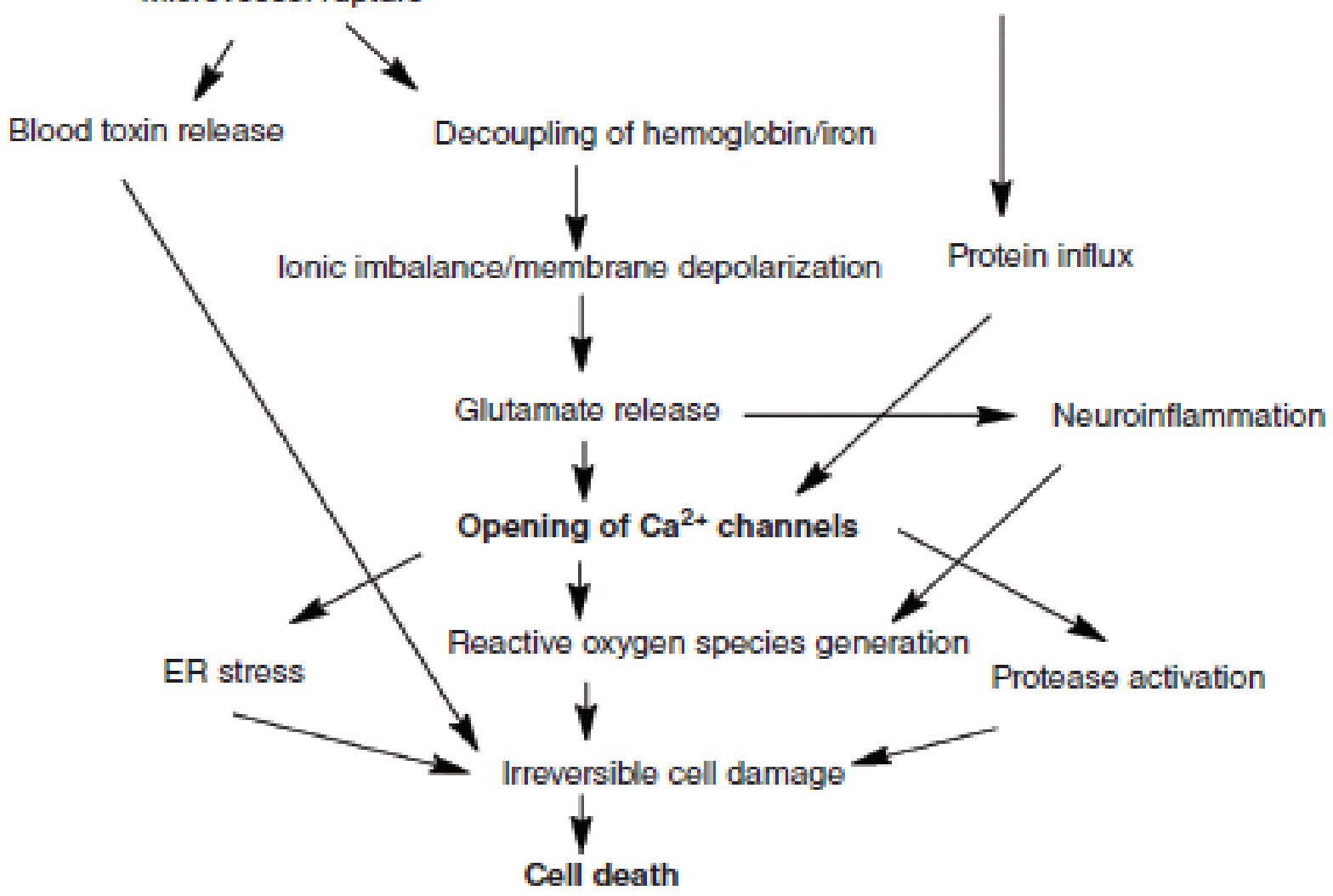

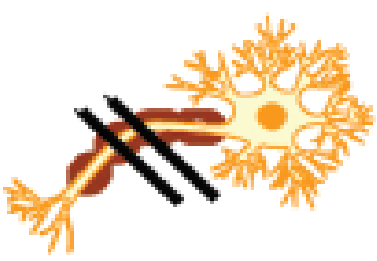

Axonal shearing

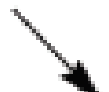

Plasma membrane breakdown

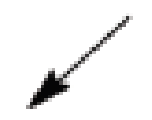

\section{Plasma membrane breakdown}

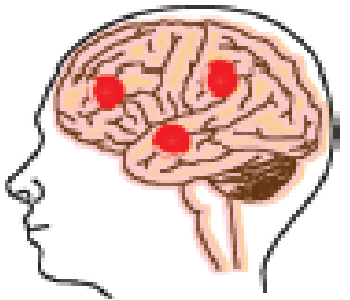

Brain contusion

Figure 1 Pathophysiology of neuronal cell death following traumatic brain injury. An intracranial pressure spike, axonal shearing, and brain contusion contribute to secondary mechanisms that lead to an increase in $\mathrm{Ca}^{2+}$ channel opening. The generation of reactive oxygen species can ultimately contribute to cell death.

application of external forces to the skull and associated brain tissue, depending on the form of TBI. Impact TBI induces focal brain contusions (Sutton et al., 1993), while blast TBI globally 
shears axons (Raghupathi and Margulies, 2002) and damages brain microvessels (Abdul-Muneer et al., 2013) (see Fig 1, Adapted from (Turner et al., 2013a)). The secondary injury follows the primary injury temporally and rodent studies have shown it is associated with induction of signaling cascades that alter metabolic, cellular and molecular events, ultimately leading to alteration in cellular function and/or death (Rink et al., 1995). This period of secondary injury can last from minutes to years and is mediated by biochemical processes that may be targeted by and amenable to therapeutic development (Ramlackhansingh et al., 2011); whereas primary injury can only be prevented (through safety devices, preventative measures, reduced exposure, etc).

To improve understanding of TBI pathophysiology, as well as develop potential therapeutic agents, an array of TBI models have been created and utilized. Some of the most commonly utilized models include the fluid percussion models; variations of the impact acceleration or weight drop models, the controlled cortical impact models, and variations of the shock tube blast models. These models and others such as penetrating injury models are reviewed in depth elsewhere (Albert-Weissenberger and Siren, 2010; Xiong et al., 2013; AngoaPerez et al., 2014; Zhang et al., 2014b). Importantly, TBI represents a highly heterogeneous condition due to the various mechanisms involved and range in forces that are applied/sustained. As such, it is unlikely that one model is adequate for all TBI research and consequently a multifaceted approach is likely required. This is reflected in the approach taken by oversight and advisory groups in which consortiums have been formed to evaluate proposed therapeutics across a range of models in search of identifying not only promising therapeutic candidates but also the most appropriate scenarios in which to clinically test agents (Kochanek et al., 2011). 
Importantly, the diversity of pathophysiological processes involved in neurotrauma has been increasingly recognized with recent studies investigating the role of non-neuronal cell types, such as astrocytes and microglia in neurotrauma, as well as the role of the neurovascular unit and associated blood-brain barrier (BBB). Emerging evidence with in vivo models has implicated these cell types and the BBB in outcomes following neurotrauma, with a particular emphasis on both microvascular and macroscopic components of vascular disruption (Jiang et al., 1992; Pop and Badaut, 2011; McKee et al., 2013; Miyauchi et al., 2013; Yeoh et al., 2013; Liao et al., 2014). This is particularly relevant clinically as macroscopic bleeds, such as epidural and subdural hematomas, may be managed neurosurgically, and microvascular/microscopic bleeds have been implicated in neurodegeneration associated with neurotrauma (Wilberger et al., 1991; McKee et al., 2013).

\section{Traumatic Brain Injury Effects on Neurons}

Following TBI there are immediate primary effects and sub-acute secondary effects. Primary effects to the brain from TBI cause damage to neurons, glia, and the vasculature (Table 3). Secondary effects to the brain from TBI include: inflammatory responses (Begum et al., 2013), cellular stress (Farook et al., 2013), and apoptotic cascades (Clark et al., 1997). The physical forces of TBI can shear axons (Raghupathi and Margulies, 2002), break down

plasmalemma (Cullen et al., 2011), and rupture brain microvessels (Arun et al., 2013). When an axon is torn, or a cell membrane is broken from the external forces of TBI, the neurons can rapidly depolarize and activate voltage gated $\mathrm{Ca}^{2+}$ channels; thereby, increasing intracellular $\mathrm{Ca}^{2+}$ (Gurkoff et al., 2013). Appropriate modeling of the primary effects of TBI will enable researchers to effectively study therapeutic options for the secondary effects. 
Axonal injury has emerged as one of the most important pathological features of TBI. The rotational and acceleration/deceleration components of blast-induced TBI, commonly tears axons apart leading to a robust gliosis response and axonal degeneration (Creed et al., 2011; Goldstein et al., 2012; Li et al., 2013b). Axonal swelling and bulb formation are common morphological hallmarks observed following TBI and contribute to decreased action potential firing (DiLeonardi et al., 2009, 2012). Axonal injury is a result of TBI and is evident in the white matter areas of the brain ( $\mathrm{Li}$ et al., 2013c), and is the initial pathology in neurodegenerative diseases (Adalbert et al., 2009). Injury to axons is present in all severities of TBI and may represent a key hallmark of TBI for modeling purposes (Johnson et al., 2013b). Therefore, defining the neurodegenerative mechanisms induced by axonal injury would allow us to better model the injury and identify specific targets for neuroprotection.

The mechanical forces of brain trauma can also rupture microvessels (Abdul-Muneer et al., 2013). Specifically, the traumatic force causes an intracranial pressure spike which causes cerebral microvessels to burst (Arun et al., 2013). When an external force damages brain microvessels they can release cytotoxic levels of iron into the brain parenchyma (Liu et al., 2013; Nisenbaum et al., 2014). Iron promotes $\mathrm{Ca}^{2+}$-dependent mechanisms, which can stimulate cell survival, or trigger cell death depending on severity and duration of iron exposure (Munoz et al., 2006; Munoz et al., 2011). $\mathrm{Ca}^{2+}$-dependent mechanisms observed following TBI include the unfolded protein response (Ron and Walter, 2007; Farook et al., 2013), proteasomal degradation (Sano and Reed, 2013), autophagy (Bernales et al., 2006), apoptosis (Clark et al., 2000; Nakagawa and Yuan, 2000), and neurodegeneration (Salminen et al., 2009). TBI-induced intracellular $\mathrm{Ca}^{2+}$ increase also prompts reactive oxygen species (ROS) accumulation (Cho et al., 2013), triggers neuroinflammatory cascades (Szmydynger-Chodobska et al., 2010), and can 
influence excitatory amino acids release (Shohami and Biegon, 2014). These secondary effects could potentially be targeted for therapy and warrant further investigation.

\section{Vascular Effects of Traumatic Brain Injury}

The external forces of a TBI induce vascular damage with both the initial insult and the ensuing secondary effects (Roth et al., 2014). It has been proposed that secondary effects can be therapeutically treated because they are driven by pathogenic alterations to signal transduction mechanisms. The vascular effects of TBI include vasospasms, hemorrhage, hypoxia inflammation and BBB disruption. Determining how TBI leads to cell death will provide a better understanding of the secondary effects and provide better therapeutic options. Targets could include BBB restoration after injury, or even using the injury to advantageously deliver drugs during a time window where the BBB is more permeable. This approach will allow pharmacological investigation of mechanisms using drugs that do not easily cross the BBB.

\section{Vasospasms, Subarachnoid Hemorrhage, Edema and Ischemic Hypoxia}

Cerebral vasospasm can be a serious outcome of TBI due to the resulting cerebral hypoperfusion (Izzy and Muehlschlegel, 2014). TBI can trigger molecular mechanisms that rapidly mediate vascular tone (See Fig. 2) (Chen et al., 2013a). One such mechanism is the release of a potent vasoconstrictor, endothelin-1, from damaged pericytes (Dore-Duffy et al., 2011; Paradis and Zhang, 2013). Parenchymal contusions and fever increase the risk for vasospasm (Shahlaie et al., 2011). Up to $68 \%$ of individuals with TBI experience short duration vasospasms (Kramer et al., 2013), which are especially common in soldiers exposed to blast TBI (Alford et al., 2011). 
Vasospasms resulting from TBI can lead to ischemic episodes, energy depletion and cell necrosis; in other words, an inability for brain cells to compensate for increased metabolic functional overload (Ling et al., 2009). Vasospasms are frequently associated with recurrent

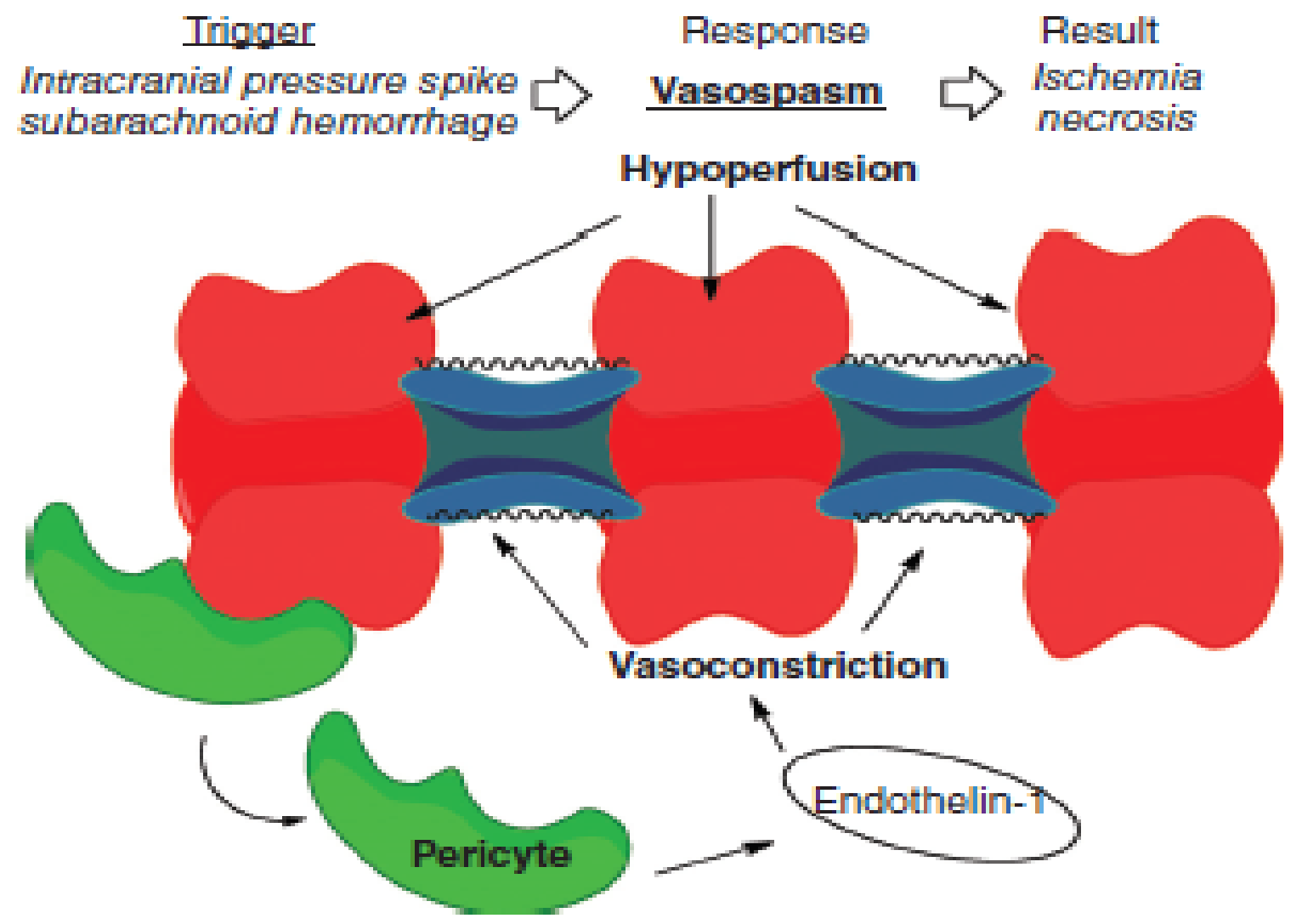

Figure 2 Depiction of a wasospasm resulting from traumatic brain injury. Vasospasm can be friggered by subarachnoid hemorrhage or blood pressure spikes. Pericytes near vessels release endothelin-1, which triggers vasoconstriction.

intracranial hypertension and subarachnoid hemorrhage (Razumovsky et al., 2013; Wilson and Shankar, 2014). Blast TBI, in particular, can cause cerebral vasospasms that last for more than 30 days and are associated with altered mental status (Magnuson et al., 2012). Severe vasospasm must be adequately treated to avoid permanent neurologic deficits or ischemic stroke (Bauer and Rasmussen, 2014). CT angiography is the diagnostic gold standard for vasospasm (Sanelli et al., 
2014). Endovascular treatment and vasodilator therapy have both proven to be successful treatment approaches (Asakuno and Ishida, 2014; Kelman and Reavey-Cantwell, 2014).

Mild TBIs typically exhibit subdural hemorrhaging, while more severe TBIs can display traumatic subarachnoid hemorrhage - increasing the likelihood of diffuse axonal injury, edema, and skull fracture (Mata-Mbemba et al., 2014b). CT imaging is the gold standard for diagnosis of subarachnoid hemorrhage severity (Mata-Mbemba et al., 2014a). The recent establishment of the clinical course score has allowed for the improved monitoring of subarachnoid hemorrhage over time (Brandner et al., 2014), and follow-up CT scans are only needed if new bleeding is a concern (Rubino et al., 2014). TBI has the highest average years of potential life lost for all neurological disorders partly due to traumatic subarachnoid hemorrhage (Rosenbaum et al., 2014). Subarachnoid hemorrhage can lead to increased mortality, extended hospital stays, and central diabetes insipidus (Hochstadter et al., 2014). Most importantly a likely complication of subarachnoid hemorrhage is cerebral vasospasm (Faust et al., 2014). A common treatment option for hemorrhagic change that infiltrates the ventricles is a ventriculostomy followed by ventriculoperitoneal shunt placement (Chalouhi et al., 2014). In severe cases of TBI with subarachnoid hemorrhage where herniation is anticipated, decompressive craniectomy can be performed (Lazaridis and Czosnyka, 2012). Fortunately, most intracerebral hemorrhages are minor and will resolve on their own with time (Levy et al., 2013).

Brain edema following TBI can cause serious complications by limiting brain oxygen delivery and increasing intracranial pressure (Nirula et al., 2014). The pressure increase is more common in children experiencing TBI than adults (Ewing-Cobbs et al., 2000) Hounsfield unit values from brain CT mapped across an intracranial area can be used to accurately predict brain edema following TBI in children (Kim et al., 2014). A new imaging modality, shear wave 
elastography, has also been tested in rodents and gives an accurate representation of edema $(\mathrm{Xu}$ et al., 2014b).Ulinastatin, a serine protease inhibitor, is currently being investigated for reduction of edema in rodents following TBI (Cui and Zhu, 2014). Decompressive craniectomies are common in clinical practice (Nirula et al., 2014). Decompressive craniectomies can prevent the intracranial pressure rise and potential herniation associated with edema (Bor-Seng-Shu et al., 2013). Decompressive cranectomies are not without risk and a recent report showed $83 \%$ of patients developed hygromas and 50\% of patients developed aseptic bone resorption (Pechmann et al., 2014). Continued work is necessary to find viable alternatives for the treatment of edema.

A primary complication of ischemic hypoxia following TBI is hemispheric hypodensity (Foster et al., 2014). Ischemic hypoxia can cause the toxic release of hypoxia-inducible factor 1alpha (Schaible et al., 2014), leading to inflammatory cascades (Qutub and Popel, 2008).

Outcomes from the inflammatory cascade include axonal injury and central myelin damage (van der Eerden et al., 2014). Point in time monitoring revealed that $\sim 10 \%$ of TBI patients experience some ischemic hypoxia (Padayachy et al., 2012). Ischemic hypoxia following TBI is associated with poorer functional independence measures (Cullen et al., 2009). Applying techniques for brain oxygen optimization in clinical care has produced the most successful treatment results (Mangat, 2012). Continuous neurophysiologic recordings are imperative for appropriate patient management (Amantini et al., 2012). In extreme circumstances, red blood cell transfusion may be required (Kramer and Le Roux, 2012). Experimental treatment with mild hypothermia has produced profound preclinical neuroprotection in a variety of TBI models (Fujita et al., 2012a; Miyauchi et al., 2014) and initial human clinical trials (Lee et al., 2010) have proved promising. Blood-brain Barrier Disruption: Neuroinflammation and Reactive Oxygen Species 
The neurovascular unit can become damaged with TBI. The neurovascular unit controls blood flow to the brain, nutrient delivery, and maintenance of BBB integrity (Hartmann et al., 2014). The BBB is an anatomical structure that plays a key role in normal brain physiological regulation. The BBB is composed in part of astrocyte podocytes, a basement membrane, pericytes, and the endothelium connected with tight junction proteins. (Badaut and Bix, 2014). With regard to TBI, little is known about the role of BBB disruption in disease pathology (Alves, 2014).

Neurovascular inflammation can lead to the formation of ROS (Abdul-Muneer et al., 2014). Neuronal injury can occur when ROS exceeds the brain's defense and clearance mechanisms (Adibhatla and Hatcher, 2010). ROS are generated from mitochondrial damage as well as the nicotinamide adenine dinucleotide phosphate-oxidase system at the plasma membrane (Zhang et al., 2012). ROS can eventually contribute to disruption of the BBB, edema, and neuroinflammation (Pun et al., 2009). ROS contribute to downregulation of tight junction proteins at the microvessel interface (Abdul-Muneer et al., 2013). In addition, formation of ROS ultimately triggers glial cell activation producing additive injury to the brain parenchyma (Roth et al., 2014). Oxidative stress stimulates the release of inflammatory factors: interleukin-1beta, tumor necrosis factor-alpha, and transforming growth factor-beta (Abdul-Muneer et al., 2014). ROS also disrupt the vasculatures ability to self-regulate and constrict (DeWitt and Prough, 2009). Ultimately, ROS depression of cerebral metabolism can have long-term consequences manifesting in chronic neurodegeneration and behavioral abnormalities (Tavazzi et al., 2005). Proton therapy through use of hydrogen infused saline can limit the damage to endothelial cells by preventing BBB disruption and the formation of ROS (Ji et al., 2012). Another means of limiting oxidative stress is maintenance of mitochondrial homeostasis (Pandya et al., 2009). 
Uncoupling the mitochondrial oxygen cascade early following TBI has proved beneficial in limiting ROS formation and calcium overload (Pandya et al., 2007). Cerebral microdialysis has been proposed as a diagnostic test for detecting the concentration of free radicals within the brain (Hillered et al., 2005).

BBB disruption is common amongst TBI patients. Approximately $44 \%$ of patients who experience severe TBI have BBB breakdown (Ho et al., 2014). In addition to being effected by ROS, BBB breakdown itself can lead to enhanced neuroinflammation and the formation of ROS (See Fig. 3) (Abdul-Muneer et al., 2014). Damage to the BBB also leads to the release of

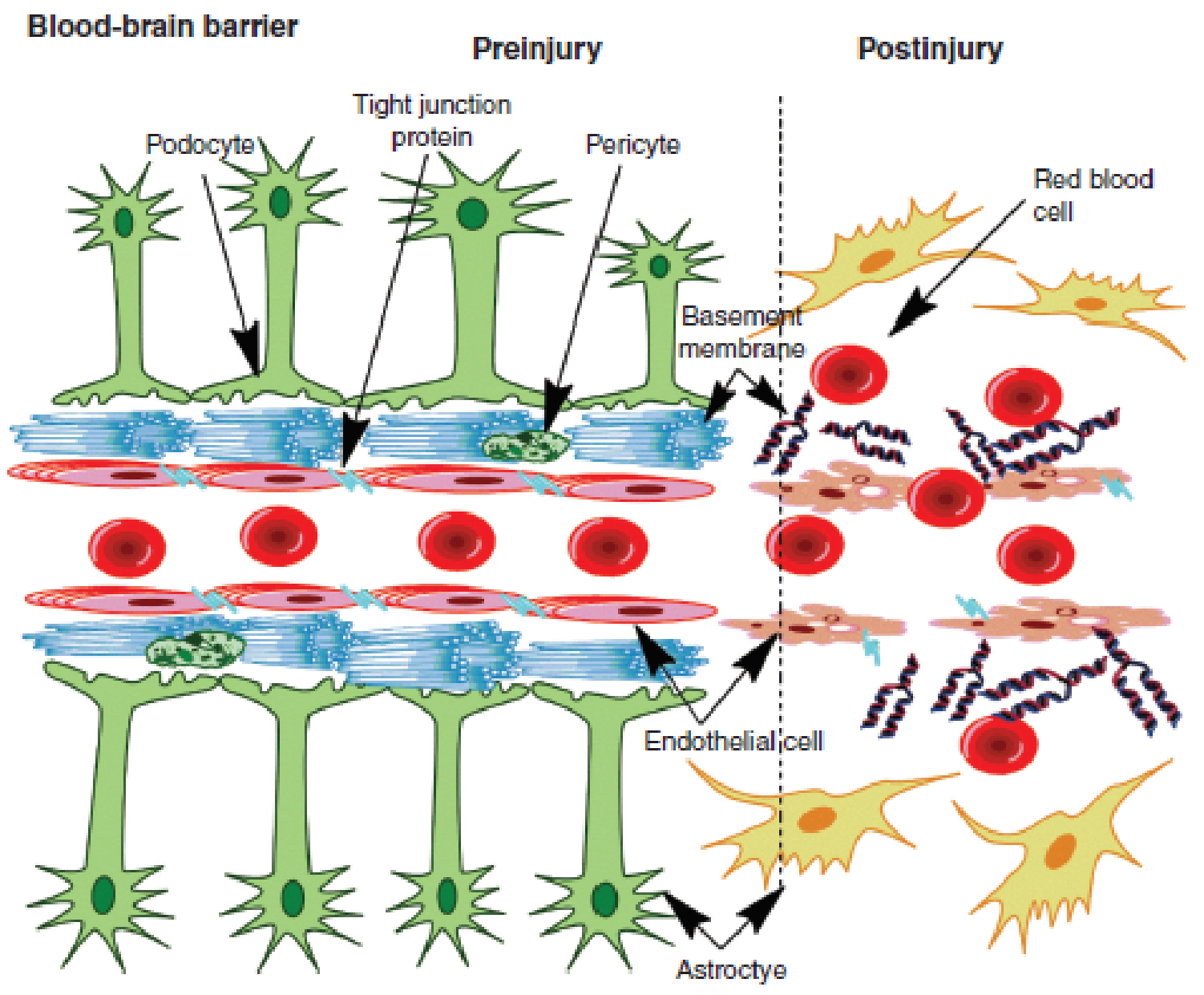

Figure 3 The effects of traumatic brain injury on the blood-brain barrier. TBI can cause disruptions of tight junction proteins connecting endothelial cells. Astrocytes can undergo astrogliosis and the basement membrane can become disrupted. Ultimately, these changes increase the likelihood of red blood cell extravasations. 
neurotoxic proteins into the brain parenchyma (Helmy et al., 2011a; Helmy et al., 2011b).

Prolonged inflammation can eventually contribute to myelin loss (Glushakova et al., 2014).

Omega-3 supplementation in rodents can limit BBB breakdown by inhibiting matrix

metalloproteinase 9 activity (Russell et al., 2014). Targeting estrogen receptors may also be a viable therapeutic target due to decreased BBB permeability following TBI in female rats (Asl et al., 2013).

Tight junctions between endothelial cells maintain vascular integrity, but are often sheared following TBI (Xu et al., 2014a). Blast TBI, in particular, causes a rapid blood pressure spike leading to disruption of the tight junction proteins at the BBB (Yeoh et al., 2013). Alternatively, astrogliosis can also lead to BBB modulation over time (Liu et al., 2014c). Astrocytes support endothelial cell function at the neurovascular unit and direct control of permeability (Molino et al., 2014). Astrocytes and neurons help support the basement membrane surrounding the endothelial cells (Schiera et al., 2003). Podocytes from astrocytes contain vascular endothelial growth factor, which can be released when the BBB is damaged (Saito et al., 2011). The biomarker, pituitary adenylate cyclase activating polypeptide, offers promising diagnostic potential for determining BBB disruption (Bukovics et al., 2014). Currently, digital imaging quantification is the primary method of diagnosis (Biancardi et al., 2014).

\section{Secondary Effects of Traumatic Brain Injury}

Shortly after TBI, biochemical mechanisms assist in repairing cells that survive the primary insults of TBI. On the other hand, those same mechanisms can cause injured cells to die in order to preserve energy for other cells undergoing the energy-dependent repair process. The brain plays a game of checks and balances (using energy as currency) to determine which neuronal cells are worth saving after TBI. Programmed cell death, or apoptosis, is a necessary 
mechanism following neuronal injury and may be beneficial to the CNS. However, the resulting effects from an apoptotic event can cause further damage within the brain through the process of neuroinflammation (Abdul-Muneer et al., 2014).

Even after mild TBI neuronal apoptosis is evident around perivascular regions, indicative of cerebral vascular injury (Abdul-Muneer et al., 2013). Brain damage from TBI was reported to be associated with decreased mitochondrial membrane potential and increased release of cytochrome $\mathrm{C}$ in rodents - both indicative of cellular apoptosis (Wang et al., 2014). Cleavedcaspase-3 and caspase-3 enzyme activity was reportedly increased in TBI animals versus control (Clark et al., 2000). Models of TBI have also shown upregulation of caspase gene expression

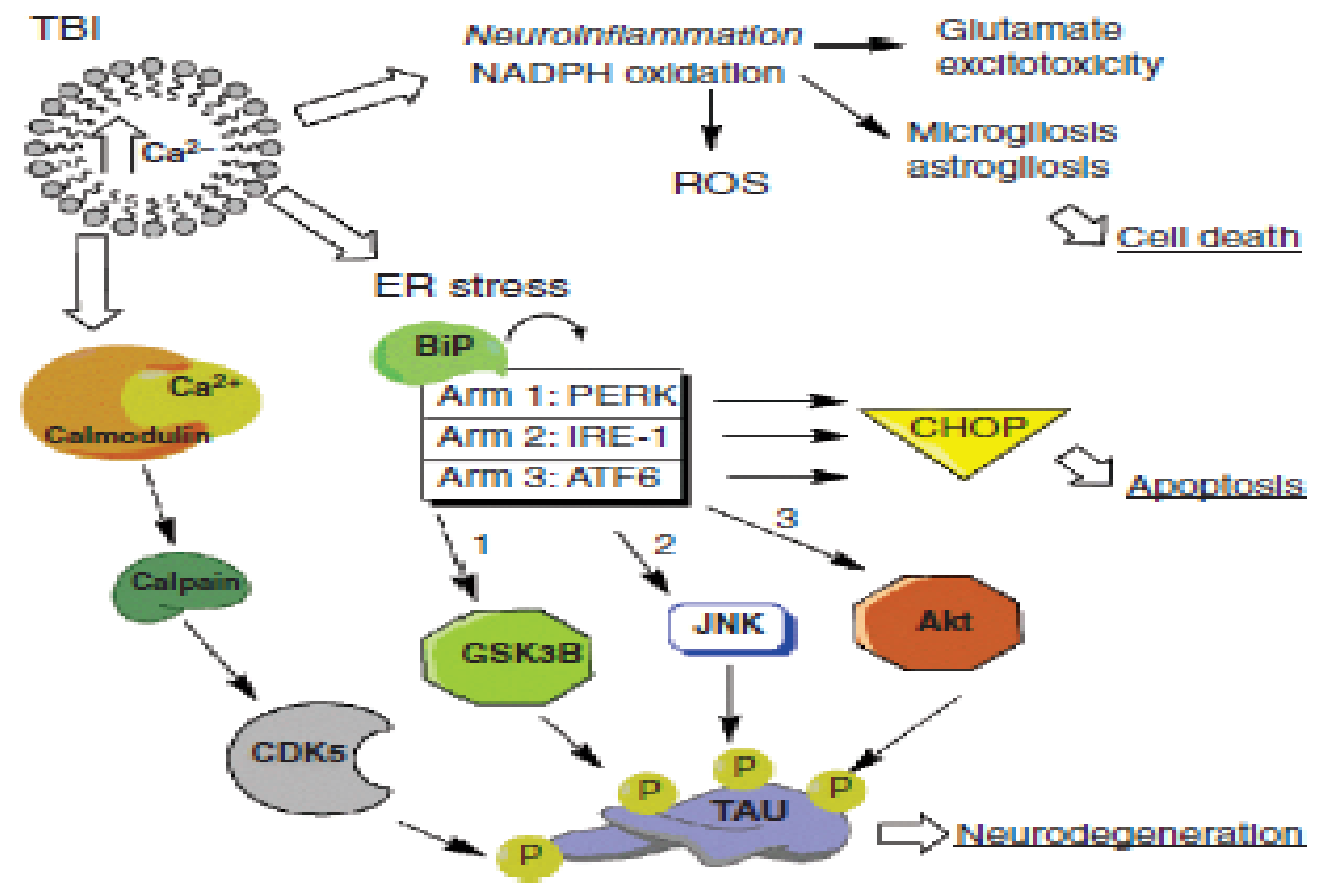

Figure 4 Complex interplay of seoondary mechanisms following traumatic brain injury. Secondary mechanisms of injury have multiple known effects. A few included are cellular necrosis, apoptosis, neurodegeneration, and iauopathy- Timing of activation and pathway connecfions are still being teased out with preclinioal models of TBI. 
(Larner et al., 2004). These results indicate that caspase activity contributes to brain tissue loss, and that caspase inhibition may prove to be an effective treatment strategy for TBI.

The secondary effects of TBI can be attenuated with therapeutic interventions given at the right time following neurotrauma. The series of molecular, neurochemical, cellular, and pathophysiological mechanisms resulting from TBI can be targeted with drug therapy designed to manipulate the signaling mechanisms of various cellular and subcellular processes. The secondary mechanisms of TBI include: neuroinflammation, oxidative stress, endoplasmic reticulum (ER) stress, glutamate excitotoxicity, calpain processing, apoptosis and tau hyperphosphorylation (see Fig. 4). The timing and duration of each event is dependent on the type and severity of the TBI in question; therefore, each treatment strategy could be individualized to the specific type of TBI. The aforementioned secondary mechanisms are described in more detail below.

\section{Endoplasmic Reticulum Stress, Excitotoxicity and Calpain Processing}

TBI occurs over a very short duration, and is often mild in nature, compared to other neural injuries. TBI often goes undetected and chronic deficits often take years to develop (Omalu et al., 2011a; Omalu et al., 2011b). TBI harms neurons by perturbing $\mathrm{Ca}^{2+}$ homeostasis in an energy-independent process. The damage done by TBI makes membranes more permeable to $\mathrm{Ca}^{2+}$ and even to extracellular proteins (Arun et al., 2013). What is known is that neural injury, in particular TBI, causes massive neuronal depolarization and a resulting large influx of $\mathrm{Ca}^{2+}$. Cells which withstand the immediate physical injury from TBI, will have disrupted intracellular $\mathrm{Ca}^{2+}$ levels and oxidative stress (Malhotra and Kaufman, 2007). As described previously, oxidative stress can induce neuroinflammation, which is known to cause neuronal death and is associated with chronic disease pathology (Cernak and Noble-Haeusslein, 2010). 
ER stress has been implicated in a variety of TBI models (Rubovitch et al., 2011a; Farook et al., 2013; Begum et al., 2014). ER stress then triggers the unfolded protein response (UPR) as an endogenous means of cellular repair. TBI-induced $\mathrm{Ca}^{2+}$ perturbations cause proteins to unfold, and when the ER becomes overwhelmed and struggles to re-fold the unfolded proteins, the UPR ensues (Larner et al., 2006). In the short-term, the UPR can promote cell survival through three separate mechanisms: (1) attenuation of global protein translation, (2) upregulation of stress response genes, and (3) degradation of unfolded proteins (Mori, 2000). Apoptosis and neurodegeneration are the end-game consequence if ER stress and the UPR are prolonged (Deniaud et al., 2008; Moreno et al., 2012). The UPR has only recently been addressed by TBI investigators because of its new found link to neurodegeneration (Moreno et al., 2013). The timing and duration of the UPR and how exactly it develops into a neurodegenerative phenotype warrants further investigation.

Another secondary mechanism of TBI also involving calcium disruption is glutamate signaling and the phenomenon known as excitotoxicity. TBI triggers a massive depolarization which promotes excessive glutamate release (Katayama et al., 1990). This disrupted regulation in glutamate signaling plays a role in the pathophysiology of TBI through the initiation of secondary injury cascades (Bullock et al., 1998). High extracellular glutamate promotes high levels of intracellular $\mathrm{Ca}^{2+}$ following TBI (Truettner et al., 2007). Secondary injury cascades initiated by glutamate receptors and the disruption of $\mathrm{Ca}^{2+}$ homeostasis will activate calciumdependent proteases, disrupt energy-dependent processes, and produce oxidative stress (Faden et al., 1989; Yi and Hazell, 2006). Using drug therapy to regulate glutamate release may help to attenuate the secondary effects of TBI exposure. 
Loss of microvascular integrity and BBB compromise are often related to brain injuries involving $\mathrm{Ca}^{2+}$ perturbations. In brain trauma models acute and sustained activation of the calpain family of proteases has been implicated in neuronal death and TBI (Liu et al., 2014a). Calpains are acutely activated following TBI within the injured hippocampus and frontal cortex (Ringger et al., 2004). Calpain activation has been observed in degenerating dendrites and atrophic neurons after TBI, providing evidence that calpain activation may participate in neuronal loss after neurotrauma (Huh et al., 2006). Activation of $\mathrm{Ca}^{2+}$-dependent proteases, such as calpain, is a predicted outcome of membrane depolarization and loss of $\mathrm{Ca}^{2+}$ homeostasis. Its regulation of cytoskeletal dynamics contributes to plasticity (Greer et al., 2013) and is consistent with injury deficits in axonal architecture and disruption of synaptic plasticity (Dapul et al., 2013). Animals exposed to TBI can exhibit damaged axonal hillocks, suggesting detrimental changes to their synaptic function (Baalman et al., 2013). Moreover, calpain-dependent processes in TBI models have been suggested to attenuate overall electrophysiological responses (Park et al., 2013) and invoke more neuronal death (Schafer et al., 2009).

\section{Chronic Effects of Traumatic Brain Injury}

Alteration of cerebral blood flow has been linked to delayed neuronal death in the contusion penumbra (Engel et al., 2008). Hemorrhagic shock causes immediate reduction in mean arterial pressure with continued apoptosis over time due to perpetual lack of perfusion (Dennis et al., 2009). At extended time points TBI continues to cause disruption in the autoregulation of cerebral vasculature (Pop and Badaut, 2011). A primary mechanism is temporal changes in V1a vasopressin receptors (Szmydynger-Chodobska et al., 2004). Vasopressin receptor stimulation contributes to cerebral edema that leads to increased intracellular uptake of $\mathrm{H}_{2} \mathrm{O}$ from the blood (Marmarou et al., 2014). The disruption in vascular 
supply can contribute to perivascular nerve damage (Ueda et al., 2006). In addition, systemic hypotension can contribute to chronic neurodegeneration (Long et al., 2009). Persistent activation of calpain proteases is another mechanism by which persistent hypotension leads to neuronal pathology (Newcomb et al., 1999). Calpains cleave the contractile components of vascular smooth muscles inhibiting their ability to contract (Neuhof and Neuhof, 2014).

Not surprisingly, cerebral hypotension has been associated with neurodegenerative diseases such as Alzheimer's disease and Parkinson's disease (Le Heron et al., 2014). A genetic mutation in Neurogenic locus notch homolog protein 3 increases the risk for small vessel disease following TBI and has been linked as well to Alzheimer's disease (Marchesi, 2014). Interestingly, microvasular defects may be linked to Alzheimer's disease due to the increased incidence of neurological disease in TBI patients (Dennis et al., 2009). Preventative treatment approaches are limited, but a few experimental techniques appear promising. Closely regulated hypothermia therapy can prevent chronic microvessel changes and contustion expansion (Zhong et al., 2009), while compressing the internal jugular vein has also been shown to mitigate axonal injury and vascular disruption (Smith et al., 2012; Turner et al., 2012b). Because TBI cannot be predicted a post-injury therapy that mitigates the detrimental chronic effects is therefore needed. Tau protein hyperphosphorylation and amyloid beta oligomers are well-known markers of neurodegenerative disease. TBI has been shown to hyperphosphorylate tau protein (Goldstein et al., 2012), and produce amyloid beta oligomers (Smith et al., 2003), through mechanisms that are not completely understood. These neurodegenerative factors are activated immediately following TBI and can advance profoundly over time (see Fig. 5). The cellular repair mechanisms associated with these factors are more drawn out, and may contribute to neurodegenerative disease progression and brain matter loss (Hoozemans et al., 2007; Scheper et 
al., 2011). Cortical tissue loss and white matter atrophy resulting from TBI are associated with cognitive deficits (Huh et al., 2008). Cognitive deficits in rodents have been observed at chronic time points following TBI (Petraglia et al., 2014c), indicating neurodegenerative disease progression in rodent models of TBI. How the primary effects of TBI manifest into chronic disease states is an area of ongoing investigation. The brains of professional athletes and military veterans are currently being examined with new imaging modalities to discover underlying causes behind neuropsychiatric symptoms such as post-traumatic stress disorder, chronic traumatic encephalopathy, and Alzheimer's disease (Small et al., 2013). Moving forward, TBI research needs to emphasize vascular effects, glial responses and resulting neurodegeneration.

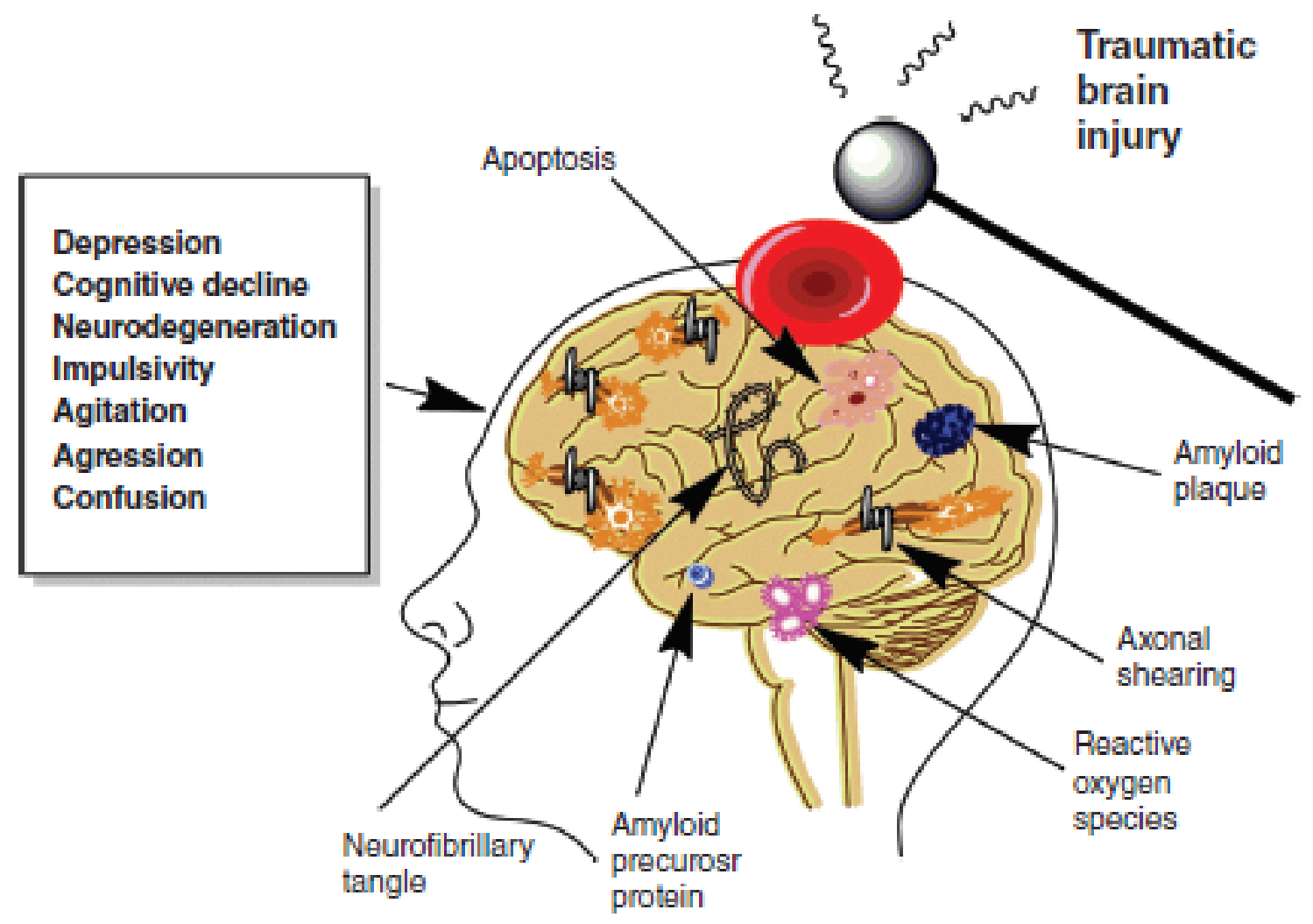

Figure 5 The chronic effects of traumatic brain injury. Some individuals experiencing TBI are more susceptible to chronic effects than others. Environmental and genetic factors play a role. Pathologic changes that may develop include neurofibrillary tangles, axonal shearing, and amyloid plaques. Neuropsychiatric symptoms may also develop such as depression, impulsivity, cognitive decline, and confusion. These chronic effects following TBI remains a topic of growing importance receiving renewed research focus and funding. 


\section{Future Directions}

Primary and secondary mechanisms of TBI are undergoing continued investigation. What remain to be determined are the tertiary mechanisms that mark the transition from acute injury to chronic neurodegeneration. Studying the broader role of cerebrovascular changes and how these changes interact with neurons and glia must be carefully considered in future investigations. Chronic mechanisms might very well deal with the vasoregulation of cerebral vessels and how microvessels respond to inflammatory markers. In addition, the theory of 'inflamm-aging' is likely to take precedence, because aged neurons and glia respond differently to injury-induced stimuli. How these aged cells interact with concurrently aged and previously injured vascular system has not been fully elucidated. Chronic disease states are subject to the effects of both agerelated comorbidities and aging itself.

Future studies must incorporate a broader approach utilizing more representative models. Animal models of TBI have been developed, but therapeutics have not yet translated successfully to the clinic. Therefore, an increasing need exists for the development of more clinically relevant TBI models, and the use of advanced MRI and PET imaging to map the dynamics of brain injury responses. This will enable us to devise more effective therapeutic interventions for the amenable secondary effects. Further studies utilizing BBB compromise following TBI could be advantageous for pharmaceutical researchers attempting to alter brain mechanisms. In addition, the balance between protein phosphatases vs. kinases will need to be heavily investigated. Marking the transition to amyloidopathy and/or tauopathy will provide important insight into effective treatment windows. More importantly, understanding chronic mechanisms of injury will allow a more tailored and individualized approach to patient care. 


\section{Table 1.1: Forms of Traumatic Brain Injury}

\begin{tabular}{|c|c|c|c|}
\hline Type & Description & Animal model & References \\
\hline $\begin{array}{l}\text { Blast traumatic } \\
\text { brain injury }\end{array}$ & $\begin{array}{l}\text { Blast energy generated from an explosive device } \\
\text { contacts the skull and causes acceleration/ } \\
\text { deceleration injury }\end{array}$ & $\begin{array}{l}\text { Blast model with short } \\
\text { driver section for clinical } \\
\text { relevance }\end{array}$ & $\begin{array}{l}\text { Alford et al., 2011, Arun et al., } \\
2013 \text {, Cho et al., 2013, DeWitt } \\
\text { et al., 2009, Turner et al., } 2013\end{array}$ \\
\hline $\begin{array}{l}\text { Closed head } \\
\text { impact }\end{array}$ & $\begin{array}{l}\text { Blunt object strikes the skull without fracturing the } \\
\text { bone }\end{array}$ & Impact-acceleration model & Li et al., 2013, Turner et al., 2012 \\
\hline $\begin{array}{l}\text { Concussion and } \\
\text { subconcussion }\end{array}$ & $\begin{array}{l}\text { A temporary state of shock due to a violent blow } \\
\text { to the head }\end{array}$ & $\begin{array}{l}\text { Controlled closed-head } \\
\text { impact with helmet }\end{array}$ & $\begin{array}{l}\text { Angoo-Perez et al., 2014, Bailes } \\
\text { et al., 2013, Petraglia et al., } 2014\end{array}$ \\
\hline Contusion & $\begin{array}{l}\text { An area of injured brain where blood capillaries } \\
\text { have been ruptured }\end{array}$ & Fluid Percussion model & $\begin{array}{l}\text { Greer et al., 2013, Truettner et al., } \\
2007\end{array}$ \\
\hline Falls & $\begin{array}{l}\text { Rapid impact between ground or object with } \\
\text { skull due to effects of gravity }\end{array}$ & Weight drop TBI model & $\begin{array}{l}\text { Albert-Weissenberger and Siren, } \\
2010 \text {, Asl et al., } 2013\end{array}$ \\
\hline $\begin{array}{l}\text { Penetrating } \\
\text { injury }\end{array}$ & $\begin{array}{l}\text { Fragment from shrapnel or blunt object that } \\
\text { fractures skull and causes protrusion into brain } \\
\text { parenchyma }\end{array}$ & $\begin{array}{l}\text { Controlled cortical impact } \\
\text { with craniectomy }\end{array}$ & $\begin{array}{l}\text { Begum et al., 2014, Clark et al., } \\
1997\end{array}$ \\
\hline $\begin{array}{l}\text { Shaken baby } \\
\text { syndrome }\end{array}$ & $\begin{array}{l}\text { Abusive head trauma due to rapid acceleration } \\
\text { and deceleration of skull when shaken }\end{array}$ & $\begin{array}{l}\text { Controlled cortical impact } \\
\text { with intact skull }\end{array}$ & $\begin{array}{l}\text { Dileonardi et al., } 2009 \text {, Foster et al., } \\
2014 \text {, Larner et al., } 2004\end{array}$ \\
\hline $\begin{array}{l}\text { Spinal cord } \\
\text { injury }\end{array}$ & $\begin{array}{l}\text { Injury to any part of the spinal cord that leads to } \\
\text { inflammation and secondary effects on the CNS }\end{array}$ & Spinal impact model & $\begin{array}{l}\text { Faden et al., 1989, Roth et al., } \\
2014 \text {, Xiong et al., } 2013\end{array}$ \\
\hline
\end{tabular}




\section{Table 1.2: Neurotoxic Events in Traumatic Brain Injury}

\begin{tabular}{|c|c|c|}
\hline Primary & Acute phase physiology & References \\
\hline $\begin{array}{l}\text { Intracranial pressure spike } \\
\text { hemorrhage }\end{array}$ & $\begin{array}{l}\text { Vascular pressure spike bursts brain microvessels; brain hemorrhage } \\
\text { releases blood-borne toxins and iron into parenchyma }\end{array}$ & $\begin{array}{l}\text { Arun et al., 2013, } \\
\text { Abdul-Muneer et al., } 2014\end{array}$ \\
\hline Axonal shearing & $\begin{array}{l}\text { External forces physically tear axons; impairs axonal transport and } \\
\text { synaptic function }\end{array}$ & $\begin{array}{l}\text { Raghupathi et al., 2002, } \\
\text { Dileonardi et al., } 2009\end{array}$ \\
\hline Vasospasm ischemia & $\begin{array}{l}\text { Blood flow disrupted via uneven constriction and dilation in brain } \\
\text { vasculature }\end{array}$ & $\begin{array}{l}\text { Izzy et al., 2014, } \\
\text { Foster et al., } 2014\end{array}$ \\
\hline Secondary & Subacute phase physiology & \\
\hline $\begin{array}{l}\text { Reactive oxygen species } \\
\text { Oxidative stress }\end{array}$ & $\begin{array}{l}\text { Mitochondria } \mathrm{Ca}^{2+} \text { imbalance, mixed with energy failure and NADPH } \\
\text { oxidation, triggers oxidative stress and neuronal cell death }\end{array}$ & $\begin{array}{l}\text { Deniaud et al., } 2008 \text {, } \\
\text { Cho et al., } 2013\end{array}$ \\
\hline Neuroinflammation & $\begin{array}{l}\text { Cytokine release from glial cells promotes cytotoxic effects through } \\
\text { macrophage differentiation }\end{array}$ & $\begin{array}{l}\text { Szmydynger-Chodobska et al., } \\
2004 \text {, Roth et al., } 2014\end{array}$ \\
\hline Blood-brain-barrier disruption & $\begin{array}{l}\text { Release of blood toxins into brain parenchyma promoting inflammation } \\
\text { and neuronal death }\end{array}$ & $\begin{array}{l}\text { Abdul-Muneer et al., 2013, } \\
\text { Alves } 2014\end{array}$ \\
\hline Endoplasmic reticulum stress & $\begin{array}{l}\text { Overabundance of unfolded proteins and intracellular } \mathrm{Ca}^{2+} \text { triggers the } \\
\text { unfolded protein response }\end{array}$ & $\begin{array}{l}\text { Truttner et al., } 2007 \text {, Rubovitch } \\
\text { et al., 2011, Larner et al., } 2004\end{array}$ \\
\hline Glutamate excitotoxicity & Reduced glutamate uptake activates NMDA receptors & $\begin{array}{l}\text { Bullock et al., } 1998, \\
\text { Katayama et al., } 1990\end{array}$ \\
\hline Protease activation & $\begin{array}{l}\text { Intracellular } \mathrm{Ca}^{2+} \text { binds to calmodulin forming calpain kinase; calpain } \\
\text { activation cleaves p } 35 \text { into toxic p } 25\end{array}$ & $\begin{array}{l}\text { Ringger et al., } 2004, \\
\text { Huh et al., } 2006\end{array}$ \\
\hline Apoptosis & $\begin{array}{l}\text { Intrinsic pathway activation both in mitochondria and Endoplasmic } \\
\text { reticulum; } \mathrm{Ca}^{2+} \text { dependent processes }\end{array}$ & $\begin{array}{l}\text { Clark et al., } 2000 \text {, } \\
\text { Nakagawa et al., } 2000\end{array}$ \\
\hline Chronic & Chronic phase physiology & \\
\hline Tau phosphorylation & $\begin{array}{l}\text { Tau kinase/phosphatase imbalance leads to hyperphosphorylation of } \\
\text { tau }\end{array}$ & $\begin{array}{l}\text { Goldstein et al., 2012, } \\
\text { Begum et al., } 2014\end{array}$ \\
\hline Amyloid beta & $\begin{array}{l}\text { Increased amyloid precursor protein promotes the toxic oligomerization } \\
\text { of amyloid beta }\end{array}$ & $\begin{array}{l}\text { Smith et al., } 2003 \text {, } \\
\text { O'Connor et al., } 2008\end{array}$ \\
\hline $\begin{array}{l}\text { Neurodegeneration } \\
\text { Cognitive deficits }\end{array}$ & $\begin{array}{l}\text { Tau hyperphosphorylation forms neurofibrillary tangles near perivascular } \\
\text { regions; promoting neurodegenerative disease and cognitive decline }\end{array}$ & $\begin{array}{l}\text { Mckee et al., 2014, } \\
\text { Bailes et al., } 2013\end{array}$ \\
\hline
\end{tabular}




\section{OBJECTIVES}

\section{Study I: Validate neural injury in the rat model of traumatic brain injury over time}

Blast-induced traumatic brain injury represents a leading cause of injury in modern warfare with injury pathogenesis poorly understood. Preclinical models of blast injury remain poorly standardized across laboratories and the clinical relevance unclear based upon pulmonary injury scaling laws. Models capable of high peak overpressures with short durations may better replicate clinical exposure when scaling principles are considered. In this work we demonstrate a tabletop shock tube model capable of high peak overpressures of short duration. By varying the thickness of the polyester membrane, peak overpressure could be controlled. Blast exposure was shown to decrease total activity and histology revealed markers of neuronal degeneration, increased glial activation, and Blood-brain barrier disruption. This work demonstrates one of the first examples of blast-induced brain injury in the rodent when exposed to a blast wave scaled from human exposure based on scaling principles derived from pulmonary injury lethality curves.

\section{Study II: Characterize traumatic brain injury in the rat by investigating primary injury effects, secondary injury cascades, neurophysiology and neurobehavioral dysfunction}

Traumatic brain injury resulting from blast exposure is the most common injury of military combatants. Animal modeling of blast-induced traumatic brain injury has demonstrated severe histopathological effects accompanied by loss of long-term synaptic plasticity and cognitive 
performance due to tissue damage. Here we use a scaled blast model that was specifically developed to assess the neuropathological, neurophysiological, and biochemical effects of exposure to blast forces not severe enough to cause overt physiological deficits or behavioral changes. We find that mild blast exposure induces no observable macroscopic histological effects, but instead causes microvascular injury, axonal damage, and neuroinflammation in various brain regions including hippocampus. Hippocampal deficits in short term plasticity and synaptic excitability were observed after mild blast exposure. Biochemical analysis revealed that mild blast exposure invokes markers of excitotoxicity and neuronal injury in hippocampus. Thus mild blast exposure causes subtle but deleterious neurological effects in the absence of major behavioral deficits, suggesting that subclinical exposures to blast forces may initiate neuropathology.

\section{Study III: Investigate the effects of endoplasmic reticulum stress modulation on cellular fate and impulsive-like behavior in rats exposed to traumatic brain injury}

Neuronal injury following blast-induced traumatic brain injury increases the risk for neuropsychiatric disorders, yet the pathophysiology remains poorly understood. Blood-brainbarrier disruption, endoplasmic reticulum stress, and apoptosis have all been implicated in neurotrauma. Microvessel compromise is a primary effect of neurotrauma and is postulated to cause subcellular secondary effects. What remains unclear is how these secondary effects progress to personality disorders in humans exposed to head trauma. To investigate this we exposed male rats to a clinically relevant traumatic brain injury model we have recently developed. The study examined initial Blood-brain barrier disruption, endoplasmic reticulum stress mechanisms, 
apoptosis and impulsive-like behavior. The endoplasmic reticulum stress inhibitor, salubrinal, was administered (1mg/kg i.p.) to investigate its' effects on neuronal injury and impulsive-like behavior associated with neurotrauma. Results would provide evidence that neurotrauma involves endoplasmic reticulum stress and suggest a promising molecular target for the attenuation of neuronal injury and amelioration of neuropsychiatric symptoms after neurotrauma.

\section{Study IV: Investigate the effects of endoplasmic reticulum stress modulation on neuroinflammation and risk-taking behavior in rats exposed to repetitive traumatic brain injury}

TBI is the leading cause of trauma related morbidity in the developed world. TBI has been shown to trigger secondary injury cascades including endoplasmic reticulum stress, oxidative stress, and neuroinflammation. The link between secondary injury cascades and behavioral outcome following TBI is poorly understood and warrants further investigation. Using our validated rodent blast TBI model, we examined the secondary injury cascades following single injury and how these effects may contribute to risk-taking behavior after repetitive TBI. We targeted these pathways with the cellular stress modulator, salubrinal, and investigated its role in decreasing neuroinflammation and risk-taking behavior after blast injury. Overall, these results would suggest a novel approach to treat neuroinflammation and neuropsychiatric symptoms following neurotrauma.

\section{Study VI: Model chronic traumatic encephalopathy in multiple models of traumatic brain injury}


Chronic neurodegeneration following a history of neurotrauma is frequently associated with neuropsychiatric and cognitive symptoms. In order to enhance understanding about the underlying pathophysiology linking neurotrauma to neurodegeneration, a multi-model preclinical approach must be established to account for the different injury paradigms and pathophysiologic mechanisms. We investigated the development of tau pathology and behavioral changes using a multi-model and multi-institutional approach, comparing the pre-clinical results to tauopathy patterns seen in post-mortem human samples from athletes diagnosed with chronic traumatic encephalopathy (CTE). We utilized a scaled and validated blast-induced traumatic brain injury model in rats and a modified pneumatic closed-head impact model in mice. Tau hyperphosphorylation was evaluated by western blot and immunohistochemistry. Elevated plus maze and Morris water maze were employed to measure impulsive-like behavior and cognitive deficits respectively. This study provides a multi-model example of replicating tau and behavioral changes in animals and provides a foundation for future investigation of CTE disease pathophysiology and therapeutic development. 


\section{RATIONALE}

Chronic traumatic encephalopathy (CTE) is a serious neuropsychological disease identified in a growing number of soldiers and athletes exposed to repetitive traumatic brain injuries (TBIs). This progressive neurodegenerative disease is distinguished neuropathologically by neurofibrillary tau tangles with a predilection for perivascular and subcortical areas near reactive astrocytes. The disease is currently diagnosed post-mortem by pathologic identification of the neurofibrillary tangles. There is a need for pre-mortem diagnosis in an effort to understand disease pathophysiology and to develop targeted treatments. In order to accomplish this objective, it is necessary to discover the mechanistic link between acute neurotrauma and the development of chronic neurodegenerative and neuropsychiatric disorders such as CTE. The role of endoplasmic reticulum (ER) stress is important to investigate since protein kinase-like ER kinase (PERK), a key marker of ER stress, is co-localized with hyperphosphorylated tau in pretangle neurons. The long-term goal of this work is to understand how ER stress modulates and/or causes the neurofibrillary tau tangles seen in CTE. The central hypothesis is that ER stress contributes to the neuropathological findings of CTE, and that modulation of ER stress will improve outcomes. The significance of this proposal is that by examining and manipulating ER stress, a disease mechanism associated with tau hyperphosphorylation, we will aid in finding diagnostic and therapeutic treatment options which will have a substantial impact on overall understanding of acute brain injuries as well as chronic neurodegenerative diseases.

By understanding the role that ER stress plays in the progression of CTE, it will facilitate the development of urgently needed diagnostics and therapeutics. New imaging modalities and biomarkers specifically focused on the identification of ER stress proteins will potentially prove to be an invaluable resource for diagnosing CTE following bTBI. In addition, ER stress 
modification using a drug such as salubrinal will not only provide an effective treatment option but more importantly a preventative solution to mitigate CTE progression. The far reaching implications of this proposed research will improve health outcomes and disease management not only for CTE but also for related neurodegenerative diseases.Regardless of the time course of activation mediated by the different arms of the ER stress pathway, the valuable information obtained about the transition phase between cell survival and cell death will provide a strong line of evidence for exactly when the process of neurodegeneration begins following blast injury. Understanding the role of ER stress in bTBI will improve our ability to locate pharmacologic targets to treat and ideally prevent CTE.

TBI has profound and measurable effects on cerebral vasculature. Due to the variable expression of TBI in the hospital setting, as characterized by the Glasgow Coma Scale and advanced imaging, individual treatment approaches are a necessity. Understanding complication of TBI such as subarachnoid hemorrhage, vasospasm, and ischemia are necessary in appropriate patient management. Mechanism of injury such as acceleration/deceleration, axonal damage, and vascular disruption are also important to keep in mind. The most promising targets include secondary injury cascades such as oxidative stress, neuroinflammation, and ER stress. Future approaches may target chronic mechanisms involved in the development of neurodegenerative disease. Better pre-clinical models are necessary to map more complex systems such as glutamate toxicity and calpain mediated cell death. Moving forward, treatment of secondary mechanisms affecting vascular dynamics is critical both acutely and chronically. Consideration of age and comorbidities remains a constant factor in any form of neuronal injury. Neurodegeneration and cellular apoptosis are common following TBI, but improved management acutely can prevent the common sequelae of symptoms. In order for successful 
therapeutic options to translate to the clinic, TBI research needs to move away from the common neurocentric approach and change focus towards vascular effects, glial responses and neurodegeneration.

In summary, ER stress, and the UPR, is activated following TBI. The primary effects of TBI can produce excess ROS, increased intracellular $\mathrm{Ca}^{2+}$ levels, and induce inflammation; however, the timing, severity, and duration all vary from model to model. It is difficult to prevent the immediate effects of TBI, but we can attempt to mitigate the secondary effects that follow. Preventative therapies, mostly pharmacological agents, have been employed in pre-clinical models of neural injury, but nothing has yet to successfully translate to the clinic. The approach has been to mediate apoptosis and reduce neurodegeneration. However, the underlying link between ER stress, apoptosis and neurodegeneration is not yet fully understood. Research is now being focused on the most effective treatment strategies to suppress chronic effects resulting from neural injury. Of particular importance is transferring preclinical success to effective treatment options for human care. Evidence suggests that combining therapeutic options that target multiple injury components at the ideal time window will be the most effective strategy going forward.

Our expectations are that we will determine the role of ER stress in CTE development and progression. Furthermore, we will show that appropriate manipulation of the ER stress pathway will improve outcomes in preclinical study, thus justifying the need for further studies, including those in a clinical population. The significance of this proposal is that by examining and manipulating the mechanistic process of this disease we will aid in finding diagnostic and therapeutic treatment options, which will have a substantial impact on overall understanding of acute brain injuries as well as chronic neurodegenerative diseases. 


\section{Chapter Two}

Modeling clinically relevant blast parameters based on scaling principles produces functional \& histological deficits in rats

This work is published in Exp Neurol. 2013 Oct;248:520-9.

doi: 10.1016/j.expneurol.2013.07.008. Epub 2013 Jul 20. PMID: 23876514

Turner RC, Naser ZJ, Logsdon AF, DiPasquale KH, Jackson GJ, Robson MJ, Gettens RT, Matsumoto RR, Huber JD, Rosen CL. 


\section{ABSTRACT}

Blast-induced traumatic brain injury represents a leading cause of injury in modern warfare with injury pathogenesis poorly understood. Preclinical models of blast injury remain poorly standardized across laboratories and the clinical relevance unclear based upon pulmonary injury scaling laws. Models capable of high peak overpressures with short durations may better replicate clinical exposure when scaling principles are considered. In this work we demonstrate a tabletop shock tube model capable of high peak overpressures of short duration. By varying the thickness of the polyester membrane, peak overpressure can be controlled. We used membranes with a thickness of $0.003,0.005,0.007$, and 0.010 inches to generate peak reflected overpressures of 31.47, 50.72, 72.05, and 90.10 PSI, respectively. Blast exposure was shown to decrease total activity and produce neural degeneration as indicated by fluoro-jade B staining. Similarly, blast exposure resulted in increased glial activation as indicated by an increase in the number of glial fibrillary acidic protein expressing astrocytes compared to control within the corpus calllosum, the region of greatest apparent injury following blast exposure. Similar findings were observed with regards to activated microglia, some of which displayed phagocytic-like morphology within the corpus callosum following blast exposure, particularly with higher peak overpressures. Furthermore, hematoxylin and eosin staining showed the presence of red blood cells within the parenchyma and red, swollen neurons following blast injury. Exposure to blast with 90.10 PSI peak reflected overpressure resulted in immediate mortality associated with extensive intracranial bleeding. This work demonstrates one of the first examples of blast-induced brain injury in the rodent when exposed to a blast wave scaled from human exposure based on scaling principles derived from pulmonary injury lethality curves. 


\section{INTRODUCTION}

Blast-induced traumatic brain injury has been described as the hallmark injury of modern warfare (Elder and Cristian, 2009; Nakagawa et al., 2011) with 25,000 members of the armed services (U.S. and Coalition forces) injured or killed by explosive devices in Iraq and Afghanistan conflicts in the past decade (Champion et al., 2009). The prevalence of blast exposure is associated with an immense financial and societal burden with estimates of $13-22 \%$ of combat veterans having sustained a traumatic brain injury (TBI) during time in service (Schneiderman et al., 2008; Terrio et al., 2009; Panzer et al., 2012). Consequently, there is a clear clinical need for increased understanding of blast injury pathogenesis and the development of improved therapeutics for the treatment, or prevention, of blast-induced brain injury. Increasing efforts to study blast injury has resulted in the propagation of numerous preclinical models of blast injury with the shock tube representing the most widely used model type. Despite the extensive use of shock tube models, model parameters such as size of the driver and driven sections, membrane material and thickness, and gas used seem to vary across laboratories. As such, the shock waves produced vary significantly in peak overpressure and duration.

Recent work by Panzer and colleagues has identified potential discrepancies between many preclinical models and real-world recorded blast parameters with models often exposing small rodents to long duration blasts (Panzer et al., 2012). Assuming the same scaling principles apply as those discovered with regards to pulmonary blast injury, many current rodent models, in which overpressure exposure durations frequently exceed $4 \mathrm{~ms}$ and in some cases as much as $10 \mathrm{~ms}$ or more, may be subjecting animals to the equivalent of a human exposed to detonation of $27,000-\mathrm{kg}$ of TNT from over $70 \mathrm{~m}$ away (Panzer et al., 2012). In other words, many preclinical models may be exposing animals to the equivalent of long-duration nuclear blasts in humans rather than the 
more common high explosive blasts (Bass et al., 2012). Therefore, a preclinical blast model capable of delivering high peak overpressures over short durations may be of value, particularly should similar scaling laws exist in brain, as is the case in pulmonary-based work.

In this work we demonstrate the ability to model blast injury with clinically relevant peak overpressure and duration based on scaling laws from humans to rodents with a novel, tabletop shock tube model. Furthermore, we show the ability to model blast injury of varying severity with different PET membranes that result in reflected peak overpressures ranging from 31-90 PSI while consistently being of short duration $(<3 \mathrm{~ms})$. Animals exposed to blast-induced traumatic brain injury across this range of pressures exhibit alterations in total activity as well as clear histological changes as determined utilizing common markers of neural injury.

\section{METHODS}

\section{Animals}

All procedures involving live animals were approved by the Institutional Animal Care and Use Committee of West Virginia University and were performed according to the principles of the Guide for the Care and Use of Laboratory Animals. This worked used thirty 350 gram male animals acquired from Hilltop Lab Animals (Hilltop Lab Animals, Inc.). Animals were acclimated for 1 week prior to experimental use and were housed under 12-hour light/12-hour dark conditions with food and water available ad libitum.

\section{Design of Blast Model}

A 4-piece, machined aluminum shock tube apparatus was constructed and driven using compressed nitrogen gas. The driver and driven sections were separated by clear mylar membranes (Ridout Plastics Co.) of varying thickness (0.003"-0.010”) to achieve a range of peak 
overpressure exposures. A tapered design of the driver section (Figure 1) was included in an effort to minimize wave reflection and alterations of the blast wave by the expansion wave. This feature is similar in nature to blast tubes utilizing explosives in which the explosive is often detonated in a conical or parabolic-shaped driver section. The driver section was kept short in length in an effort to reduce the amount of gas required for membrane rupture and subsequent blast duration. The overpressure duration and impulse are reduced as described previously due to the shortened driver section (Bass et al., 2012). For additional details concerning design of the blast model, please see Supplemental Information.

\section{Blast Waveform Acquisition \& Analysis}

Shock wave pressures were detected using piezoelectric sensors (PCB Piezotronics) that were placed in both reflected and incident positions at the exit of the shock tube (Figure 1). Data was acquired and processed using the National Instruments input module (NI 9223) connected to the National Instruments receiver USB chassis (CDAQ-9171). Signals were recorded using a custom-designed Labview 12.0 program and the NI data acquisition system (National Instrument) with a sampling rate of $500 \mathrm{kHz}$ as described previously (Chavko et al., 2011; Ahlers et al., 2012).

\section{Blast Exposure}

Prior to blast exposure, animals were anesthetized by intraperitoneal injection of ketamine (90 mg/kg; Webster Veterinary) and xylazine (5 mg/kg; Webster Veterinary). Animals were oriented with the long axis of the animal perpendicular to the blast front. In other words, the blast was delivered side-on to the head only as the thorax and abdomen were protected using rigid shielding, creating an experimental setup (Figure 1) similar to that used by Shridharani and colleagues (Shridharani et al., 2012). With the animal placed directly at the exit of the tube, the effect of expansion waves on the planar conditions of the blast front is minimized as described by 
Bass and colleagues (Bass et al., 2012). Animals ( $\mathrm{n}=38$ ) were divided into the following groups: control $(n=10), 0.003$ membrane blast exposure $(n=6), 0.005$ membrane blast exposure $(n=10)$, 0.007 membrane blast exposure $(n=9)$, and 0.010 membrane blast exposure $(n=3)$. Immediately following blast exposure, animals were returned to a holding cage equipped with a homeothermic heating blanket equipped with a rectal thermometer to maintain body temperature at $37^{\circ} \mathrm{C}$. Once basic reflexes were restored, animals were returned to the home cage.

\section{Total Activity Measurements}

Animal activity was measured using an automated activity monitoring system (San Diego Instruments). Rats were given 1 hour to acclimate to the testing room prior to initiation of testing. Each testing chamber consisted of a $16 \times 16$ photobeam array surrounding a Plexiglass chamber to record lateral movements. A separate 8 photobeam array to detect rearing activity was located above the $16 \times 16$ array. Activity was quantified over a 30 minute period 24 hours post-blast exposure with total activity, ambulatory, and fine activity reported based on default settings of the data acquisition software (PAS, San Diego Instruments).

\section{Histological Preparations}

At 72 hours post-blast exposure, a total of 24 animals ( 6 control and 6 from each blast exposure group) were anesthetized as described above and perfused transcardially with cold $0.9 \%$ saline followed by $10 \%$ formalin for a total of ten minutes. The brain was then extracted and placed into fresh $10 \%$ formalin for a minimum of 24 hours. After 24 hours of fixation, the brain was blocked into sections and paraffin embedded as previous described (Turner et al., 2012c). Briefly, tissues were processed using the Tissue-Tek VIP 5 automatic tissue processor (Sakura Finatek) and embedded in paraffin using the Tissue-Tek TEC 5 embedding system (Sakura Finatek). Tissues were sliced using a Leica RM2235 microtome (Leica Microsystems) and slices 
mounted on slides for staining. Standard protocols were utilized for staining with hematoxylin and eosin (H\&E), fluorojade-B (Millipore), which was used as a marker of neural degeneration, glial fibrillary acidic protein (Dako), which was used as a marker of reactive astrocytes, and ionized calcium binding adapter molecule (Wako), which was used as a marker of activated microglia. Images were acquired from the S1BF region of the cortex, corpus callosum, striatum, hippocampus, and cerebellum. Imaging was performed using a Zeiss Axio Imager 2 for all brightfield and fluorescent images shown.

\section{Histological Quantification}

Stereology and the optical fractionator technique were used to quantify histological results as previously described. (Schmitz and Hof, 2005; Smith et al., 2012; Turner et al., 2012b) Briefly, a region of interest encompassing the corpus callosum was drawn at low power using an Olympus AX70 microscope and StereoInvestigator software. The software then selected random $75-\mu \mathrm{m}$ counting frames with a depth of $6 \mu \mathrm{m}$, and the object of interest marked by an observed blinded to treatment. The volume of the region of interest previously identified is then determined by the software and the number of cells marked by the observer returned.

\section{Quantitative real-time PCR}

A total of 8 animals were used for quantitative real-time PCR and included 4 control animals and 4 animals exposed to blast using the 0.005 membrane. Total RNA was extracted from the brain tissue (cortex, striatum, hippocampus, and cerebellum) of saline perfused animals using Trizol reagent (Invitrogen) according to the manufacturer's instructions. Sample concentration was determined by spectral absorption and the purity confirmed using a 260/280 ratio of 1.8-2.0. Samples of cDNA were prepared via reverse transcription using commercially available kits (Applied Biosystems). Sample reactions included MultiScribe TM Reverse Transcriptase and 
random primers, with thermal cycle conditions set as follows: step 1 at $25^{\circ} \mathrm{C}$ for 10 minutes, step 2 at $37^{\circ} \mathrm{C}$ for 120 minutes, step 3 at $85^{\circ} \mathrm{C}$ for 5 seconds, and step 4 at $4^{\circ} \mathrm{C}$ for 10 minutes. For PCR amplification, TaqMan ${ }^{\circledR}$ Universal PCR Master Mix and the following probes were obtained from Applied Biosystems: 18s (Hs99999901_s1) for use as an endogenous control gene, GFAP (Rn00566603_m1), and Iba-1 (Rn00574125_g1). The reaction mixture was prepared based on manufacturer's instructions and the following thermal cycling conditions used: initial holding at $50^{\circ} \mathrm{C}$ for 2 minutes to allow for optimal AmpErase ${ }^{\circledR}$ UNG activity, followed by a first denaturing step at $95^{\circ} \mathrm{C}$ for 10 minutes, then 45 cycles at $95^{\circ} \mathrm{C}$ for 15 seconds, and at $60^{\circ} \mathrm{C}$ for 1 minute. Changes in gene expression were determined using the $\Delta \Delta \mathrm{C}_{\mathrm{T}}$ method and a threshold value of 0.2. Threshold cycle values of each gene were normalized to 18s rRNA.

Data Analysis

Data were analyzed using GraphPad Prism 5.0 (GraphPad Software, Inc.). A one-way ANOVA with posthoc Tukey's was used to compare across control and various blast-exposed groups. For gene expression work a student's t-test was utilized for comparing control and the blast-exposure group $(0.005$ membrane). A $\mathrm{P}<0.05$ was considered statistically significant for all data analyzed.

\section{RESULTS}

\section{Blast Model Design}

The model is capable of producing a characteristic Friedlander-type blast across a range of peak overpressures with short duration of exposure. The ideal blast wave in an open area, also known as the Friedlander curve, consists of a near-instantaneous pressure rise followed by an exponential decay of the overpressure (Bass et al., 2012). With all membranes tested utilizing the 
shock tube apparatus (Figure 2.1), ideal blast waves were obtained with a representative curve shown (Figure 2.2a). Peak overpressure increased in a linear fashion with membrane thickness with both reflected $\left(r^{2}=0.92\right)$ and incident $\left(r^{2}=0.93\right)$ sensor orientations allowing for the simulation of multiple peak overpressures in the experiment (Figure 2.2b,c).

\section{Injury Severity}

High peak overpressure blast, even of short duration, is associated with high mortality and extensive intracranial bleeds. While reflected pressures of $31.47 \pm 3.049$ and $50.72 \pm 3.368$ PSI failed to produce any mortality, exposure to $72.05 \pm 1.212$ PSI blast was associated with mortality in 3 of 9 experimental animals (33\%) while $90.10 \pm 1.030$ PSI blast exposures produced mortality in all 3 animals tested (100\%). Surviving animals failed to exhibit large hematomas at 72 hours post-blast (Figure 2.3a,b) but evidence of microvascular dysfunction and the potential for intraparenchymal bleeds was present (Figure 2.3c,d). Animals exposed to survivable moderate peak overpressures in this work did display small hematomas immediately following blast exposure (Figure 2.3c), consistent with the work of Reneer and colleagues (Reneer et al., 2011). Mortality was associated with the production of intracranial bleeds in the cortex, particularly on the contrecoup side as well as in the brainstem (Figure 2.3e,f). Similar findings have been observed utilizing explosives such as TNT in rat models in which animals placed in close range exhibit increased mortality rates associated with brain contusions, lacerations, and hematomas (Cheng et al., 2010).

\section{Vascular Injury}

Blast-induced brain injury results in the presence of intraparenchymal red blood cells, swollen neurons, and activated microglia. To further examine the effect of blast exposure on neural injury and microvascular dysfunction, particularly considering the presence of hematomas and 
parenchymal blood on gross examination, brain sections underwent hematoxylin and eosin (H\&E) staining. The intraparenchymal blood was confirmed with H\&E staining and red blood cells have clearly passed into the parenchyma and are no longer confined to the vasculature (Figure 2.4a). Evidence of neural injury and swollen, red axons was apparent in some animals (Figure 2.4b). The presence of red neurons indicates a potential anoxic/ischemic insult (de Lanerolle et al., 2011), consistent with the presence of microvascular dysfunction observed following blast exposure (Svetlov et al., 2012). The presence of activated microglia, capable of releasing an array of inflammatory mediators, was also seen with blast exposure and prompted further investigation of astro- and microgliosis.

\section{Neurodegeneration}

Neural degeneration occurs in a dose-response manner consistent with blast severity. Blastinduced brain injury resulted in increased neural degeneration measured using Fluoro-Jade B staining in the S1BF region of the cortex on both the coup $(F(3,23)=47.03, P<0.0001)$ and contrecoup $(\mathrm{F}(3,23)=110.7, \mathrm{P}<0.0001)$ sides when analyzed by one-way ANOVA (Figure 2.5ad). Post hoc tests revealed that blast-induced brain injury produced increases in neural degeneration compared to control at $31.47 \pm 3.049$ PSI on the contrecoup side $(q=4.51, P<0.05)$, $50.72 \pm 3.368$ PSI on both $\operatorname{coup}(\mathrm{q}=10.29, \mathrm{P}<0.0001)$ and contrecoup $(\mathrm{q}=13.69, \mathrm{P}<0.0001)$ sides, and 72.05 \pm 1.212 PSI on both coup $(q=15.13, \mathrm{P}<0.001)$ and contrecoup $(\mathrm{q}=23.76, \mathrm{P}<$ 0.0001) sides (Figure 2.5e-f). Neural degeneration following blast exposure was also observed within other brain regions including the striatum, hippocampus, cerebellum and regions surrounding the corpus callosum (Supplemental Information).

\section{Glial Reactivity}


Blast injured brains demonstrate increased glial activation with increasing peak overpressure. Reactive astrocytes, identified by GFAP staining, were elevated significantly within the corpus callosum on the coup $(\mathrm{F}(3,23)=26.08, \mathrm{P}<0.0001)$ and contrecoup $(\mathrm{F}(3,23)=8.03, \mathrm{P}<$ 0.001) sides of the brain following blast-induced neurotrauma, consistent with findings from other blast injury models (Svetlov et al., 2012), when analyzed using one-way ANOVA (Figure 2.6a-d). Post hoc tests showed that blast injury resulted in elevated astrocyte reactivity at $50.72 \pm 3.368$ PSI on the contrecoup side $(\mathrm{q}=5.13, \mathrm{P}<0.01)$ and at $72.05 \pm 1.212$ PSI on both the coup $(\mathrm{q}=$ 11.28, $\mathrm{P}<0.0001)$ and contrecoup $(\mathrm{q}=6.03, \mathrm{P}<0.01)$ sides (Figure 2.6e-f).

Similar findings were observed concerning microglial activation, identified with Iba-1 staining, with increased levels of microglial activation relative to control on both coup $(\mathrm{F}(3,23)=$ 83.12, $\mathrm{P}<0.0001)$ and contrecoup $(\mathrm{F}(3,23)=25.37, \mathrm{P}<0.0001)$ sides (Figure 2.7a-d). Again, although microglial presence was detected throughout the brain and appeared increased throughout, microglial activation was most prominent throughout the corpus callosum and internal capsule, consistent with other studies (de Lanerolle et al., 2011). Notably, microglia appeared more rounded or amoeboid, typical of phagocytic microglia, within these white matter tracts consistent with white matter damage observed in human subjects following trauma (Johnson et al., 2013c). Previous reports in swine exposed to blast and assessed primarily at 2 weeks failed to identify extensive microglial activation, a potential reason for the discrepancy between studies (de Lanerolle et al., 2011). Post hoc tests revealed increased microglial activation following $31.47 \pm$ 3.049 PSI blast on the contrecoup side $(\mathrm{q}=4.71, \mathrm{P}<0.05), 50.72 \pm 3.368$ PSI blast on both coup $(\mathrm{q}=8.37, \mathrm{P}<0.0001)$ and contrecoup $(\mathrm{q}=7.85, \mathrm{P}<0.0001)$ sides, and $72.05 \pm 1.212$ PSI blast on both coup $(\mathrm{q}=20.49, \mathrm{P}<0.0001)$ and contrecoup $(\mathrm{q}=11.92, \mathrm{P}<0.0001)$ sides as well (Figure 2.7e-f). The clear neuroinflammatory response, characterized by a graded glial response based on 
peak overpressure exposure, observed in this work is consistent with findings by Cernak and colleagues in which mild and moderate blast exposure produced increases in GFAP, as well as ED1, a marker of microglia, at numerous time points (Cernak et al., 2011). Importantly, activated microglia in other blast exposure studies have been associated with changes in neuron appearance such as darkened dendrites (Kaur et al., 1995) and in vitro work has demonstrated a mild activation of microglia following overpressure exposure with pulse duration altering microglial gene expression (Kane et al., 2012).

Gene expression of GFAP (Figure 2.8a) was increased at 24 hours following blast exposure in the cortex $(\mathrm{t}=2.412, \mathrm{P}<0.05)$ but not in the striatum $(\mathrm{t}=0.022, \mathrm{P}>0.05)$, hippocampus $(\mathrm{t}=$ 1.084, $\mathrm{P}>0.05)$, or cerebellum $(\mathrm{t}=0.634, \mathrm{P}>0.05)$. The lack of difference in GFAP gene expression in regions outside of the cortex was in contrast to the visible differences seen in these regions at 72 hours based on immunohistochemistry (Supplemental Information). The reason for this discrepancy is not entirely clear but may be due to consideration of the entirety of brain sections for gene expression studies when immunohistochemistry reveals findings largely located at the interface of regions and the gray-white matter junction. Another possibility explaining the discrepancy between immunohistochemical and gene expression findings is the time of analysis. Only one time point for each assay was included and therefore, changes in gene expression could have occurred rapidly following blast and already declined to baseline by 24 hours. No difference in Iba-1 gene expression (Figure 2.8b) was detected in the cortex $(\mathrm{t}=0427, \mathrm{P}>0.05)$, striatum $(\mathrm{t}$ $=0.900, \mathrm{P}>0.05)$, hippocampus $(\mathrm{t}=0.380, \mathrm{P}>0.05)$, or cerebellum $(\mathrm{t}=0.448, \mathrm{P}>0.05)$, consistent with immunohistochemical findings (Supplemental Information).

\section{Locomotor Activity}


Blast-induced traumatic brain injury produces deficits in total activity regardless of severity. Total activity of the experimental subjects was decreased at 24 hours post-blast in all injured groups. The total activity level post-injury did not vary based on injury severity with all injury groups demonstrating similar impairments (Figure 2.9a). The deficits in total activity were due to a decrease in ambulatory activity associated with blast exposure (Figure 2.9b). Blast exposure did not alter measures of fine activity (Figure 2.9c).

\section{DISCUSSION}

Efforts to model blast injury have been plagued by the lack of standardization across laboratories and the appropriate scaling of blast parameters to the animal species used based on data obtained from human studies. Preclinical studies are varied with regards to animal placement within or outside of the shock tube, orientation to the blast front, protection of the thorax, and blast parameters, namely peak overpressure and duration of exposure. Studies in soldiers revealed common IED exposures producing blast injury result from detonation of 105-mm and 155-mm artillery rounds at a distance of 5-10 meters. Use of the Conventional Weapons Effects Program (CONWEP) (Hyde, 2004) allows for interpretation of these exposures in relation to blast wave parameters with a peak overpressure of 50-1000 kPA (7.25-145.03 PSI) and an exposure duration of 2-10 ms (Panzer et al., 2012). As such, utilizing these parameters and scaling appropriately to preclinical animal species used may represent a more clinically relevant blast exposure and allow for improved elucidation of blast-induced injury pathophysiology and evaluation of proposed therapeutics.

The concept of scaling blast injury to animal models is based largely upon previous works assessing pulmonary injury and primary blast survival across animal species. Richmond and 
colleagues identified, in various species, the effect of blast duration on lethality with longer duration blasts requiring a lower peak overpressure to produce mortality when compared to short duration blasts that required a significantly higher peak overpressure (Richmond et al., 1968). A clear relationship between mass and blast parameters associated with lethality exists, particularly with regards to the more clinically relevant short duration blast (Richmond et al., 1968). In fact, classical work by Bowen and colleagues investigated pulmonary injury in relation to blast exposure and created a model system in which scaling is based upon the height of burst divided by the cubed root of the mass (Bowen et al., 1968). This has been simplified to an equation utilizing the cubed root of the reference mass (human) divided by mass of the experimental subject (Bass et al., 2012; Panzer et al., 2012; Shridharani et al., 2012). The scaling equations developed have displayed significant utility in development of injury risk functions based upon the high level of agreement with pre-existing experimental data concerning blast survival and injury risk. As such, while scaling of blast injury and associated blast parameters based on subject weight has not been related to neural injury (Park et al., 2011), scaling represents a logical step forward in more closely replicating clinically relevant exposures in laboratory models (Panzer et al., 2012; Rafaels et al., 2012; Risling and Davidsson, 2012).

To address the question of how scaling affects blast-induced brain injury a shock tube capable of producing a range of peak overpressures with short durations was designed and utilized within this work. Notably, the model produced clear neuropathological changes indicative of neural degeneration and glial reactivity. These findings were consistent with work by Goldstein and colleagues in which single-blast exposure produced neurodegenerative changes consistent with chronic traumatic encephalopathy (CTE) (Goldstein et al., 2012) as well as other work utilizing scaling principles (Shridharani et al., 2012). Similarly, neuropathological changes were 
observed throughout the cortex as previously described (Goldstein et al., 2012) but were most prominent with the corpus callosum and internal capsule, consistent with previous findings in other blast injury models (Park et al., 2011). It remains unclear why specific brain regions may be more susceptible to blast-induced brain injury but one logical explanation is that of spallation. Spallation refers to the interface-based disruption that occurs between tissues of different densities upon a compression wave in the denser medium reflecting at the interface resulting in displacement and fragmentation of the denser medium into the less dense medium (Cernak and Noble-Haeusslein, 2010; Covey and Born, 2010; Yeh and Schecter, 2012). Spalling has been identified as a leading cause of endothelial injury and activation of microglia (Duckworth et al., 2012), consistent with our findings. Similarly, endothelial damage results in a loss of tight junction integrity and consequently the potential for increased blood-brain barrier permeability and microvascular injury (Duckworth et al., 2012). Notably, increased BBB permeability has been found as late as one month following blast-induced brain injury in a preclinical rodent model, indicating the presence of lasting microvascular dysfunction (Rubovitch et al., 2011b). Furthermore, blast-induced BBB disruption has been observed to occur more extensively on the contralateral side, as well as at fluid-tissue interfaces such as that surrounding the lateral ventricles (Readnower et al., 2010), consistent with the findings reported within this study in which the contralateral side was more severely injured in comparison to the ipsilateral side based on standard markers of neural degeneration, astrocyte reactivity, and microglial activation (Garman et al., 2011).

In addition to the neuropathological findings, the model utilized in this work produced deficits in total activity at 24 hours post-blast, regardless of experimental group. How blast-injury models affect behavioral functions in rodents is poorly understood but of clear interest based on clinical findings associated with blast that include retrograde amnesia, confusion, loss of 
conscioussness, depression, post-traumatic stress disorder (PTSD), vertigo, balance disorders, anxiety, apathy, and difficulty concentrating and performing executive functions (Taber et al., 2006; Rubovitch et al., 2011b; Magnuson et al., 2012; Risling and Davidsson, 2012; Scheibel et al., 2012). Previous reports have demonstrated impairment on the accelerating rotarod following direct cranial blast injury (Kuehn et al., 2011) and on the balance beam following whole body blast exposure (Ahlers et al., 2012) while others have shown that shielding of the torso prevents neural injury and associated behavioral deficits following blast (Koliatsos et al., 2011). Cognitive abilities, particularly reference memory, may also be impaired following blast injury. The latency to find the platform using the Morris Water Maze may be related to and correlate with maximum peak overpressure exposure (Saljo et al., 2011). Other hippocampal-based learning and memory tasks such as the Barnes maze and Y-maze have also been utilized successfully in the study of functional deficits induced post-injury with blast subjects performing significantly worse in both acquisition and memory tasks (Koliatsos et al., 2011; Goldstein et al., 2012). The same can be said of the passive avoidance task with animals showing anterograde memory deficits but not retrograde amnesia following blast-induced brain injury (Ahlers et al., 2012). Previous studies have also revealed deficits following whole-body blast injury by assessing memory and social interaction through the social recognition memory test (Koliatsos et al., 2011). Evaluation of cognitive abilities at 30 days post-blast in a mouse model has revealed persistent deficits in the novel object recognition test as well (Rubovitch et al., 2011b). This serves as one functional test capable of detecting sustained deficits following blast injury. Other assessments capable of detecting neural injury in the chronic period following blast exposure include the accelerating rotarod, active avoidance, and open field activity (Cernak et al., 2011). Furthermore, the novel object recognition test appears exceptionally sensitive to neural dysfunction and memory 
impairments, even in the absence of histological changes following blast exposure as described by Baalman, et al (Baalman et al., 2012). While the significance of these findings is unclear and how these findings translate from one model to another, let alone to the clinical population, there is a clear need for further studies in standardized, clinically-relevant models.

It is clear that future studies, in addition to understanding the effect of scaled blast exposure on functional ability and behavior-related changes, need to better characterize the inflammatory processes associated with blast exposure and how these relate to neural injury and neurodegeneration. While it is unlikely blast exposure can be completely prevented, particularly in civilians exposed to terrorist-type attacks, it may be possible to improve therapeutic options. The need for improved therapeutics is particularly clear in the military in which evacuation of blast-exposed troops is often delayed by hours to several days due to the nature of conflict. As such, secondary responses within the brain often occur and may represent a potential target as described previously (Dalle Lucca et al., 2012). 


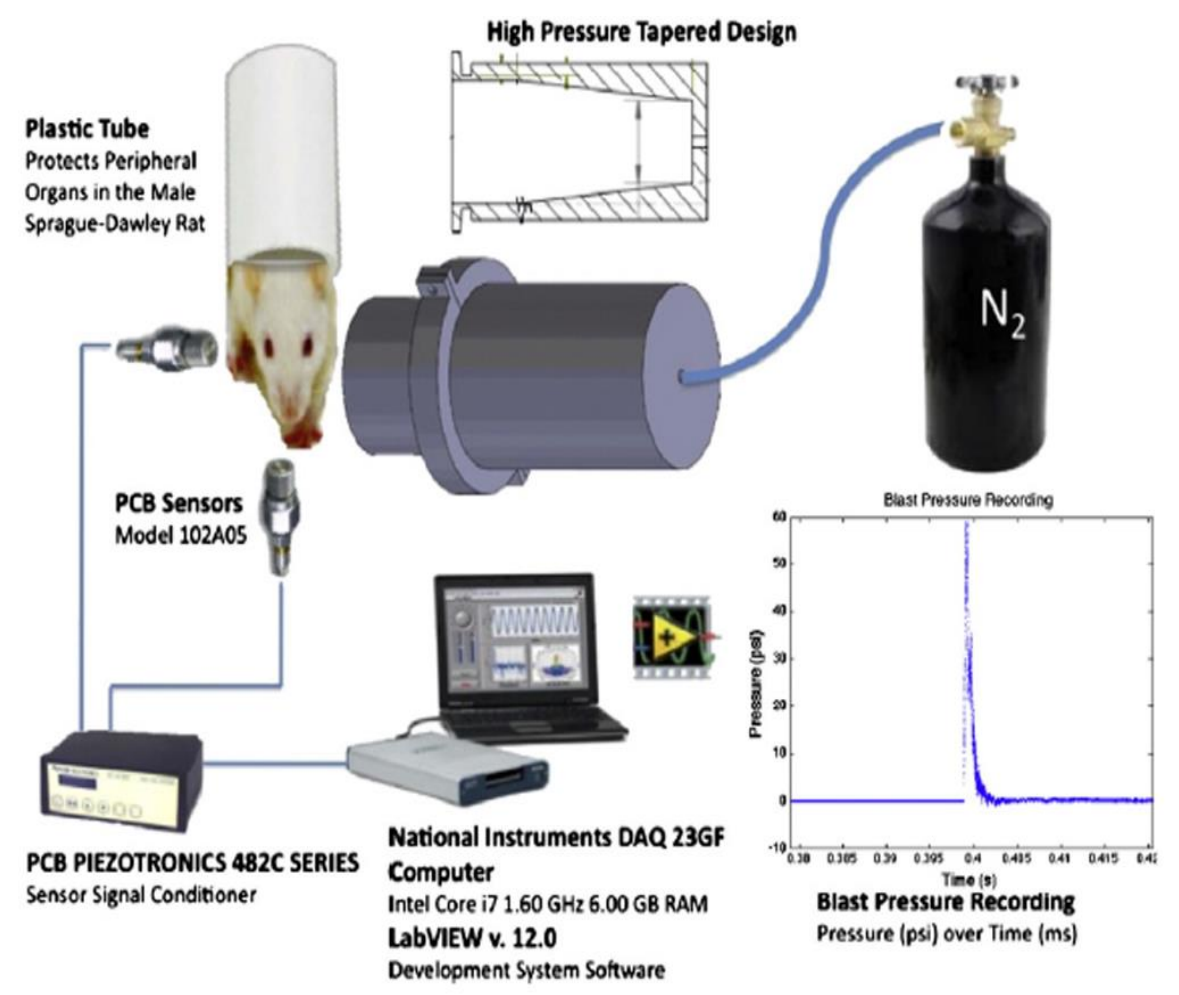

Figure 2.1. Experimental setup utilized in this work demonstrating the novel shock tube apparatus for simulating blast-induced brain injury and data acquisition procedures. The shock tube utilizes a tapered driver section to reduce wave reflection and alterations of the shock wave by the expansion wave. The driver section was kept short to reduce the volume of gas required to produce membrane rupture and therefore, reduced overpressure duration and impulse. The abdomen and thorax of animals was protected using a rigid apparatus. 

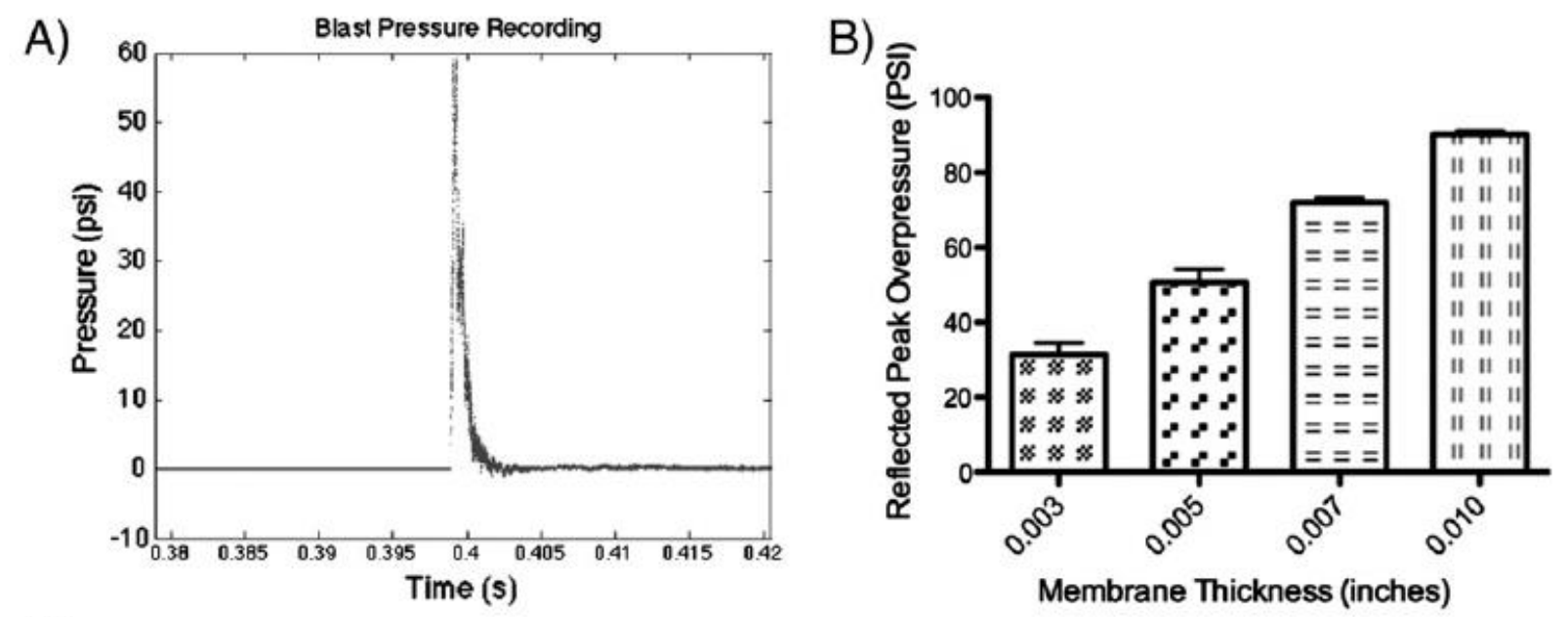

C)

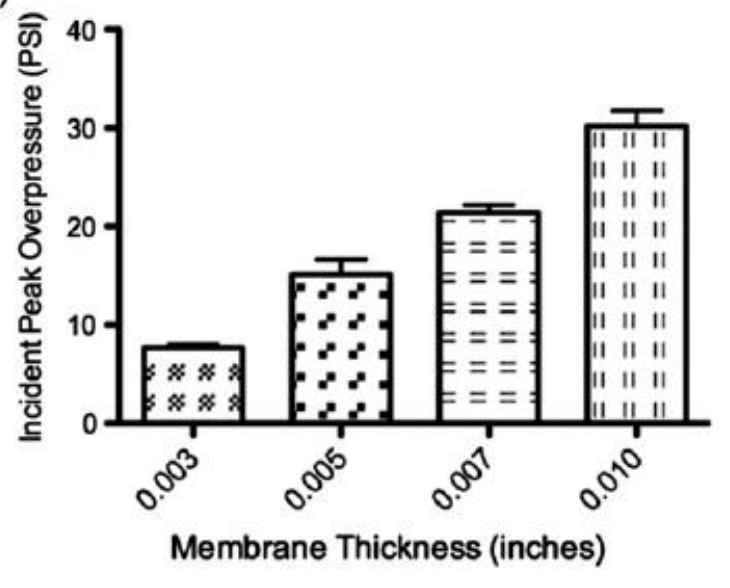

Figure 2.2. A.) Sample shock wave recording (reflected sensor) representing a classical Friedlander wave of short duration. B.) Peak reflected overpressure recorded as a function of membrane thickness. C.) Peak incident overpressure recorded as a function of membrane thickness. 


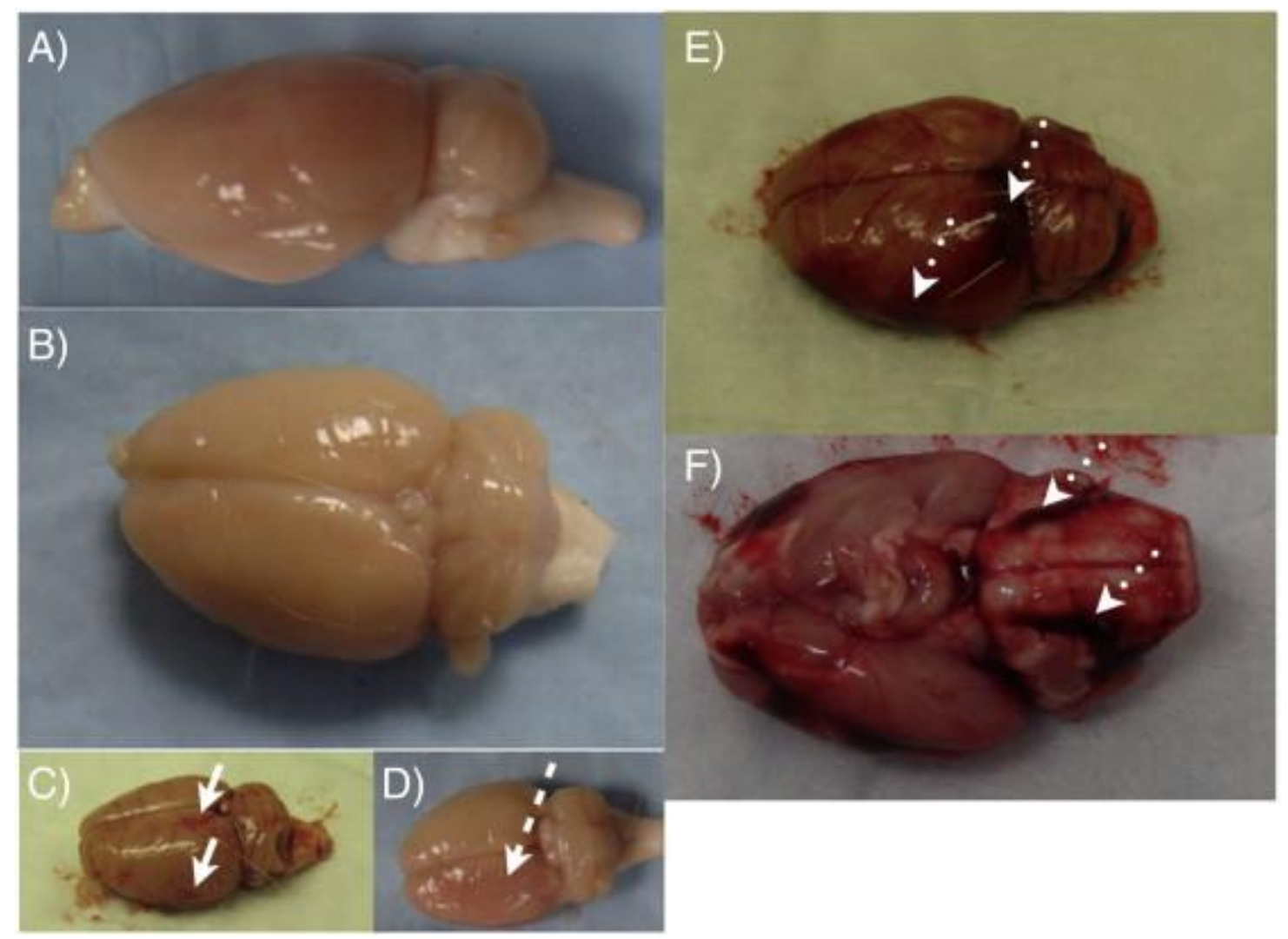

Figure 2.3. Gross examination of single blast-exposed brains. A.) Brain of a perfused control animal, B.) $31.47 \pm 3.049$ PSI (reflected) blast-exposed brain at 72 hours post-blast following perfusion demonstrating no apparent hematomas or gross changes, C.) Presence of hematomas and contusion immediately following exposure to $50.72 \pm 3.368$ PSI (reflected) blast on the contrecoup side, D.) At 72 hours post-blast, 72.05 \pm 1.212 PSI (reflected) blast exposure results in the appearance of parenchymal blood and an overall husky appearance on the contrecoup side, E.) Immediately following 90.10 \pm 1.030 PSI (reflected) blast, clear evidence of bleeds on the cortex, and F.) Immediately following $90.10 \pm 1.030$ (reflected) PSI blast, brainstem bleeds were apparent and associated with mortality. 

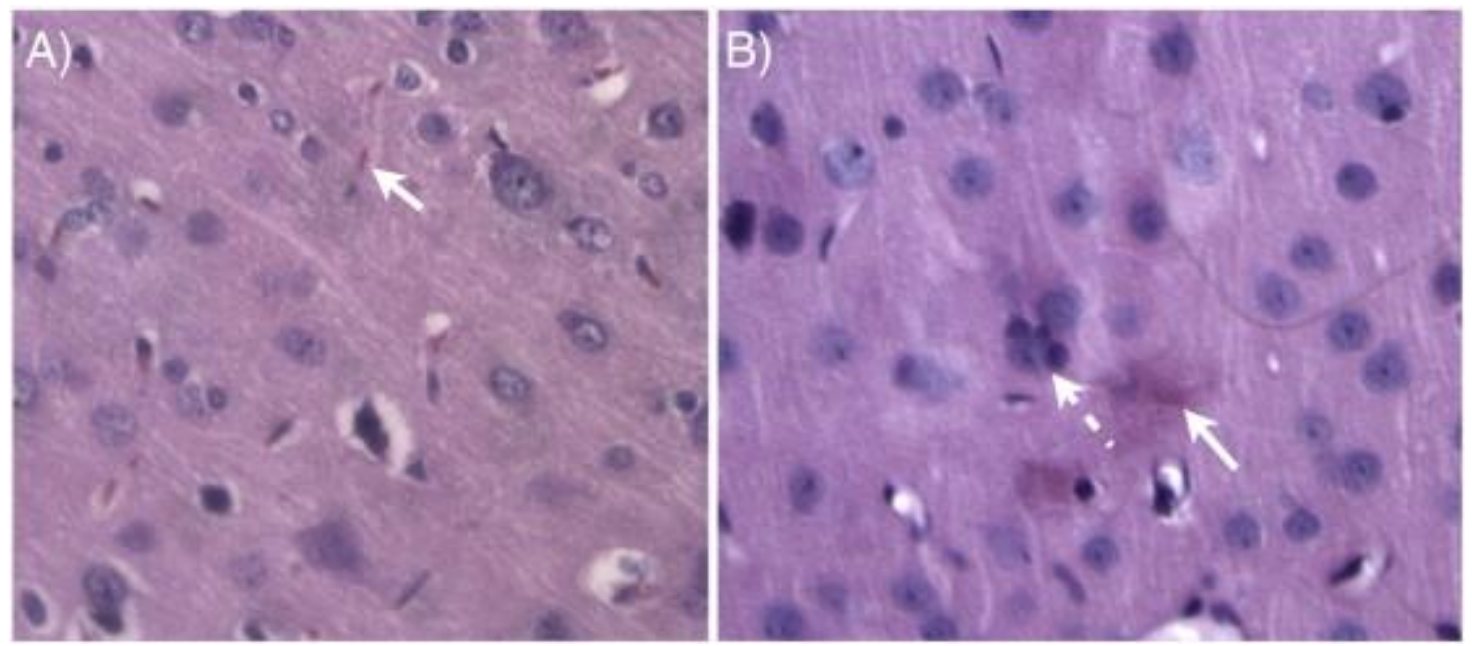

Figure 2.4. Blast exposure, particularly at high peak overpressures, was associated with microvascular damage and neural injury on H\&E staining. A.) Presence of red blood cells within the parenchyma at 72 hours following $72.05 \pm 1.212$ PSI (reflected) blast. B.) Swollen, red neuron at 72 hours following $72.05 \pm 1.212$ (reflected) PSI blast. Red neurons typically are associated with anoxic/ischemic injury indicating the presence of secondary damage, likely related to microvascular dysfunction, following blast injury. 

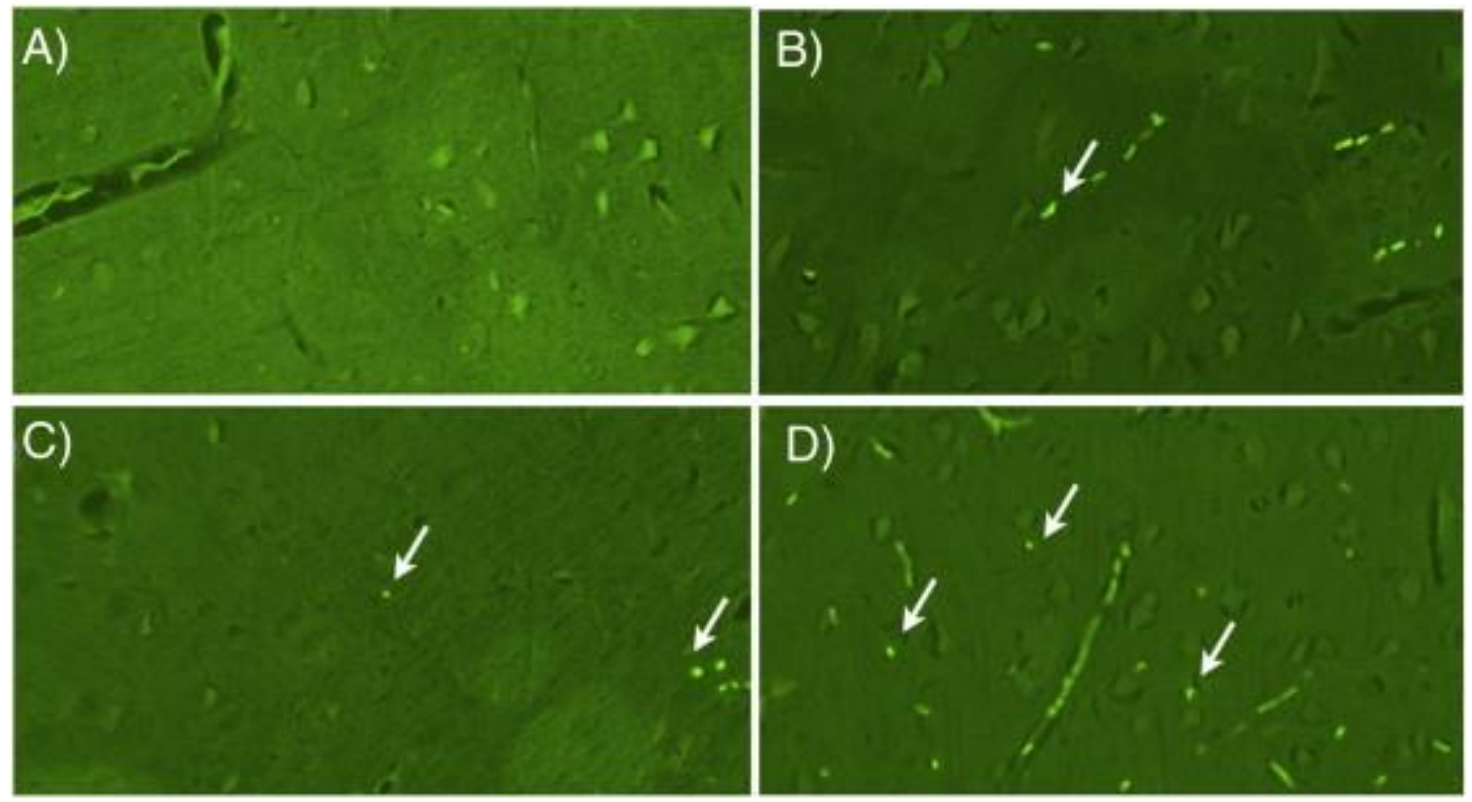

E)

F)
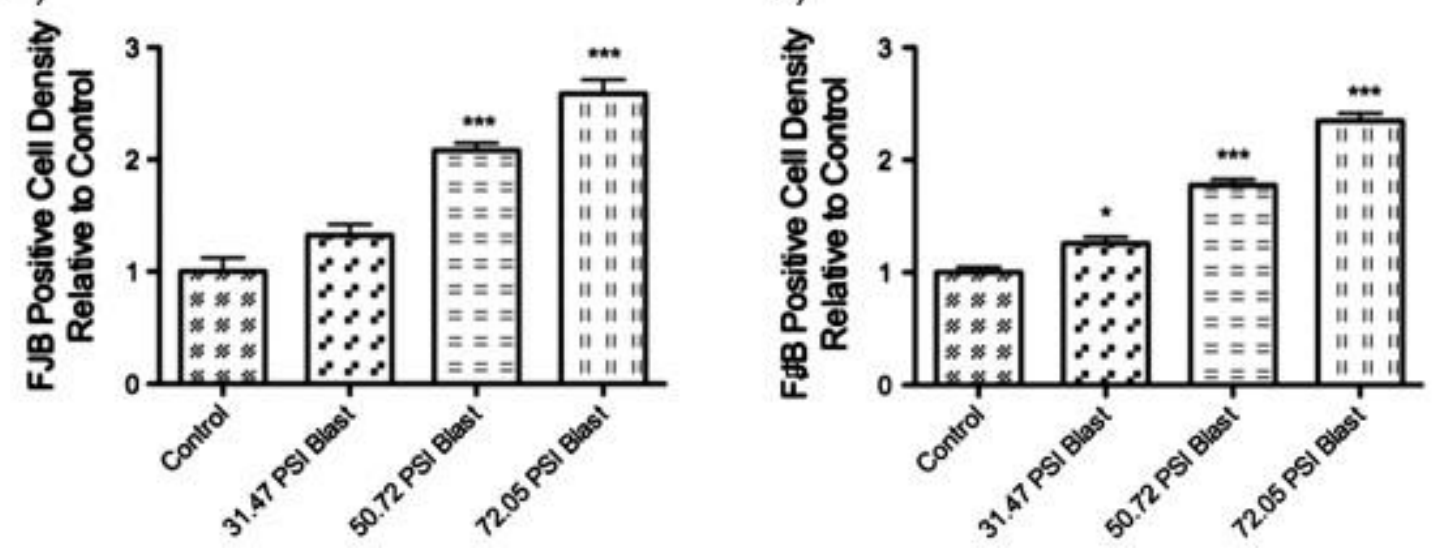

Figure 2.5. Blast exposure produced neural degeneration within the S1BF region of the cortex at 72 hours post-exposure as indicated with fluoro-jade B staining. A.) $31.47 \pm 3.049$ PSI (reflected) blast on the coup side, B.) $50.72 \pm 3.368$ PSI blast on the coup side, C.) $31.47 \pm 3.049$ PSI blast on the contrecoup side, and D.) $72.05 \pm 1.212$ PSI blast on the contrecoup side. Quantification of Fluoro-Jade B (FJB) positive neurons using stereology demonstrated a graded response based on peak overpressure exposure in the $\mathrm{S} 1 \mathrm{BF}$ region of the cortex on $\operatorname{coup}(\mathrm{F}(3,23)=47.03, \mathrm{P}<0.0001)$ and contrecoup $(\mathrm{F}(3,23)=110.70, \mathrm{P}<0.0001)$ sides $(\mathbf{E}$ and $\mathbf{F}$, respectively). Post hoc analysis showed that blast exposure resulted in increased neural degeneration relative to control with 31.47 \pm 3.049 PSI on the contrecoup side $(\mathrm{q}=4.51, \mathrm{P}<0.05), 50.72 \pm 3.368$ PSI on both coup $(\mathrm{q}=$ $10.29, \mathrm{P}<0.0001)$ and contrecoup $(\mathrm{q}=13.69, \mathrm{P}<0.0001)$ sides, and $72.05 \pm 1.212$ PSI on both coup $(\mathrm{q}=15.13, \mathrm{P}<0.001)$ and contrecoup $(\mathrm{q}=23.76, \mathrm{P}<0.0001)$ sides. 

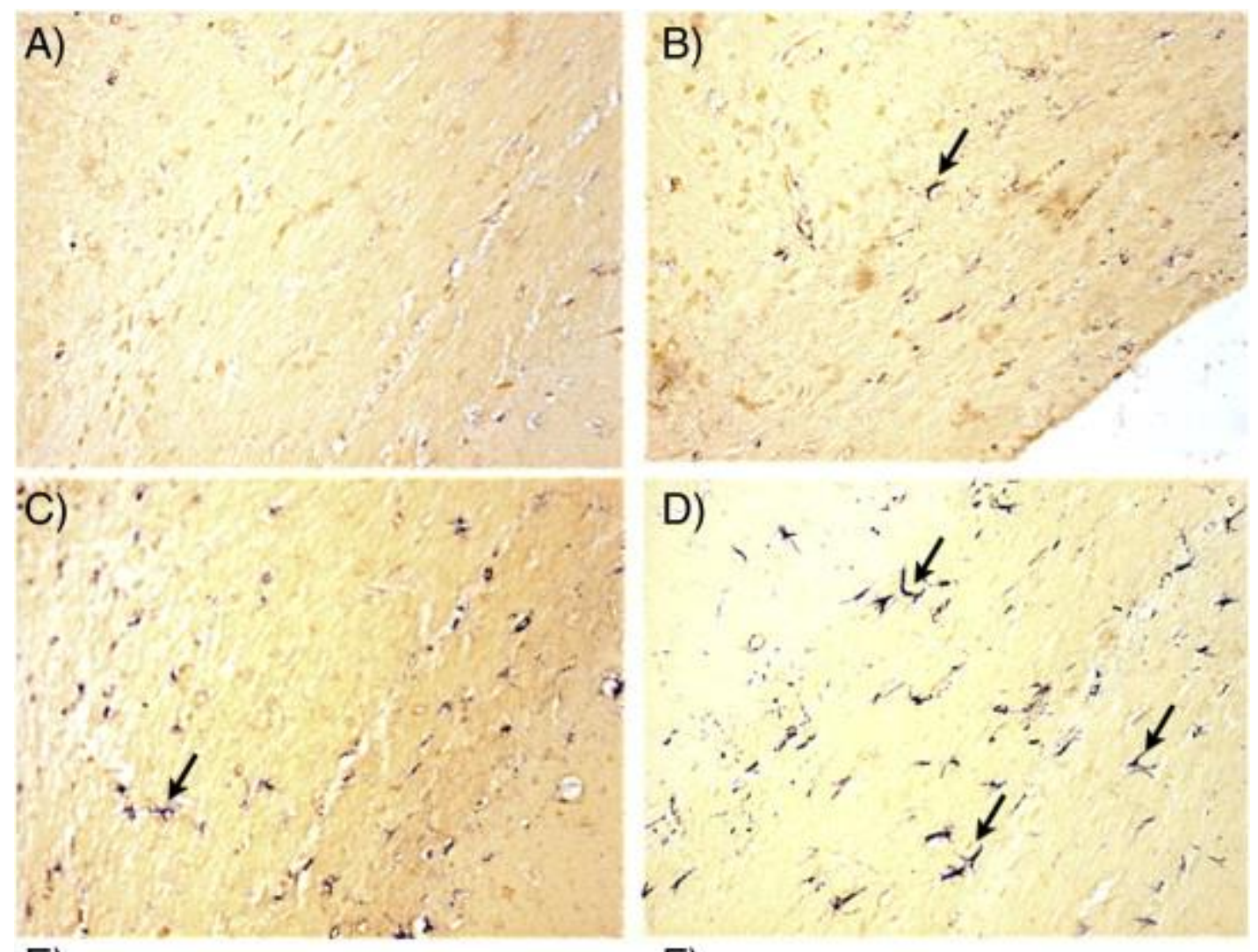

E)

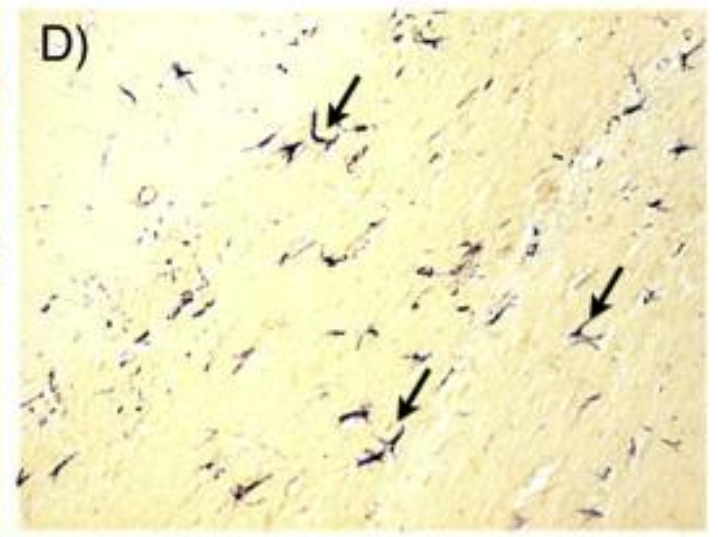

F)
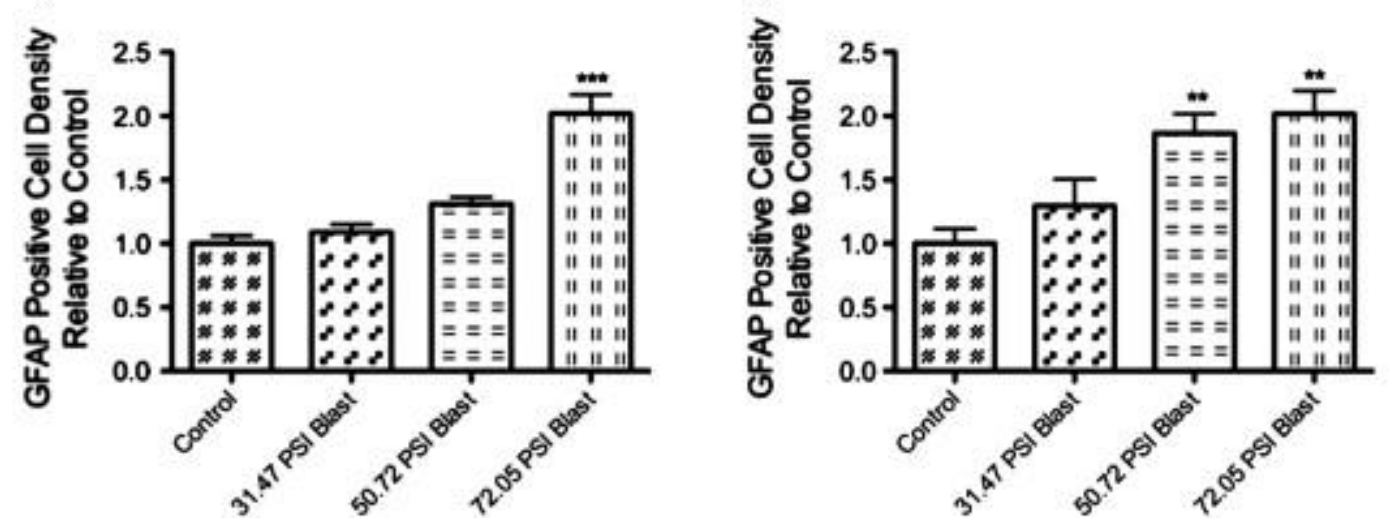

Figure 2.6. Blast exposure produced extensive astrocyte reactivity within the corpus callosum at 72 hours post-exposure as indicated with glial fibrillary acidic protein (GFAP) staining. A.) 31.47 \pm 3.049 PSI blast on the coup side, B.) $72.05 \pm 1.212$ PSI blast on the coup side, C.) control (no blast exposure) on the contrecoup side, and D.) $72.05 \pm 1.212$ PSI blast on the contrecoup side. Quantification of Glial Fibrillary Acidic Protein (GFAP) positive astrocytes using stereology demonstrated a graded response based on peak overpressure exposure in the corpus callosum on the coup $(F(3,23)=26.08, P<0.0001)$ and contrecoup $(F(3,23)=8.03, P<0.001)$ sides $(E$ and $F$, respectively). Post hoc analysis showed that blast exposure resulted in increased astrocyte reactivity relative to control with $50.72 \pm 3.368 \mathrm{PSI}$ on the contrecoup side $(q=5.13, P<0.01$ ) and at $72.05 \pm 1.212 \mathrm{PSI}$ on both the coup $(\mathrm{q}=11.28, \mathrm{P}<0.0001)$ and contrecoup $(\mathrm{q}=6.03, \mathrm{P}<$ 0.01 ) sides. 

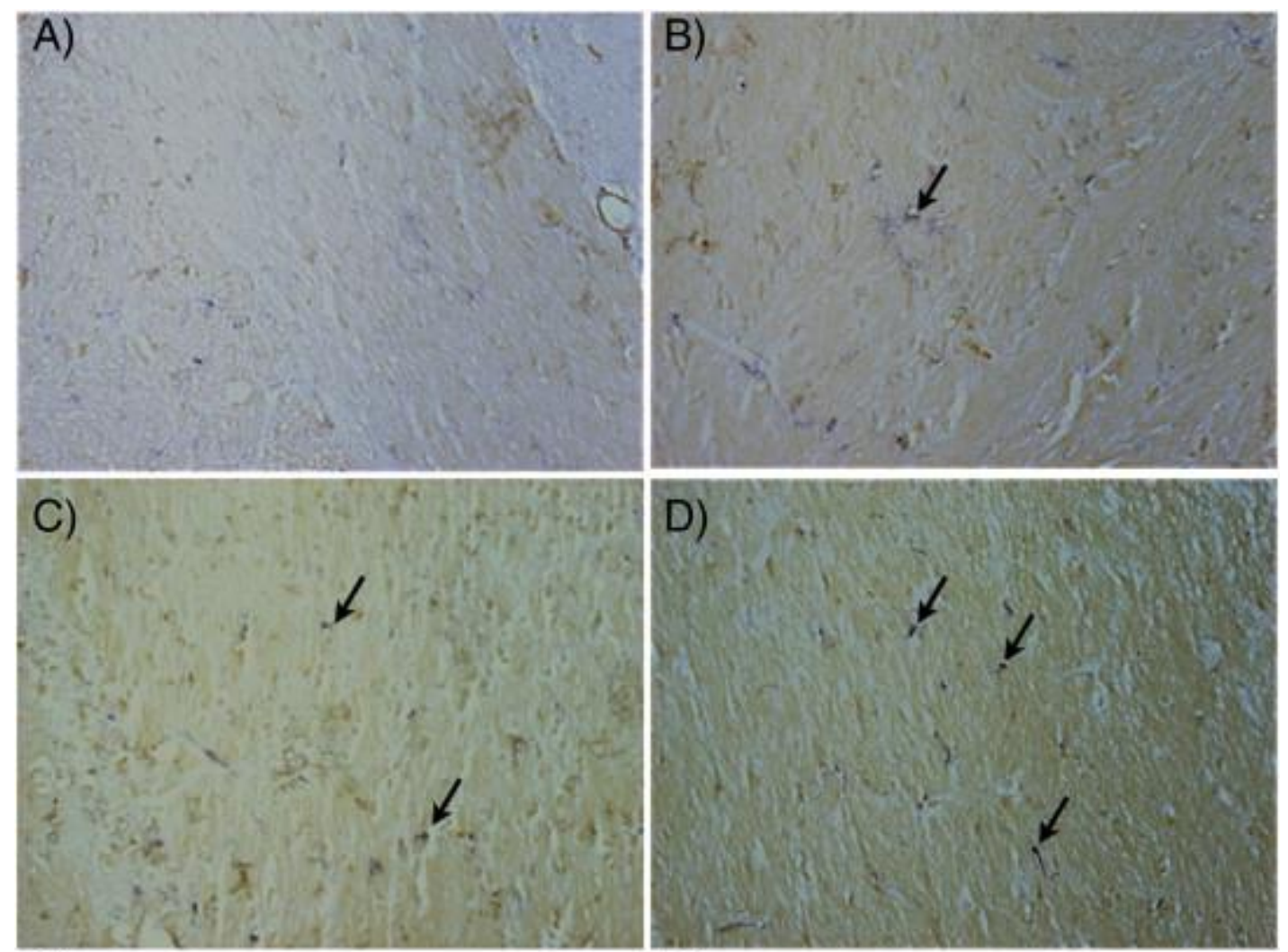

E)

F)
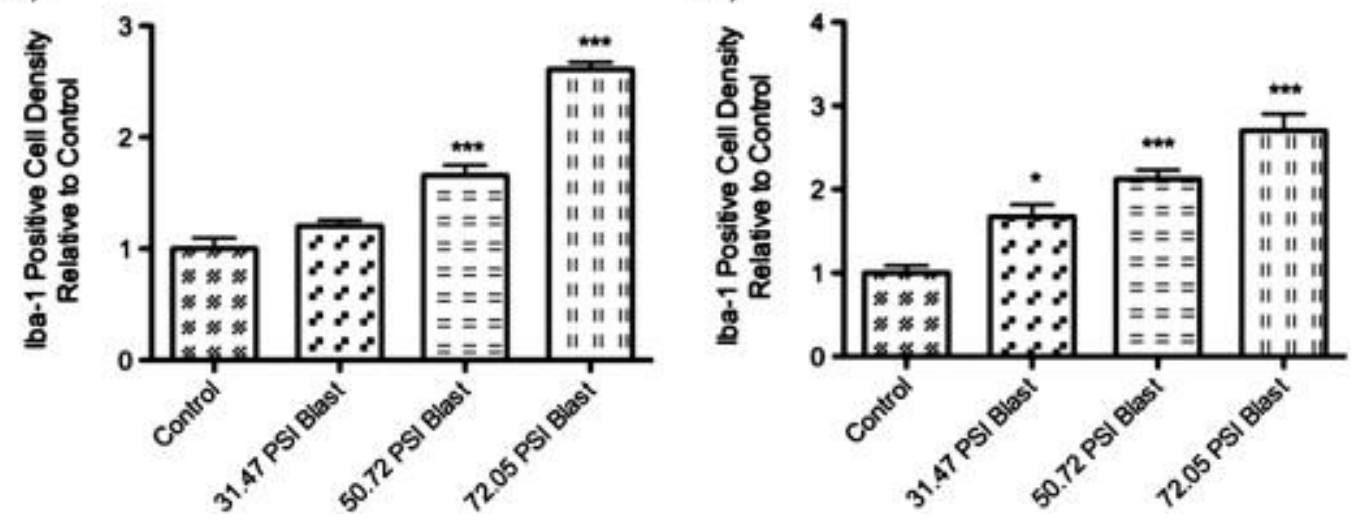

Figure 2.7. Blast exposure produced microglial changes within the corpus callosum at 72 hours post-exposure as indicated with ionized calcium-binding adapter molecule 1 (Iba-1) staining. A.) Control (no blast exposure) on the coup side, B.) $50.72 \pm 3.368$ PSI blast on the coup side, C.) $31.47 \pm 3.049$ PSI blast on the contrecoup side, and D.) $72.05 \pm 1.212$ PSI blast on the contrecoup side. Quantification of Ionized Calcium-binding Adapter Molecule 1 (Iba-1) positive microglia using stereology demonstrated a graded response based on peak overpressure exposure in the corpus callosum on the coup $(\mathrm{F}(3,23)=83.12, \mathrm{P}<0.0001)$ and contrecoup $(\mathrm{F}(3,23)=25.37$, $\mathrm{P}<0.0001$ ) sides ( $\mathbf{E}$ and $\mathbf{F}$, respectively). Post hoc analysis showed that blast exposure resulted in increased microglial activation relative to control with $31.47 \pm 3.049$ PSI blast on the contrecoup side $(\mathrm{q}=4.71, \mathrm{P}<0.05), 49$ PSI blast on both coup $(\mathrm{q}=8.37, \mathrm{P}<0.0001)$ and contrecoup $(\mathrm{q}=$ $7.85, \mathrm{P}<0.0001)$ sides, and $72.05 \pm 1.212$ PSI blast on both coup $(\mathrm{q}=20.49, \mathrm{P}<0.0001)$ and contrecoup $(\mathrm{q}=11.92, \mathrm{P}<0.0001)$ sides as well. 

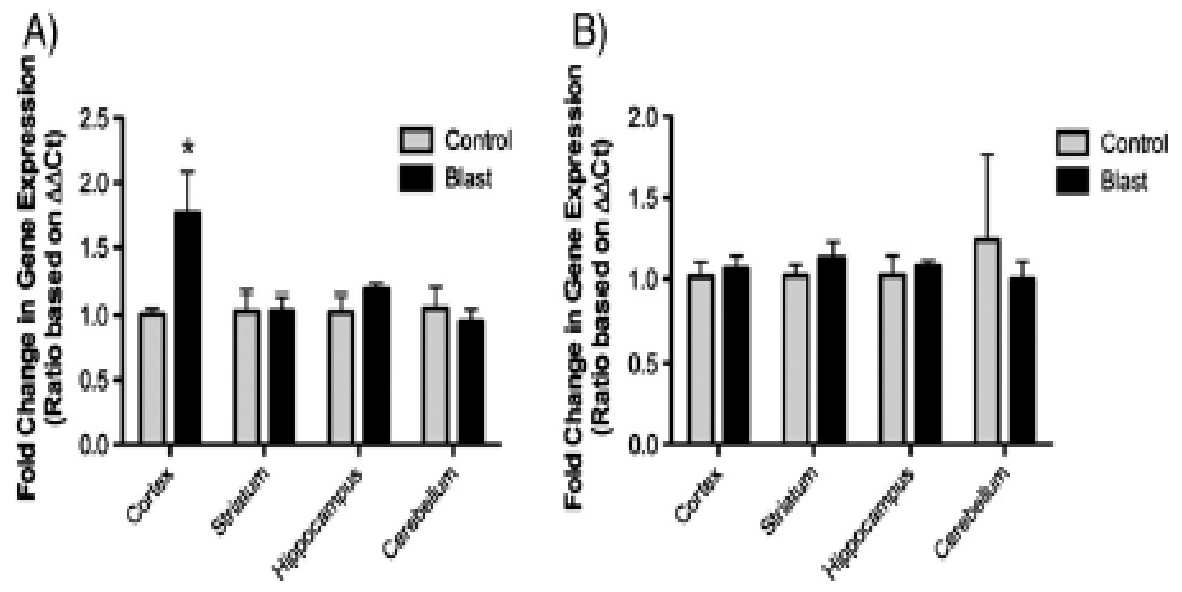

Figure 2.8. Effect of 50.72 PSI blast exposure on gene expression at 24 hours post-injury. A.) Gene expression of glial fibrillary acidic protein (GFAP) was increased within the cortex $(\mathrm{t}=$ 2.412, $\mathrm{P}<0.05$ ) but not in the striatum, hippocampus, or cerebellum. B.) Gene expression of ionized calcium binding adapter molecule (Iba-1) was not changed within any of the brain regions studied at 24 hours post-blast. 
A)

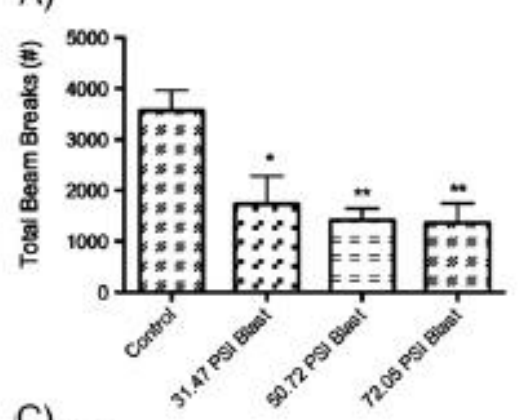

C)

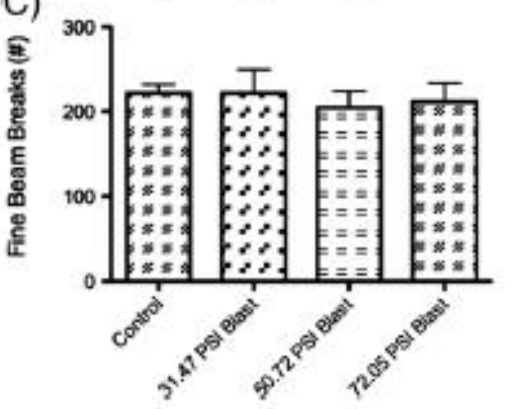

B)

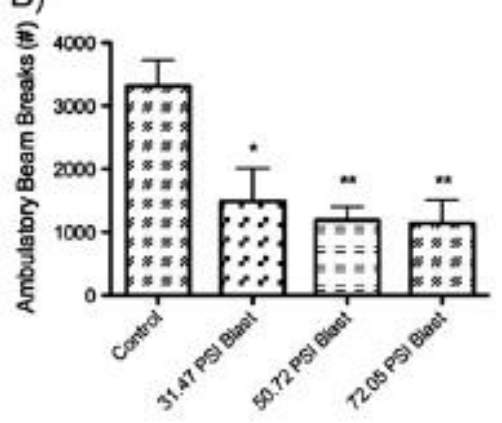

Figure 2.9. Effect of blast exposure on total, ambulatory, and fine activity levels at 24 hours postinjury. A.) Total activity was significantly altered with blast exposure $(\mathrm{F}(3,23)=6.55, \mathrm{P}<0.05)$ with post hoc analysis identifying a reduction relative to control with $31.47 \pm 3.049$ PSI blast $(\mathrm{q}=$ 4.50, $\mathrm{P}<0.05), 50.72 \pm 3.368$ PSI blast $(\mathrm{q}=5.28, \mathrm{P}<0.05)$, and $72.05 \pm 1.212$ PSI blast $(\mathrm{q}=5.40$, $\mathrm{P}<0.05)$. B.) Ambulatory activity was reduced with blast exposure $(\mathrm{F}(3,23)=6.74, \mathrm{P}<0.05)$ with post hoc analysis identifying a reduction relative to control with $31.47 \pm 3.049$ PSI blast $(\mathrm{q}=4.59$, $\mathrm{P}<0.05), 50.72 \pm 3.368$ PSI blast $(\mathrm{q}=5.34, \mathrm{P}<0.05)$, and $72.05 \pm 1.212$ PSI blast $(\mathrm{q}=5.47, \mathrm{P}<0.05)$ exposures. C.) Fine activity failed to demonstrate differences across any group comparisons $(\mathrm{F}(3,23)=0.17, \mathrm{P}>0.05)$. 


\section{Chapter Three}

Characterization of neural injury and neurobehavioral dysfunction in the rat after traumatic brain injury

This work is in preparation. 


\begin{abstract}
Traumatic brain injury resulting from blast exposure is the most common injury of military combatants. Animal modeling of blast-induced traumatic brain injury has demonstrated severe histopathological effects accompanied by loss of long-term synaptic plasticity and cognitive performance due to tissue damage. Here we use a scaled blast model that was specifically developed to assess the neuropathological, neurophysiological, and biochemical effects of exposure to blast forces not severe enough to cause overt physiological deficits or behavioral changes. We find that mild blast exposure induces no observable macroscopic histological effects, but instead causes microvascular injury, axonal damage, and neuroinflammation in various brain regions including hippocampus. Hippocampal deficits in short term plasticity and synaptic excitability were observed 7-days after mild blast exposure. Biochemical analysis revealed that mild blast exposure invokes markers of excitotoxicity and neuronal injury in hippocampus. Thus mild blast exposure causes subtle but deleterious neurological effects in the absence of major behavioral deficits, suggesting that subclinical exposures to blast forces may initiate neuropathology.
\end{abstract}




\section{INTRODUCTION}

Blast-induced traumatic brain injury (bTBI) occurs when a shock wave penetrates the skull and causes physical damage to neurons, glia and the vasculature. Explosion-derived shock waves have made bTBI the hallmark injury of American combatants (Huber et al., 2013). Diagnosis of bTBI is comorbid with increased incidence of neuropsychiatric disorders and long-term physical, cognitive, behavioral, and emotional changes (Tompkins et al., 2013). Furthermore, neurodegenerative disorders have been suggested as pathological outcomes of bTBI (Goldstein et al., 2012; Miller, 2012). While strong blasts may cause severe and acute brain injury or death, it is possible that exposure to mild forces result in delayed or more subtle effects. Thus, better understanding of the time- and force intensity-dependent factors that contribute to blast-induced pathophysiology is needed.

As animal models of bTBI have been developed over the past decade (Rubovitch et al., 2011b; Elder et al., 2012; Goldstein et al., 2012; Turner et al., 2013b), studies of blast exposure have descriptively characterized more overt pathological effects. Histopathology including profound neuronal injury and neuroinflammation has been demonstrated in rodent models of bTBI (de Lanerolle et al., 2011; Abdul-Muneer et al., 2013). Furthermore, bTBI has been suggested to result in suppression of synaptic plasticity in rat hippocampus (Miyazaki et al., 1992; Li et al., 2013a). Moreover, common biochemical and molecular mechanisms of brain injury have been demonstrated in a variety of bTBI models (Goldstein et al., 2012; Kochanek et al., 2013; Sosa et al., 2013; Turner et al., 2013b). Severe TBI elicits clear loss of neurological function, although the subsequent behavioral effects of blast-exposure and their persistence remain controversial (Cernak et al., 2001; Elder et al., 2012; Budde et al., 2013; Klemenhagen et al., 2013). While these more 
profound effects demonstrate the ability to accurately model severe bTBI, the threshold for pathogenesis in response to mild force remains poorly defined.

Here we investigate the histological, functional, and biochemical effects caused by exposure to mild blast. The results suggest that even mild blast forces may induce a variety of deleterious effects, which may not be reflected in behavioral or lower resolution imaging assessments. These results should inform the evaluations of patients exposed to mild or asymptomatic blast forces and, hopefully, contribute to facilitation of improved recovery from sub-concussive bTBI.

\section{METHODS}

Animals

All procedures involving animals were approved by the Institutional Animal Care and Use Committee of West Virginia University and were performed according to the principles of the Guide for the Care and Use of Laboratory Animals (IACUC protocol \# 13-0708). Male SpragueDawley rats $(n=74)$ were acquired from Hilltop Lab Animals (Hilltop Lab Animals, Inc., Scottsdale, PA). At the time of blast exposure the rats were 12 weeks old and weighed $\sim 300-350$ g. Animals were acclimated for 1 week prior to experimental use and were housed under 12-hour light/12-hour dark conditions with food and water available ad libitum.

\section{Blast Exposure}

Prior to blast exposure, animals were anesthetized with $4 \%$ isoflurane (Halocarbon, River Edge, NJ). The blast was delivered to the right side of the head with the animal's body oriented perpendicular to the blast tube, and with the peripheral organs protected by a polyvinyl chloride pipe shield. The animals were exposed to a mild blast $(0.005$ " membrane; incident peak 
overpressure of $\sim 15$ psi.), which was suggested to cause neural injury but no mortality in previous work (Turner et al., 2013b). Control subjects were placed in proximity to the blast paradigm but were not subjected to blast force. Immediately following blast exposure, animals were returned to a holding cage equipped with a homeothermic heating blanket equipped with a rectal thermometer to maintain body temperature at $37^{\circ} \mathrm{C}$. Once basic reflexes were restored, animals were returned to the home cage. For histological, neurophysiological, biochemical and behavioral paradigms, analyses were conducted after post-blast intervals of $6 \mathrm{~h}, 1,2,3$, or 7 days.

\section{Neuropathology}

Neuropathological studies were conducted at $1,2,3$, or $7 \mathrm{~d}(\mathrm{n}=6)$, with a control group. Animals were euthanized by $\mathrm{CO}_{2}$ asphyxiation and perfused transcardially with ice-cold $0.9 \%$ saline followed by $10 \%$ formalin for a total of $10 \mathrm{~min}$. The brains were then dissected and placed in fresh $10 \%$ formalin for $72 \mathrm{~h}$. Brains were then block-sectioned into 5 coronal slabs, paraffinembedded, and serially sectioned at $5 \mu \mathrm{m}$ at Bregma level of $2.20,1.00,-2.80,-7.30$ and -11.30 $\mathrm{mm}$. Standard protocols were utilized for staining with hematoxylin and eosin (H\&E) (Fischer et al., 2008), and Fluoro-Jade B (FJB, Millipore Corporation) (Schmued and Hopkins, 2000). For the immunohistochemical analysis, paraffin-embedded sections were labeled with SMI-31 to detect phosphorylated neurofilaments (1:500; Covance, Emeryville, CA), glial fibrillary acidic protein (GFAP) to detect astrocyte activation (1:1200; Chemicon) and ionized $\mathrm{Ca}^{2+}$ binding adapter molecule (Iba1) to detect microglial activation (1:1000; Wako) and visualized by immunoperoxidase method (Sinclair et al., 1981). Briefly, $5 \mu \mathrm{m}$ sections were deparaffinized, subjected to microwave antigen retrieval (citrate buffer, $\mathrm{pH}$ 6.0), permeabilized with $0.3 \%$ (vol/vol) Triton, quenched free of endogenous peroxidases, and blocked with a cocktail of normal goat serums $\left(2.5 \%(\mathrm{vol} / \mathrm{vol})\right.$ each) prior to overnight incubation of primary antibodies at $4{ }^{\circ} \mathrm{C}$. 
Bound primaries were detected by sequential incubation with biotinylated-secondary antisera and streptavidinperoxidase. Diaminobenzidine chromagen was used to detect immunoperoxidase signal (DAKO).

Neurophysiology

Neurophysiological studies were conducted on experimental and control rats 1,3 , or $7 \mathrm{~d}$ post-blast $(n=6)$. Following rapid decapitation and dissection, brains were placed into ice-cold solution $\left(75 \mathrm{mM}\right.$ sucrose, $87 \mathrm{mM} \mathrm{NaCl}, 2.5 \mathrm{mM} \mathrm{KCl}, 1.25 \mathrm{mM} \mathrm{NaH}_{2} \mathrm{PO}_{4}, 25 \mathrm{mM} \mathrm{NaHCO}, 7$ $\mathrm{mM} \mathrm{MgCl} 2,0.5 \mathrm{mM} \mathrm{CaCl}_{2}$ and $10 \mathrm{mM}$ glucose $)$, and transverse hippocampal slices $(350 \mu \mathrm{m})$ were prepared using a vibratome (Leica Microsystems Inc., VT1000S). Slices recovered in oxygenated Kreb's buffer (125 mM NaCl, $2.5 \mathrm{mM} \mathrm{KCl,} 1.25 \mathrm{mM} \mathrm{NaH}_{2} \mathrm{PO} 4,25 \mathrm{mM} \mathrm{NaHCO}_{3}, 1.1 \mathrm{mM} \mathrm{MgCl}_{2}$, $2 \mathrm{mM} \mathrm{CaCl}_{2}$ and $25 \mathrm{mM}$ glucose) and maintained at $30^{\circ} \mathrm{C}$ for $30 \mathrm{~min}$ before recording. A Multiclamp 700A amplifier with a Digidata 1322 and pClamp 10 software (Axon, Molecular devices, LLC) was used to for the electrophysiological recordings. Slices for recordings were placed into a perfusion chamber on the upright microscope stage (Axioscop 2, Carl Zeiss, Inc). The perfusion bath was maintained at $30^{\circ} \mathrm{C}$ during the recordings (TC-324B Automatic Temperature Controller, Warner Instruments Corporation). Field excitatory postsynaptic potentials (fEPSP) from CA1 were evoked by square current pulses $(0.1 \mathrm{~ms})$ at $0.033 \mathrm{~Hz}$ with a bipolar stimulation electrode (FHC, Bowdoinham, ME) placed at the Schaffer collaterals ( 250$300 \mu \mathrm{m})$ from the recording electrode. Results were obtained using a stimulus intensity to induce $50 \%$ of the maximal fEPSP slope, with the same intensity used to explore the paired pulse ratio (PPR) at different intervals. The intensity was established through the input-output curve, the maximal stimulation was considered when a population spike appeared in the fEPSP. A stable baseline was recorded for at least 15 min prior to high frequency stimulation (HFS, 4 trains, 100 
$\mathrm{Hz}, 1 \mathrm{~s}$ duration, separated by $20 \mathrm{~s}$ ). Long-term potentiation (LTP) was assessed for at least $60 \mathrm{~min}$ after HFS. The time-course showing baselines and LTP is expressed as a percent of change from the baseline fEPSP slope. The PPF plot was constructed by dividing the second fEPSP slope over the first (fEPSP2/fEPSP1). The input-output curve was constructed by normalizing recordings for each slice, assigning the maximal fiber volley amplitude (obtained with the maximal stimulation) to the value of 1.0, and the plots were derived for the respective fEPSP slopes. All recordings were taken in the absence of any drug. Data were analyzed with Clampfit 10 software (Axon, Molecular devices, LLC). Prism 6 (GraphPad Software, Inc.) was used to make graphs and statistical analysis.

\section{Immunoblotting}

Brain dissection, tissue removal and lysate preparation, and immunoblotting were performed as previously described (Sahin et al., 2004; Plattner et al., 2006). Rats ( $\leq 6$ ) for immunoblotting were sacrificed at $6 \mathrm{~h}$, and 1,2,3 and $7 \mathrm{~d}$ post-blast exposure. Cell lysates from hippocampal dissections were prepared and equal amounts of lysates were run on $7 \%$ acrylamide gels or 10-20\% gradient acrylamide gels. The blots were probed with the following antibodies: p35/25 (Cell Signaling Technology; C64B10), $\alpha$-Fodrin (cleaved spectrin) (Enzo Life Sciences;

BML FG6090), pTau $^{\mathrm{T} 205}$ (Sigma; T 6694), total Tau (DAKO). Signal was detected with HRPconjugated secondary antibodies and enhanced chemiluminescence (GE Healthcare, Piscataway, $\mathrm{NJ})$. Images were analyzed using densitometry with background subtraction and ImageJ software (NIH, Bethesda, MD). The resulting bands were normalized to GAPDH (10-20\% gels), B-tubulin (7\% gels), or total Tau.

\section{Locomotion}

All behavior was carried out with the experimenter blind to treatment groups. Locomotion trials were conducted at $48 \mathrm{~h}$ post blast. Rats $(\mathrm{n}=7)$ were left in their home cage surrounded by a 
PAS-Home Cage locomotor recording apparatus (San Diego Instruments, CA). The rats were left to explore for $24 \mathrm{~h}$ and ambulatory, fine and total beam breaks were collected with Vision PASF Software (San Diego Instruments). Ambulatory beam breaks were counted as the simultaneous breaking of multiple beams within $0.2 \mathrm{~s}$, and Fine beam breaks were counted as single beam breaks at intervals greater than $0.2 \mathrm{~s}$. The two forms of beam breaks were combined to compare total beam breaks. The locomotion was subdivided into 24 bins of $1 \mathrm{~h}$ each.

\section{Fear Conditioning}

The fear conditioning behavioral paradigm used here was essentially as previously described (Plattner, 2014). Rats $(n=7)$ were left to explore the conditioning box (Med Associates) for $2 \mathrm{~min}$, followed by a $30 \mathrm{~s}$ period with tone representation $(90 \mathrm{~dB}, 10 \mathrm{kHz})$, which co-terminated with a mild foot shock ( $2 \mathrm{~s}, 0.7 \mathrm{~mA}$, constant current). Rats remained in the chamber 2 min after shocking before returning to their home cage. Conditioning boxes were thoroughly cleaned after each animal. To test context-dependent fear memory, rats were reintroduced $24 \mathrm{~h}$ post-shock into the conditioning box for 5 min. Freezing responses (motionless except respirations) were recorded using a $5 \mathrm{~s}$ interval time-sampling method. Control animals were subject to isoflurane exposure (4\%) on the same day as the bTBI rats.

\section{Statistical Analysis}

Data were analyzed using GraphPad Prism 6 (GraphPad Software, Inc., La Jolla, CA). Oneway ANOVA with post-hoc Newman-Keuls multiple comparison tests were used to compare across groups for the neurophysiological data. Student's $t$-tests were used to compare behavioral and immunoblot data. A $p<0.05$ was considered statistically significant for all data analyzed.

\section{RESULTS}


To study the threshold effects of bTBI it is important to define the relationship between force intensity and injury severity in a general sense. Previously we characterized this relationship with regard to general neuropathology and function using a scaled blast model that we developed (Turner et al., 2013b). This study defined incident peak overpressure of 15 psi exposure as the threshold for induction of neuropathological effects, consistent with those reported in other studies (Goldstein et al., 2012; Mez et al., 2013; Sosa et al., 2013). Based on these findings we chose to expose young adult male rats to a single threshold shock wave (Figure 3.1A). To assess effects over relatively acute periods following blast exposure, delays including $6 \mathrm{~h}$, and 1,2,3 and $7 \mathrm{~d}$ were chosen (Figure 3.1B) for histology, neurophysiology, biochemical, and behavioral analyses.

\section{Neuropathological effects of mild bTBI}

Gross examination of postmortem brains from each time point following blast exposure revealed no macroscopic evidence of contusion, necrosis, hematoma, hemorrhage, or focal tissue damage (Figure 3.2A-C), confirming the force intensity used as mild or threshold. However detailed microscopic examination showed a variety of notable deleterious effects in blast-exposed rats. Small hemorrhagic foci, which showed the presence of the intraparenchymal red blood cells (Figure 3.2D) and robust reactive astrogliosis (Figure 3.2E), were observed in the left corpus collosum at $48 \mathrm{~h}$ post-blast and in the striatum at $72 \mathrm{~h}$ post-blast. Furthermore, the extravasation of blood plasma was confirmed in a small number of venule- (Figure 3.2F) and arteriole-like (Figure 3.2G) microvessels of the cerebral cortex or striatum at all time points post-blast. These observations are consistent with a growing perception of the microvasculature as an important target of bTBI research (Sajja et al., 2013).

Diffuse axonal injury, one of the primary neuropathological characteristics of TBI, was observed at all time points post-blast. Phosphorylated neurofilament immunostaining was 
enhanced in the deep layer of cerebral cortex and lateral septal nucleus (Figure 3.2H), as well as in the ventrolateral part of thalamus (Figure 3.2J), compared to control subjects (Figure 3.2I, K). These effects were accompanied by beaded or irregularly swollen dystrophic axons (Figure 3.2L) and axonal bulbs (Figure 3.2M). These malformations in axonal architecture denote potential compromise in neuronal circuitry. Furthermore these effects may denote neurofilament compaction or diffuse axonal injury which has been implicated in TBI neuropathogenesis (Blennow et al., 2012).

As was observed above, the effects of blast on fine microvasculature and axonal structure were accompanied by elevated reactive astrocytes and microglia throughout the cerebrum, especially in the striatum, cortex, amygdala and hippocampus, as well as in the thalamus. Reactive astrocytes were observed in a greater abundance in the cerebral cortex $72 \mathrm{~h}$ post-blast (Figure $3.2 \mathrm{~N}$ ), compared to control subjects (Figure 3.2O). Reactive microglia were also observed in greater abundance in the striatum $72 \mathrm{~h}$ post-blast (Figure 3.2P), compared to control subjects (Figure 3.2Q). In contrast, brainstem and cerebellum were relatively devoid of neuroinflammation. The hippocampus is often the target of neurodegenerative pathology (Nagy et al., 1997), and neurophysiological function of hippocampal circuitry is often related to cognitive performance (Wiescholleck and Manahan-Vaughan, 2013b, a). Therefore, to better understand the effects of mild blast exposure, and given the overall pathology observed in mild blast-exposed rats, hippocampus pathology was examined. Postmortem coronal sections of hippocampus from each time point post-blast were well preserved (Figure 3.3A). Furthermore, H\&E staining showed no signs of typical degenerating or ischemic neurons in the hippocampus following blast injury (Figure 3.3B). FJB staining also showed no signs of neurodegeneration in the hippocampus of 
bTBI rats (Figure 3.3C). However, brains from bTBI rats showed other evidence of microscopic neuropathology in the hippocampus.

For example, the hippocampal subfield CA3 exhibited intense immunoreactivity for phosphorylated neurofilaments. SMI-31-immunoreactive. Staining displayed an accumulation of phosphorylated neurofilaments in the cytoplasm of several pyramidal neurons (Figure 3.3D). These effects were completely absent in control subjects (Figure 3.3E). A small number of mild swollen dystrophic axons were observed in the CA1 stratum pyramidale (Figure 3.3F) or radiatum at all time points post-blast. One axonal bulb was seen in the hippocampal hilus at $7 \mathrm{~d}$ post-blast (Figure 3.3G). Brains subjected to bTBI also demonstrated obvious reactive astrogliosis and microgliosis throughout the hippocampus. Reactive astrocytes were observed in a greater abundance in the CA1 field $72 \mathrm{~h}$ post-blast (Figure 3.3H), compared to control subjects (Figure 3.3I). Reactive microglia were also observed in greater abundance in the CA1 subfield $72 \mathrm{~h}$ postblast (Figure 3.3J), compared to control subjects (Figure 3.3K). Thus neuropathological effects observed in the hippocampus were consistent with the neuropathology observed in other brain regions.

\section{Neurophysiological effects of mild bTBI}

To understand the physiological consequences of the neuropathology resulting from mild blast-exposure, neurophysiological studies were conducted in acutely prepared hippocampal brain slices. To assess hippocampal circuitry, functional LTP was assessed in the Schaffer collateralCA1 circuitry. HFS induced robust LTP in control and bTBI rats, with tetanic stimulus resulting in a $166 \pm 11 \%$ potentiation of fEPSP slope compared to baseline in control subjects at $60 \mathrm{~min}$ post-stimulus $(p<0.03$, paired Student's $t$ test). Despite the neuropathology revealed in our histological assessment, mild blast exposure had no significant effect on LTP induction after 60 
min at any of the post-blast delay time points examined (Figure 3.4A). In contrast, assessment of synaptic responses during the first 2 min following HFS revealed that post-tetanic potentiation (PTP) was attenuated in rats after a 7-day post-blast delay $(p<0.05$, ANOVA, Newman-Keuls post hoc) (Figure 3.4B; top).

In support of this observation, short-term plasticity as a function of paired pulse ratio (PPR) was also examined. A significant reduction in PPR was observed during the first 2 min following paired pulsation in the control subjects ( $p<0.03$, Mann-Whitney test) (Figure 3.4B; bottom). However $7 \mathrm{~d}$ post-blast subjects did not exhibit this form of plasticity. Thus blast exposure results in a reduced probability of neurotransmitter release from presynaptic terminals compared to control subjects. This reduction in synaptic response, in the absence of effects on LTP, suggests that mild blast exposure selectively affects short term plasticity at high frequency of stimulation and is consistent with disruption of axonal conduction, as has been proposed as an outcome of blast injury (Baalman et al., 2013).

As another approach to assess short-term plasticity, paired pulse ratio (PPR) of synaptically induced fEPSPs was also examined. A significant reduction in PPR was observed during the first 2 min following paired pulsation in the control subjects $(p<0.03$, Mann-Whitney test) (Figure 3.4B; bottom). However $7 \mathrm{~d}$ post-blast subjects did not exhibit this form of plasticity. Thus blast exposure results in a reduced probability of neurotransmitter release from presynaptic terminals compared to control subjects.

To assess hippocampal synaptic excitability, field potential recordings were used to derive input-output curves (Figure 3.4C). Rats that underwent a $7 \mathrm{~d}$ post-blast delay exhibited a significant decrease in maximal response $(p<0.01$, nonlinear regression using a polynomial quadratic function; $p<0.03$, maximal stimulation in control vs. $7 \mathrm{~d}$ post-blast, Mann-Whitney 
test), indicating a reduction in synaptic response intensity. To better understand this deficit, paired pulse facilitation (PPF), was assessed at different intervals (Figure 3.4D). Interestingly, no difference in PPF was observed across a range of inter-pulse intervals for rats subjected to different post-blast delay periods compared to control subjects. These results indicate that while mild blast exposure resulted in deficits of short-term plasticity and synaptic responses, they caused no important changes to basic synaptic transmission at presynaptic terminals.

Overall, these results show subtle deficits in the neurophysiological function of hippocampal circuitry in response to mild blast exposure. While long-term plasticity was not significantly altered, the changes in short-term plasticity and synaptic excitability are consistent with other reports of neurophysiological deficits following traumatic brain injury (Miyazaki et al., 1992; Reeves et al., 2005; Colley et al., 2010), or more specifically, blast exposure (Park et al., 2013).

\section{Excitotoxic effects of mild bTBI}

TBI causes massive neuronal depolarization resulting in large influx of ions. In response to activation of voltage-gated $\mathrm{Ca}^{2+}$ channels, high levels of glutamate are released, triggering excitotoxicity. Swelling of neurons, oxidative stress, and free radical production, all affect neuronal viability, contribute to neuronal death, and are associated with TBI pathology (Cernak and Noble-Haeusslein, 2010). Following initial trauma, a delayed and spreading process of injury occurs. At the subcellular level, mitochondrial dysfunction and disruption in $\mathrm{Ca}^{2+}$ homeostasis are hallmarks of TBI (Wang et al., 2006). Over-activated cellular proteases such as calpain may contribute to injury progression (Werner and Engelhard, 2007). The effects of mild bTBI on these parameters were examined in hippocampal lysates from blast exposed rat brain by quantitative immunoblotting. First, levels of cleaved spectrin were assessed as a marker of calpain activity 
(Figure 3.5A). Calpain levels were significantly elevated at 3 and $7 \mathrm{~d}$ post-blast in comparison to controls.

Another important calpain substrate is $\mathrm{p} 35$, the activating cofactor for the protein kinase Cdk5 (Angelo et al., 2006). Calpain-dependent cleavage of p35 produces the truncated protein p25. Cdk5/25 engenders aberrant activity and contributes to neuronal cell death (Hisanaga and Endo, 2010). Consistent with an increase in calpain activity, as indicated by spectrin cleavage, p25 levels were significantly elevated at 3 and $7 \mathrm{~d}$ following mild bTBI (Figure 3.5B).

The hyperphosphorylation of the microtubule-associated protein, tau, and the resulting formation of neurofibrillary tangles may characterize TBI (Smith et al., 2013). Furthermore, Thr205 tau is a substrate of Cdk5/p25 (Maccioni et al., 2001). Interestingly, mild bTBI induced acute elevations in phospho-Thr205 tau with significant increases detected at 6, 24, and $48 \mathrm{~h}$ after mild bTBI (Figure 3.5C). Moreover, by $72 \mathrm{~h}$, the levels were significantly below that of control subjects. Taken together these results demonstrate that effectors of excitotoxicity are invoked by mild blast exposure, and may underlie the pathological and neurophysiological deficits detected.

\section{Behavioral effects of mild bTBI}

To further assess the functional outcomes of mild blast injury we employed a wellestablished fear conditioning protocol (Plattner, 2014). In this behavioral paradigm, normal subjects can predict aversive outcomes through association with a neutral context in which an aversive stimulus was previously presented (Maren, 2001). The conditional response to the neutral context (fear conditioning box) has long been associated with hippocampal function and injury (Corcoran and Maren, 2001). Importantly, no changes in contextual fear response were observed 3 and $7 \mathrm{~d}$ following mild blast exposure (Figure 3.6A). Furthermore, mild blast-exposed rats exhibited no effects on locomotion in fine (Figure 3.6B), ambulatory (Figure 3.6C) and total 
(Figure 3.6D) activity over a $24 \mathrm{~h}$ period in comparison to control subjects. Thus, the neuropathological, neurophysiological, and biochemical effects of mild blast exposure did not manifest in behavioral changes at the relatively early post-blast time points examined.

\section{DISCUSSION}

Here we used a bench-top model of bTBI that we developed (Turner et al., 2013b) to explore the threshold of blast-exposure-induced brain injury. While rodent models of bTBI have been used to demonstrate neuronal injury (Goldstein et al., 2012) and death (Turner et al., 2013b), here we show that mild blast exposure elicited no macroscopic tissue damage or detectible behavioral effects. However, close examination revealed axonal injury accompanied by damage to microvasculature, with the blood brain barrier compromised at sparse foci throughout the brain. These effects are consistent with the loss of hemodynamics, edema, and hemorrhage that characterize more severe TBI. Interestingly, microvascular defects may characterize presymptomatic phases of Alzheimer's disease, suggesting a possible link between these effects and increased incidence of neurological disease in blast exposed individuals (Dennis et al., 2009).

Numerous studies have demonstrated that neurotrauma can affect synaptic plasticity (Bach-y-Rita, 2003; Page and Yablon, 2003; Griesbach et al., 2004), including in developing rat brains (Li et al., 2013a). Suppression of LTP has been demonstrated in more severe models of bTBI (Miyazaki et al., 1992; De Beaumont et al., 2012). Other traumatic brain injury models have shown decreased hippocampal LTP levels with attenuated cognitive function (Titus et al., 2013). In contrast, the mild blast forces used here had no detectable effect on LTP. Furthermore, the overall functional synaptic properties were not dramatically affected by blast exposure. However, mild blast exposure caused deficiencies in PTP following HFS and synaptic excitability. PTP may 
be mediated by long-lasting increase in presynaptic $\mathrm{Ca}^{2+}$ concentrations that elevate the release probability of the presynaptic readily releasable pool of synaptic vesicles (Habets and Borst, 2005). The deficiencies in this form of short-term plasticity and synaptic responsiveness may be causally linked to the malformations of axonal processes observed histologically. While the effects observed may be only weakly represented in evaluations of working memory or cognitive performance, future studies, using repetitive mild blast exposures for example, may reveal latent consequences on synaptic long-term plasticity or learning and memory.

Loss of microvascular integrity and blood brain barrier compromise are often related to brain injuries involving excitotoxicity. Activation of $\mathrm{Ca}^{2+}$-dependent proteases such as calpain is a predicted outcome of membrane depolarization and loss of $\mathrm{Ca}^{2+}$ homeostasis. Spectrin is involved in actin binding and maintaining the shape of synapses. Its regulation of cytoskeletal dynamics contributes to plasticity (Greer et al., 2013) and may be required for learning (Andres et al., 2013). Furthermore, calpain-mediated degradation of neurofilament proteins has been implicated in TBI (Blennow et al., 2012). Thus calpain activation and cleavage of spectrin is consistent with deficits in axonal architecture and disruption of synaptic plasticity (Ma, 2013). Indeed, rats exposed to bTBI can exhibit shortened axon initial segments, suggesting such subcellular changes (Baalman et al., 2013). Moreover, spectrin cleavage in the corpus callosum after bTBI has been suggested to attenuate overall electrophysiological responses (Park et al., 2013) and neuronal death (Schafer et al., 2009).

Calpain-dependent conversion of p35 to p25 results in dysregulation of Cdk5, causing relocation of the protein kinase and redirection towards aberrant substrates that mediate neuronal injury (Plattner et al., 2006). While many of the substrates by which aberrant Cdk5 mediates neurotoxicity remain to be delineated, one example is phospho-Thr205 tau, which increased at the 
onset of p25 generation in response to mild blast exposure. The long-term effects of bTBI include tau hyperphosphorylation and neurofibrillary tangle accumulation (Goldstein et al., 2012; Weiner, 2013). Thus exposure to moderate blast forces may initiate subtle but still meaningful neuropathological processes.

Preclinical studies of the effects of blast exposure in the past decade have focused predominantly on the hippocampus as an area critical to learning and memory and neuropsychiatric sequela (Taber et al., 2006; Cernak and Noble-Haeusslein, 2010; Budde et al., 2013; Huber et al., 2013; Scheper and Hoozemans, 2013; Tweedie et al., 2013). Findings suggest that the exposure to bTBI triggers early hippocampal cell damage which increases the incidence of depression and anxiety related behaviors (Kamnaksh et al., 2012). Effects in other brain regions are also being explored with regard to excitotoxic effects or contributions to the development of mood disorders (Combes, 2013; Sajja et al., 2013). Taken together, the results presented here demonstrate that mild blast exposure causes a panoply of deleterious neurological effects in the absence of major behavioral deficits. It remains to be determined if mild blast exposures and the subclinical effects detected here will develop into neuropsychiatric conditions with adequate periods of incubation. 

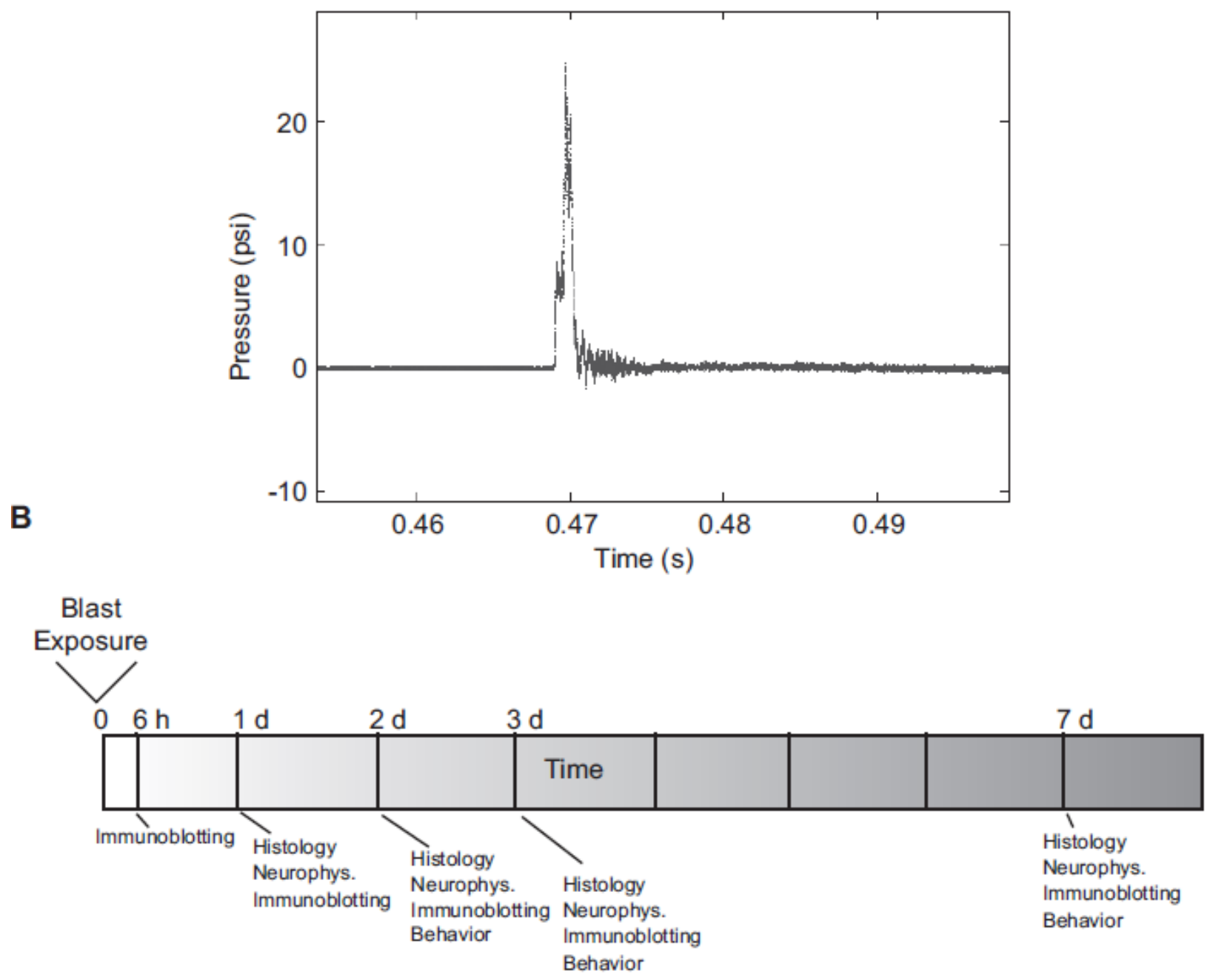

Figure 3.1. Blast pressure recording and experimental design. A. Recording of Friedlander short-duration shock wave to which rats in this study were exposed. B. Schematic portraying experimental design which includes time points for animal sacrifice for the neuropathology, neurophysiology, and immunoblotting assays, or days after blast exposure for behavioral testing. Control animals were not exposed to blast pressure. 


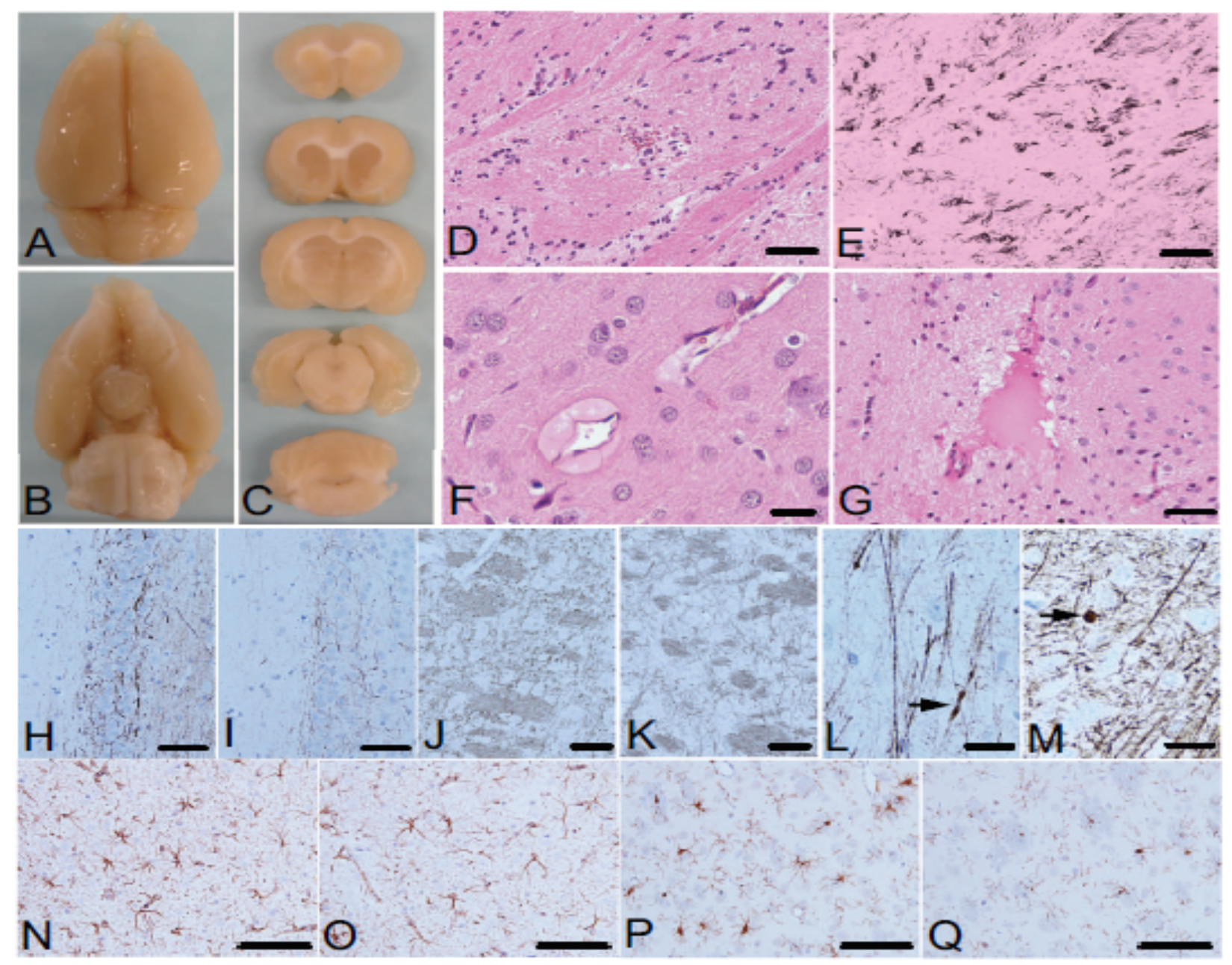

Figure 3.2. Mild blast exposure had no effect at the macroscopic level, but causes microvascular injury, axonal damage, and neuroinflammation. A-C. Absence of macroscopic tissue damage $7 \mathrm{~d}$ post-blast. Dorsal (A), ventral (B) and anterior-posterior coronal $4 \mathrm{~mm}$ brain section $(\mathbf{C})$ views are shown. D. Microvascular injury and dysfunction $72 \mathrm{~h}$ postblast indicated by small hemorrhagic focus (center) in the corpus collosum. E. Robust reactive astrogliosis in the same area as in D. F. Extravasation of blood plasma in a venule-like microvessel in the left deep layer of cerebral cortex $72 \mathrm{~h}$ post-blast. G. Extravasation of blood plasma in an arteriole-like microvessel in the right nucleus accumbens $48 \mathrm{~h}$ post-blast. $\mathbf{H}$ \& I. Axonal injury indicated by increased phosphorylated neurofilament immunostaining in the left lateral septal nucleus $7 \mathrm{~d}$ post-blast $(\mathbf{H})$; compared to control (I). J \& K. Axonal injury also indicated in thalamus $72 \mathrm{~h}$ post-blast $(\mathbf{J})$; compared to control $(\mathbf{K})$. L. Beaded, irregularly swollen dystrophic axons (arrow) in the left lateral septal nucleus $24 \mathrm{~h}$ post-blast. M. Axonal bulb (arrow) at the interface between cerebral cortex and corpus callosum $72 \mathrm{~h}$ post-blast. $\mathbf{N} \boldsymbol{\&}$ O. Neuroinflammation indicated by increased astrocyte activation in the deep layer of cerebral cortex $72 \mathrm{~h}$ post-blast $(\mathrm{N})$; compared to the same area from control $(\mathrm{O})$. $\mathbf{P} \& \mathbf{Q}$.

Neuroinflammation also indicated by increased number of reactive microglia in striatum $72 \mathrm{~h}$ post-blast (P); compared to the same area from control (Q). H\&E staining (D,F,G). SMI 31 immunostaining (H-M). GFAP immunostaining (E,N,O). Iba1 immunostaining (P,Q). Scale bars: $50 \mu \mathrm{m}$ (D,E,G,H-K,N-Q); $20 \mu \mathrm{m}(\mathbf{F}, \mathbf{L}, \mathbf{M})$. 


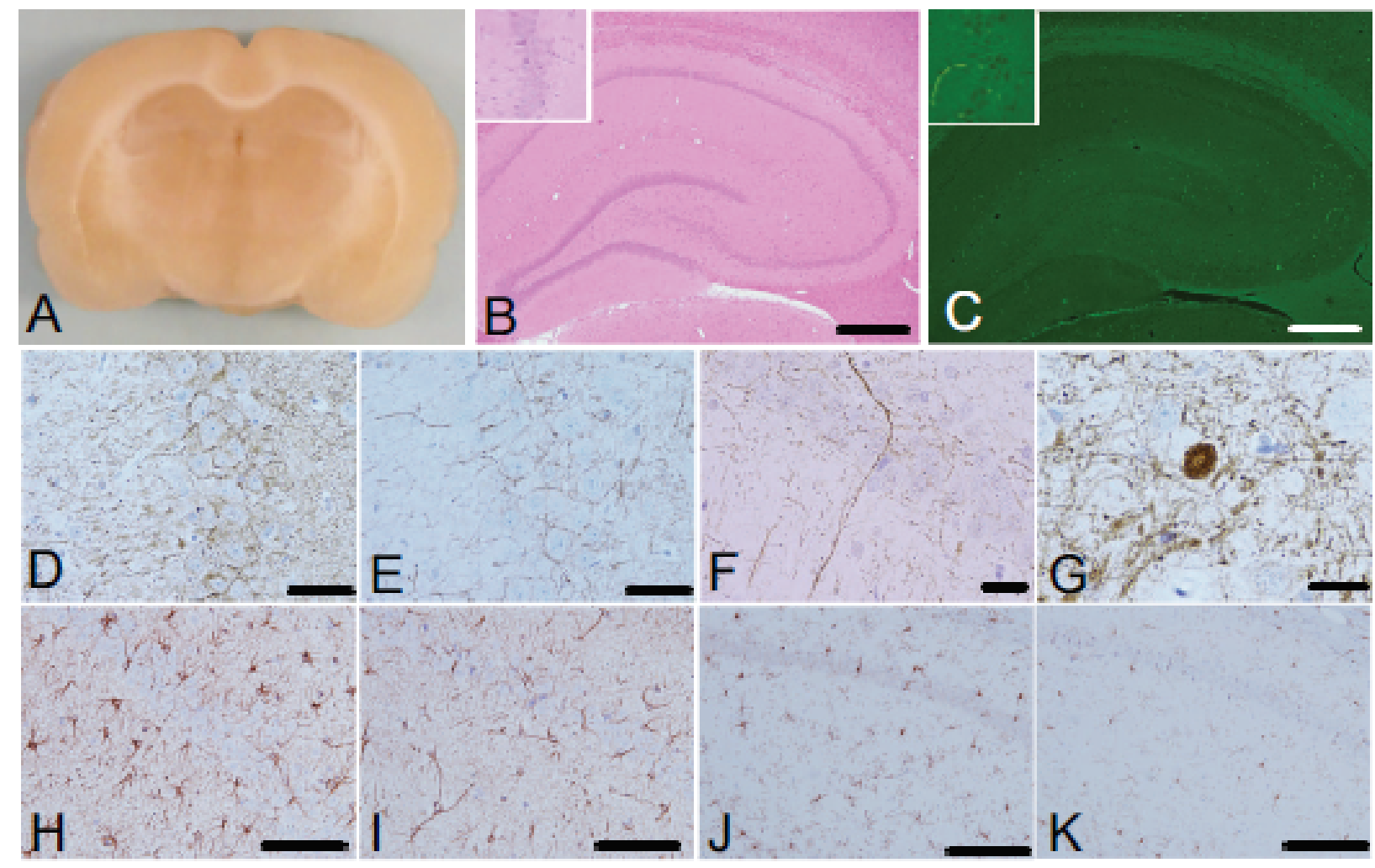

Figure 3.3. Mild blast exposure does not induce macroscopic changes to the hippocampus, but does induce microscopic hippocampal pathology as indicated by axonal damage and neuroinflammation. A. Absence of macroscopic hippocampal tissue damage $7 \mathrm{~d}$ post-blast. B. Absence of overt neuronal injury in hippocampus $7 \mathrm{~d}$ post-blast. Insert shows no significant pathology for CA3 field. C. Absence of FJB-positive neurons in the hippocampus $7 \mathrm{~d}$ post-blast. Insert shows no degenerating neurons for CA3 field. D \& E. Increased phosphorylated neurofilament immunostaining in the hippocampal CA3 field $48 \mathrm{~h}$ post-blast (D); compared to the same area from control rats (E). F. Mild swollen atrophic axon in hippocampal CA1 stratum pyramidale $48 \mathrm{~h}$ post-blast. G. Axonal bulb in the hippocampal hilus $7 \mathrm{~d}$ post-blast (arrow). H \& I. Increased astrogliosis in the hippocampal CA1 field $72 \mathrm{~h}$ post-blast $(\mathbf{H})$; compared to the same area from control (I). J \& K. Increased number of microglia in CA1 $72 \mathrm{~h}$ post-blast (J); compared to the same area from control (K). H\&E staining (B). FJB staining (C). SMI 31 immunostaining (D-G). GFAP immunostaining (H \& I). Iba1 immunostaining (J \& K). Scale bars: $50 \mu \mathrm{m}(\mathbf{B}-\mathbf{E}, \mathbf{H}, \mathbf{I}) ; 20 \mu \mathrm{m}(\mathbf{F}, \mathbf{G}) ; 100 \mu \mathrm{m}(\mathbf{J}, \mathbf{K})$. 

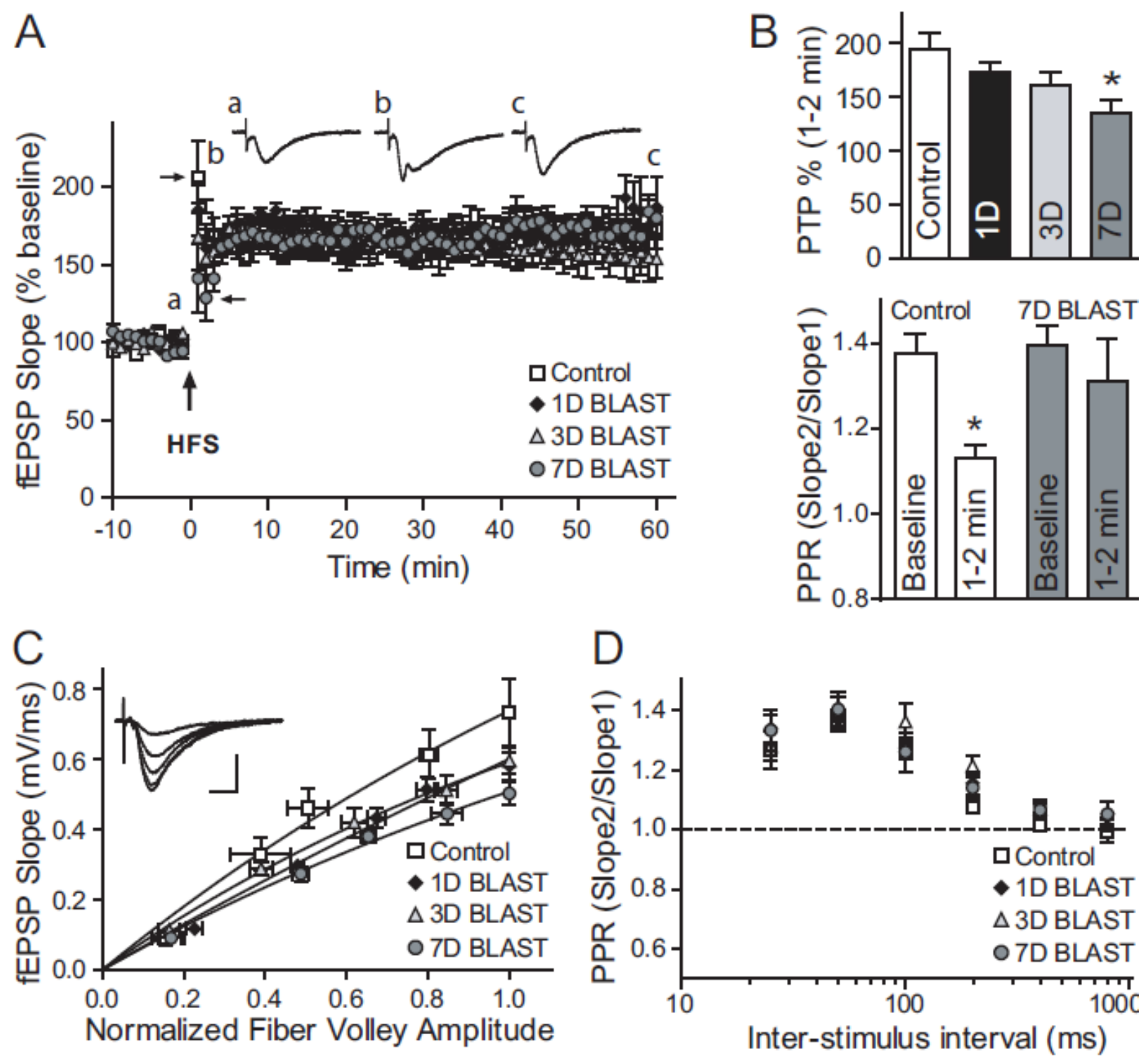

Figure 3.4. Mild blast exposure causes neurophysiological deficits in hippocampus. A. Assessment of blast effects on LTP. B. Time-course shows plot of fEPSP slopes (percent baseline). Each point represents average from two traces. Insets show representative traces from control recordings (a: baseline, b: PTP, c: LTP). Horizontal arrows show PTP difference between control and $7 \mathrm{~d}$ post-blast. Deficits in short-term plasticity following HFS are induced by mild blast exposure. C. The upper panel summarizes fEPSP slopes during the first 2 min post-HFS. The lower panel shows effects on PPR during the first 2 min post-HFS at $7 \mathrm{~d}$ post-blast. Input-output curves measuring fEPSP slopes against normalized fiber volley amplitudes. A significant reduction in maximal response was observed $7 \mathrm{~d}$ post-blast. Inset shows representative traces from control recordings at different stimulation intensities. D. Paired pulse ratio at different intervals shows no change in short-term synaptic plasticity following blast. (Mean $\pm \mathrm{SEM} ; \mathrm{n}=6,{ }^{*} p<0.05$, ANOVA, Newman-Keuls post hoc). 

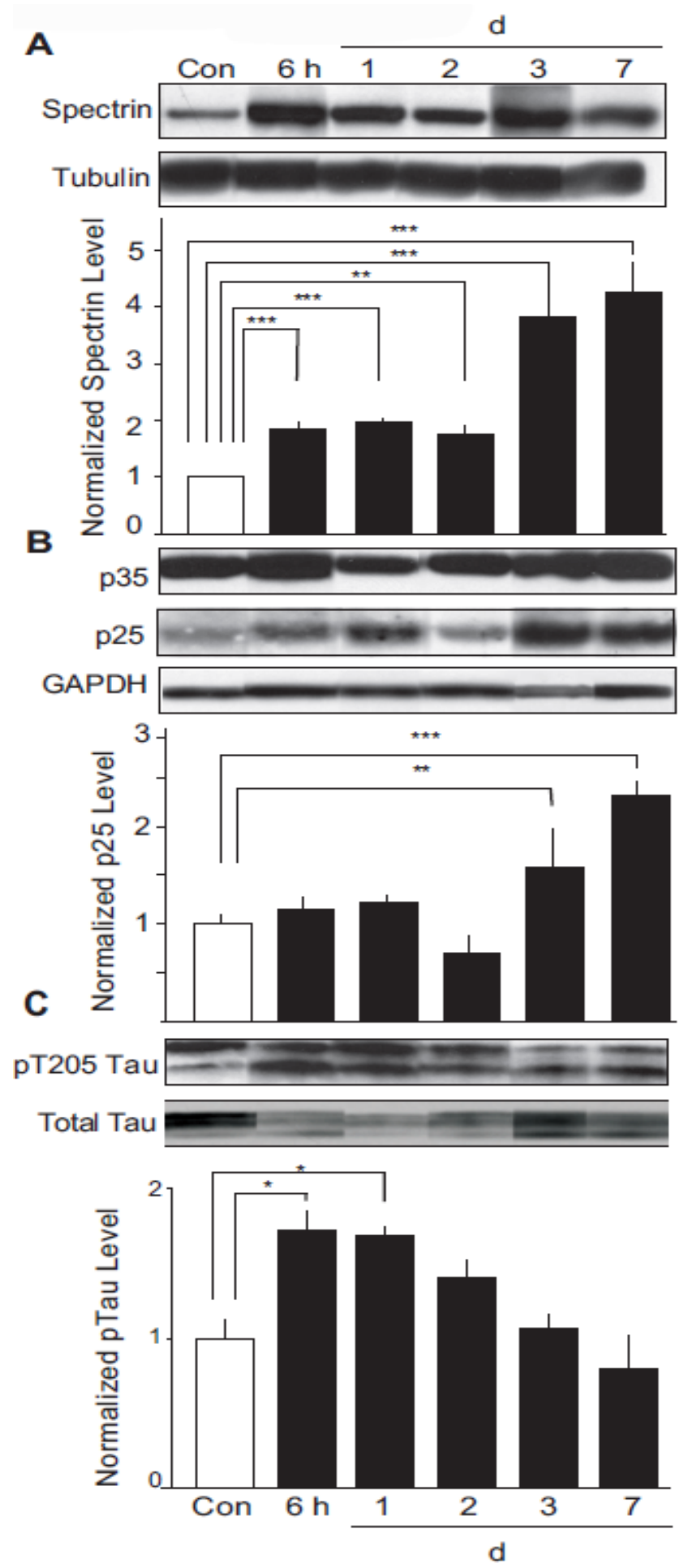

Figure 3.5. Mild blast exposure invokes markers of excitotoxicity and neuronal injury in hippocampus. A-C. Quantitative immunoblot analyses of hippocampal lysates from control and blast-exposed rats at the indicated post-blast interval are shown for the calpain-cleaved form of spectrin (A), p25, the calpain-cleaved form of the Cdk5 activator, p35 (B), and phospho-Ser205 tau (C). Data represent means \pm s.e.m.; $\mathrm{n} \leq 6$; ${ }^{*} p<0.05,{ }^{*} p<0.01,{ }^{* *} p<0.005$; Student's $t$ test. 

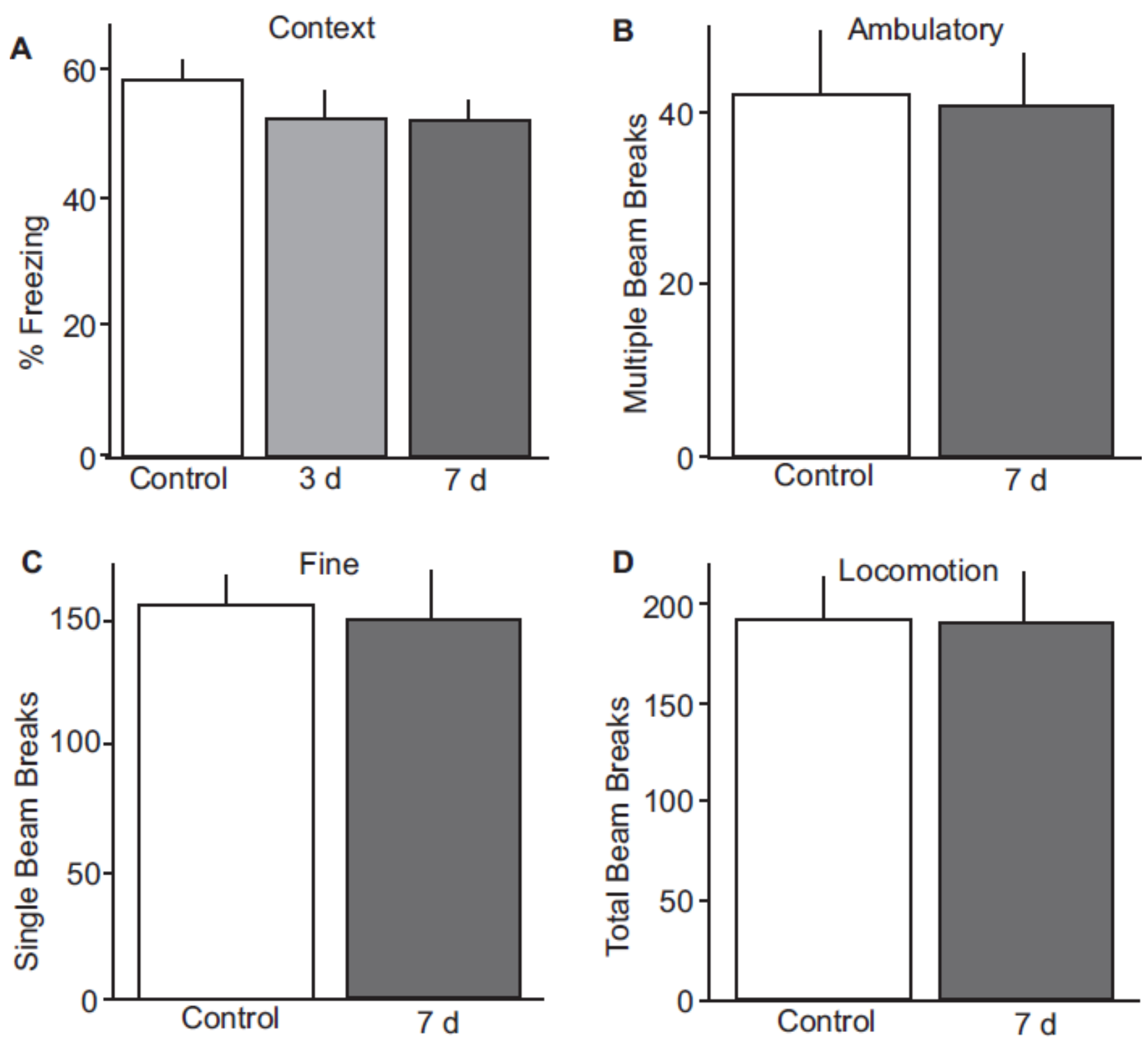

Figure 3.6. Mild blast exposure has no effect on contextual fear conditioning or locomotor activity. A. Contextual fear conditioning trials revealed no significant difference in the percent freezing at 3 and $7 \mathrm{~d}$ post-blast compared to control subjects. B-D. Analysis of $24 \mathrm{~h}$ home cage locomotion revealed that mild blast exposure showed no effect on ambulatory $(\mathbf{B})$, fine $(\mathbf{C})$, or total locomotion (D) compared to control subjects. Data represent means \pm s.e.m.; $\mathrm{n}=7$; Student's $t$-test versus control. 


\title{
Chapter Four
}

\begin{abstract}
Altering endoplasmic reticulum stress in a model of blast-induced traumatic brain injury controls cellular fate and ameliorates neuropsychiatric symptoms
\end{abstract}

This work is published in Front Cell Neurosci. 2014 Dec 10;8:421. doi: 10.3389/fncel.2014.00421. eCollection 2014. PMID: 25540611 Logsdon AF, Turner RC, Lucke-Wold BP, Robson MJ, Naser ZJ, Smith KE, Matsumoto RR, Huber JD, Rosen CL. 


\section{ABSTRACT}

Neuronal injury following blast-induced traumatic brain injury increases the risk for neuropsychiatric disorders, yet the pathophysiology remains poorly understood. Blood-brainbarrier disruption, endoplasmic reticulum stress, and apoptosis have all been implicated in rodent models of neural injury. Microvessel compromise is a primary effect of neural injury and is postulated to cause subcellular secondary effects apoptosis. What remains unclear is how these secondary effects progress to personality disorders in humans exposed to head trauma. To investigate this we exposed male rats to a clinically-relevant blast model we have recently developed. The study examined initial vascular disruption using Evan's blue, secondary injury mechanisms, apoptosis, and impulsive-like behavior measured with elevated plus maze. Evan's blue extravasation was observed immediately following blast injury, and persisted for at least $6 \mathrm{~h}$. Data showed increased mRNA abundance of stress response genes at $3 \mathrm{~h}$, with subsequent increases in cellular stress markers C/EBP homologous protein, and growth arrest and DNA damage-inducible protein 34, at $24 \mathrm{~h}$. Caspase-12 and Caspase-3 were both cleaved at $24 \mathrm{~h}$ following blast injury. The endoplasmic reticulum stress inhibitor, salubrinal, was administered $(1 \mathrm{mg} / \mathrm{kg}$ i.p.) to investigate its' effects on neuronal injury and impulsive-like behavior associated with neurotrauma. Salubrinal reduced protein expression of cellular stress markers, and diminished Caspase-3 cleavage, suggesting apoptosis attenuation. Interestingly, salubrinal also ameliorated impulsive-like behavior indicative of head trauma. These results suggest salubrinal to play a role in the regulation of apoptosis and the pathology of chronic disease. These observations provide evidence that endoplasmic reticulum stress is a promising molecular target for the attenuation of neuronal injury after neurotrauma. 


\section{INTRODUCTION}

Blast-induced traumatic brain injury (bTBI) has been described as the 'hallmark injury' of recent wars in Iraq and Afghanistan (Goldstein et al., 2012). The Defense and Veterans Brain Injury Center estimates that approximately 270,000 blast exposures have occurred over the past decade (Farrell-Carnahan et al., 2013). Many blast exposures cause concussive or sub-concussive brain damage and are associated with the shearing of axons (Rosenfeld and Ford, 2010) and the compromise of brain micro-vessels (Chen et al., 2013b). Often these injuries go undetected in soldiers and civilians due to poor understanding of the underlying mechanisms of blast injury and the diagnostic limitations preventing the detection of pathophysiologic changes in living patients (Stern et al., 2011).

Blast exposure can cause blood brain barrier (BBB) dysfunction (Abdul-Muneer et al., 2013; Chen et al., 2013b) and induce short-term inflammatory cascades that promote intracellular $\mathrm{Ca}^{2+}$ accumulation (Arun et al., 2013; Abdul-Muneer et al., 2014). Although bTBI is considered a diffuse injury, a majority of damage from our model is localized to the prefrontal cortex (PFC) (Turner et al., 2013b), where the brain impacts the skull on the contra coup side of exposure (Zhu et al., 2010; Zhu et al., 2013). $\mathrm{Ca}^{2+}$ perturbations are known to cause endoplasmic reticulum (ER) stress and trigger the unfolded protein response (UPR) (Zhang and Kaufman, 2008; Walter and Ron, 2011). Although the UPR has been reported in a model of controlled cortical impact TBI (Farook et al., 2013), the mechanisms of cellular fate are not yet fully elucidated.

Neuropsychiatric behaviors measured in animal models, such as impulsive-like behaviors, are a strong indicator of damage to the rodent PFC (Bidzan et al., 2012; Johnson et al., 2013a). Similar personality disorders are often observed in human bTBI patients as well, 
providing an important research parallel (Vaishnavi et al., 2009). We propose that our clinicallyrelevant blast model allows us to investigate the process of ER stress and how this response relates to apoptosis and neuropsychiatric disorders.

A common downstream component of the UPR is the C/EBP homologous protein (CHOP), which becomes upregulated during sustained cellular stress to maintain ER homeostasis (Walter and Ron, 2011). The levels of CHOP dictate whether a cell can effectively repair itself, or proceed to apoptosis by regulating pro- and anti-apoptotic mechanisms (McCullough et al., 2001; Galehdar et al., 2010). Acute phase activation of the protein kinase R-like ER kinase (PERK) UPR pathway, and its' downstream component growth arrest and DNA damageinducible protein 34 (GADD34), helps to maintain CHOP within an ideal range to promote cellular repair (Salminen and Kaarniranta, 2010).

Under sustained ER stress, intracellular $\mathrm{Ca}^{2+}$ accumulation can trigger apoptosis through a separate cascade involving calpain-mediated Caspase-12 cleavage (Nakagawa et al., 2000). This mechanism is considered separate from the UPR (Badiola et al., 2011), even though both apoptotic cascades share Caspase-3 cleavage as a final common step in undergoing apoptosis (Szegezdi et al., 2006). Using our model, we are interested in determining the mechanism by which bTBI triggers apoptosis and how this relates to the pathology of chronic disease. This study investigates acute BBB disruption, ER stress mechanisms, apoptosis and impulsivelike behavior following a single blast injury. It has been proposed that bTBI pathophysiology is partly mediated by alterations in BBB permeability (Chen et al., 2013c), which may induce ER stress and trigger the UPR (Begum et al., 2014). The ER stress modulator, salubrinal (SAL), has been used to investigate downstream components of the PERK pathway (Sokka et al., 2007). Our hypothesis is that SAL manipulation of the PERK pathway would maintain CHOP expression 
within a protective threshold. Balancing CHOP expression should regulate apoptosis and mitigate impulsive-like behavior indicative of blast injury (Kamnaksh et al., 2011). Therefore, treatment options should consider the UPR mechanism for the detrimental sequelae of neuropsychiatric disorders.

\section{METHODS}

\section{Animals}

All procedures involving animals $(\mathrm{N}=144)$ were approved by the Institutional Animal Care and Use Committee of West Virginia University and were performed according to the principles of the Guide for the Care and Use of Laboratory Animals. This work used young adult male Sprague-dawley rats acquired from Hilltop Lab Animals (Hilltop Lab Animals, Inc.) and weighed $\sim 300-350 \mathrm{~g}$ at the time of blast and sacrifice. Animals were acclimated for 1 week prior to experimental use and were housed under $12 \mathrm{~h}$ light/dark conditions with food and water available ad libitum.

\section{Blast Overpressure Exposure}

Prior to blast exposure, animals were anesthetized with $4 \%$ isoflurane (Halocarbon). The blast was delivered to the right side of the head with the animal's body oriented perpendicular to the blast tube, and with the peripheral organs protected by a polyvinyl chloride pipe shield. The animals were exposed to a mild blast ( $0.005 "$ membrane; $\sim 15$ psi on incident recordings; $\sim 50$ psi on reflected recordings), which was determined, in previous work, to produce microscopic neuronal injury to the contra coup (left) side of the brain, with no signs of hemorrhagic injury under gross examination (Turner et al., 2013b). Immediately following blast exposure, animals

were returned to a holding cage equipped with a homeothermic heating blanket equipped with a 
rectal thermometer to maintain body temperature at $37^{\circ} \mathrm{C}$. Once basic reflexes were restored, animals were returned to the home cage.

\section{Salubrinal Administration}

A stock solution of SAL (Tocris) was made in $0.5 \%$ dimethyl sulfoxide (DMSO). Such a low concentration of DMSO was chosen to avoid neurological effects (Methippara et al., 2012). SAL was diluted to $100 \mathrm{uM}$ to effectively manipulate the UPR (Boyce et al., 2005). A DMSO concentration of $0.5 \%$ was administered to control and bTBI rats 30 min prior to anesthesia. SAL was aliquoted for each intraperitoneal injection at a dose of $1 \mathrm{mg} / \mathrm{kg}$ (Sokka et al., 2007; Liu et al., 2014b). SAL was administered 30 min prior to anesthesia (SAL), or anesthesia followed by blast exposure (SAL+bTBI).

\section{Blood Brain Barrier Permeability Assessment}

Animals in the BBB assessment group $(\mathrm{N}=16)$ were evaluated at three time points following blast exposure: $0.5,6,24 \mathrm{~h}$, and control $(n=4)$. Following blast exposure, BBB permeability was assessed using Evan's Blue (EB; Sigma). EB binds to albumin and is a marker used to detect BBB permeability (Yen et al., 2013). We had previously shown changes in BBB permeability and tight junction protein expression with our model (Lucke-Wold et al., 2014c). Animals were anesthetized with $4 \%$ isoflurane and maintained with $2 \%$ isoflurane throughout the procedure. Saline containing EB $(2 \%, 5 \mathrm{ml} / \mathrm{kg})$ was administered intravenously (femoral vein) 30 min before perfusion. The rats were then transcardially perfused with $0.9 \%$ saline for 15 min and brains excised for raw imaging. The PFC was then dissected out and separated by hemisphere. The samples were weighed and homogenized in $0.5 \mathrm{ml}$ of $50 \%$ trichloroacetic acid (Sigma). The samples were then incubated for $24 \mathrm{~h}$ at $37^{\circ} \mathrm{C}$ before being centrifuged at $10,000 \mathrm{x} \mathrm{g}$ for $10 \mathrm{~min}$ at $4^{\circ} \mathrm{C}$. The supernatant was measured by absorbance spectroscopy at $620 \mathrm{~nm}$. Calculations were 
based on an external standard reading and extravasated dye was expressed as ng EB / mg brain tissue.

\section{Quantitative Real-time Polymerase Chain Reaction}

Rats $(\mathrm{N}=48)$ for the gene analysis group were randomly divided into one of two groups: time course $(n=24)$ and control $(n=24)$. The time course group consisted of six time-points

post-bTBI: $1.5,3,6,12,24$, and $72 \mathrm{~h}(n=4)$. The control group (anesthetized only) used rats for all six time point from the time course study $(n=4)$. All animals were anesthetized with $4 \%$ isoflurane and euthanized via decapitation in protease/phosphatase cocktail (Plattner et al., 2006). Brains were rapidly removed with the PFC dissected out, separated by hemisphere and flash frozen in liquid nitrogen. Total RNA was isolated in TRI-Reagent (Sigma) and was tested for quantity and quality using a Nanodrop 2000c spectrophotometer (Thermo Scientific). Reverse transcription was conducted using a high capacity reverse transcription kit (Applied Biosystems). Real-time PCR analyses were performed using a 7500 Real-Time PCR system (Applied Biosystems) in combination with TaqMan® chemistry using the following oligonucleotide primer sets: activating transcription factor 4 (atf4) (Rn00824644_g1), CHOP (ddit3) (Rn00492098_g1), GADD34 (ppp1R15A) (Rn00591894_m1), Glial fibrillary acidic protein (gfap) (Rn00566603_m1) with 18s rRNA (Hs99999901_s1) used as an endogenous control (Applied Biosystems). Changes in gene expression were determined using the $(\Delta \Delta \mathrm{Ct})$ method with a threshold cycle value of 0.2 normalized to $18 \mathrm{~s}$ rRNA.

\section{Western Blotting}

Rats $(\mathrm{N}=20)$ for the protein analysis group were randomly divided into four different experimental groups: (1) control, (2) SAL, (3) bTBI $24 \mathrm{~h}$, and (4) SAL+bTBI $24 \mathrm{~h}(n=5)$. Animals were euthanized and had tissue prepared as previously described. Protein samples were 
prepared by sonication in hot $\left(85-95^{\circ} \mathrm{C}\right) 1 \%$ sodium docecyl sulfate (SDS; Sigma) as previously described (O'Callaghan and Sriram, 2004). The protein concentration of each sample was measured using a bicinchoninic acid (BCA) protein assay kit (Pierce). Samples were run using $30-50 \mu \mathrm{g}$ of protein/well, depending on the primary antibody, using pre-cast Bolt ${ }^{\circledR}$ Bis-Tris Plus gels (Life Technologies) in combination with 2X Lammeli sample buffer. Gels were run using the Bolt@ Mini tank system (Life Technologies) and transferred to polyvinylidene fluoride membranes (Bio-Rad) using wet electrophoretic transfer cells (Bio-Rad). Membranes were incubated with the following primary antibodies all raised in rabbit: CHOP 1:1000 (Cell Signaling), Caspase-12 1:1000 (Cell Signaling), Caspase-3 1:750 (Cell Signaling), and GADD34 1:1000 (Pierce) overnight at $4^{\circ} \mathrm{C}$. Anti-rabbit IgG horseradish peroxidase (HRP)-linked antibody (Cell Signaling) was used as a secondary antibody at a concentration of 1:2000 with gentle shaking for $2 \mathrm{~h}$ at room temperature. The rabbit monoclonal antibody $\beta$-actin (Cell Signaling) was used as an endogenous control for all samples at a concentration of 1:10,000. Molecular weight determination was conducted using the SeeBlue ${ }^{\circledR}$ Plus2 Pre-stained Standard (Life Technologies). Imaging was conducted using LumiGLO chemiluminescent substrate (Cell Signaling) according to manufacturer's instructions. Images were converted to 8-bit and analyzed using densitometry with background subtraction and normalized to $\beta$-actin using ImageJ software (NIH).

\section{Immunohistochemistry Preparation}

Rats $(\mathrm{N}=12)$ used for the immunohistochemistry (IHC) group were randomly divided into three experimental groups: (1) control, (2) bTBI $24 \mathrm{~h}$, and (3) SAL+bTBI $24 \mathrm{~h}(n=4)$. Histological samples were prepared as previously described (Lucke-Wold et al., 2014c). Briefly, the animals were anesthetized by inhalation of $4 \%$ isoflurane and maintained with $2 \%$ isoflurane 
throughout the procedure. Animals were then transcardially perfused with $0.9 \%$ ice-cold saline for $5 \mathrm{~min}$ followed by $10 \%$ formalin for $15 \mathrm{~min}$. The brains were subsequently removed and placed in $10 \%$ formalin solution for $24 \mathrm{~h}$. Following fixation, the PFC was sectioned on a brain block at $4 \mathrm{~mm}$ increments. Sections were then processed with a Tissue Tek VIP 5 automatic processor (Sakura Finetek), and embedded with Tissue Tek TEC (Sakura Finetek) as previously described (Turner et al., 2012b). Slices $(10 \mu \mathrm{m})$ were prepared with the Leica RM2235 microtome (Leica Microsystems), mounted onto slides, and heat fixed for fluorescent staining. A total of 46 coronal sections were prepared per animal.

\section{Immunohistochemistry Staining}

Paraffin was dissolved from slides with 5 min washes in xylene, 100\% EtOH, and 95\% EtOH followed by 5 min rehydration in $\mathrm{dH}_{2} \mathrm{O}$. The slides were then quenched with $10 \%$ methanol and $10 \% \mathrm{H}_{2} \mathrm{O}_{2}$ in Dulbecco's phosphate buffered saline (DPBS) for $15 \mathrm{~min}$. After quenching, slides were rinsed three times in DPBS for 5 min each. The slides were then placed in permeabilizing solution (1.8\% L-lysine, $4 \%$ horse serum, and $0.2 \%$ Triton X-100 in DPBS) for $30 \mathrm{~min}$. Slides were allowed to dry and the brain slices were circumscribed. Tissue was incubated with primary antibody in DPBS with $4 \%$ horse serum overnight. Tissues were washed three times in DPBS and incubated in secondary fluorescent antibody for $3 \mathrm{~h}$. Tissues were then rinsed three times in DPBS and dried overnight. Vectashield mounting media was used to fix the coverslip (Vector Labs). When staining for co-localization, a second set of primary and secondary antibodies were used prior to fixing the coverslip. Primary antibodies were GFAP rabbit mAB (Dako) 1:500, CHOP mouse mAB (Cell Signaling) 1:1600, Caspase-3 rabbit polyAB (Abcam) 1:1000, Caspase-12 mouse polyAB (Cell Signaling) 1:1000, and microtubule associated protein 2 (MAP2) rabbit mAB (Millipore) 1:1000. Secondary antibodies were diluted 
1:100 in DPBS and included Alexa Fluor 488 goat anti-rabbit (Life Technologies), Alexa Fluor 594 goat anti-rabbit (Life Technologies), Alexa Fluor 488 goat anti-mouse (Life Technologies), and Alexa 594 goat anti-mouse (Life Technologies). Imaging was performed with a Zeiss Axio Imager 2 (Carl Zeiss Microscopy).

Immunohistochemistry Quantification

Corrected total cell fluorescence (CTCF) was calculated using ImageJ software (NIH).

Briefly, 12 randomly selected areas of the left PFC were outlined and measured, with fluorescent density compared to background readings. Slides from each region were randomly selected by an observer blinded to the experimental groups. The density was adjusted per mean area to give corrected total cell fluorescence (Lucke-Wold et al., 2014c). For co-localization quantification, the ImageJ plugin titled Just Another Co-localization plugin was utilized to calculate a Pearson's coefficient as well as an overlap coefficient for each sample (Beerten et al., 2012).

\section{Terminal Deoxynucleotidyl Transferase-mediated dUTP Nick End Labeling}

Slides for staining were prepared, sectioned and mounted as previously described. We used an apoptosis detection kit to assess the left PFC at $24 \mathrm{~h}$ following blast exposure. Staining for apoptosis was completed using the TACS 2 TdT-Dab in situ Apoptosis Detection kit (Trevigen) according to manufacturer's instructions. In brief, paraffin embedded slices were deparaffinized and rehydrated with five minute incubations in xylenes, 100\% EtOH, 95\% EtOH, and deionized water each. The slices were then immersed in phosphate buffered saline for 10 minutes followed by treatments with Proteinase K, quenching solution, labeling buffer, and labeling reaction mixture. The sample was then covered in Strep-HRP solution for ten minutes, washed, and then immersed in 3,3'-Diaminobenzidine (DAB) solution for seven minutes. The slices were counterstained with $1 \%$ methyl green and dehydrated with 10 dips in deionized 
water, $95 \% \mathrm{EtOH}, 100 \% \mathrm{EtOH}$, and xylenes. The slides were coverslipped using Permount ${ }^{\mathrm{TM}}$ (Sigma) mounting media and glass coverslips. For quantification of DAB staining, regions of the PFC were randomly selected for rats from the different treatment groups. An observer blinded to experimental group, randomly selected 100 total cells. The number of positive cells was reported as a fraction of total cells counted.

\section{Elevated Plus Maze}

Impulsive-like behavior can be measured with increased exploratory behavior in a rodent model of anxiety (Mosienko et al., 2012). Four groups of rats $(\mathrm{N}=48)$ were subject to behavioral analysis: control, SAL, bTBI $7 \mathrm{~d}$, and SAL+bTBI $7 \mathrm{~d}(n=12)$. The elevated plus maze (EPM) was set at a height of $60 \mathrm{~cm}$ from the floor. The two open arms intersected perpendicular to the two closed arms. Each arm was $50 \mathrm{~cm}$ x $10 \mathrm{~cm}$. The closed arms were encased by black siding $30 \mathrm{~cm}$ tall. Each rat was placed in the middle of the elevated plus maze facing an open arm and tracking was performed for 5 min with AnyMaze ${ }^{\mathrm{TM}}$ software (Version 4.7, Stoelting), which pinpointed the location of the animal's head and body continuously throughout the testing trial. The percent time spent in the open arms, speed, closed arm entries and movement were all recorded and quantified. Increased percent time spent in the open arms was considered a sign of impulsive-like behavior, as previously described (Mosienko et al., 2012; Johnson et al., 2013a).

\section{Data Analysis}

Data were analyzed using GraphPad Prism 5.0 (GraphPad Software, Inc.). All data points are shown as mean \pm s.e.m. Statistical differences between control and experimental groups were determined by using ANOVA with a Dunnett's, Tukey's or Bonferroni's post-hoc tests. A twotailed Student's $t$-test was used when comparing two conditions only. For DAB staining, a chisquare analysis was used to compare between groups. A power analysis was conducted for all 
experiments with an $\alpha$ of 0.05 and a $\beta$ of 0.2 (DSS Research Power Analysis). Sample sizes were determined by the sample effect with behavioral data being set at 0.4 and all other data being set at 0.3 . A value of $p<0.05$ was considered statistically significant for all data analyzed.

\section{RESULTS}

The physical force of bTBI can shear axons (Raghupathi and Margulies, 2002), and rupture micro-vessels (Arun et al., 2013). These primary effects can cause neurons to rapidly depolarize and activate voltage gated $\mathrm{Ca}^{2+}$ channels, thereby increasing intracellular $\mathrm{Ca}^{2+}$ (Gurkoff et al., 2013). Studies using other models of neurotrauma have shown marked BBB dysfunction (Abdul-Muneer et al., 2014), ER stress activation (Farook et al., 2013), and apoptosis (Sabirzhanov et al., 2014). SAL is known to prevent the dephosphorylation of eukaryotic initiation factor 2 alpha (eIF2 $\alpha$ ) (Boyce et al., 2005); however, this agent may directly affect other mechanisms of ER stress (Zhang et al., 2014a) or apoptosis (Kessel, 2006). Figure 4.1 portrays how bTBI may trigger the UPR, as well as the proposed effects of SAL.

\section{Contra Coup Vascular Disruption}

The suggestion has been made previously that BBB disruption, or loss of micro-vessel endothelium integrity, may be an inciting event for the molecular changes frequently induced following neurotrauma (Abdul-Muneer et al., 2013; Arun et al., 2013). This may be particularly relevant in models of blast injury in which a pressure wave, and associated surge in vascular flow to the brain, may induce microvascular changes manifested as BBB disruption (Sosa et al., 2013). Following BBB disruption, EB binds to albumin and diffuses into the brain in a locationspecific manner consistent with disruption (Yang et al., 2013). Recently, we have shown changes

in BBB permeability and tight junction protein expression using our blast model (Lucke-Wold et 
al., 2014c). To further address these issues, we used EB extravasation to assess the brain vasculature of the PFC following blast exposure.

One-way ANOVA revealed a significant difference in EB absorbance in the left PFC following blast exposure $\left(\mathrm{F}_{(3,12)}=31.350, p<0.001\right)$. Dunnett's post-hoc analyses revealed bTBI significantly increased EB absorbance in the left PFC at $0.5 \mathrm{~h}(q=8.844, p<0.001)$ and 6 $\mathrm{h}(q=3.491, p<0.05)$ (Figure 4.2A). Raw images of extracted brains at varying time points $(0.5,6$, and $24 \mathrm{~h})$ demonstrate BBB disruption, particularly prominent in the left PFC (Figure 4.2B). The images provide vivid proof of a contra coup style of injury following bTBI. Because our model's blast exposure produced no signs of hemorrhagic transformation under gross examination at this severity (Turner et al., 2013b), we are certain that these findings indicate a loss in BBB integrity on a microscopic scale.

\section{Blast upregulates stress response markers}

To investigate gene changes following blast exposure, we performed quantitative realtime PCR. A time course was employed to measure acute and sub-acute changes in mRNA abundance of four stress response genes (atf4, ddit3, ppplR15A and gfap). Atf4 encodes for ATF4, ddit3 encodes for CHOP, ppp1R15A encodes for GADD34, and gfap encodes for GFAP. Two-Way ANOVA revealed significant differences in left PFC atf4 mRNA abundance between treatment, time, and interaction $(p<0.05)$. Blast exposure significantly increased the mRNA abundance of atf4 in the left PFC at $3 \mathrm{~h}(t=7.694, p<0.001)$ (Bonferroni's post-hoc analysis)

(Figure 4.3A). Two-Way ANOVA also revealed significant differences in left PFC ddit3 mRNA abundance between treatment, time, and interaction $(p<0.05)$. Bonferroni's post-hoc analysis revealed a significant increase in left PFC $d$ dit 3 mRNA abundance at $3 \mathrm{~h}$ post-bTBI $(t=7.989, p$ $<0.001$ ) (Figure 4.3B). To further validate our blast model induces a contra coup form of injury 
we also measured mRNA abundance of atf4 and ddit3 in the right PFC. Indeed no differences were observed in the right PFC for atf4 (Figure 4.3C), or ddit3 (Figure 4.3D). Left PFC mRNA abundance for both atf4 and ddit3 quickly returned to baseline by $6 \mathrm{~h}$ and remained at this level through 72 h, implying an acute phase stress response (Figure 4.3A-B).

Two-Way ANOVA revealed significant differences in the left PFC ppp1R15A mRNA abundance between time and interaction $(p<0.05)$. Blast exposure significantly increased the mRNA abundance of ppp1R15A in the left PFC at $6 \mathrm{~h}(t=6.022, p<0.001)$ (Bonferroni's posthoc analysis) (Figure 4.3E). Two-Way ANOVA also revealed significant differences in left PFC gfap mRNA abundance between treatment and time $(p<0.05)$. Bonferroni's post-hoc analysis revealed a significant increase in left PFC gfap mRNA abundance at 24 h post-bTBI $(t=4.081, p$ $<0.01$ ) (Figure 4.3F). Results indicate blast exposure imparts downstream upregulation of UPR marker, GADD34, with trailing astrocyte activation at a later time point. Astrocyte activity is indicative of neuroinflammation and early stages of cell death.

\section{Salubrinal attenuates ER stress markers}

SAL is a research tool known to inhibit the UPR in vitro (Boyce et al., 2005) and in vivo (Sokka et al., 2007). In particular, SAL prevents the dephosphorylation of eIF2 $\alpha$ by the GADD34 phosphatase complex formation (Hetz et al., 2013). We used this tool prior to blast exposure to alter ER stress and to examine any effects modulating the UPR. Western blot was used to compare $\mathrm{CHOP}$ expression in the left and right PFC to further support contra coup injury indicative of bTBI (Zhu et al., 2010; Zhu et al., 2013). A significant increase in CHOP expression was measured in the left PFC at $24 \mathrm{~h}$ post-bTBI $(t=2.625, p<0.05)$ (Figure 4.4A), but not in the right PFC $(t=1.487, p>0.05)$ (Figure 4.4B) (Two-Tailed Student's $t$ Test). A significant difference in $\mathrm{CHOP}$ expression was observed with a One-Way $\operatorname{ANOVA}\left(\mathrm{F}_{(3,12)}=\right.$ 
5.775, $p<0.01)$. At $24 \mathrm{~h}$, a significant increase in CHOP expression was seen in the left PFC of bTBI rats $(t=3.705, p<0.05)$, but was attenuated in the left PFC of SAL+bTBI rats $(t=3.105, p$ $<0.05$ ) (Bonferroni's post-hoc analysis) (Figure 4.4C). These findings indicate SAL given alone does not alter the constitutively active form of CHOP; however, stress-activated $\mathrm{CHOP}$ can be attenuated when SAL is administered prior to blast exposure.

ER stress markers Caspase-12 and GADD34 were investigated to determine if SAL had any effects on cellular fate following blast exposure. One-Way ANOVA revealed a significant difference in the proteolytic processing of Caspase- $12\left(\mathrm{~F}_{(3,16)}=10.230, p<0.001\right)$ at $24 \mathrm{~h}$ postbTBI. Caspase-12 cleavage significantly increased in the left PFC of bTBI rats $(t=3.696, p<$ 0.05), as well as in the left PFC of SAL+bTBI rats $(t=3.393, p<0.05)$ (Bonferroni's post-hoc analysis) (Figure 4.4D). A One-Way ANOVA revealed a significant difference in GADD34 protein expression $\left(\mathrm{F}_{(3,16)}=5.216, p<0.05\right)$ following blast exposure. GADD34 protein expression significantly increased in the left PFC of bTBI rats at $24 \mathrm{~h}(t=3.136, p<0.05)$. This effect was mitigated when SAL was administered prior to blast exposure $(t=3.107, p<0.05)$ (Bonferroni's post-hoc analysis) (Figure 4.4E). Findings suggest SAL may control cellular fate through the modulation of ER stress in a bTBI model.

\section{Salubrinal modulates ER-mediated apoptosis}

CHOP, Caspase-12 and Caspase-3 are all markers of ER-mediated apoptosis (Nakagawa and Yuan, 2000; Nakagawa et al., 2000; Tabas and Ron, 2011). Caspase-12 is initially cleaved by ER stress through a perturbation in $\mathrm{Ca}^{2+}$ homeostasis; thereby, activating Caspase- 3 and triggering apoptosis (Bernales et al., 2012). To further confirm that bTBI increases CHOP expression, we conducted IHC fluorescent staining in the left PFC and measured corrected total 
cell fluorescence (CTCF). We also measured CTCF for Caspase-12 and Caspase-3 in the left PFC.

Consistent with Western blot findings, IHC analyses showed a significant difference in CTCF for CHOP after blast exposure (One-Way ANOVA; $\mathrm{F}_{(2,12)}=34.390, p<0.001$ ).

Bonferroni's post-hoc analyses revealed bTBI significantly increased CHOP CTCF ( $t=8.099, p$ $<0.001)$, while SAL pre-administration reduced CHOP CTCF $(t=5.592, p<0.001)$ in the left $\mathrm{PFC}$ at $24 \mathrm{~h}$ (Figure 4.5A). IHC analyses also showed that bTBI portrays a significant difference in CTCF for Caspase-12 (One-Way ANOVA; $\mathrm{F}_{(2,12)}=22.03, p<0.001$ ), and Caspase-3 (OneWay ANOVA; $\left.\mathrm{F}_{(2,12)}=12.490, p<0.01\right)$. Bonferroni's post-hoc analyses revealed a significant increase in CTCF for Caspase-12 at $24 \mathrm{~h}$ in the left PFC of bTBI rats $(t=6.066, p<0.001)$, as well as SAL+bTBI rats $(t=5.366, p<0.001)$ (Figure 4.5B). SAL also attenuated $(t=3.967, p<$ $0.01)$ the increased Caspase-3 CTCF $(t=4.615, p<0.01)$ at $24 \mathrm{~h}$ post-bTBI in the left PFC (Figure 4.5C). These findings suggest SAL plays an important role in the control of cellular fate through CHOP- and Caspase-3-dependent mechanisms following blast exposure.

We employed IHC colocalization to determine cell-specific UPR activation. CHOP displayed a moderate colocalization with the neuron-specific MAP2 in the left PFC at $24 \mathrm{~h}$ postbTBI (Pearson's coefficient, $r=0.536$ ) (Figure 4.6). CHOP displayed a weak colocalization with MAP2 in the control (Pearson's coefficient, $r=0.371$ ) and SAL+bTBI groups (Pearson's coefficient, $r=0.233)($ Figure 4.6). The results suggest blast exposure increases $\mathrm{CHOP}$ protein expression in neurons of the left PFC at $24 \mathrm{~h}$, and also supports the protein and gene data shown previously for $\mathrm{CHOP}$.

IHC colocalization was also used to determine if $\mathrm{CHOP}$ and Caspase-12 activation occur in the same cells. We observed a very weak colocalization between CHOP and Caspase-12 in the 
left PFC of control rats (Pearson's coefficient, $r=0.177$ ). We discovered a moderate colocalization between CHOP and Caspase-12 at $24 \mathrm{~h}$ in the left PFC of bTBI rats (Pearson's coefficient, $r=0.537$ ) (Figure 4.7), as well as a moderate colocalization in the left PFC of SAL $+b T B I$ rats (Pearson's coefficient, $r=0.677$ ) (Figure 4.7). These results suggest $\mathrm{CHOP}$ and Caspase-12 to be active within the same cell after blast exposure. Colocalization strengths also suggest SAL may not have a direct effect on Caspase-12 cleavage.

\section{Salubrinal mitigates Caspase-3 cleavage}

We wanted to determine if SAL had an effect on Caspase-3 cleavage. Caspase-3 cleavage is one of the final steps of the apoptotic cascade and is a common indicator used to assess cell death after TBI (Clark et al., 2000). One-Way ANOVA revealed a significant difference in Caspase- 3 cleavage at $24 \mathrm{~h}$ post-bTBI $\left(\mathrm{F}_{(3,16)}=5.533, p<0.01\right)$. Caspase- 3 cleavage significantly increased in the left PFC of bTBI rats $(t=3.251, p<0.05)$, but was not significantly increased in the left PFC of SAL+bTBI rats $(t=1.200, p>0.05)$ (Bonferroni's post-hoc analysis) (Figure 4.8A). These results suggest blast exposure elicits cell death through apoptosis. The results also suggest that ER stress plays an important role in the control of cellular fate following blast injury.

We wanted to determine if our blast model produced cell death, and if SAL preadministration would reduce bTBI-induced cell death. To do this we employed a terminal deoxynucleotidyl transferase-mediated dUTP nick end labeling assay that utilizes DAB to stain cells undergoing the initial stages of apoptosis. We noticed cell membrane blebbing following blast exposure in the left PFC, which indicates early signs of apoptosis (Clark et al., 1997). DAB staining revealed increased apoptosis in bTBI rats compared to control rats (Figure 4.8B,C). Interestingly, SAL+bTBI rats displayed less DAB positive staining compared to bTBI rats 
(Figure 4.8D). The ratio of positive stained cells for control $=3 / 100$, bTBI $=26 / 100$, and

$\mathrm{SAL}+\mathrm{bTBI}=3 / 100 \cdot \chi^{2}=33.456$ with 2 degrees of freedom and $p<0.001$.

\section{Impulsive-like behavior ameliorated by salubrinal}

We assessed the percent time spent in the open arms of the EPM to measure changes in impulsive-like behavior (Mosienko et al., 2012). Track plots from the Anymaze ${ }^{\mathrm{TM}}$ analysis were included for each experimental group to visually support the behavioral changes (Figure 4.9A). A One-Way ANOVA exhibited a significant difference in the percent time spent in the open arms at $7 \mathrm{~d}$ post-bTBI $\left(\mathrm{F}_{(3,44)}=4.250, p<0.05\right)$. Tukey's post-hoc analysis revealed a significant increase in the percent time spent in the open arms between bTBI rats and SAL alone rats $(q=3.816, p<0.05)$. SAL given prior to bTBI significantly reduced the percentage of time spent in the open arms compared to the $7 \mathrm{~d}$ post-bTBI group $(q=4.657, p<0.05)$ (Tukey's posthoc analysis) (Figure 4.9B). No significant differences were observed between groups in speed $(p>0.05)$ (Figure 4.9C), closed arm entries $(p>0.05)$ (Figure 4.9D), or total movement $(p>$ 0.05) (Figure 4.9E) (Tukey’s post-hoc analysis). Overall, these results indicate blast exposure may increase impulsive-like behavior, and that ER stress modulation may play a role in the manipulation of this type of behavior.

\section{DISCUSSION}

Data from the current study provides evidence that blast exposure disrupts the BBB and increases PERK-mediated ER stress in the left PFC. Impulsive-like behavior, a neuropsychiatric symptom associated with PFC damage (Johnson et al., 2013a), was demonstrated by rats exposed to bTBI through their increased exploration in the open arms of the EPM. IHC results confirm bTBI increases the expression of neuronal apoptosis. ER stress modulation, influences cellular 
fate and ameliorates impulsive-like behavior indicative of blast exposure. Overall, these results suggest a possible mechanistic link between ER stress, apoptosis and neuropsychiatric disease. Previous groups have shown that disruptions in the BBB by various insults such as ischemic stroke and epilepsy can cause increased micro-vessel permeability (Kaya and Ahishali, 2011).

The external forces of TBI can cause rapid depolarization of neuronal cell membranes; thereby, activating voltage gated $\mathrm{Ca}^{2+}$ channels and increasing intracellular $\mathrm{Ca}^{2+}$ levels (Gurkoff et al., 2013; Begum et al., 2014). Until recently, it was unknown to what extent blast injury disrupts the BBB. A mild exposure ( 15 psi on incident recordings) from our clinically-relevant blast model previously exhibited no signs of brain hemorrhage under gross examination (Turner et al., 2013b). A new study showed BBB disruption at $6 \mathrm{~h}$ following a mild intensity blast ( 17.8 psi) in Sprague-Dawley rats (Abdul-Muneer et al., 2013). Our results indicate a more acute disruption in BBB permeability shown at $0.5 \mathrm{~h}$, which is an earlier documentation of $\mathrm{BBB}$ disruption following bTBI. The rapid increase in permeability from blast injury is thought to result from an intracranial pressure spike (Chen et al., 2013c), and may be an important primary effect driving cellular stress (Arun et al., 2013).

Increased intracellular $\mathrm{Ca}^{2+}$ triggered ER stress and activated the UPR in other models of neuronal injury (Osada et al., 2009). Similarly, mild neurotrauma was shown to activate other adaptive arms of the UPR in mice (Rubovitch et al., 2011a). The UPR is unique in that depending on the time and duration of the response, different arms of the pathway are activated (Rubovitch et al., 2011a). If the UPR lasts too long, a switch from neuroprotection to apoptosis occurs (Nakagawa et al., 2000; Urra et al., 2013). Apoptosis is not purely detrimental to the damaged brain considering the heightened energy demands following neurotrauma. By limiting energy expended on severely damaged cells, the brain can preserve function to surviving cells. 
Blast-induced CHOP elevation, along with increased Caspase-12 and Caspase-3 cleavage, suggests a neuronal shift from the repair response to apoptosis. Modulation of the ER stress response with SAL has been shown to attenuate CHOP expression (Zhang et al., 2014a) and limit apoptosis in other models of neuronal injury (Sokka et al., 2007; Nakka et al., 2010). SAL's effects on Caspase-12 remain controversial where some studies claim SAL mitigates Caspase-12 cleavage (Liu et al., 2012), while other studies claim SAL promotes cleavage (Gao et al., 2013). Furthermore, it is important to note that Caspase-12 is regulated through a calpaindependent process (Nakagawa and Yuan, 2000) independent from the PERK-mediated UPR (Badiola et al., 2011). In our bTBI model, we show that SAL mitigates CHOP expression and reduces Caspase-3-mediated cell death with no effect on Caspase-12 cleavage. This suggests that SAL may not have a direct effect on Caspase-12 cleavage, but still plays a role in the control of cellular fate. Our findings, along with the findings of others, provide evidence for new ways to examine cellular stress and apoptosis in models of neuronal injury.

Neurotrauma is also intimately associated with post-injury changes in behavior (Schroeter et al., 2007). Blast exposure increases impulsive-like behavior in adult male Sprague Dawley rats as shown in the EPM data. This finding may correlate with acute behavioral findings seen in soldiers following head injury (Menon et al., 2010). Interestingly, SAL attenuated the percentage of time spent in the open arms of the EPM following bTBI. These findings suggest that SAL may have a modulatory effect on impulsive-like behavior indicative of PFC damage through modulation of ER stress. While significant advances have been made in understanding the acute pathophysiology of blast exposure, it remains unclear how bTBI leads to the development of neuropsychiatric disorders (Tweedie et al., 2013). 
Emerging evidence indicates that the UPR may be one potential mechanism linking acute neuronal injury and chronic disease pathology (Scheper and Hoozemans, 2013). In various models of neurodegenerative disease, evidence of PERK-mediated ER stress activation has been implicated (Costa et al., 2012; Ho et al., 2012; Nijholt et al., 2012). Moreover, experimental work using other models of neurotrauma have shown elevated UPR markers (Farook et al., 2013; Begum et al., 2014) and behavioral deficits (Goldstein et al., 2012; Petraglia et al., 2014c). Based on our findings, the elevation of UPR markers concurrently with Caspase-3 cleavage suggests neuronal apoptosis, which has been implicated as an early indicator of chronic disease pathology (McKee et al., 2009). As such, our study provides a correlative link between blast-induced UPR activation and neuropsychiatric disorder development. Future studies, likely using genetically altered animals, or additional pharmacologic inhibitors, are required to examine the precise role of ER stress in the development of chronic disease following neurotrauma. Similarly, investigating PERK-mediated ER stress in pathologic clinical specimens will further clarify the potential role of the pathway in neuropsychiatric disease development.

Long-term studies utilizing both a single and repetitive injury model are warranted in order to examine not only chronic disease processes associated with ER stress but also the effect of repetitive injury on the activation of the ER stress response. Finally, the contribution of the other two adaptive arms of the UPR following blast injury remains to be elucidated. All three adaptive arms of the UPR share CHOP as a signal mediator and is of particular interest due to the overlapping nature of the pathways and the potential for modulation of signaling in a biphasic manner. We are also interested in the role of axonal shearing as a result of blast exposure. We have shown previously that a majority of damage from our blast model occurs in the corpus 
callosum (Turner et al., 2013b). This is considered another primary effect of blast exposure and warrants future investigation using our model.

Blast exposure disrupts the BBB and triggers ER stress in the left PFC. Rats exposed to blast exhibit more impulsive-like behavior and display markers of neuronal apoptosis. When bTBI rats were given the ER stress modulator, SAL, markers of apoptosis and impulsive-like behavior were both attenuated. The cells that survive the initial primary injury of bTBI are those that we seek to protect from secondary injury mechanisms. Future studies linking ER stress to chronic disease are ongoing and could provide new molecular targets for treatment following blast injury. 


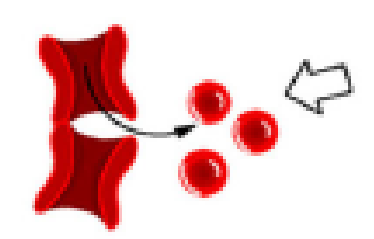

\section{Intracranial Pressure Spike}

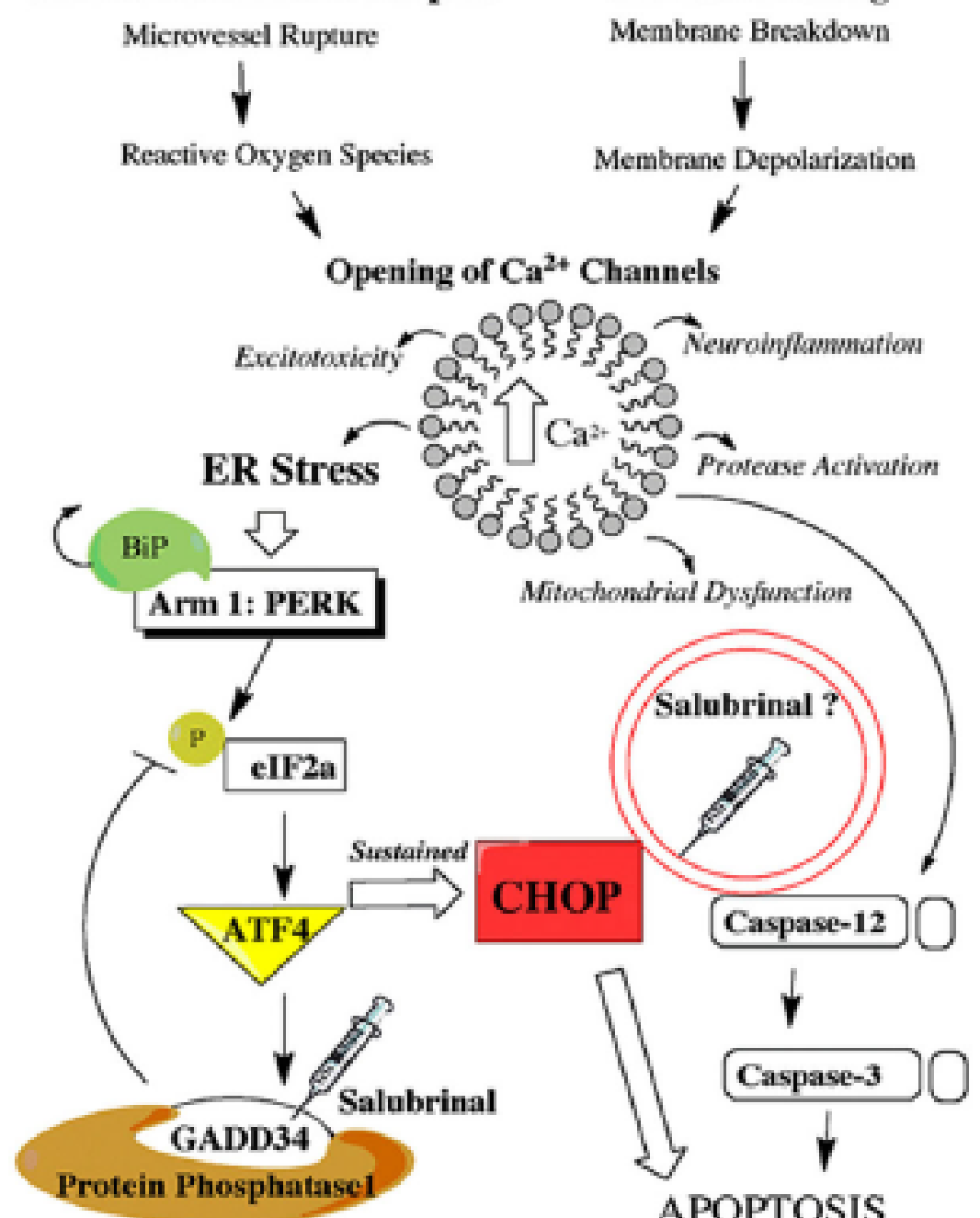

Figure 4.1. Schematic shows primary and secondary effects of blast injury. Blast-induced traumatic brain injury (bTBI) can burst brain microvessels and shear axons. Both primary effects lead to increased intracellular calcium levels which triggers a variety of secondary effects including endoplasmic reticulum (ER) stress. ER stress activates the unfolded protein response (UPR) which consists of three separate adaptive arms that play a time-dependent role in maintaining cellular homeostasis. Following neural injury the protein kinase-like ER kinase (PERK)-mediated UPR is considered the acute phase adaptive arm. This mechanism, along with a link to ER-mediated apoptosis, is displayed with the proposed effects of salubrinal (SAL) included. 


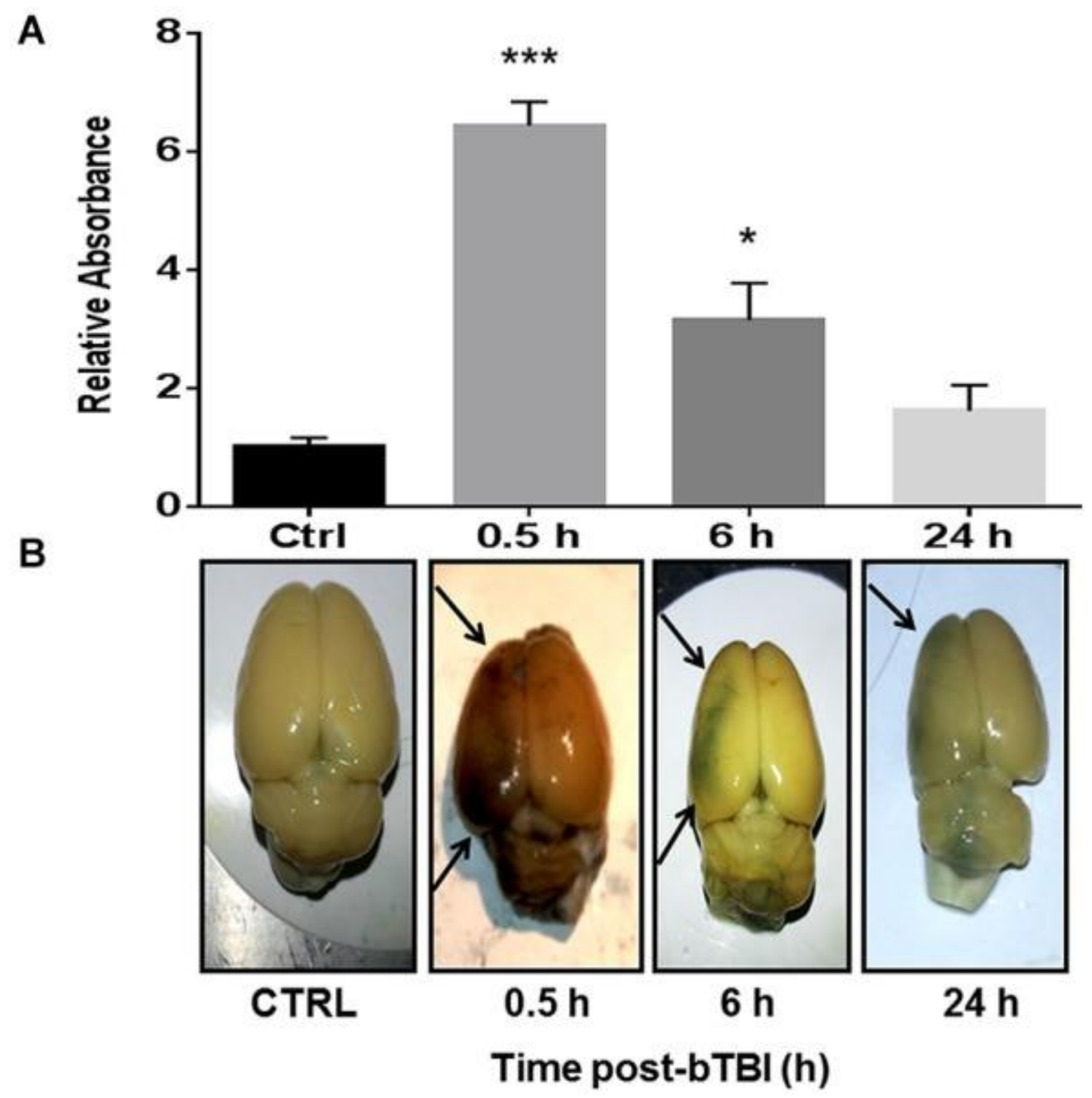

Figure 4.2. Blood brain barrier disruption observed in the contra coup brain after bTBI. (A) Blood Brain Barrier (BBB) disruption as evidenced by increased Evan's Blue absorbance in the left prefrontal cortex (PFC) at $0.5 \mathrm{~h}$ post-bTBI $(* * * p<0.001 \mathrm{vs}$. Ctrl $)$ and $6 \mathrm{~h}$ post-bTBI $\left({ }^{*} p\right.$ $<0.01$ vs. Ctrl) (values represent mean \pm s.e.m.) $(n=4)$. (B) Raw images of Evan's Blue bound albumin in the left brain parenchyma at acute time points post-bTBI. 
A

C
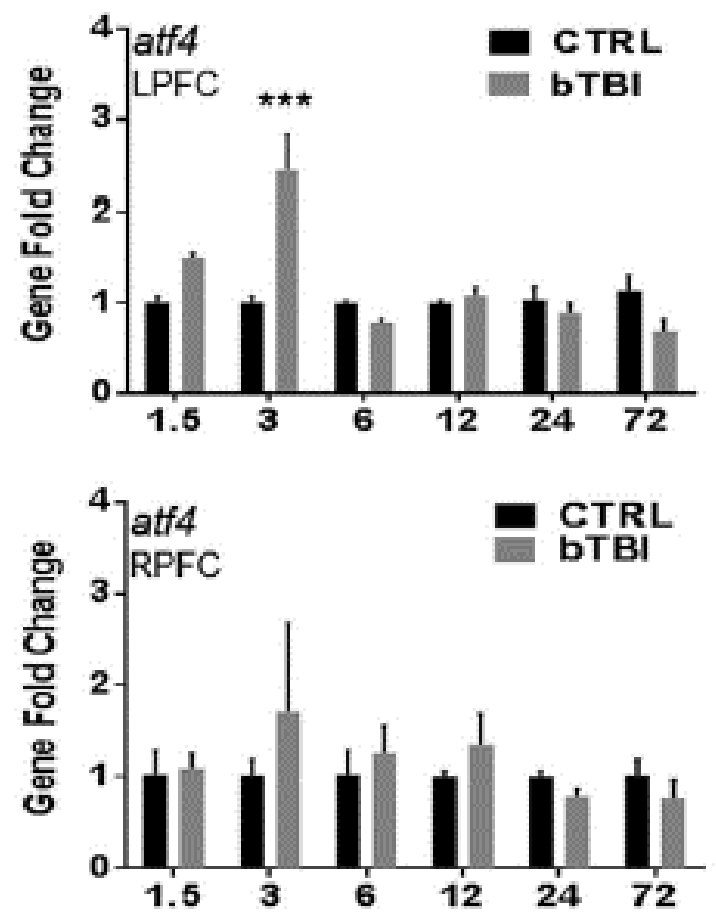

E

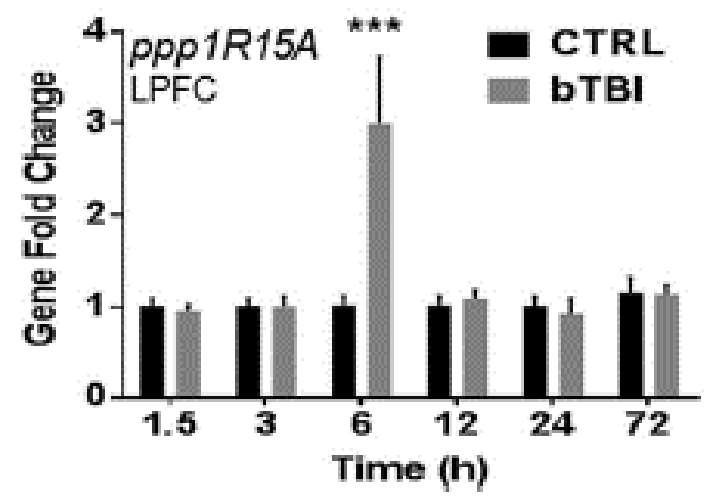

B

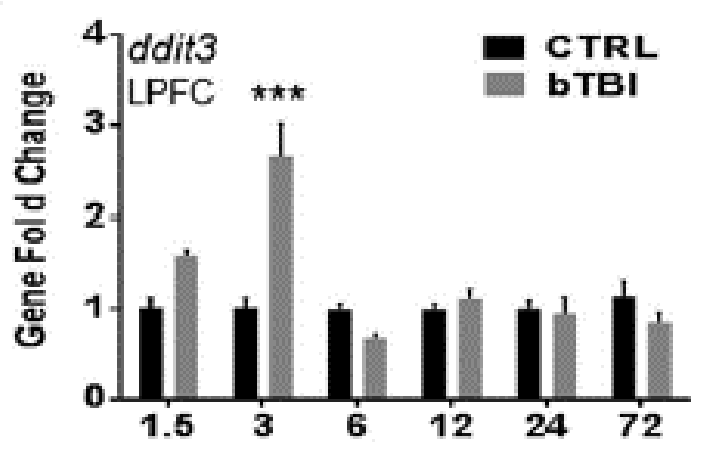

D

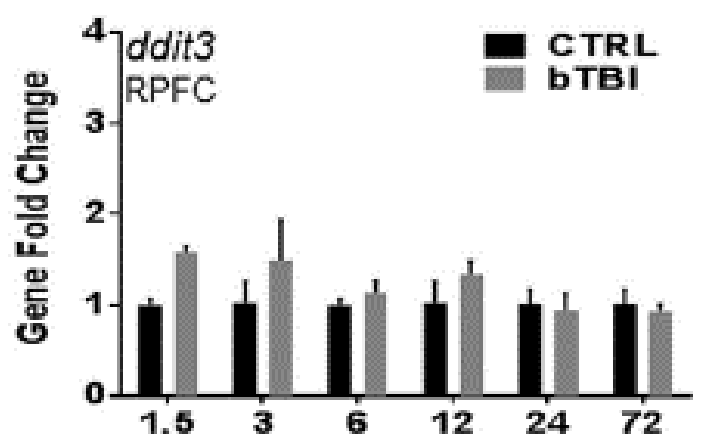

$\mathbf{F}$

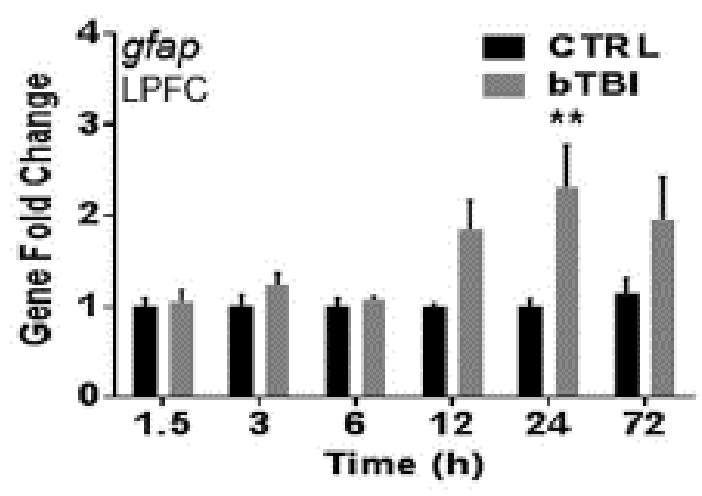

Figure 4.3. Blast exposure upregulates stress response genes atf4, ddit3, ppp1R15A and gfap. Real-time quantitative PCR time course showed a significant increase in mRNA abundance in the left PFC post-bTBI of stress response genes (A) atf4 (activating transcription factor 4) (ATF4) at $3 \mathrm{~h}(* * * p<0.001$ vs. Ctrl at $3 \mathrm{~h})$ and (B) ddit3 (CHOP) at $3 \mathrm{~h}(* * * p<0.001 \mathrm{vs}$. Ctrl at $3 \mathrm{~h}$ ). No real differences were observed in mNRA abundance in the right PFC for (C) atf4 ( $p>$ $0.05)$ and (D) ddit3 ( $p>0.05)$. A significant increase in mRNA abundance was also observed in the left PFC for UPR gene (E) ppplR15A (GADD34) at $6 \mathrm{~h}(* * * p<0.001 \mathrm{vs}$. Ctrl at $6 \mathrm{~h})$, and astrocyte activation marker (F) gfap (GFAP) at $24 \mathrm{~h}(* * p<0.01 \mathrm{vs.} \mathrm{Ctrl}$ at $24 \mathrm{~h})$ (values represent mean \pm s.e.m.) $(n=4)$. 
A

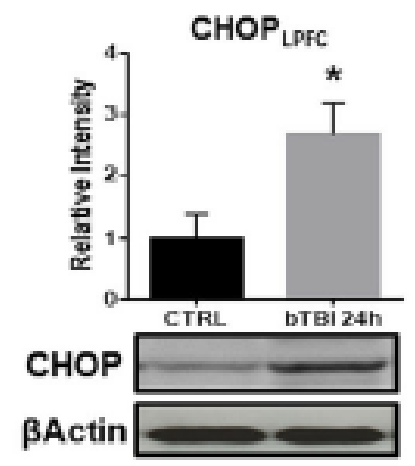

B

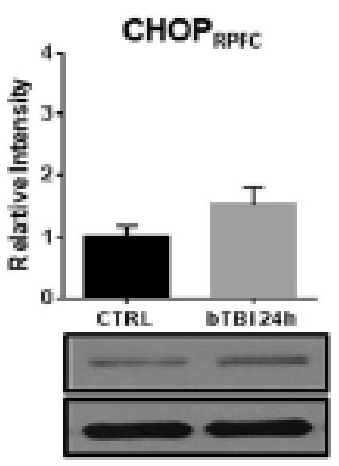

D

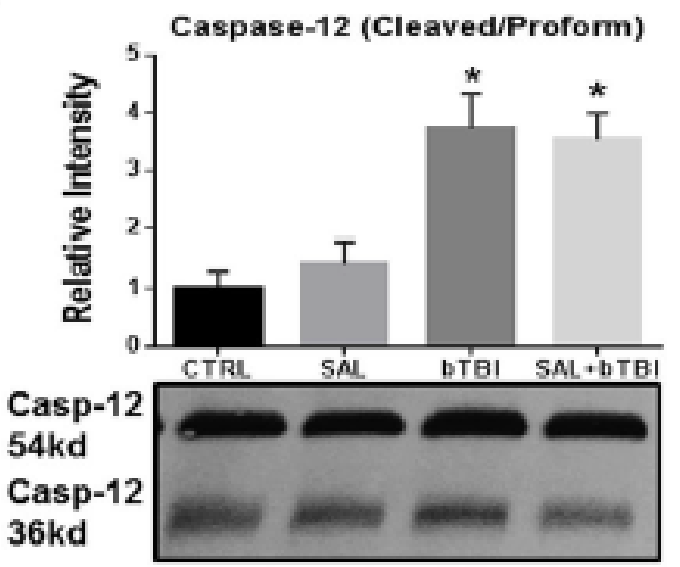

E

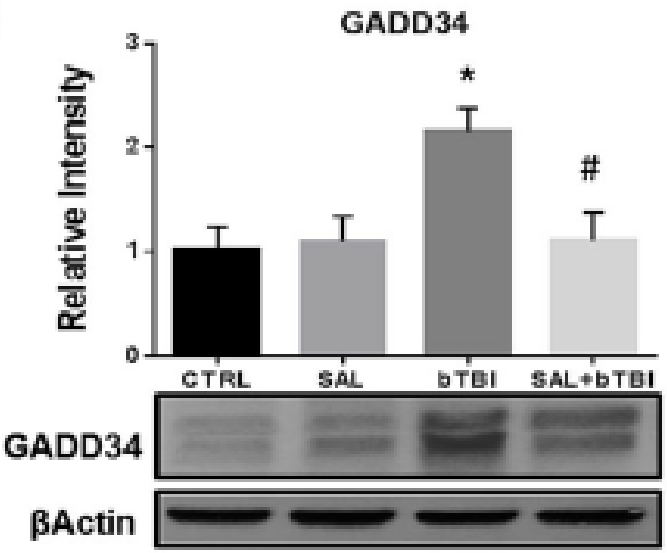

Figure 4.4. SAL attenuates ER stress markers in the contra coup PFC after bTBI (A) Immunoblots show a significant increase in CHOP expression at $24 \mathrm{~h}$ post-bTBI in the left PFC $\left({ }^{*} p<0.05\right.$ vs. Ctrl). (B) No significant differences were observed in the right PFC $(p>0.05$ vs. Ctrl). (C) CHOP expression was significantly increased at $24 \mathrm{~h}$ post-bTBI compared to SAL administered alone $(* p<0.05$ vs. SAL). SAL administration prior to blast exposure attenuated CHOP expression (\#p < 0.05 vs. bTBI). (D) A significant increase in Caspase-12 cleavage was observed at $24 \mathrm{~h}$ in bTBI rats $\left({ }^{*} p<0.05\right.$ vs. SAL), as well as in SAL+bTBI rats at $24 \mathrm{~h}\left({ }^{*} p<\right.$ 0.05 vs. SAL). (E) GADD34 protein expression was significantly increased at $24 \mathrm{~h}$ post-bTBI $\left({ }^{*} p<0.05\right.$ vs. SAL). SAL administration prior to blast exposure attenuated GADD34 protein expression (\#p<0.05 vs. bTBI) (values represent mean \pm s.e.m.; normalized to $\beta$-actin) $(n=4$ $5)$. 

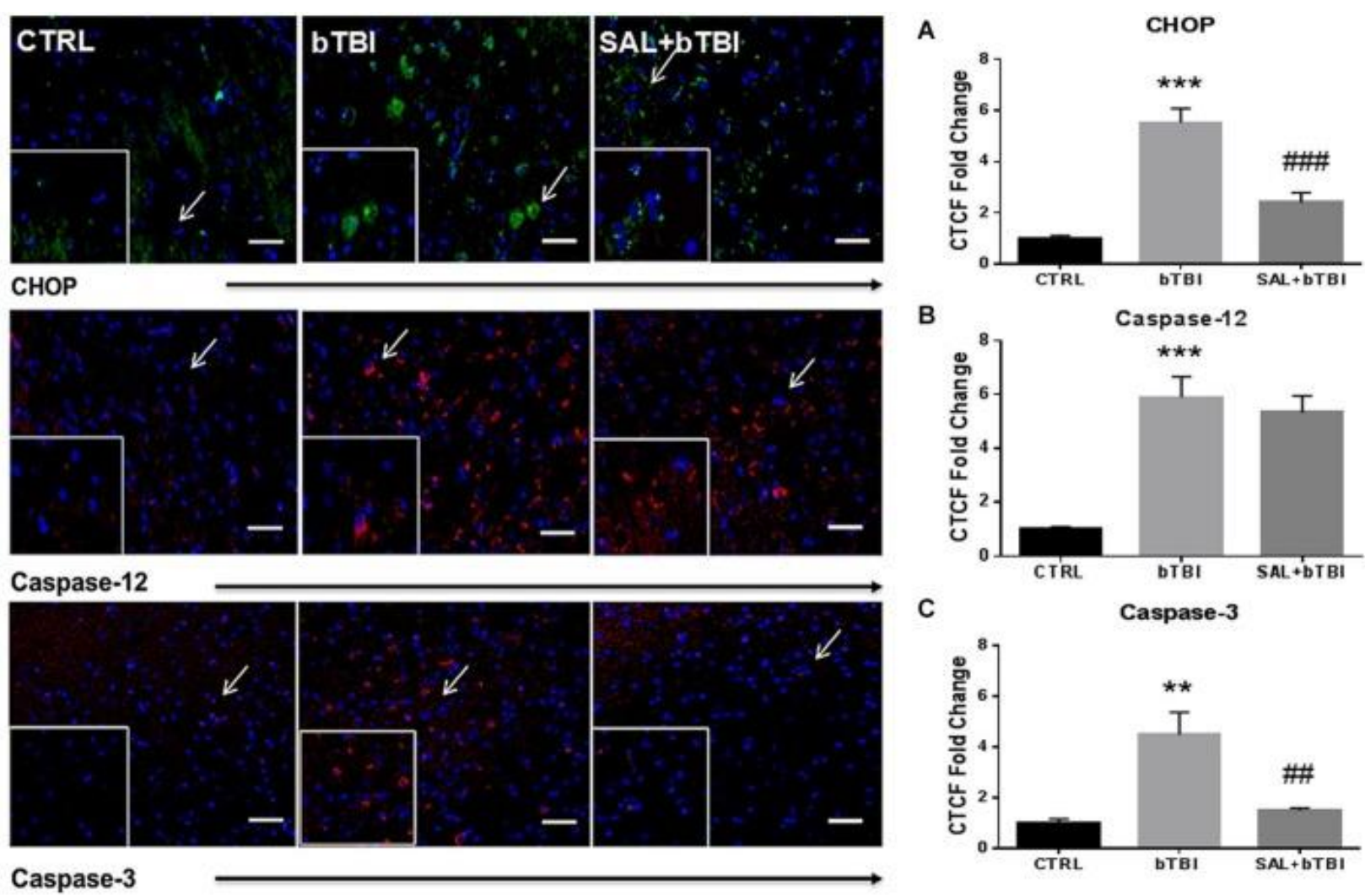

Figure 4.5. SAL modulates fluorescence of apoptosis markers after bTBI. Column of panels display immunofluorescence of apoptosis markers: CHOP (green), Caspase-12 (red) and Caspase-3 (red) (top to bottom). Row of Panels are separated by experimental group: CTRL, bTBI and SAL+bTBI (left to right). All images are from the left PFC region and display the blue nuclear counterstain 4',6-diamidino-2-phenylindole (DAPI). Images are displayed at 20x; arrows demarcate insets at 40x. (Scale bars $=50 \mu \mathrm{m})$. (A) Immunohistochemistry (IHC) shows bTBI augmented CHOP fluorescence in the left PFC at $24 \mathrm{~h}(* * * p<0.001$ vs. Ctrl). SAL administration prior to bTBI attenuated CHOP fluorescence at $24 \mathrm{~h}$ (\#\#\#p<0.001 vs. $24 \mathrm{~h})$ (B) IHC shows bTBI increased Caspase-12 fluorescence in the left PFC at $24 \mathrm{~h}(* * * p<0.001 \mathrm{vs}$. Ctrl). SAL+bTBI rats also displayed increased Caspase-12 fluorescence at $24 \mathrm{~h}(* * * p<0.001$ vs. Ctrl) (C) IHC also shows bTBI augmented Caspase-3 fluorescence in the left PFC at $24 \mathrm{~h}$ $(* * * p<0.001$ vs. Ctrl). SAL administration prior to bTBI mitigated Caspase-3 fluorescence at $24 \mathrm{~h}(\# \# p<0.01$ vs. $24 \mathrm{~h})$ (values represent mean \pm s.e.m.) $(n=4 ; 12$ randomly selected areas from left PFC). 

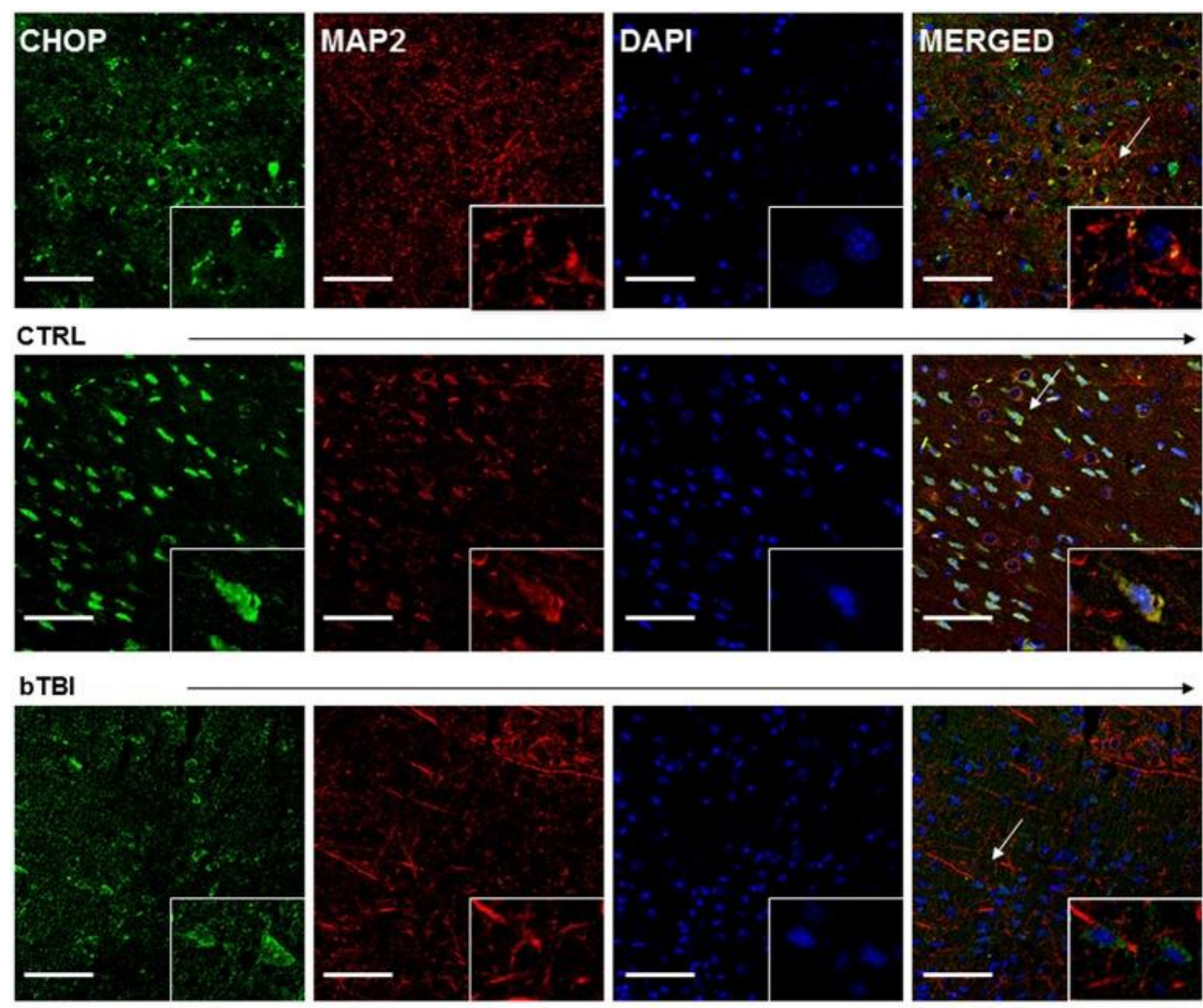

SAL+bTBI

Figure 4.6. SAL reduces CHOP and MAP2 colocalization after blast exposure. Column of panels display immunofluorescence of UPR marker CHOP (green), neuron-specific marker MAP2 (red), nuclear counterstain DAPI (blue), and those three markers merged (yellow) (left to right). Row of panels are separated by experimental group: CTRL, bTBI and SAL+bTBI (top to bottom). All images are from the left PFC region with colocalization determined by levels of yellow in merged images. Images are displayed at 20x; arrows demarcate insets at 40x. (Scale bars $=50 \mu \mathrm{m})$. 

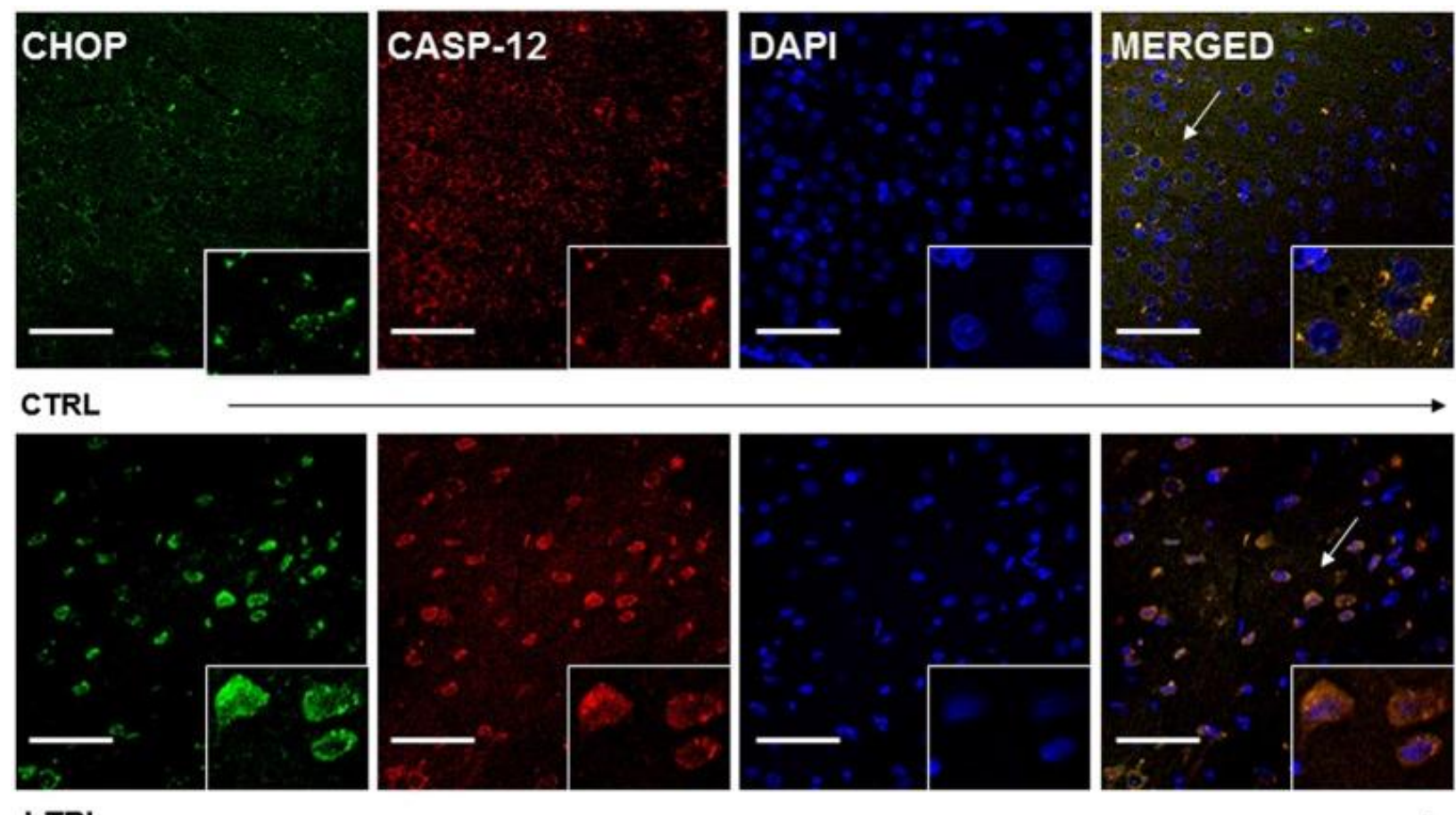

bTBI
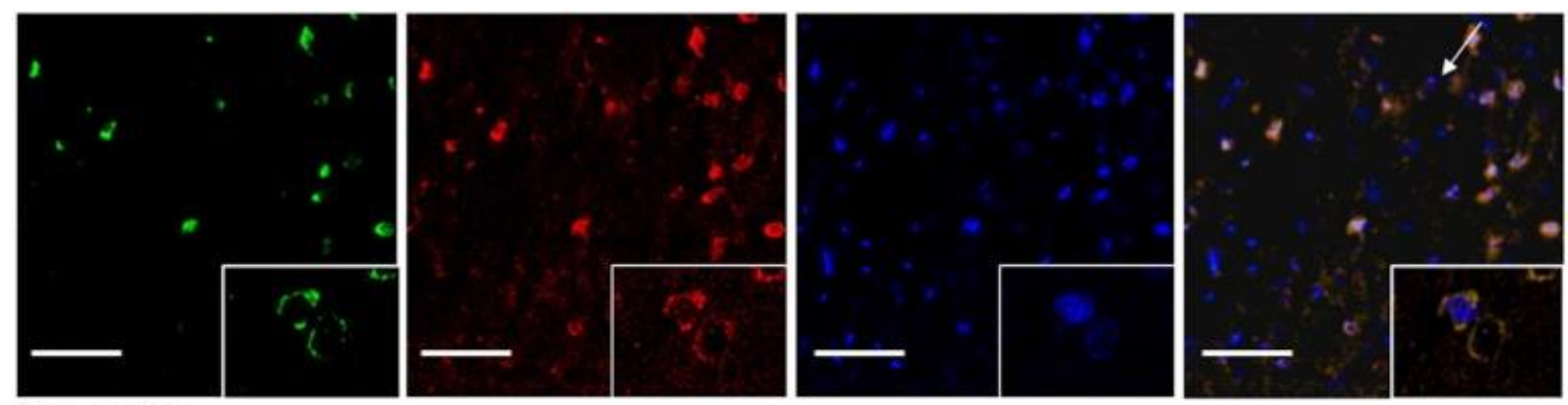

SAL+bTBI

Figure 4.7. CHOP and Caspase-12 colocalize regardless of SAL administration prior to bTBI. Column of panels display immunofluorescence of UPR marker CHOP (green), Caspase12 (red), nuclear counterstain DAPI (blue), and those three markers merged (yellow) (left to right). Row of panels are separated by experimental group: CTRL, bTBI and SAL+bTBI (top to bottom). All images are from the left PFC region with colocalization determined by levels of yellow in merged images. Images are displayed at 20x; arrows demarcate insets at 40x. (Scale bars $=50 \mu \mathrm{m})$. 


\section{A}

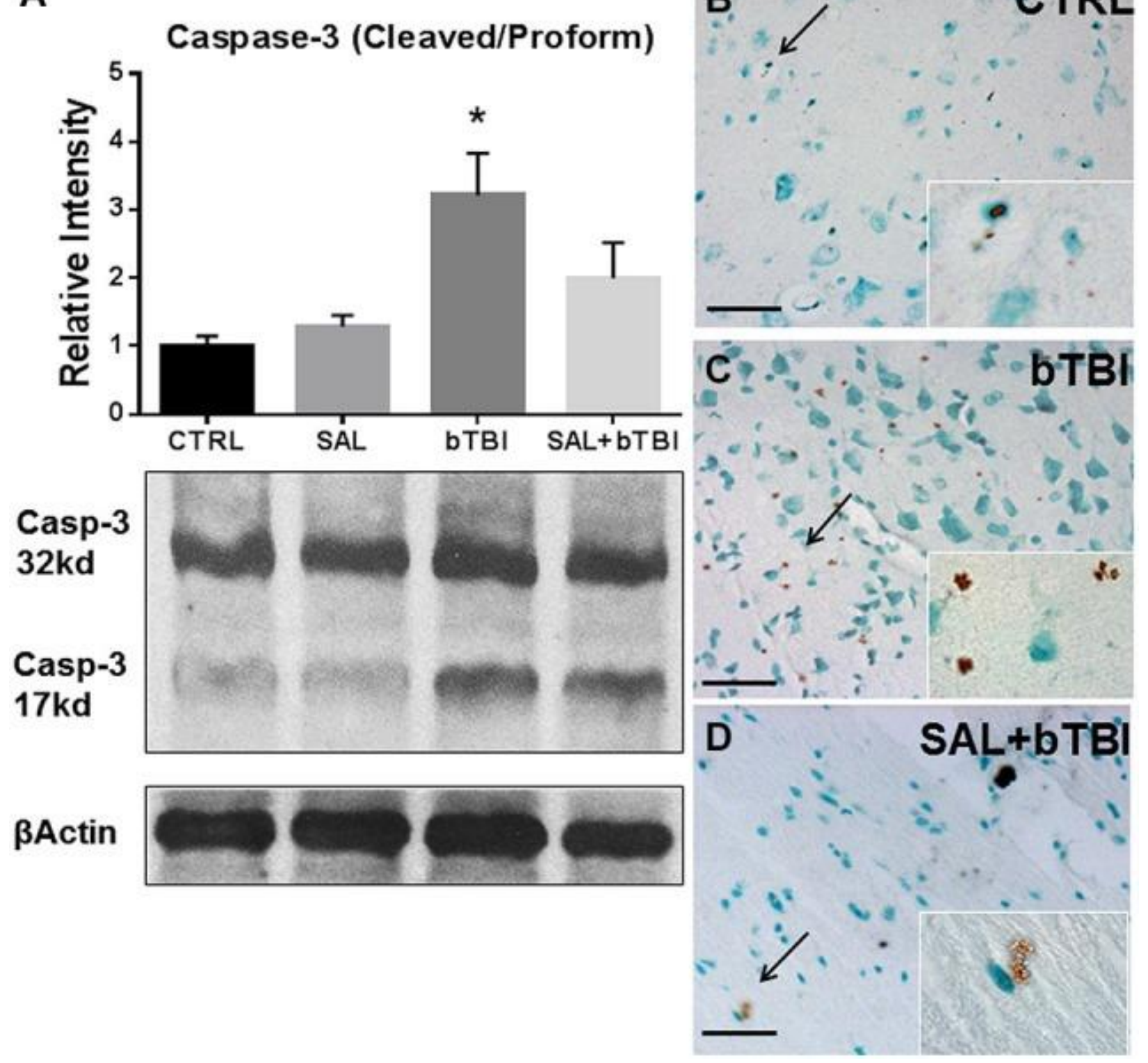

Figure 4.8. SAL reduces apoptosis in the left PFC following blast exposure. (A) Caspase-3 cleavage increased in the left PFC of bTBI rats at $24 \mathrm{~h}\left({ }^{*} p<0.05 \mathrm{vs}\right.$. SAL), but was not increased in SAL+bTBI rats at $24 \mathrm{~h}(p>0.05$ vs. SAL) (values represent mean \pm s.e.m.; normalized to $\beta$-actin) $(n=5)$. (B,C) Evidence of increased cell membrane blebbing, an indicator of early apoptosis, was observed at $24 \mathrm{~h}$ in the left PFC of bTBI rats compared to control rats. (D) Membrane blebbing was reduced in the left PFC of SAL+bTBI rats. Images displayed at 20x; arrows demarcate insets at 63x. (Scale bars $=30 \mu \mathrm{m})(n=4)$. 
A

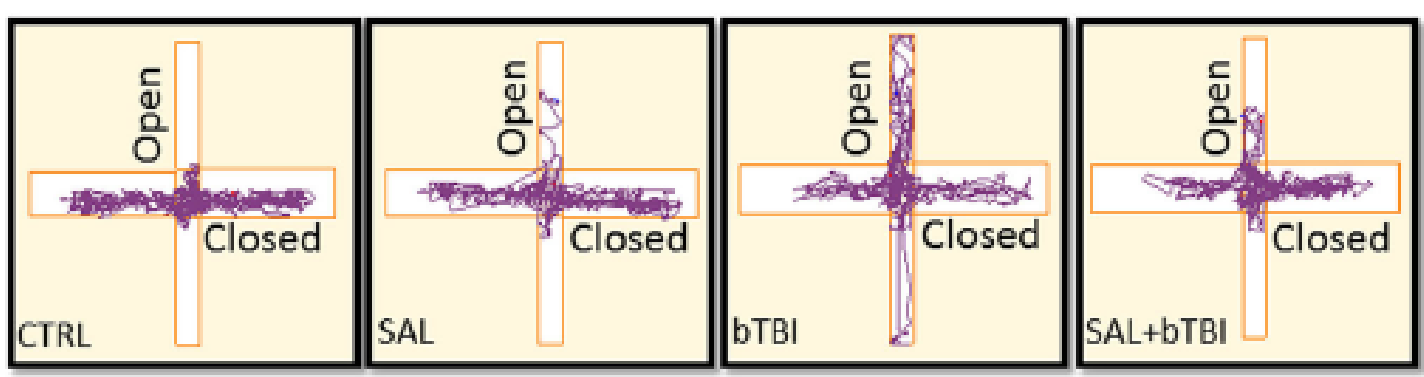

B

Percent Time in Open Arm
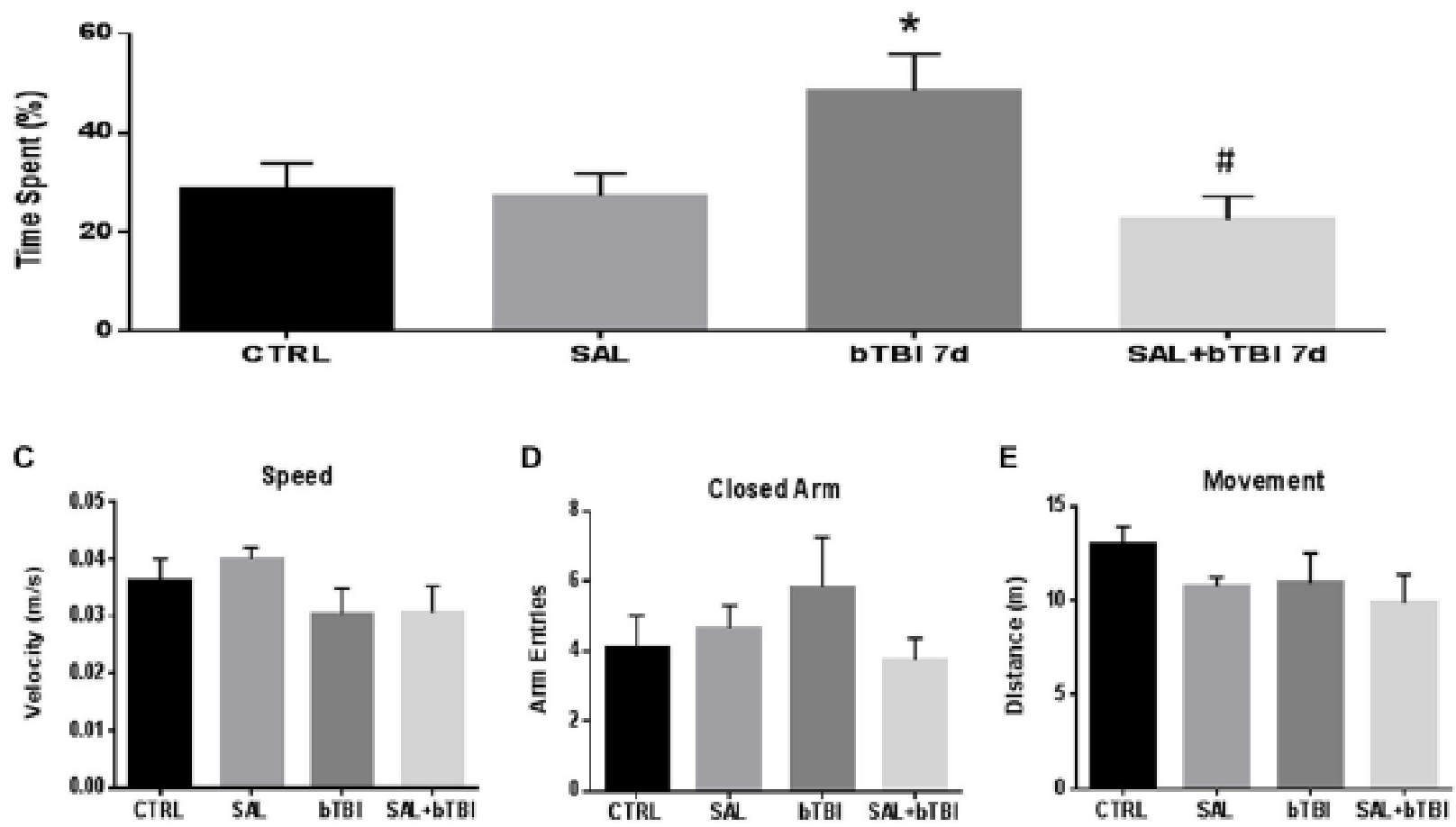

Figure 4.9. SAL ameliorates impulsive-like behavior indicative of PFC damage after Blast. (A) A motion capture track plot of one animal from each experimental group during a single elevated plus maze (EPM) trial using AnyMaze Software ${ }^{\mathrm{TM}}$. (B) Percentage of time spent in the open arms of the EPM was significantly increased in bTBI rats compared to SAL only rats at $7 \mathrm{~d}$ $(* p<0.05$ vs. SAL). SAL administration prior to bTBI decreased the percent time spent in the open arms of the EPM (\#p < 0.05 vs. bTBI) (values represent mean \pm s.e.m.) $(n=12)$. No significant differences were observed between groups for (C) Speed, (D) Closed-Arm Entries, or (E) Movement within the EPM trials (5 min). 


\section{Chapter Five}

Salubrinal reduces oxidative stress, neuroinflammation and impulsive-like behavior in a rodent model of traumatic brain injury

This work has been submitted for publication in Brain Research (2016) 


\begin{abstract}
Traumatic brain injury is the leading cause of trauma related morbidity in the developed world. TBI has been shown to trigger secondary injury cascades including endoplasmic reticulum stress, oxidative stress, and neuroinflammation. The link between secondary injury cascades and behavioral outcome following neurotrauma is poorly understood and warrants further investigation. Using our validated rodent blast injury model, we examined the secondary injury cascades following single injury and how these effects may contribute to risk-taking behavior after repetitive blast. We targeted these pathways with the cellular stress modulator, salubrinal, and investigated its role in decreasing neuroinflammation and risk-taking behavior after blast injury. Single blast increased multiple markers associated with secondary injury cascades including janus-N-terminal kinase phosphorylation, nuclear factor kappa beta activity, inducible nitric oxide synthase protein expression, pro-inflammatory markers, and superoxide levels at 24 hours post-injury. After repeat blast, we observed elevated neuroinflammation and neurodegeneration, in addition to risk-taking behavior measured on the elevated plus maze. Salubrinal administration attenuated secondary injury cascades acutely, and prevented risktaking behavior following repetitive blast. Overall, these results suggest a link between secondary injury cascades and risk-taking behavior that can be modulated by salubrinal administration. Overlapping components of cellular stress were targeted by salubrinal and provide a novel approach to treat neuroinflammation and neuropsychiatric symptoms following neurotrauma.
\end{abstract}




\section{INTRODUCTION}

An estimated 3.2 million Americans are currently living with disabilities from traumatic brain injury (TBI) (Zaloshnja et al., 2008). Risk-taking behavior is one of the most common neuropsychiatric symptoms associated with brain injury and results from damage to the frontal cortex (Schwarzbold et al., 2010; Adhikari et al., 2011; Logsdon et al., 2014; Michael et al., 2015). Risk-taking behavior is a key finding in patients diagnosed with chronic traumatic encephalopathy (CTE) and presents early in disease progression (Banks et al., 2014; Rebetez et al., 2015). CTE is a progressive tauopathy that develops in patients with a history of neurotrauma. The diagnosis of CTE is increasing but prospective studies are limited (Stein et al., 2014). Currently, no therapies for risk-taking behavior are available for patients diagnosed with progressive neurodegenerative disease.

The underlying mechanistic causes linking neurotrauma to subacute neuropsychiatric symptoms are still poorly understood (Lucke-Wold et al., 2014b). Neuroinflammation has emerged as a possible contributory factor to behavior change (Faden et al., 2015). Preclinical models have shown that TBI is associated with a significant inflammatory burden (Kumar et al., 2014). Furthermore, it has been shown that neuroinflammation can persist years after injury in the brains of retired athletes (Coughlin et al., 2014). Recent clinical evidence ties neuroinflammation to neurobehavioral dysfunction (Cho et al., 2013; Wu et al., 2014). We propose that by targeting neuroinflammation, we can possibly improve behavior after TBI. In this paper, we investigate how cellular stress modulation may contribute to changes in the inflammatory burden and neurobehavioral dysfunction in a rodent model of blast TBI.

The endoplasmic reticulum (ER) stress mechanisms have been shown to play a significant role in acute and chronic disease pathology following TBI (Zhang et al., 2012; Abdul- 
Muneer et al., 2014; Begum et al., 2014; Lucke-Wold et al., 2015b). We recently showed that markers of ER stress were increased in the brains of athletes diagnosed with CTE (Lucke-Wold et al., 2015c). Janus-N-terminal kinase (JNK) is a common downstream component of ER stress (Urano et al., 2000), which is activated following TBI (Otani et al., 2002; SzmydyngerChodobska et al., 2010). JNK is also a known tau kinase (Tran et al., 2012). Simultaneous to ER stress activation, oxidative stress occurs and generates free radicals, which play a role in cell death and disease pathology following TBI (Toklu and Tumer, 2015). Free radicals damage cellular membranes, increase carbonyl formation, and can contribute to cell death and neurobehavioral dysfunction (Ferguson et al., 2010).

ER stress may also cause a direct increase in oxidative stress acutely following injury due to accumulated unfolded proteins (Chaudhari et al., 2014). ER stress and oxidative stress have both been associated with increased neuroinflammation after TBI (Deslauriers et al., 2011; Bellezza et al., 2014; Hayashi, 2015). Integral to the process of neuroinflammation is activation

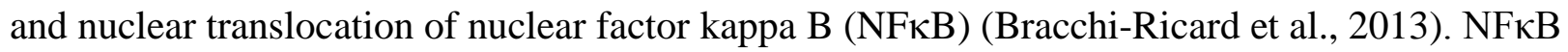
is known to promote pro-inflammatory cytokines, such as, tumor necrosis factor alpha (TNF $\alpha$ ) and interleukin 1 beta (IL-1 $\beta$ ) (Algattas and Huang, 2014). These inflammatory cytokines can signal changes that damage neuronal cell membranes and intracellular organelles (Abdullah and Bayraktutan, 2014). NFkB activity is also known to promote inducible nitric oxide synthase (iNOS) (Algattas and Huang, 2014), which may, under persistent conditions, contribute to neurodegeneration and neurobehavioral dysfunction (Jayakumar et al., 2014). Therefore, neuroinflammation may be a promising target for improving TBI outcome.

Salubrinal (SAL) is a modulator of cellular stress known to inhibit protein phosphatase 1 (Boyce et al., 2005). SAL has been shown to be neuroprotective in models of stroke (Nakka et 
al., 2010) and TBI (Logsdon et al., 2014; Dash et al., 2015). In the present study, we investigated the effects of SAL on oxidative stress and neurodegeneration following blast exposure. We also measured SAL's effects on neuroinflammation in the frontal cortex. Moreover, we examined whether SAL ameliorated risk-taking behavior, a neurobehavioral dysfunction associated with damage to the frontal cortex (Schwarzbold et al., 2010; Adhikari et al., 2011; Logsdon et al., 2014). Modulating intracellular stress may positively influence the extracellular inflammatory milieu leading to improved outcomes following brain injury.

\section{METHODS}

Animals

Fifty-one (51) male Sprague-Dawley rats (Hilltop Lab Animals) at 2-3 months of age were used in this study. Rats were acclimated for 1 week prior to use and housed under 12 hour light/dark conditions with food and water available ad libitum. Animal experiments were performed according to the principles of the Guide for the Care and Use of Laboratory Animals. The West Virginia University Animal Care and Use Committee approved all procedures involving rats.

\section{Salubrinal}

SAL (Tocris Biosciences) was dissolved in 0.5\% DMSO and delivered via intraperitoneal (i.p.) injection at a dose of $1 \mathrm{mg} / \mathrm{kg}$ (Sokka et al., 2007; Liu et al., 2014b).

\section{Blast overpressure exposure}

Blast exposure was induced as previously described (Turner et al., 2013b). Blast pressure with a 0.005 " membrane (15 psi incident wave; 50 psi reflected wave) was determined, from our previous work, to exhibit neural injury markers with no mortality (Turner et al., 2013b; Logsdon 
et al., 2014; Lucke-Wold et al., 2014c). Following blast exposure, rats were returned to a holding cage equipped with a homeothermic heating pad. Once basic reflexes were restored, rats were returned to their home cage.

\section{Experimental groups}

A detailed timeline shows the course of injury and treatment for each group of rats (Figure 5.1). Rats were randomly assigned to one of three treatment groups. Group 1 rats served as sham controls (anesthetized with $4 \%$ isoflurane). Following anesthesia, rats were injected with vehicle (0.5\% DMSO; i.p.) 5 minutes after being placed on blast table. Group 2 rats served as our experimental control group, in which anesthetized rats were oriented similarly to sham rats and subsequently exposed to blast. Rats were injected with vehicle 5 minutes after each blast exposure. Group 3 served as our experimental group. Rats received blast exposure as group 2, but SAL (1 mg/kg; i.p.) was administered 5 minutes after each blast exposure (Rubovitch et al., 2015).

There was two blast TBI subgroupings: a single blast TBI (sTBI) and a repeated blast TBI (rTBI). Rats in the rTBI group received a blast every other day over two weeks, for a total of six blast injuries. This repetitive schedule was determined by our previous work that showed markers of ER stress activation (Lucke-Wold et al., 2015b), and from other TBI models that showed markers of neuroinflammation and neurodegeneration (Mouzon et al., 2012; Mouzon et al., 2014).

Twenty-four (24) sTBI rats were randomly divided into the three treatment groups and euthanized for biochemical analysis at 24 hours post-blast ( $n=4$ rats per group), and immunohistochemistry (IHC) at 72 hours post-blast ( $n=4$ rats per group). Twenty-seven (27) rTBI rats were randomly divided into the three treatment groups and were subject to behavioral 
testing 72 hours after the repetitive blast schedule ( $n=9$ rats per group). Four rTBI rats from each experimental group were euthanized for IHC after behavioral testing was complete.

\section{Tissue preparation}

Rats used for biochemical analysis were euthanized and their brains were rapidly removed in an ice-cold Halt ${ }^{\mathrm{TM}}$ protease/phosphatase inhibitor cocktail mix (Thermo Scientific). The striatum was dissected out from each hemisphere, flash frozen in liquid nitrogen, and stored at $-80^{\circ} \mathrm{C}$ for later measurement of carbonyl and reactive oxygen species (ROS) levels. The frontal cortex was dissected out from each hemisphere, flash frozen in liquid nitrogen and stored at $-80{ }^{\circ} \mathrm{C}$ for later measurement of protein expression and mRNA abundance. Rats used for IHC were anesthetized with $4 \%$ isoflurane and perfused transcardially with ice-cold saline. Following perfusion, brains were removed, flash frozen in isopentane $\left(-60^{\circ} \mathrm{C}\right)$ and stored at $-80^{\circ} \mathrm{C}$ for IHC preparation.

\section{Carbonyl measurement}

For carbonyl measurement, striatal tissue samples from sTBI rats ( $\mathrm{n}=4$ rats per group) were assayed using an OxyBlot Protein Oxidation Detection kit (Millipore). Striatal tissues from each treatment group were sonicated in 6\% sodium docecyl sulfate (SDS). Control samples were mixed with derivation control solution and experimental samples were mixed with 2,4dinitrophenylhydrazine. Samples were incubated for 15 minutes followed by addition of neutralization solution. Samples were loaded (20 ul per well) and electrophoresed on a 10\% acrylamide gel and immunoblotted as outlined in the Western blot methods below.

\section{ROS measurement}

For ROS detection, striatal tissue from sTBI rats were homogenized and cells were isolated by incubation in collagenase at $2 \mathrm{mg} / \mathrm{ml}$ for 30 minutes. Cells were separated by enzyme 
digestion and manual disruption by repeated pipetting. Cells were strained through a 70 um nylon cell strainer followed by centrifugation at $400 \mathrm{x}$ g for 5 minutes. Pellets were resuspended to a concentration of $1.0 \times 10^{6}$ cells/ml in Dulbecco's Modified Eagle Medium (DMEM).

Total ROS and superoxide levels were detected using a Total ROS/Superoxide Detection kit (Enzo Life Sciences) according to manufacturer's instructions. In brief, $100 \mu \mathrm{l}$ of suspended cells were added to each well of a dark-sided 96 well plate with a clear bottom. Cells were incubated overnight at $37^{\circ} \mathrm{C}$ in DMEM. The following day, media was removed and $100 \mu 1$ of ROS/Superoxide Detection Solution was added to each well and incubated in the dark for 1 hour. Detection of total ROS (green) and superoxide (red/yellow) fluorescence was detected at an excitation wavelength of 488/520 nm and an emission wavelength of 550/610 nm, respectively. Data were collected using Gen5 software (BioTek). Concentrations were determined based on a known standard curve.

\section{Western blot}

Protein samples from sTBI and rTBI rats were prepared in 1\% SDS and Western blot analysis was performed as previously described (Lucke-Wold et al., 2014c). Primary antibodies (and dilution factors) were rabbit anti-pJNK monoclonal antibody (mAB) (Thr183/Tyr185) (1:500), rabbit anti-binding immunoglobulin protein $(\mathrm{BiP}) \mathrm{mAB}(1: 1000)$, rabbit anti-JNK mAB (1:1000) (Cell Signaling); mouse anti-NFkB p65 polyclonal antibody (pAB) (1:200), rabbit antiiNOS pAB (1:200) (Santa Cruz). A rabbit anti- $\beta$-actin mAB $(1: 10,000)$ (Cell Signaling) was used as an endogenous control to normalize protein loading. Secondary antibodies were IRDye® 800CW (goat anti-rabbit) and IRDye ${ }^{\circledR}$ 680RD (goat anti-mouse) (LI-COR Biosciences). Images were collected using the Odyssey Classic Infrared Imaging System (LICOR Biosciences). Images were converted to gray scale, and detected bands were quantified 
using Image Studio Lite Software (LI-COR Biosciences). Bands were normalized to $\beta$-actin values to measure relative intensity.

\section{Quantitative real-time polymerase chain reaction}

RNA samples from sTBI and rTBI rats were prepared in TRIzol@ reagent (Life Technologies) and confirmed for quality (1.8-2.1 absorbance ratio). Reverse transcription was conducted and real-time PCR analyses were performed on cDNA using the following oligonucleotide primer sets: TNF $\alpha$ (Rn01525859_g1), IL-1 $\beta$ (Rn00580432_m1), and 18s rRNA (Hs99999901_s1; endogenous control) (Life Technologies). Changes in mRNA abundance were determined by the $\Delta \Delta \mathrm{Ct}$ method with a threshold cycle value of 0.2 normalized to $18 \mathrm{~s}$ rRNA. Histology

Whole brains from sTBI and rTBI rats were mounted on a Leica CM3050S cryostat (Leica Microsystems) set to $-20^{\circ} \mathrm{C}$. Coronal sections of the frontal cortex were sliced at a thickness of $20 \mu \mathrm{m}$ and mounted onto glass slides for IHC staining as previously described (Lucke-Wold et al., 2015b). Briefly, brain slices were circumscribed, and incubated overnight with primary antibodies: iNOS (Santa Cruz), glial fibrillary acidic protein (GFAP), C/E homology protein (CHOP), BiP, and pJNK (Cell Signaling). The next day, an Alexa Flour® secondary antibody (Invitrogen) was applied to slides for 3 hours, and coverslip mounted with Vectashield® 4',6-diamidino-2-phenylindole (DAPI) nuclear counterstain (Vector) . If staining for colocalization, a second set of primary and secondary antibodies were applied prior to fixing the coverslip. Fluorojade B (FJB) (Millipore), and ROS (Enzo Life Sciences) staining was performed in accordance with manufacturer's instructions.

Fluorescent imaging was performed using a Zeiss Axio Observer Z1. For fluorescent staining, 10 cells per slide were randomly selected, outlined, and measured with ImageJ software 
(NIH) by an observer blinded to experimental group. Density was adjusted per mean area to give corrected total cell fluorescence normalized to background. Co-localization quantification with the Just Another Co-localization plugin for ImageJ was used to determine overlap coefficient or Pearson's coefficient. Overlap coefficient was calculated using $\mathrm{k}^{\wedge} 2=\mathrm{k} 1 * \mathrm{k} 2$ with values adjusted to threshold.

\section{Elevated plus maze}

Risk-taking behavior was assessed in rTBI rats using the Elevated plus maze (EPM). The EPM was set at a height of $60 \mathrm{~cm}$ above the floor. The two open arms intersected perpendicular to the two closed arms. Each arm was $50 \mathrm{~cm}$ x $10 \mathrm{~cm}$. The closed arms were encased by black siding $30 \mathrm{~cm}$ tall. Each rat was placed in the middle of the EPM facing an open arm and tracking was performed for 5 minutes using AnyMaze ${ }^{\mathrm{TM}}$ software (Stoelting), which pinpointed the location of the rat's head and body continuously throughout the testing trial.

\section{Statistical analysis}

Data were analyzed using a one-way analysis of variance (ANOVA) followed by Newman-Keul's post hoc tests for between groups comparison. For colocalization studies, Pearson's coefficient was obtained for control sections. Overlap coefficient was obtained for experimental groups to determine the extent of same-cell protein expression. Sample sizes were determined using a power analysis with an $\alpha$ of 0.05 , a $\beta$ of 0.2 , and a sample effect of 0.4 for behavioral data and 0.3 for all other data (DSS Research Power Analysis). $P<0.05$ was considered significant.

\section{RESULTS}

\section{SAL reduces oxidative stress after sTBI}


Figure 5.2A shows a significant difference in carbonyl levels between groups after sTBI $\left(\mathrm{F}_{(2,9)}=10.21 ; P<0.01\right)$. A significant increase in carbonyl levels was measured in sTBI rats as compared to control rats $(q=6.26 ; P<0.01)$. SAL administration significantly reduced carbonyl levels $(q=4.23 ; P<0.05)$ when compared to vehicle-treated sTBI rats.

Figure 5.2B shows a significant difference in superoxide levels between experimental groups after sTBI $\left(\mathrm{F}_{(2,9)}=7.68 ; P<0.05\right)$. A significant increase in superoxide levels was measured in STBI rats when compared to control rats $(q=4.92, P<0.05)$. SAL administration significantly reduced superoxide levels as compared to vehicle-treated sTBI rats $(q=4.68, P<$ 0.01). Figure 5.2C shows no difference in total ROS levels $\left(\mathrm{F}_{(2,9)}=0.16 ; P>0.05\right)$.

\section{SAL attenuates neurodegeneration after STBI}

Figure 5.3A shows a significant difference in corrected total cell fluorescence for GFAP at 72 hours post-sTBI $\left(\mathrm{F}_{(2,29)}=35.23 ; P<0.001\right)$. A significant increase in GFAP fluorescence was observed in sTBI rats compared to control rats $(q=10.07, P<0.001)$. SAL administration significantly reduced GFAP fluorescence when compared to vehicle-treated sTBI rats $(q=7.58$, $P<0.001)$

Figure 5.3B shows a significant difference in corrected total cell fluorescence for FJB at 72 hours post-sTBI $\left(\mathrm{F}_{(2,29)}=7.43 ; P<0.01\right)$. A significant increase in FJB fluorescence was observed in sTBI rats compared to control rats $(q=5.18, P<0.05)$. SAL administration significantly reduced FJB fluorescence when compared to vehicle-treated sTBI rats $(q=3.32 ; P$ $<0.05)$.

\section{SAL mitigates neuroinflammation after sTBI}

Figure 5.4A indicates a significant difference between experimental groups in JNK phosphorylation after sTBI $\left(\mathrm{F}_{(2,9)}=9.04 ; P<0.01\right)$. A significant increase in JNK 
phosphorylation at 24 hours was observed in sTBI rats as compared to control rats $(q=5.79 ; P<$ 0.01). SAL administration significantly attenuated JNK phosphorylation when compared to vehicle-treated sTBI rats $(q=4.31 ; P<0.05)$. No difference in total JNK protein expression was observed at 24 hours post-sTBI $(P>0.05)$.

Figure 5.4B shows no significant difference in BiP expression between experimental groups $\left(\mathrm{F}_{(2,9)}=0.93 ; P>0.05\right)$.

Figure 5.4C shows a significant difference in NFkB p65 expression between experimental groups at 24 hours after sTBI $\left(\mathrm{F}_{(2,9)}=4.67 ; P<0.05\right)$. NFkB p65 expression was significantly increased in sTBI rats compared to control rats $(q=4.101 ; P<0.05)$. SAL administration significantly reduced NFאB p65 expression when compared to vehicle-treated STBI rats $(q=3.24 ; P<0.05)$.

Figure 5.4D shows a significant difference in iNOS expression at 24 hours after sTBI $\left(\mathrm{F}_{(2,9)}=6.81 ; P<0.05\right)$. A significant increase in iNOS expression was measured in sTBI rats compared to control rats $(q=4.92 ; P<0.05)$. SAL administration significantly reduced iNOS expression when compared to vehicle-treated sTBI rats $(q=3.97 ; P<0.05)$.

Figure 5.4E shows a significant difference in IL-1 $\beta$ mRNA abundance at 24 hours postsTBI amongst the treatment groups $\left(\mathrm{F}_{(2,9)}=5.62 ; P<0.05\right)$. A significant increase in IL-1 $\beta$ mRNA abundance was measured in sTBI rats compared to control rats $(q=4.13 ; P<0.05)$. SAL administration significantly reduced IL- $1 \beta$ mRNA abundance when compared to vehicle-treated sTBI rats $(q=4.08 ; P<0.05)$.

Figure 5.4F shows a significant difference amongst the treatment groups in TNF $\alpha$ mRNA abundance at 24 hours post-sTBI $\left(\mathrm{F}_{(2,9)}=5.54 ; P<0.05\right)$. A significant increase in TNF $\alpha$ mRNA abundance was measured in sTBI rats compared to control rats $(q=4.49 ; P<0.05)$. SAL 
administration significantly reduced TNF $\alpha$ mRNA abundance when compared to vehicle-treated sTBI rats $(q=3.47 ; P<0.05)$.

\section{ER stress and neuroinflammation markers colocalize}

Figure 5.5A shows IHC colocalization (yellow) of ER stress marker CHOP (green) with ER stress marker BiP (red). Pearson's coefficient revealed a low correlation in vehicle controls $(r$ $=0.21)$, a high correlation in rTBI + Vehicle $(r=0.82)$, and a low correlation in rTBI + SAL $(r=$ $0.20)$.

Figure 5.5B shows IHC colocalization (yellow) of ER stress marker CHOP (green) with ER stress marker pJNK (red). Overlap Pearson's coefficient revealed no correlation in vehicle controls $(r=0.00)$, a medium correlation in rTBI + Vehicle $(r=0.61)$, and no correlation in rTBI $+\operatorname{SAL}(r=0.00)$.

\section{SAL attenuates neuroinflammation after rTBI}

Figure 5.6A shows IHC colocalization (yellow) of ER stress marker CHOP (green) with neuroinflamation marker iNOS (red). Overlap Pearson's coefficient revealed a low correlation in vehicle controls $(r=0.17)$, a medium correlation in rTBI + Vehicle $(r=0.65)$, and a low correlation in $\mathrm{rTBI}+\mathrm{SAL}(r=0.22)$.

Figure 5.6B shows a significant difference in corrected total cell fluorescence for GFAP at 72 hours post-rTBI $\left(\mathrm{F}_{(2,29)}=6.62 ; P<0.01\right)$. A significant increase in GFAP fluorescence was observed in rTBI rats compared to vehicle-control rats $(q=4.69, P<0.01)$. A significant increase in GFAP fluorescence was also observed in rTBI + SAL rats compared to vehiclecontrol rats $(q=4.99, P<0.05)$.

SAL ameliorates risk-taking behavior after rTBI 
Figure 5.7A shows a significant difference in open arm time at two weeks post-rTBI amongst the treatment groups $\left(\mathrm{F}_{(2,24)}=4.72 ; P<0.05\right)$. A significant increase was measured in the time that the rTBI rats spent in the open arms of the EPM compared to control rats $(q=4.09$; $P<0.05)$. Interestingly, SAL administration significantly attenuated the open arm time when compared to vehicle-treated rTBI rats $(q=3.31 ; P<0.05)$. Figure 5.7B shows no difference in the total distance the rats travelled between the experimental groups $\left(\mathrm{F}_{(2,24)}=2.54 ; P>0.05\right)$. Track plots from Anymaze ${ }^{\mathrm{TM}}$ were overlaid for each subject to provide a clear visual representation of the rats' behavior during the EPM trials (Figure 5.7C-E).

\section{Mechanism of blast injury}

Figure 5.8 shows a proposed detailed schematic of the injury cascade following blast injury. We propose blast TBI may cause vascular disruption and blood extravasation into the brain parenchyma can damage plasma membranes and trigger secondary injury cascades (Uranga et al., 2009; Liu et al., 2013; Nisenbaum et al., 2014). The secondary injury cascades may contribute to neurodegeneration and neurobehavioral dysfunction.

\section{DISCUSSION}

Blast TBI models cause a primary injury due, in part, to acceleration-deceleration forces to the rat's head (Goldstein et al., 2012). The current study shows an initiation of secondary injury cascades including oxidative stress and ER stress following primary injury in addition to glial reactivity and neurodegenerative changes. We also show these cellular stress responses occurred concurrent with increases in neuroinflammatory markers after blast TBI.

Neuroinflammation is a common secondary effect of brain injury and has been demonstrated in a variety of rodent TBI models (Abdul-Muneer et al., 2013; Cho et al., 2013; Abdul-Muneer et al., 
2014; Algattas and Huang, 2014; Roth et al., 2014), and has been linked clinically to single and repetitive head injuries (Aungst et al., 2014; Webster et al., 2015). While previous studies have shown an association between ER stress and neuroinflammation, what remains unknown is whether regulation of these responses would affect neurodegeneration and improve neurobehavioral outcome (Fenn et al., 2015). Additionally, ER stress activation has been proposed to directly increase oxidative stress particularly in the striatum (Malhotra and Kaufman, 2007). Using the ER stress modulator SAL, we show a potential link between these pathways. Moreover, how brain injury contributes to neuroinflammation and subsequent neurobehavioral dysfunction has not yet been elucidated. Using SAL as a modulator, we also offer compelling evidence that acute cellular stress cascades, not only closely link to subacute neuroinflammation, but also subsequent behavioral alteration.

An important secondary injury cascade often increased following TBI and intimately linked to ER stress is oxidative stress (Cho et al., 2013). We report an increase in oxidative stress damage in the striatum following blast exposure, as evidenced by increased carbonyl and NADPH-oxidase (NOX)-mediated superoxide levels. Superoxide production is a consequence of TBI mainly through activation of the NOX system (Brennan et al., 2009; Zhang et al., 2012; Loane et al., 2013). A previous report indicated that NOX and superoxide levels were elevated at 6 hours after blast TBI and persisted for 72 hours (Ansari et al., 2014). Additionally, NOX-4 mediated oxidative stress perpetuates damage to neuronal membranes leading to cell death (Zhang et al., 2012). A late-onset symptom of CTE is motor dysfunction similar to Parkinson's disease that indicates cell death and damage to the striatum (Stern et al., 2011). In a future study we will investigate late-onset motor disturbance associated with the striatal damage reported herein. We will further elucidate whether repeat or single injury is necessary for motor deficits. 
Persistent damage to the cell from oxidative stress can trigger a measurable increase in JNK phosphorylation (Quan et al., 2015), and NFאB activity [39, 40], affecting neuroinflammation. An increase in both pJNK and NFאB p65 expression were demonstrated in this study. JNK can signal NFאB translocation to the nucleus, which may upregulate proinflammatory mediators such as iNOS, TNF $\alpha$ and IL-1 (Algattas and Huang, 2014; Ruan et al., 2015). In line with this ensuing injury cascade following blast, we found increased levels of proinflammatory markers: NFאB, iNOS, TNF $\alpha$ and IL-1 $\beta$. These pro-inflammatory markers are thought to cause a sudden oxidative burst that overwhelms antioxidant defense cascades leading to further damage of surrounding brain tissue (Liao et al., 2013). We show repetitive blast TBI causes a subacute increase in iNOS fluorescence, suggesting a persistent inflammatory response. Consequently, while neuroinflammation is a necessary response to brain injury (Finnie, 2013), unabated, persistent neuroinflammation can lead to irreversible neurodegeneration and poor injury outcome.

Furthermore, we recently showed that ER stress is an important secondary injury response activated after blast TBI (Logsdon et al., 2014), and human CTE (Lucke-Wold et al., 2015c). ER stress can also lead to neurobehavioral dysfunction following TBI (Dash et al., 2015; Lucke-Wold et al., 2015b). We show ER stress markers CHOP and pJNK fluorescence to be increased following repetitive injury. Similar to oxidative stress, the ER stress response has significant cross talk with neuroinflammatory pathways through JNK signaling (Prell et al., 2014). A recent study suggests crosstalk between JNK phosphorylation and NFKB activity after inflammatory challenge with an ER stressor (Ruan et al., 2015).

We show, for the first time, that SAL administration post-injury attenuates iNOS protein expression and JNK phosphorylation. This is in agreement with a recent study where SAL 
administration reduced $\mathrm{NF \kappa B}$ and microglia activation in a model of Alzheimer's disease (Huang et al., 2012). SAL also improved chronic motor performance in a model of spinal cord injury (Ohri et al., 2013). In a future study, we will investigate if SAL improves motor performance long-term following TBI. Further investigation is needed to see if acute modulation of cellular stress pathways will lead to long-term behavioral improvements. Docosahexaenoic acid, another less-specific ER stress modulator, improved cognitive performance following TBI (Begum et al., 2013; Lucke-Wold et al., 2015b). Based on the results of this study and the supporting background information, we propose a novel mechanism of action for the beneficial properties of SAL through downstream regulation of NFKB activity. In support of this claim, we demonstrated that SAL administration after blast attenuated pJNK, NFkB, iNOS, TNF $\alpha$, and IL$1 \beta$ expression.

SAL also decreased markers of glial reactivity after single blast exposure, suggesting a protective effect at the blood-brain barrier as previously reported (Logsdon et al., 2014). Surprisingly, SAL had no influence on glial reactivity following repetitive blast exposure, possibly due to a priming effect of persistent neuroinflammation (Ma et al., 2014). The repetitive injury schedule may have reduced the measured glial reactivity at 72 hours compared to a single blast exposure. Results suggest that astrocyte activation may not contribute to the subacute neurobehavioral dysfunction observed with our repetitive TBI model.

Abnormal behavioral symptoms can be a manifestation of persistent neuroinflammation, and are of particular clinical relevance for patients suffering multiple mild head injuries (Sominsky et al., 2015). We showed that rTBI rats displayed risk-taking behavior in the EPM 72 hours following the last blast. Interestingly, SAL administration reduced risk-taking behavior when administered after each blast exposure. 
At 72 hours following the last blast, IHC results suggest that repetitive blast exposure increases markers of ER stress and neuroinflammation in the frontal cortex. The results also suggest SAL administration reduced CHOP expression. The Pearson's coefficient values suggest CHOP colocalized with BiP, pJNK and iNOS in the frontal cortex at 72 hours post-rTBI. Colocalization strengths also suggest SAL may have an effect on ER stress and neuroinflammation within the same cells. These markers have been postulated to cause the neuronal demise responsible for neurobehavioral dysfunction. Consequently, and most importantly, we suggest that SAL may mitigate subacute markers of neuroinflammation and thereby reduce risk-taking behavior mediated by the frontal cortex after repetitive brain trauma.

These findings have broad reaching implications regarding the importance of targeting cellular stress acutely post-injury in order to reduce subacute neuroinflammation and ameliorate neurobehavioral dysfunction. We showed that ER stress modulation after blast injury decreases acute superoxide formation in the striatum, attenuates neuroinflammatory markers in the frontal cortex, and ameliorates sub-acute risk-taking behavior in rats. In doing so, we map a unique interconnection between intracellular stress cascades and neuroinflammation. Further pre-clinical studies are warranted to determine the role of oxidative stress, ER stress and neuroinflammation at subacute time points following brain injury and how these pathways contribute to neurobehavioral dysfunction.

In summary, cellular stress and neuroinflammation are intrinsically interconnected and play an important role in injury progression following TBI. We show that blast exposure induced markers of oxidative stress, which is known to contribute to the exacerbation of ER stress and neuroinflammation. Furthermore, we show that ER stress may be linked neuroinflammation through the JNK-mediated NFאB pathway. Surprisingly, SAL reduced markers of oxidative 
stress and ER stress; thereby, reducing markers of neuroinflammation post-blast. The likely mechanism is reduction of JNK phosphorylation. Most importantly, SAL ameliorated risk-taking behavior when administered after each repetitive blast exposure. Secondary injury modulation with key multi-target pharmaceutics offers a promising approach to reduce the long-term neuropsychiatric symptoms associated with head injuries. 


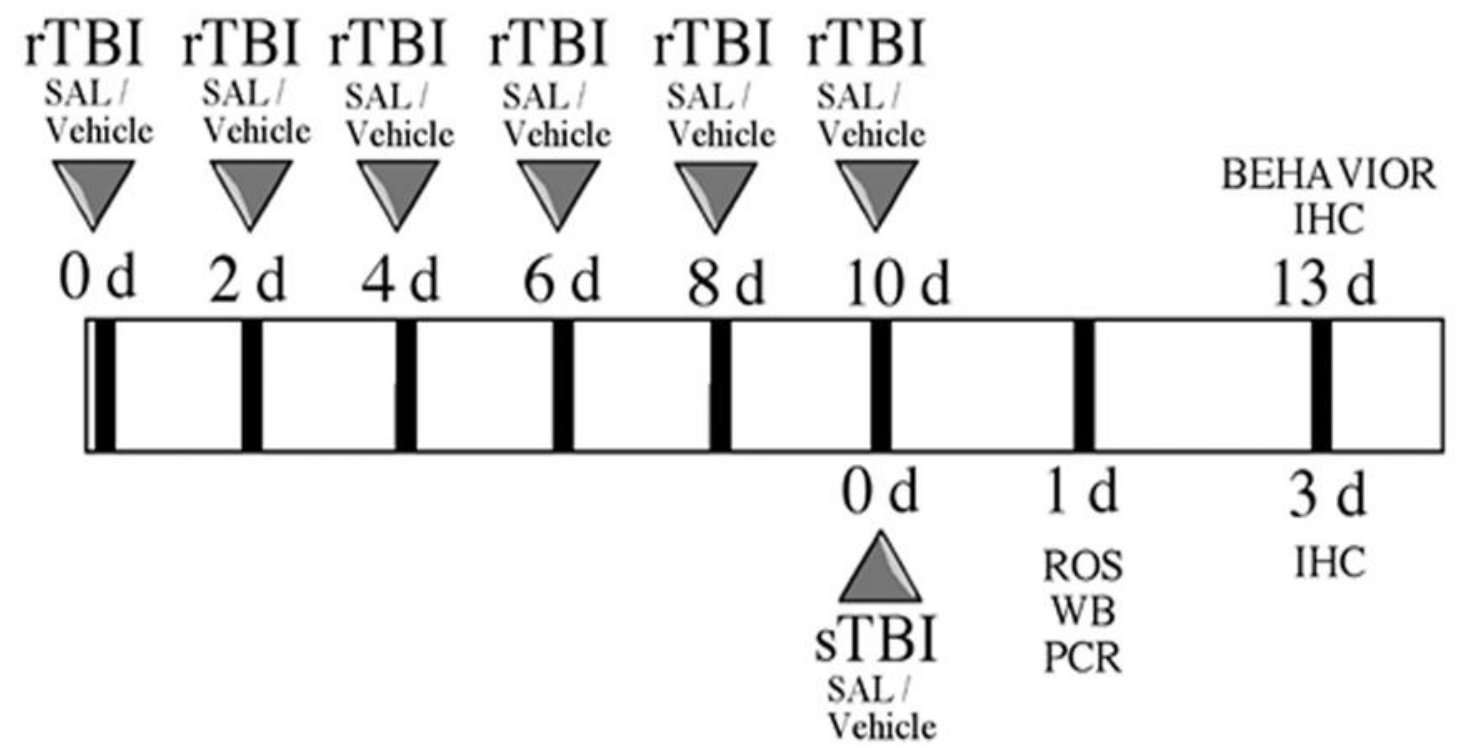

Figure 5.1. Experimental design

Detailed experimental timeline showing: rTBI (repetitive blast) animals at top; sTBI (single blast) animals at bottom. SAL (Salubrinal); ROS (Reactive oxygen species); WB (Western blot); PCR (Polymerase chain reaction); IHC (Immunohistochemistry). 
A

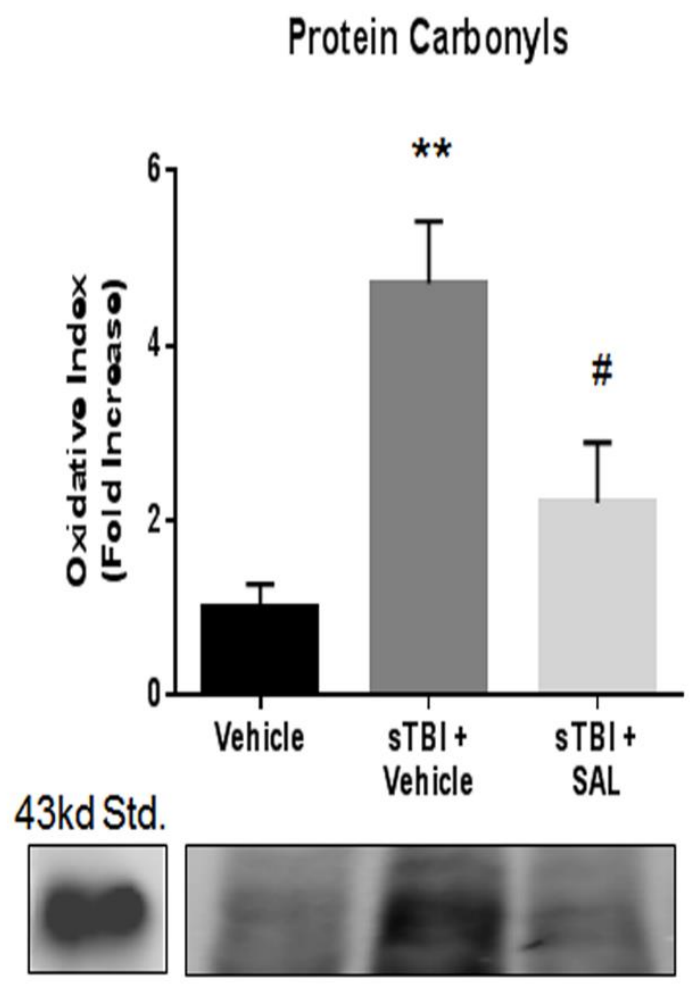

B

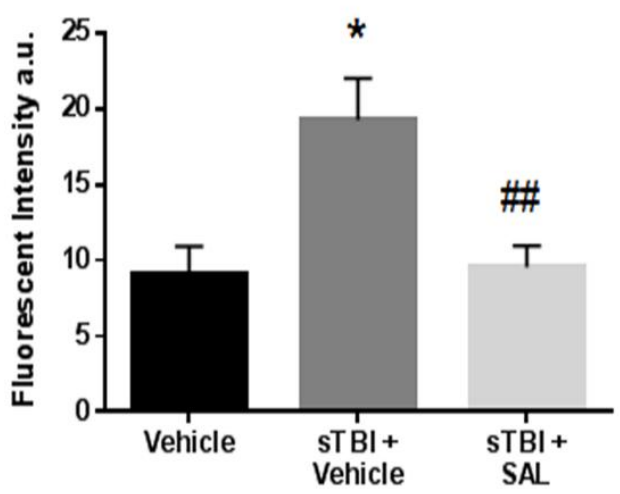

C

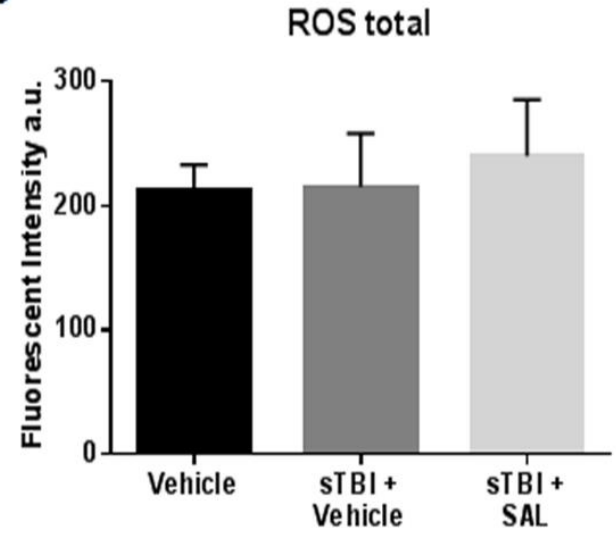

Figure 5.2. SAL reduced markers of oxidative stress after single blast

(A) A significant increase in protein carbonyl levels was measured at 24 hours post-sTBI (**P< 0.01 vs Vehicle); SAL treatment significantly attenuated carbonyl levels ( $\# P<0.01$ vs sTBI). (B) A significant increase in superoxide levels was measured at 24 hours post-sTBI $\left({ }^{*} P<0.05\right.$ vs Vehicle); SAL treatment significantly attenuated superoxide levels (\#\#P<0.01 vs sTBI). (C) No significant differences were observed in total ROS levels $(P>0.05)$. One-way ANOVA, Newman-Keul's post hoc. Mean \pm S.E.M. $n=4$. 

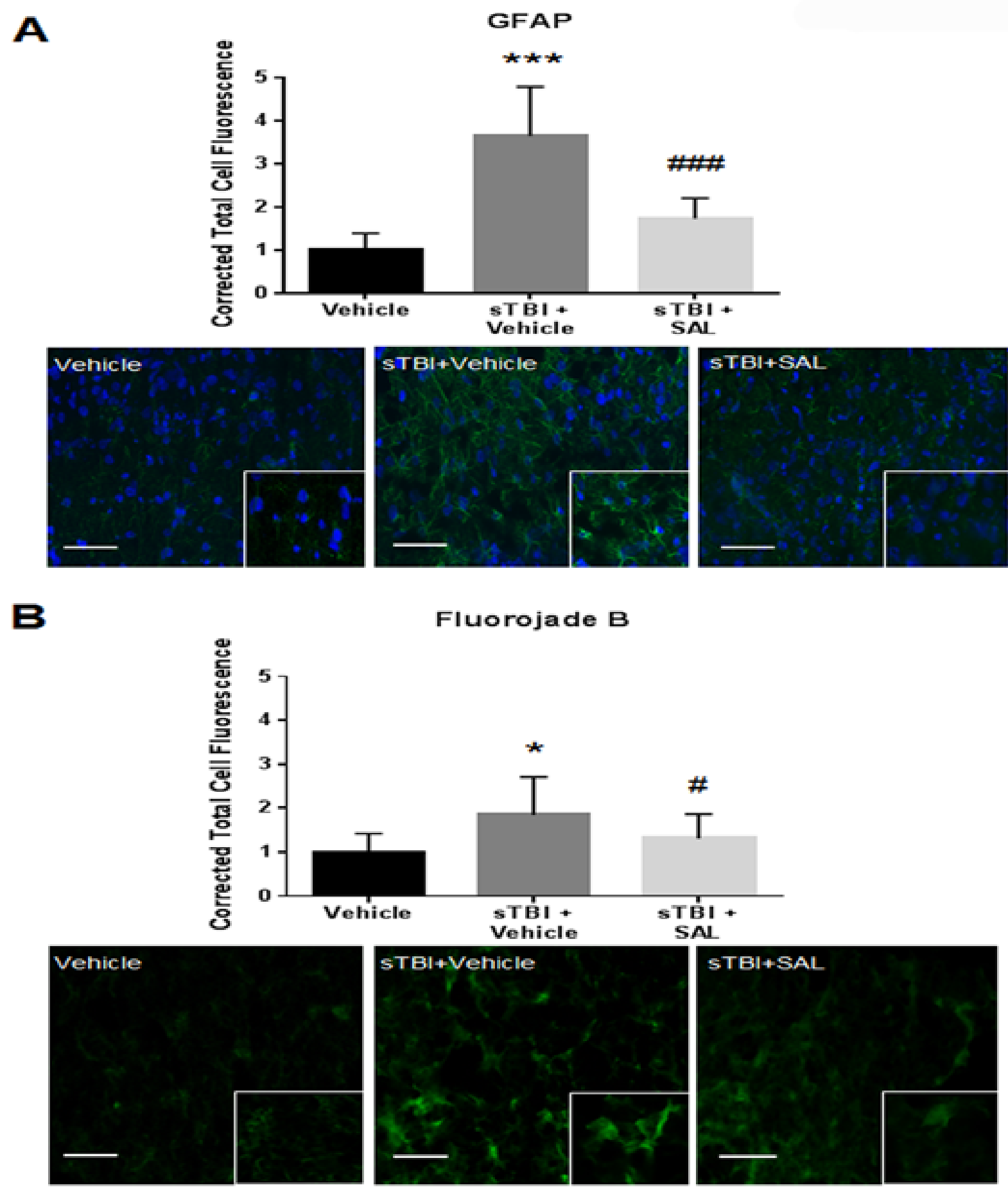

Fluorojade B

Figure 5.3. SAL attenuated markers of neurodegeneration after single blast

(A) We observed a significant increase in GFAP (green) fluorescence at 72 hours post-sTBI $(* * * P<0.001$ vs Vehicle); SAL significantly reduced GFAP fluorescence (\#\#\#P<0.001) $(n=$ 4). GFAP images include nuclear counterstain DAPI (blue). (B) We observed a significant increase in FJB (green) fluorescence at 72 hours post-sTBI ( $P<0.05$ vs Vehicle); SAL significantly reduced FJB fluorescence ( $\# P<0.05)$. One-way ANOVA, Newman-Keul's post hoc. Mean \pm S.E.M. $n=4$. Images are displayed at 20x; insets at 63x. (Scale bars $=30 \mu \mathrm{m})$. 
A

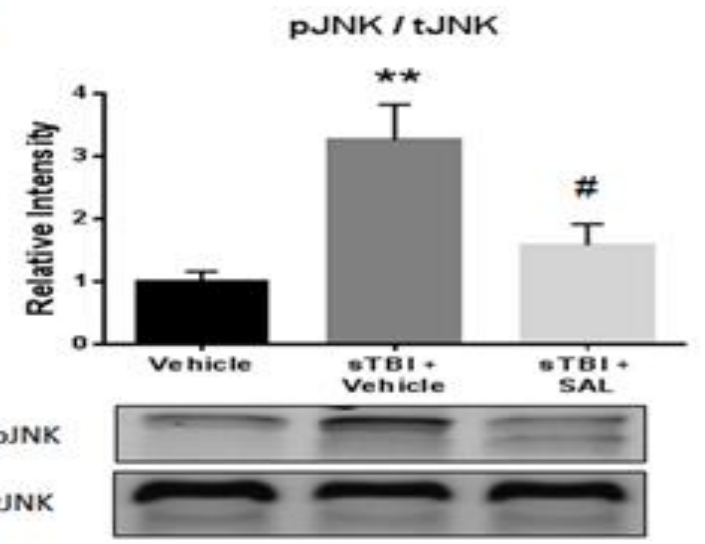

C

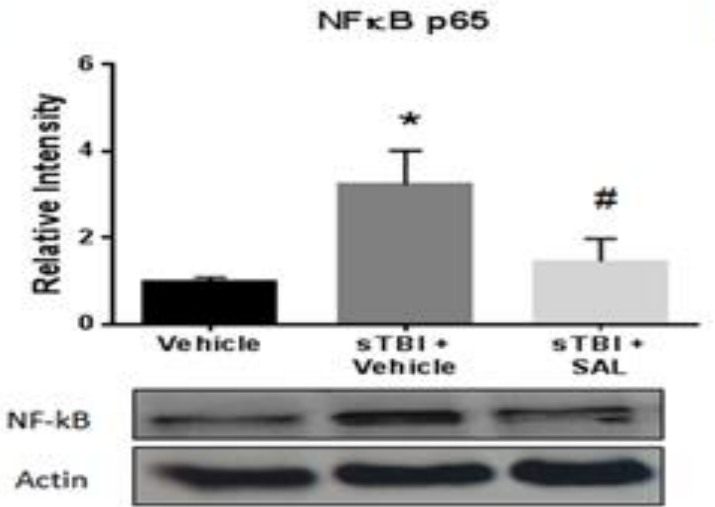

$\mathbf{E}$

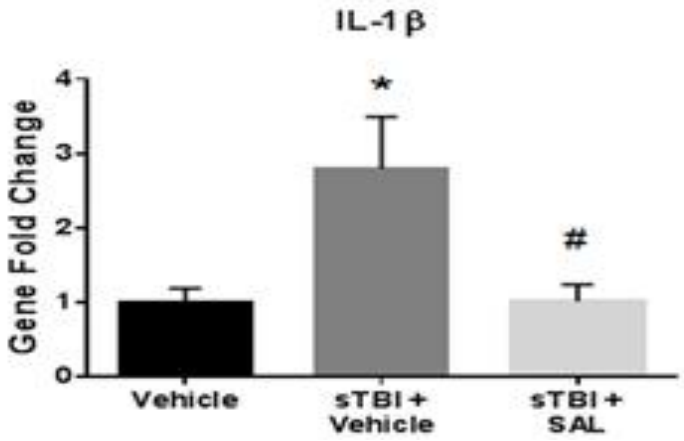

B

BiP

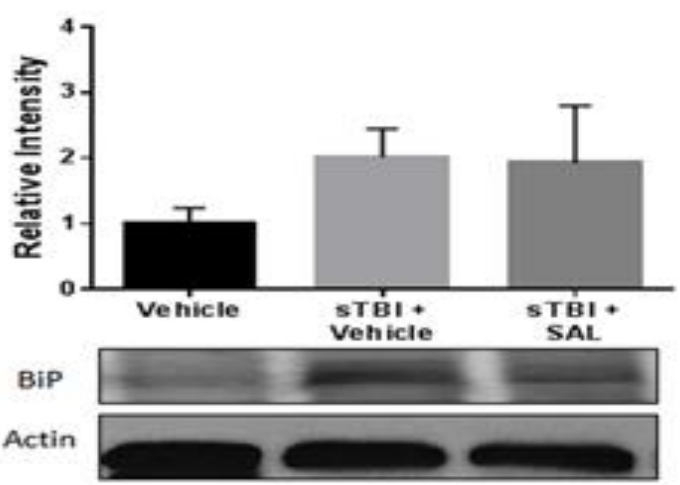

D

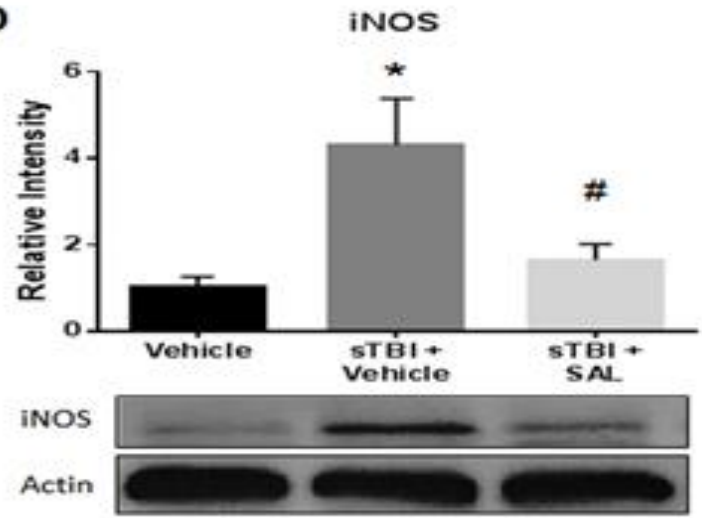

$\mathbf{F}$

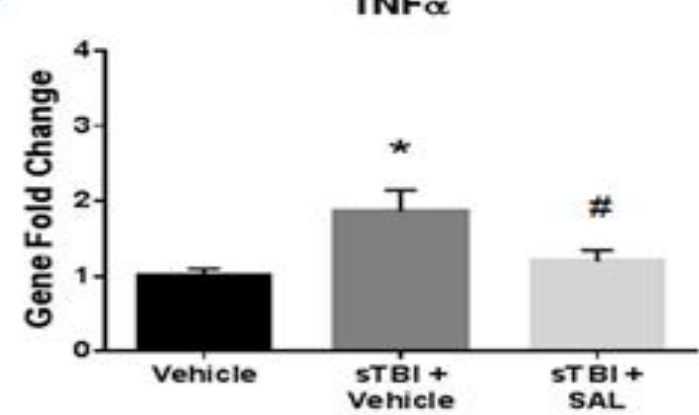

Figure 5.4. SAL mitigated markers of neuroinflammation after single blast

(A) We measured a significant increase in JNK phosphorylation at 24 hours post-sTBI $(* * \mathrm{P}<$ 0.01 vs Vehicle); SAL significantly mitigated JNK phosphorylation (\#P $<0.05$ vs sTBI + Vehicle). (B) No significant differences were observed in BiP expression at 24 hours post-sTBI $(\mathrm{P}>0.05)$. (C) We measured a significant increase in NFkB p65 expression at 24 hours postsTBI $(* \mathrm{P}<0.05$ vs Vehicle); SAL significantly mitigated NFKB p65 expression $(\# \mathrm{P}<0.05$ vs sTBI + Vehicle). (D) We revealed a significant increase in iNOS protein expression at 24 hours post-sTBI ( $* \mathrm{P}<0.05$ vs vehicle); SAL significantly mitigated iNOS expression (\#P $<0.05$ vs sTBI + Vehicle). (E) We measured a significant increase in IL-1 $\beta$ mRNA abundance at 24 hours post-sTBI ( $* \mathrm{P}<0.05$ vs Vehicle); SAL significantly mitigated IL- $1 \beta$ abundance (\#P $<0.05$ vs sTBI + Vehicle). (F) We measured a significant increase in TNF $\alpha$ mRNA abundance at 24 hours post-sTBI $(* \mathrm{P}<0.05$ vs Vehicle); SAL significantly mitigated TNF $\alpha$ abundance $(\# \mathrm{P}<0.05)$. One-way ANOVA, Newman-Keul's post hoc. Mean \pm S.E.M. $n=4$. 


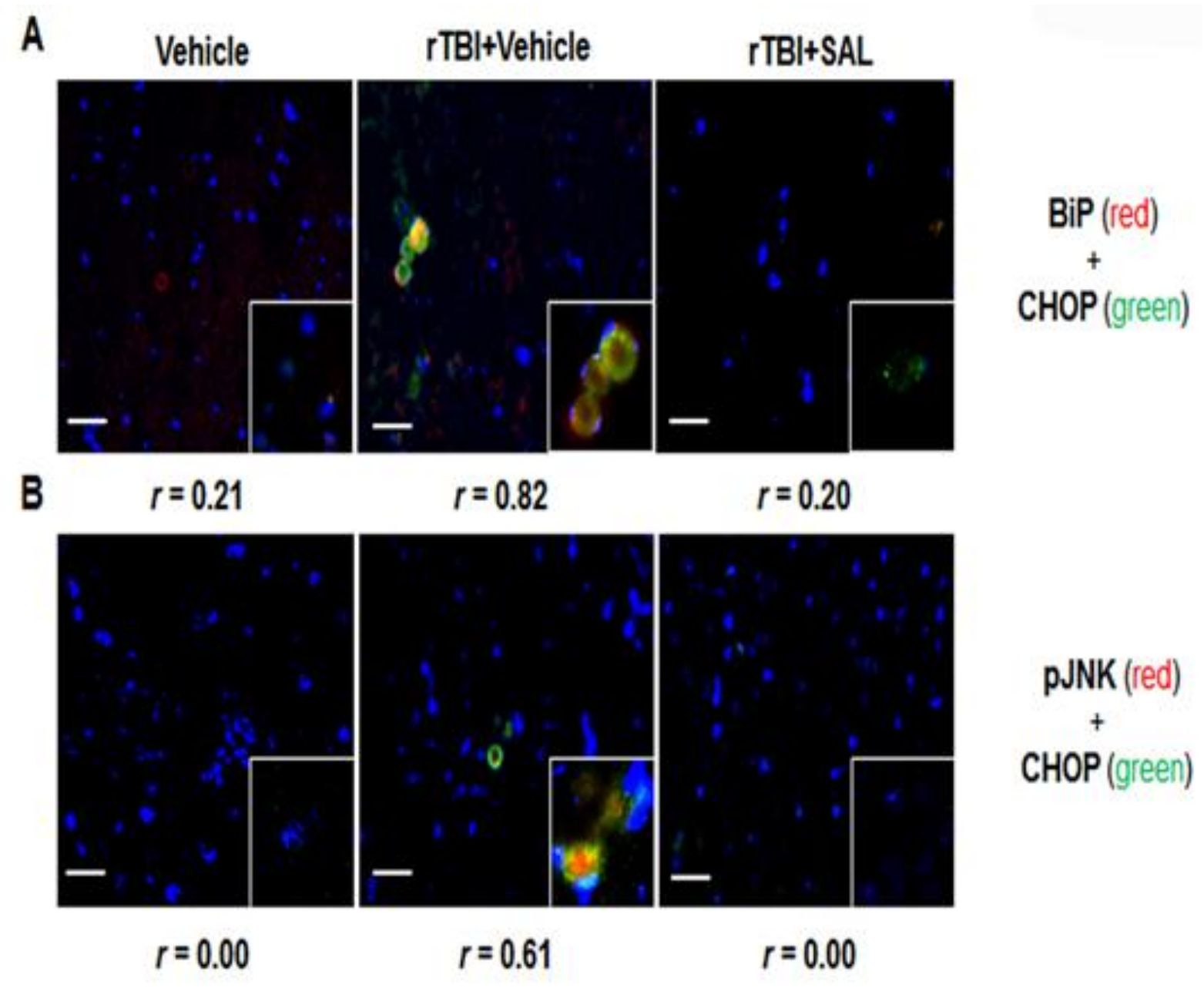

Figure 5.5. BiP and pJNK colocalized with CHOP after repetitive blast

Columns are separated by experimental group: Vehicle, rTBI + Vehicle, and rTBI + SAL (left to right). All panels display nuclear counterstain DAPI (blue). (A) BiP (red) merged with CHOP (green). (B) pJNK (red) merged with CHOP (green). Colocalization determined by levels of yellow in each image (Overlap coefficient; $r$ values). All images are from the frontal cortex region. Images are displayed at 20x; Insets displayed at 63x. (Scale bars $=30 \mu \mathrm{m})$. 


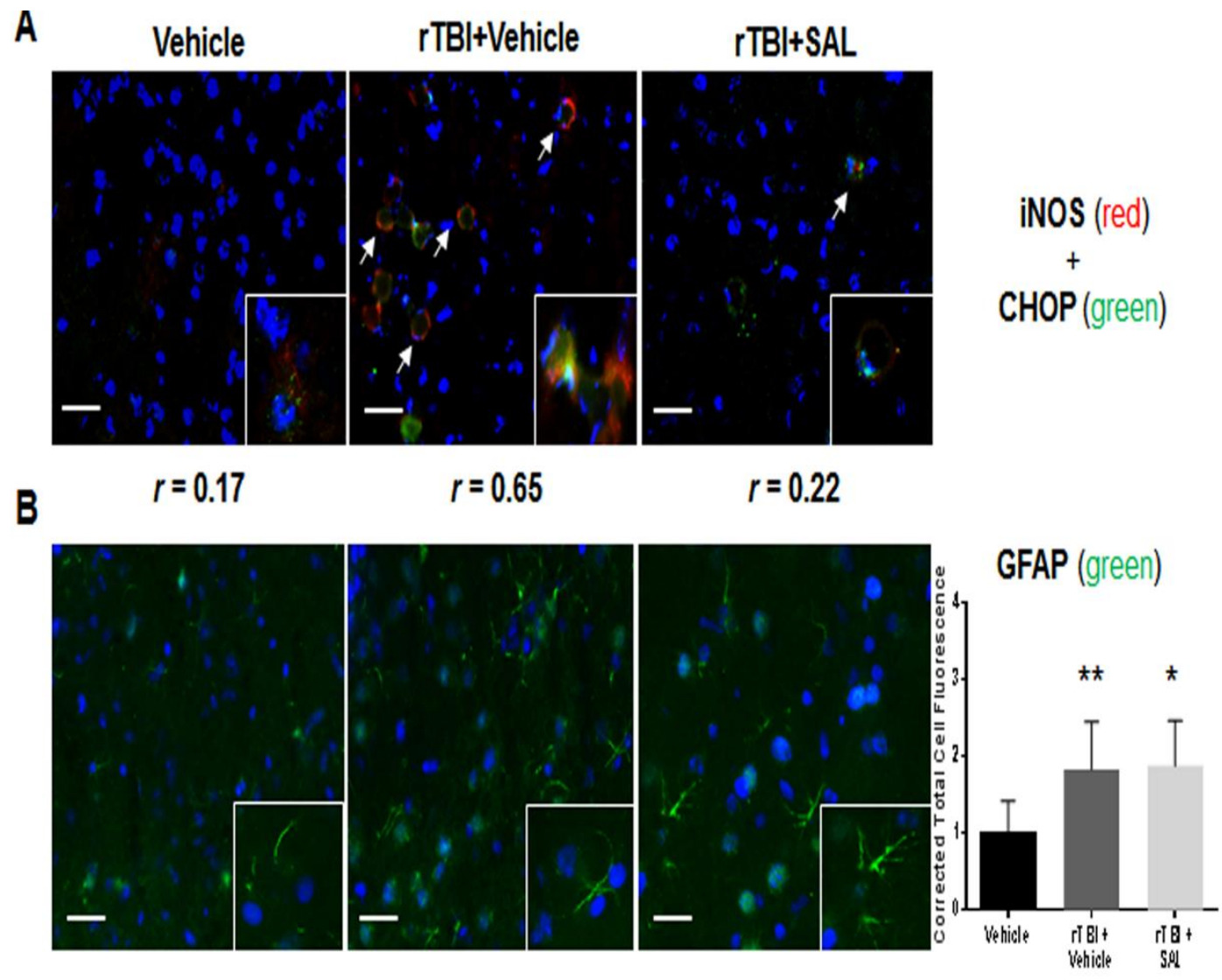

Figure 5.6. SAL reduced iNOS fluorescence, but not GFAP, after repetitive blast

Columns are separated by experimental group: Vehicle, rTBI + Vehicle, and rTBI + SAL (left to right). All panels display nuclear counterstain DAPI (blue). (A) iNOS (red) merged with CHOP (green). Colocalization was determined by levels of yellow in each image (Overlap coefficient; $r$ values). Arrows demarcate iNOS fluorescence. (B) We observed a significant increase in GFAP (green) fluorescence at 72 hours post-rTBI $(* * \mathrm{P}<0.01$ vs Vehicle); we also observed a significant increase in GFAP post-rTBI with SAL administration ( $* \mathrm{P}<0.05$ vs Vehicle). Oneway ANOVA, Newman-Keul's post hoc. Mean \pm S.E.M. $n=4$. All images are from the frontal cortex region. Images are displayed at 20x; Insets displayed at 63x. (Scale bars $=30 \mu \mathrm{m})$. 


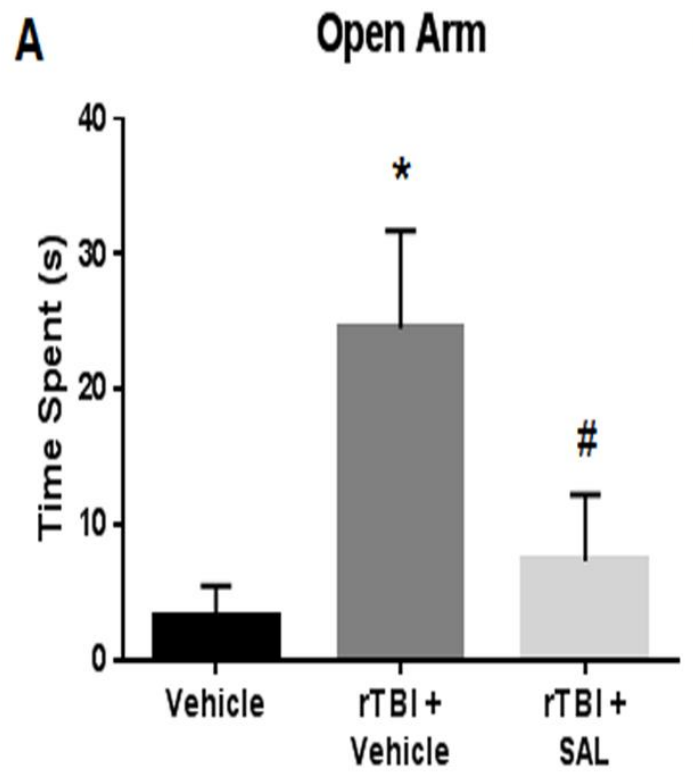

B Total Movement

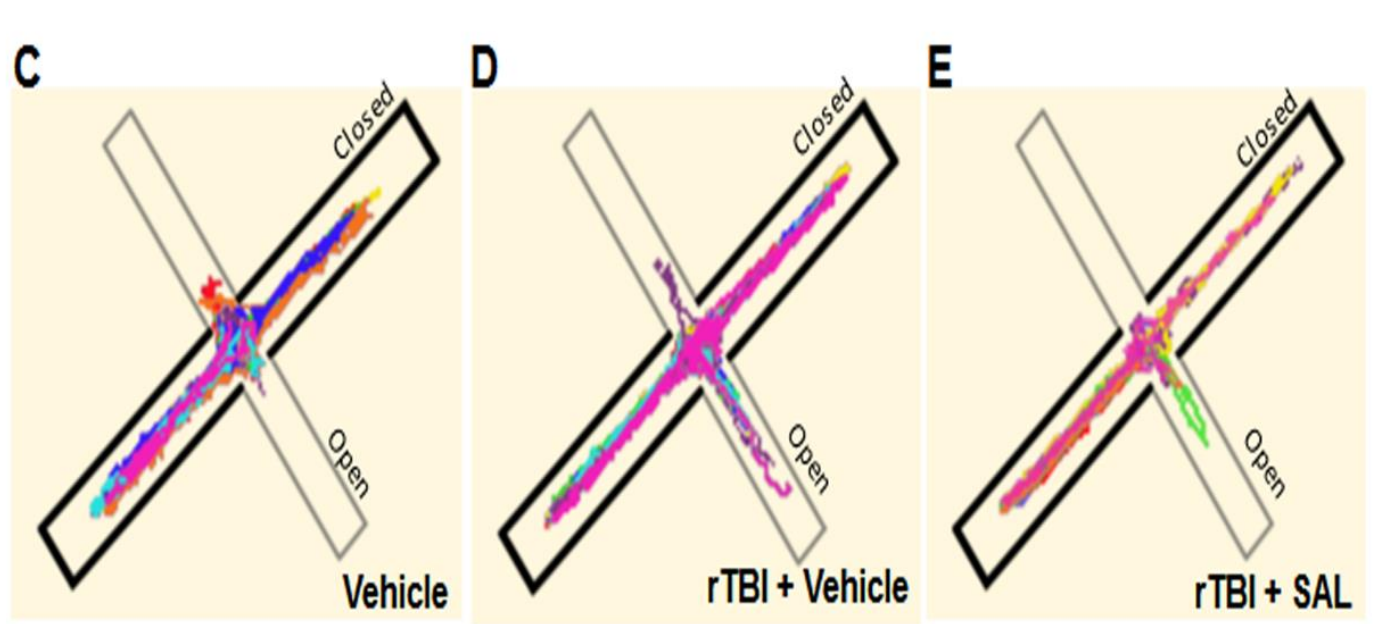

Figure 5.7. SAL ameliorated risk-taking behavior after repetitive blast

(A) A significant increase was observed in the time that the rats spent in the open arms of the EPM at 72 hours post-rTBI $(* \mathrm{P}<0.05$ vs Vehicle); SAL significantly reduced the time spent (\#P $<0.05$ vs rTBI + vehicle). (B) No significant differences were observed in the total distance moved among rats in each group $(\mathrm{P}>0.05)$ One-way ANOVA, Newman-Keul's post hoc. Mean \pm S.E.M. $n=9 .(C, D, E)$ Track plots from Anymaze ${ }^{\mathrm{TM}}$ were overlaid for each subject to provide a clear visual representation of the rats' behavior during the EPM trials. 


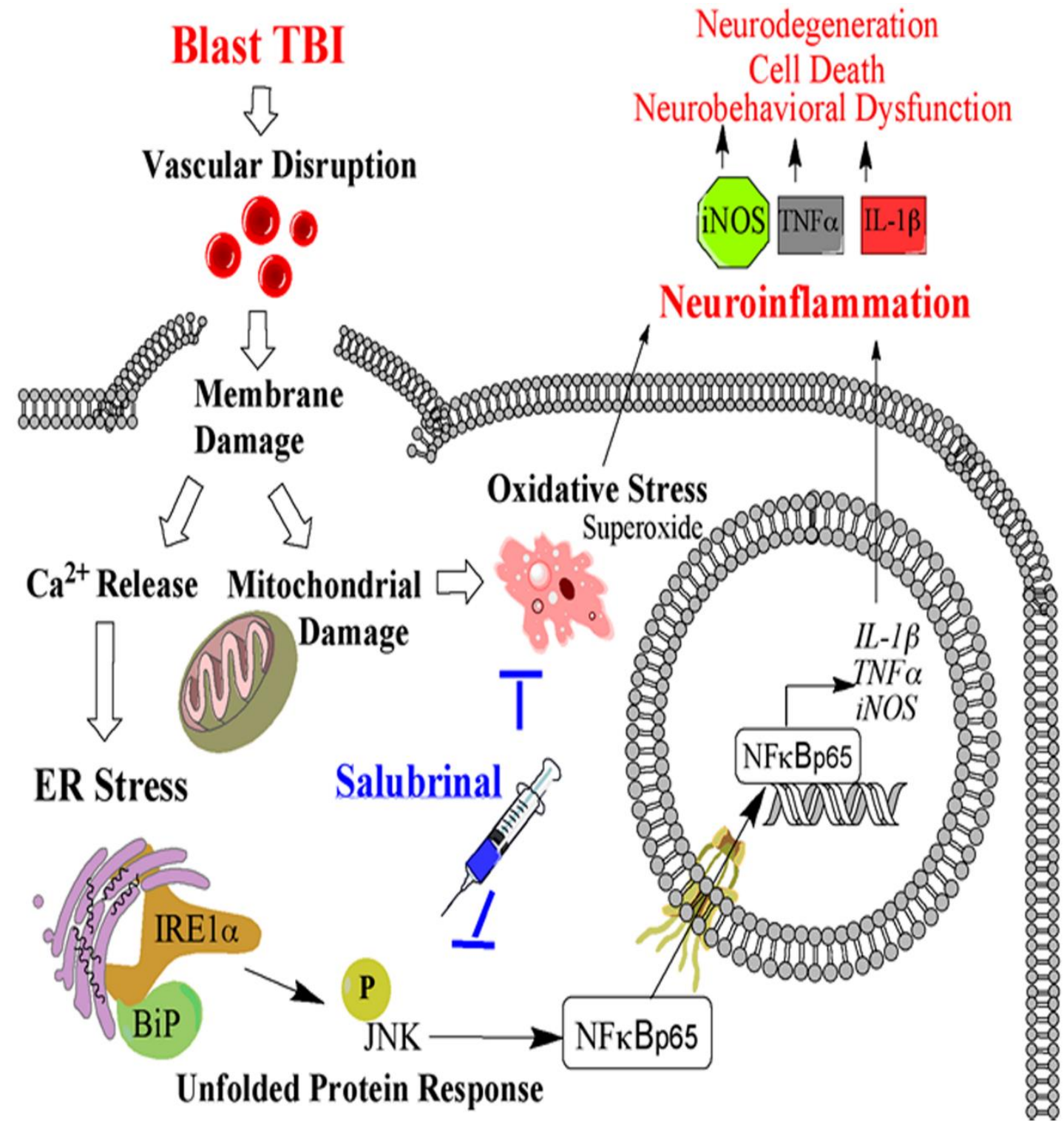

Figure 5.8. Blast injury cascade with SAL mechanism of action

Schematic of blast injury cascade and salubrinal mechanism of action. TBI $=$ Traumatic Brain Injury; ER = Endoplasmic Reticulum; IRE1 $\alpha=$ Inositol requiring enzyme 1 alpha; BiP $=$ Binding immunoglobulin protein; $\mathrm{NF \kappa B}=$ Nuclear factor kappa beta; JNK = Janus n-terminal kinase; iNOS $=$ inducible Nitric oxide synthase; TNF $\alpha=$ Tumor necrosis factor alpha; IL-1 $\beta=$ Interleukin 1 beta. 


\section{Chapter Six}

\section{The quest to model chronic traumatic encephalopathy: a multiple model and injury paradigm experience}

This work is published in Front Neurol. 2015 Oct 20;6:222.

doi: 10.3389/fneur.2015.00222. eCollection 2015. PMID: 26539159

Turner RC*, Lucke-Wold BP*, Logsdon AF*, Robson MJ, Dashnaw ML, Huang JH, Smith KE, Huber JD, Rosen CL, Petraglia AL. 


\section{ABSTRACT}

Chronic neurodegeneration following a history of neurotrauma is frequently associated with neuropsychiatric and cognitive symptoms. In order to enhance understanding about the underlying pathophysiology linking neurotrauma to neurodegeneration, a multi-model preclinical approach must be established to account for the different injury paradigms and pathophysiologic mechanisms. We investigated the development of tau pathology and behavioral changes using a multi-model and multi-institutional approach, comparing the preclinical results to tauopathy patterns seen in post-mortem human samples from athletes diagnosed with chronic traumatic encephalopathy (CTE). We utilized a scaled and validated blast-induced traumatic brain injury model in rats and a modified pneumatic closed-head impact model in mice. Tau hyperphosphorylation was evaluated by western blot and immunohistochemistry. Elevated plus maze and Morris water maze were employed to measure impulsive-like behavior and cognitive deficits respectively. Animals exposed to single blast ( 50 PSI reflected peak overpressure) exhibited elevated AT8 immunoreactivity in the contralateral hippocampus at 1 month compared to controls. Animals exposed to repeat blast (6 blasts over 2 weeks) had increased AT8 and AT270 in the contralateral hippocampus at 1 month post-injury compared to controls. In the modified controlled closed-head impact mouse model, no significant difference in AT8 was seen at 7 days, however a significant elevation was detected at 1 month following injury in the ipsilateral hippocampus compared to control. Elevated plus maze data revealed that rats exposed to single blast and repeat blast spent more time in seconds exploring the open arms compared to controls. Morris water maze testing revealed a significant difference between groups in acquisition times on days 22-27. During the probe trial, single blast and repeat blast rats spent less time in seconds exploring where the platform had been located compared to controls. This study provides a multi-model example of replicating tau and behavioral changes in animals and provides a foundation for future investigation of CTE disease pathophysiology and therapeutic development. 


\section{INTRODUCTION}

Current limitations in understanding CTE pathophysiology are unlikely to be addressed in a clinical population in the near future due to the challenges associated with establishing longterm, prospective cohort studies in such a population. Preclinical rodent models serve to fill this gap in knowledge for various disorders and could allow for further investigation of the molecular mechanisms responsible for CTE, as well as testing the potential of diagnostic and therapeutic approaches under development. Few preclinical models of CTE have been proposed that sufficiently demonstrate both the requisite tauopathy and behavioral changes attributed to CTE (DeFord et al., 2002; DeRoss et al., 2002; Uryu et al., 2002; Creeley et al., 2004; Longhi et al., 2005; Friess et al., 2009; Blaylock and Maroon, 2011; Shitaka et al., 2011; Fujita et al., 2012b; Goldstein et al., 2012; Mouzon et al., 2012; Turner et al., 2012a; Genovese et al., 2013; Hawkins et al., 2013; Klemenhagen et al., 2013; Mannix et al., 2013; Aungst et al., 2014; Bailes et al., 2014; Donovan et al., 2014; Lucke-Wold et al., 2014a; Mannix et al., 2014; Mouzon et al., 2014; Petraglia et al., 2014b; Petraglia et al., 2014a; Brody et al., 2015).

The purpose of this paper is to present two preclinical models that successfully reproduce some neuropathological and behavioral changes consistent with CTE-like phenotypes, and discuss future directions for CTE animal modeling. Brody and colleagues (Brody et al., 2015) present several unanswered questions that we expand upon in our accompanying review such as the role of 1) inter-injury interval, 2) number of impacts, 3) impact severity, 4) age at time of impacts, 5) mechanism of impact, 6) genetics, 7) gender, and 8) effect of environment on the likelihood and/or progression of CTE development. We recently reported that endoplasmic reticulum stress might be a contributing factor linking acute neurotrauma to behavioral deficits (Logsdon et al., 2014). 
The quest for elucidating CTE pathophysiology development is ongoing with the goal of targeting specific injurious cascades a key priority in order to prevent the emergence of clinical symptoms.

In this work we present novel models of neurotrauma-induced neurodegeneration in both mice and rats following exposure to single or repetitive brain injury, respectively. These models replicate both the tauopathy and some of the behavioral changes implicated in CTE in the clinical population. We believe the blast model is particularly relevant clinically due to utilization of a scaled, short-duration blast exposure. This is a striking contrast to long-duration blasts utilized in numerous studies that may more closely approximate an atomic blast than a blast from an improvised explosive device (Bass et al., 2012; Panzer et al., 2012; Turner et al., 2013b; Panzer et al., 2014). Park and colleagues show that by having the animal outside of the tube, the wave is directed towards the skull causing neurologic injury without extensive lung injury (Park et al., 2011). Extended duration waves generated from long blast tubes are based on lung based scaling. When the animals are placed within the tube, the impulse exposure to the skull might not be representative of human exposure (Cho et al., 2013). The modified closed-head injury in unanesthetized animals described previously by Petraglia and colleagues offers the benefit of injuring awake animals, more closely replicating the clinical picture seen in athletes than most other TBI models (Petraglia et al., 2014b; Petraglia et al., 2014a). Further studies are required to address injury paradigms that do or do not contribute to CTE development but utilization of these models appears promising in not only modeling CTE but also identifying therapeutic targets based upon other recently published work by our groups (Logsdon et al., 2014; Lucke-Wold et al., 2014c).

\section{METHODS}




\section{Animals and Human Samples}

All experiments involving animals were approved by either the Institutional Animal Care and Use Committee of West Virginia University or that of the University of Rochester and were performed based upon principles of the Guide for the Care and Use of Laboratory Animals. Fifty six (56) young-adult male rats (300-350 grams) were acquired from Hilltop Laboratories (Scottdale, PA). All blast procedures were performed at West Virginia University. Twelve (12) young-adult male C57BL/6J mice were acquired from Jackson Laboratories (Bar Harbor, ME) and used for modified controlled cortical impact at the University of Rochester. All animals were allowed to acclimate upon arrival for 1 week prior to any experimentation. At all times animals were provided food and water ad libitum and maintained on a 12 hour light-dark cycle. Human samples were from deceased professional athletes that were previously diagnosed with chronic traumatic encephalopathy (Omalu et al., 2010b). The tissue was collected from the entorhinal cortices.

\section{Experimental Groups}

Fifty six $(n=56)$ rats were divided into three primary experimental groups for behavior anesthetized controls $(n=24)$, a single blast injury $(n=16)$, and repeat blast injuries $(n=16)$. Each of these groups was sacrifed at 1 month following the final blast or sham-injury and after undergoing functional assessment. Elevated-plus maze was done at 7 days post-blast $(\mathrm{n}=24 ; 8$ controls, 8 single blast, and 8 repeat blast) and the Morris water maze started at 21 days post-blast ( $\mathrm{n}=32 ; 16$ controls, 8 single blast, and 8 repeat blast). Following behavioral analysis, rats from the EPM group were divided into two separate groups with one utilized for immunohistochemistry $(\mathrm{n}=10 ; 4$ controls, 3 single blast, and 3 repeat blast $)$ and the other for western blotting $(\mathrm{n}=14 ; 4$ controls, 5 single blast, and 5 repeat blast). Twelve (12) mice were divided into three primary 
experimental groups - anesthetized controls $(n=4)$, single injury with sacrifice at one week $(n=$ 4), and single injury with sacrifice at one month $(n=4)$. All mice were utilized for western blotting techniques at time of sacrifice. An experimental schematic can be seen in Figure 1.

\section{Traumatic Brain Injury}

Briefly, all blast injuries administered to rats as part of this work were of moderate intensity ( 50 PSI peak reflected overpressure) as determined previously and completed under isofluranebased anesthesia (Turner et al., 2013b; Logsdon et al., 2014; Lucke-Wold et al., 2014c). Blast exposure occurred on the right side of the animal and was only administered to the head and neck region. The rat was outside of the blast tube and a rigid barrier protected the remainder of the animal. Blast waves were of a short-duration $(\sim 2 \mathrm{~ms})$ to ensure clinical relevance based on elucidated scaling parameters (Figure 2A). The scaling parameters are based on impulse dynamic measurements, which are more representative of human blast than closed models based on duration (Needham et al., 2015).

Mice were utilized in a manner previously described (Petraglia et al., 2014b; Petraglia et al., 2014a). Briefly, mice were placed, unanesthetized, into a rodent restraint bag/cone and immobilized on top of a foam bed of known spring constant. A helmet was secured to the head using an elastic band, allowing for administration of a diffuse impact through force distribution. Impacts were delivered using a modified controlled cortical impact device that was adjusted to include an altered tip of vulcanized rubber (Figure 2B).

\section{Western Blot}

Animals were anesthetized and sacrificed by rapid decapitation. Brains were extracted and immediately placed in a lysate buffer with protease and phosphatase inhibitors as described elsewhere (Logsdon et al., 2014). Brains were dissected and tissue flash frozen and stored at -80 
${ }^{\circ} \mathrm{C}$ prior to blotting. Hippocampal protein samples were dissolved in $0.5 \mathrm{~mL}$ of $1 \%$ sodium dodecyl sulfate (SDS) prior to sonication and protein assay. Pre-cast 10\% 12-well gels (Life Technologies, Carlsbad, CA) were loaded with $30 \mu \mathrm{g}$ of protein per well and run with $2 \mathrm{X}$ Lammeli buffer. Wet transfer was performed using nitrocellulose membranes (Bio-Rad, Contra Costa, CA) at 60 volts for 2.5 hours. Primary antibodies against AT8 (Pierce; Rockford, IL), AT270 (Pierce; Rockford, IL), CP13 (kindly supplied by Dr. Peter Davies), and PHF-1 (kindly supplied by Dr. Peter Davies) were utilized and detected utilizing the corresponding secondary. Membranes were imaged using a LI-COR fluorescent scanner (LI-COR; Lincoln, NE) and images converted to gray scale. Analysis was performed using background subtraction (Odyssey Processing Software, LICOR) and values normalized to $\beta$-actin levels, resulting in a normalized intensity value.

\section{Immunohistochemistry}

For immunohistochemistry preparation, animals were anesthetized using isoflurane and transcardially perfused with ice-cold physiologic saline followed by $10 \%$ formalin for a total of 10 minutes. Brains were extracted and placed in fresh $10 \%$ formalin for a minimum of 24 hours prior to blocking and subsequent paraffin embedding. Sections were prepared in $6 \mu \mathrm{m}$ thickness using a Leica RM2235 microtome (Leica Microsystems, Wetzlar, Germany). Staining was performed using standard protocols used within the field and previously by our laboratory, using the antibodies described above as well as thioflavin for detecting neurofibrillary tangles (McKee et al., 2010; Goldstein et al., 2012; Stern et al., 2013).

\section{Behavioral Assessments}

Learning and memory was assessed using the Morris water maze. Spatial acquisition trials began at Day 21 after the final blast exposure (in both single and repeat injury paradigms). The pool utilized was $\sim 180 \mathrm{~cm}$ in diameter and filled with water at ambient temperatures $\left(18-21{ }^{\circ} \mathrm{C}\right)$. 
A platform $(10 \mathrm{~cm} \times 10 \mathrm{~cm})$ was submerged $2.5 \mathrm{~cm}$ below the surface of the water. A series of objects was placed in the environment around the pool to provide visual cues for the animal during trials. The training paradigm (spatial acquisition) consisted of 6 days with a total of 4 trials occurring each day per animal. Animals were placed into the maze from four different locations each day (4 trials) with a total of 2 minutes (maximum) allowed per trial. Upon finding the platform, animals were allowed 15 seconds for acquisition to occur. If unsuccessful in finding the platform, animals were placed on the platform at the conclusion of 2 minutes by the investigator. On the probe trial day (platform removed), animals were placed in the maze at a novel location and allowed to explore the maze for 1 minute. Data was acquired using AnyMaze ${ }^{\mathrm{TM}}$ video tracking software (Stoelting Co., Wood Dale, IL) throughout all studies and allows for acquisition of latency, distance, and speed data to be analyzed across maze regions/quadrants.

Impulsivity was determined, at 7 days after the final blast, as previously described using an elevated-plus maze and measuring exploratory behavior (Mosienko et al., 2012; Johnson et al., 2013a; Logsdon et al., 2014). The apparatus was placed at a height of $60 \mathrm{~cm}$ from the floor and consisted of two open and two closed arms, with open arms opposing one another and intersecting perpendicularly with the opposed closed arms. Each arm was $50 \mathrm{~cm}$ long by $10 \mathrm{~cm}$ wide. Open arms were surrounded by clear plastic edging $\sim 1.5 \mathrm{~cm}$ high. Closed arms were encased with black walls $30 \mathrm{~cm}$ tall, creating a 3-sided and comforting enclosure for the rodent. At the start of each 5-minute trial, animals were placed in the middle of the intersecting arms facing an open arm prior to release. Animals were allowed to explore the apparatus for the duration of the trial. AnyMaze ${ }^{\mathrm{TM}}$ software was utilized to record the animals' position, distance traveled, and entry pattern into various arms throughout the trial. An increased percentage of time spent in the open arms was 
considered a sign of impulsive behavior (Mosienko et al., 2012; Johnson et al., 2013a; Logsdon et al., 2014).

Statistical Analysis

An observer blinded to experimental condition performed all data acquisition. One-way Analysis of Variance (ANOVA) was used for statistical analysis of all tests except spatial acquisition trials of the Morris water maze and immunohistochemical comparisons in which a twoway, repeated measures ANOVA and students t-test were utilized, respectively. Bonferroni posthoc comparison was used to determine differences between experimental groups on two-way ANOVA with repeated measures for spatial acquisition. For all other comparison's, a Tukey's post-hoc was utilized. Analysis was completed using GraphPad Prism 5.0 (GraphPad Software, Inc.; La Jolla, CA). A $p<0.05$ was considered statistically significant for all data analyzed.

\section{RESULTS}

\section{Neurotrauma induces tau hyperphosphorylation in both blast and closed-head injury models}

To elucidate the effect of neurotrauma on the development of CTE-like neuropathology, animals were exposed to sham-injury, single-injury (either blast or modified closed-head), or repeat-injury (blast). Animals were first assessed for tau phosphorylation, using AT8 and AT270 antibodies, believed to be the initial precursor of neurofibrillary tangle development. AT8 forms a single band in rats and a double band in mice (Hawkins et al., 2013). Conversely, AT270 forms a double band in rats, but only a single band in mice (Green et al., 2007). The number of bands is indicative of calpain dependent phosphorylation, which is regulated uniquely between rats and mice (Medeiros et al., 2012). Increased phosphorylation of tau was observed after blast exposure and modified closed-head injury but the location of the increase was model-dependent. 
Specifically, tau phosphorylation was observed in the contralateral hemisphere in rats exposed to repeat blast injury but only in the ipsilateral hemisphere, and only with the AT8 antibody, in mice receiving a single modified closed-head injury.

In rats exposed to blast, no significant difference in tau phosphorylation was observed in the ipsilateral hippocampus after blast injury for AT8 (Fig. 3A) or AT270 (Fig. 3E). A significant difference was observed in AT8 $\left(\mathrm{F}_{2,11}=16.64, p<0.001\right)$ and AT270 $\left(\mathrm{F}_{2,9}=4.37, p<0.05\right)$ levels in the contralateral hippocampus of rats exposed to blast injury. At one month following a single blast, a significant increase in tau phosphorylation recognized by AT8 increase was measured compared to control $(\mathrm{q}=3.96, p<0.05)$ (Fig. 3B). A significant increase in phosphorylation detected by AT8 was also observed after repeat blast $(\mathrm{q}=8.12, p<0.001)$ (Fig. 3B).

In the modified closed-head injury model utilized on the mice, a significant difference was observed in tau phosphorylation AT8 $\left(\mathrm{F}_{2,9}=4.93, p<0.05\right)$ in the ipsilateral hippocampus. At one-month following injury, AT8 expression was elevated in comparison to sham-injury $(\mathrm{q}=4.34$, $p<0.05$ ) (Fig. 3C). No significant difference was observed at 7-days post-injury on the ipsilateral side or between any groups or time points on the contralateral side (Fig. 3D).

At one-month following repetitive blast exposure, a significant increase phosphorylation recognized by the AT270 antibody was measured in the contralateral hippocampus of rats compared to anesthetized control animals $(\mathrm{q}=4.03, p<0.05)$ (Fig. 3F). No significant differences were observed in tau phosphorylation recognized by AT270 in either ipsilateral (Fig. 3G) or contralateral (Fig. 3H) hippocampus of mice receiving modified closed-head injury.

\section{Hyperphosphorylation of tau following neurotrauma occurs in perivascular brain regions}

Clinical case series documenting patients with CTE have demonstrated the deposition of neurofibrillary tangles (NFTs), an end product of tau hyperphosphorylation, in perivascular 
regions, which is a distinct difference from other tauopathies such as Alzheimer's disease (AD). Possible mechanisms leading to NFTs in perivascular regions include the disruption of the bloodbrain barrier (BBB), leading to punctate microhemorrhages, red blood cell breakdown, oxidative stress, and finally, persistent neuroinflammation. We observed a significant difference in tau hyperphosphorylation within perivascular regions of the contralateral hippocampus in rats exposed to repeat blast in comparison to control when identified with both AT8 $(\mathrm{t}=4.46, p<0.001)$ and AT270 $(\mathrm{t}=11.47, p<0.001)$ at 1-month post-injury (Fig. 4). These findings were consistent with prior reports of CTE in humans and from our collection of post-mortem human samples with documented CTE.

Neurotrauma is associated with conformational changes in tau that are recognized precursors of neurofibrillary tangle formation

Following hyperphosphorylation, tau is purported to undergo conformational changes associated with subsequent insolubility and deposition/precipitation in the form of neurofibrillary tangles. Following neurotrauma in rats and mice, we found an elevation in markers of conformational change of the tau molecule based on immunoblotting with PHF-1 and CP13. After blast exposure, markers of tau conformational change were observed in the contralateral hemisphere of rats, while after the impact procedure in mice, the markers were found ipsilaterally. No significant differences in markers of tau conformation change were observed in the ipsilateral hippocampus after blast injury for neurofibrillary precursor PHF-1 (Fig. 5A) or CP13 (Fig. 5E).

A significant difference was observed in PHF-1 $\left(\mathrm{F}_{(2,11)}=7.92, p<0.01\right)$ and $\mathrm{CP} 13\left(\mathrm{~F}_{(2,9)}=\right.$ 6.03, $p<0.05)$ levels in the contralateral hippocampus of rats exposed to blast injury. At one month following a single blast, a significant increase in PHF expression was measured compared to control $(q=4.76, p<0.05)($ Fig. $5 B)$. 
A significant difference was observed in PHF-1 $\left(\mathrm{F}_{(2,9)}=7.18, p<0.05\right)$ levels in the ipsilateral hippocampus of mice exposed to injury. At one month following modified impact, a significant increase in PHF-1 expression was measured compared to control $(\mathrm{q}=5.17, p<$ 0.05)(Fig. 5C). No significant differences were observed in PHF-1 expression in the contralateral hippocampus of mice exposed to cortical impact (Fig. 5D).

At one month after repetitive blast exposure, a significant increase in CP13 expression was measured in the contralateral hippocampus of rats compared to control $(\mathrm{q}=4.73, p<0.05)$ (Fig. 5F). A significant difference was observed in $\mathrm{CP} 13\left(\mathrm{~F}_{(2,9)}=21.70, p<0.001\right)$ levels in the ipsilateral hippocampus of mice exposed to injury. At one month following impact, a significant increase in CP13 expression was measured compared to control $(q=9.28, p<0.001)($ Fig. $5 \mathrm{G})$. No significant differences were observed in CP13 expression in the contralateral hippocampus of mice exposed to cortical impact (Fig. 5H).

Similar findings were seen using immunohistochemistry when comparing repeat-injured animals to sham-injured animals at 1 month post-injury. Specifically, a significant difference was seen on PHF-1 staining $(\mathrm{t}=6.06, p<0.001)$ and CP-13 staining $(\mathrm{t}=3.88, p<0.01)($ Fig. 6$)$. Again, the distribution of the staining was notable for being perivascular in nature, a finding shared across the clinical specimens diagnosed with CTE as seen in Fig. 7. We show that PHF, AT8, and a thioflavin stained neurofibrillary tangle are increased perivascular in human CTE specimens. CP13 is increased in a perivascular distribution following repeat blast in a rat.

\section{Neurotrauma produces cognitive impairments}

Cognitive deficits have long been associated with the neuropathological diagnosis of CTE in the clinical population, particularly amongst the population diagnosed with CTE at a later age in life. Similarly, cognitive impairments in both learning and memory have been associated with 
neurotrauma but have not been presented in the context of a corresponding tauopathy in both a single and repeat blast injury paradigm. In cohorts of animals subjected to either sham-, single-, or repetitive-injury, spatial acquisition (learning) was assessed 3 weeks after the injury in singleinjury animals and the final injury in repeat-injury paradigms (Days 21-27 post-injury). Blast injury exposure was associated with worsened performance in the Morris water maze as evident by latency to find the platform in seconds when analyzed using a two-way repeated measures ANOVA. Post-hoc tests revealed significant differences between anesthetized controls and singleinjury animals during spatial acquisition on Days 22-24, and Day 26 (Day 21: $\mathrm{t}=1.38, p>0.05$; Day 22: $\mathrm{t}=3.70, p<0.01$; Day 23: $\mathrm{t}=4.12, \mathrm{p}<0.001$; Day 24: $\mathrm{t}=4.38, p<0.001$; Day 25: $\mathrm{t}=$ 2.17, $p>0.05$; Day 26: $\mathrm{t}=3.20, p<0.01$ ). Similarly, post-hoc tests demonstrated a significant impairment in acquisition between anesthetized controls and repetitively injured animals on Days 22-26 (Day 21: $\mathrm{t}=0.66, p>0.05$; Day 22: $\mathrm{t}=5.01, p<0.001$; Day 23: $\mathrm{t}=4.16, p<0.001$; Day 24: $\mathrm{t}=4.72, p<0.001$; Day 25: $\mathrm{t}=3.69, p<0.01$; Day 26: $\mathrm{t}=3.76, p<0.01)$. Notably, no differences were seen between single- and repeat-injured animals during the course of acquisition trials, despite the notable difference in tau pathology at this time presented earlier (Fig. 8). The differences in latency to platform between blast-injured animals and anesthetized control animals is apparent as well based on visualization of the track plots recorded during data acquisition (Fig. 9). Clearly different swimming patterns emerge with blast-injured animals exhibiting what appears to be more thigmotaxis (circling around the outer edge of the pool) than anesthetized control animals that reached statistical significance between controls and repeat-injured animals on Day $23(\mathrm{q}=3.58, p<0.05)$, although this data point did not reach significance on Days 21 and 26 based on automated measurements generated using AnyMaze ${ }^{\mathrm{TM}}$ (Fig. 8). 
Memory, as measured during the probe test (time spent in area surrounding the nowremoved platform) conducted on Day 27, again demonstrated deficits in animals subjected to blastinduced neurotrauma when analyzed using a one-way ANOVA $\left(\mathrm{F}_{2,29}=20.01, p<0.0001\right)$. Posthoc tests showed significant differences between sham and single injury animals $(\mathrm{t}=6.44, p<$ $0.05)$ as well as between sham and repetitively injured animals $(\mathrm{t}=8.00, p<0.05)$ but no difference between single injury and repeat injury paradigms (Fig. 8). These findings were confirmed visually using track plots generated during data acquisition (Fig. 9). Anesthetized control animals exhibit a greater preponderance of pool crossings and swimming behavior within the region near the platform in contrast to blast-injured animals (Fig. 9).

\section{Impulsivity is increased following neurotrauma}

Clinically, impulsive behavior has been described extensively in those with a history of repetitive neurotrauma and the context of CTE. To make an analogous comparison between our animal model of blast-induced traumatic brain injury and clinically reported symptoms, the elevated-plus maze was utilized to measure impulsive-like behaviors based upon the percentage of time spent in the open-arms of the maze. Significant differences between groups were observed when analyzed using a one-way ANOVA $\left(\mathrm{F}_{2,32}=5.03, p<0.05\right)$. Post-hoc tests revealed a significant difference between sham and single injury animals $(\mathrm{q}=3.53, p<0.05)$ as well as between sham and repetitively injured animals $(\mathrm{q}=4.21, p<0.05)$. No difference was seen between single and repeat injury paradigms with regards to percentage of time spent in the open arms of the apparatus (Fig. 10). While not statistically significant, a trend towards a greater distance traveled in the open arms was observed with increasing levels of neurotrauma, evidence consistent with the percentage of time spent in the open arms (Fig. 10). Conversely, a trend was also present regarding distance traveled in the closed arms with animals subjected to neurotrauma 
traveling less distance (Fig. 10). Post-hoc tests revealed no statistical significance when comparing sham to single injury groups but significance was reached when comparing sham to repetitivelyinjured animals $\left(\mathrm{F}_{2,33}=3.06 ; \mathrm{q}=3.47, p<0.05\right)$.

\section{DISCUSSION}

While the clinical literature has been inundated with reports of CTE in athletes and soldiers alike, few experimental tools exist for investigating disease pathophysiology, establishing diagnostic criteria, and discovering preventative or therapeutic agents. For these reasons, preclinical models of CTE are highly desirable. In this work we demonstrate two preclinical models capable of generating some biochemical and behavioral hallmarks of CTE, namely tauopathy and impulsive-like behavior. Furthermore, this work illustrates the utility of two distinct models and injury paradigms, namely blast exposure versus a more traditional modified closedhead injury, and repeat versus single injury paradigms respectively. Likewise, the fact that this work was completed in two different species and builds upon prior work by both West Virginia University and the University of Rochester laboratories validates the use of both mice and rats in the preclinical modeling of neurotrauma related neurodegeneration (Logsdon et al., 2014; Petraglia et al., 2014b; Petraglia et al., 2014a). The modified closed-head injury represents a helmeted design with controlled placement of the impact likely accounting for ipsilateral deficit. The tauopathy development period following modified controlled impact was not previously elucidated therefore warranting the 7-day time point. Petraglia and colleagues show that astrocyte activation and cell death occurs at 7 days post modified controlled impact (Petraglia et al., 2014a). We previously reported that tauopathy following blast in rats does not develop until weeks after injury and is on the contralateral side due to coup/contra-coup injuries (Lucke-Wold et al., 2015c). 
The potential utility of these, as well as other preclinical models of neurotrauma related neurodegeneration, is highly promising for investigation of disease pathophysiology, particularly as related to biochemical endpoints associated with CTE. To fully develop a CTE model, transgenic rodents will be needed that include amyloid, tau, and TDP43 pathology. The quest for elucidating the underlying mechanisms behind CTE development is ongoing. In previous work, we have shown blast causes substantial blood brain barrier disruption (Lucke-Wold et al., 2014c), endoplasmic reticulum stress activation (Logsdon et al., 2014), and oxidative stress (Lucke-Wold et al., 2015a). We show in this work the induction of hyperphosphorylated tau, conformational changes in tau, and more advanced precursors of NFT formation with the usage of CP13 and PHF1 antibodies. AT8 binds to serine 199 and 202 as well as threonine 205 . AT270 binds to threonine 181, PHF binds to serine 396 and 404, and CP13 binds to serine 202. Serine 396 and 202 are only exposed after tau undergoes conformational change (Lucke-Wold et al., 2014b). These changes are the ultimate result of tau hyperphosphorylation and protein misfolding/aggregation, and are likely related to dysregulation of the tau kinase/phosphatase system. Peclinical modeling of neurotrauma related neurodegeneration will allow for the elucidation of how and when these kinases and phosphatases become dysregulated, and will allow for increased understanding of the disease process. Additionally, these studies will provide targets for direct or indirect therapeutic development. Similarly, preclinical models may prove instrumental in identification of diagnostic and prognostic tests for establishing a diagnosis of CTE and tracking disease progression. There is a clinical need for a rapid, cheap, and reliable diagnostic test to predict severity of CTE. Currently neuropathological examination remains the gold standard for diagnosis with some studies purporting the use of PET-based imaging for diagnosis (Small et al., 2013; Gandy and 
DeKosky, 2014; Mitsis et al., 2014). It is possible that development and validation of these techniques, and others such as diffusion tensor imaging and magnetic resonance spectroscopy may be accelerated through the application to preclinical models. The current limitation is that advanced imaging is expensive, cumbersome, and requires expertise from subspecialty radiologists.

Despite this work illustrating what we believe represents a clear step forward in the study of neurotrauma related neurodegeneration, it is clear that further study is warranted. Pre-clinical models must be utilized to more fully characterize behavioral, biochemical, imaging, and electrophysiological/functional changes associated with the development of CTE. Future studies will likely address the development of behavioral/functional deficits in relation to biochemical changes temporally and assess the chronicity of changes based on the number, severity, and inter-injury interval of neurotrauma-related events. The development of better transgenic models is critical as the field moves forward. Performing studies such as these will allow for the questions raised in our accompanying review be addressed. Specifically, what is the role of the 1) inter-injury interval, 2) number of impacts, 3) impact severity, 4) age at time of impacts, 5) mechanism of impact, 6) genetics, 7) gender, and 8) effect of environment on the likelihood and/or progression of CTE development. 

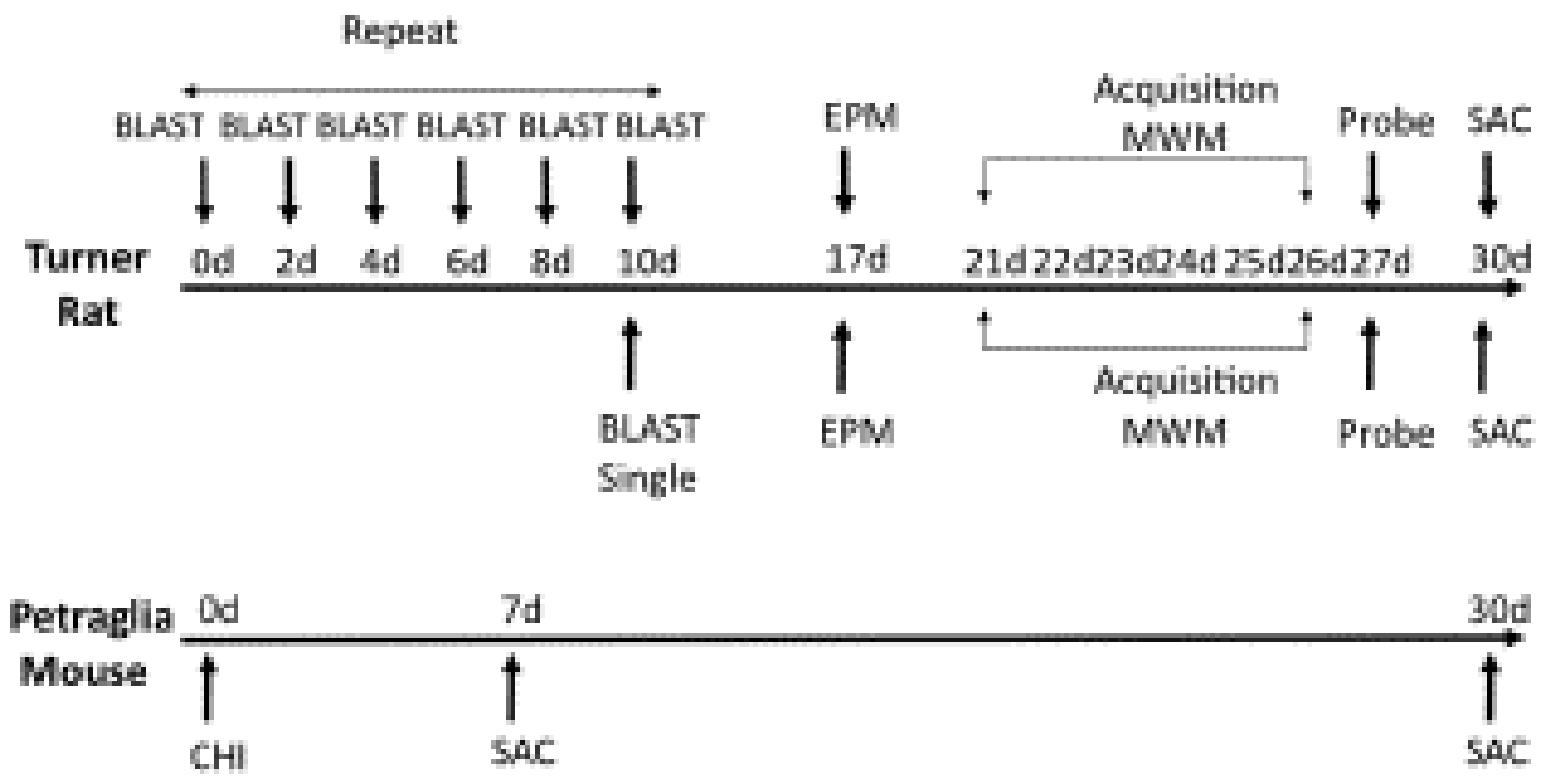

Figure 6.1. Schematic showing experimental design and behavioral experiments. Time of sacrifice for biochemical experiments is also shown. 


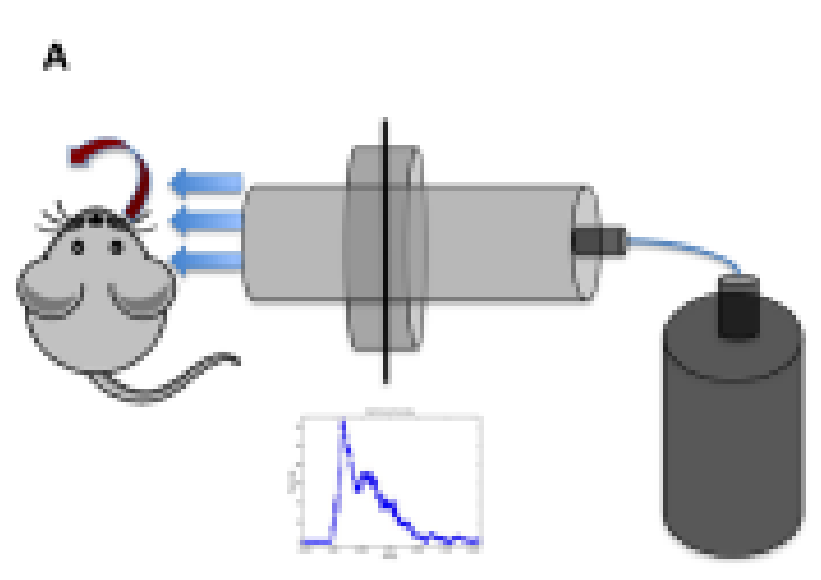

Blast Wave
B

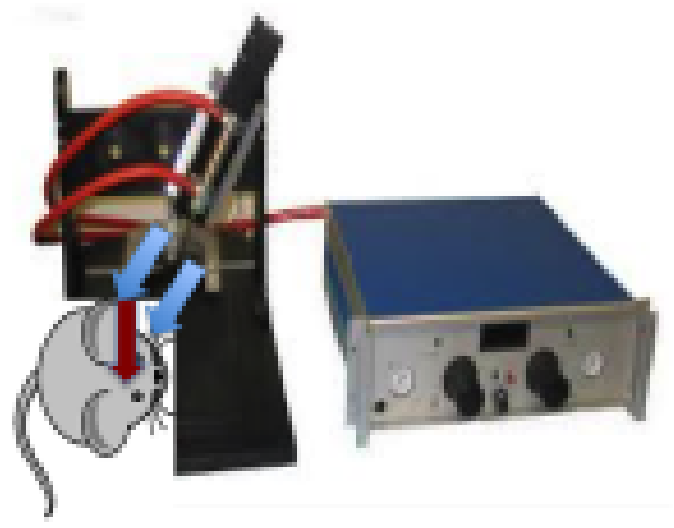

Closed Head lmpact

Acceleration-Deceleration Forces

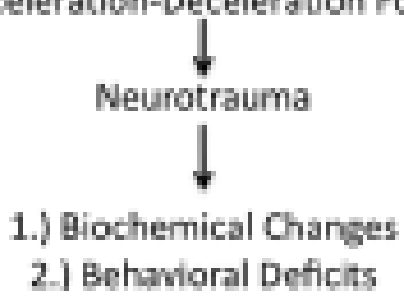

Figure 6.2. Schematic representation of the two injury models utilized within this work. (A) Experimental setup utilized at West Virginia University for the study of blast-induced neurotrauma. The shock tube consists of a high-pressure driver section (nitrogen gas filled) and a low-pressure driven section (ambient air filled). When the membrane dividing the chambers ruptures, the blast wave is formed and released, encountering the rat from the right side of the cranium. (B) Depiction of the model utilized at the University of Rochester in un-anesthetized mice. An electromagnetic impactor used in CCI was modified with a rubber tip and a specially designed helmet was placed on the mouse as previously described. 


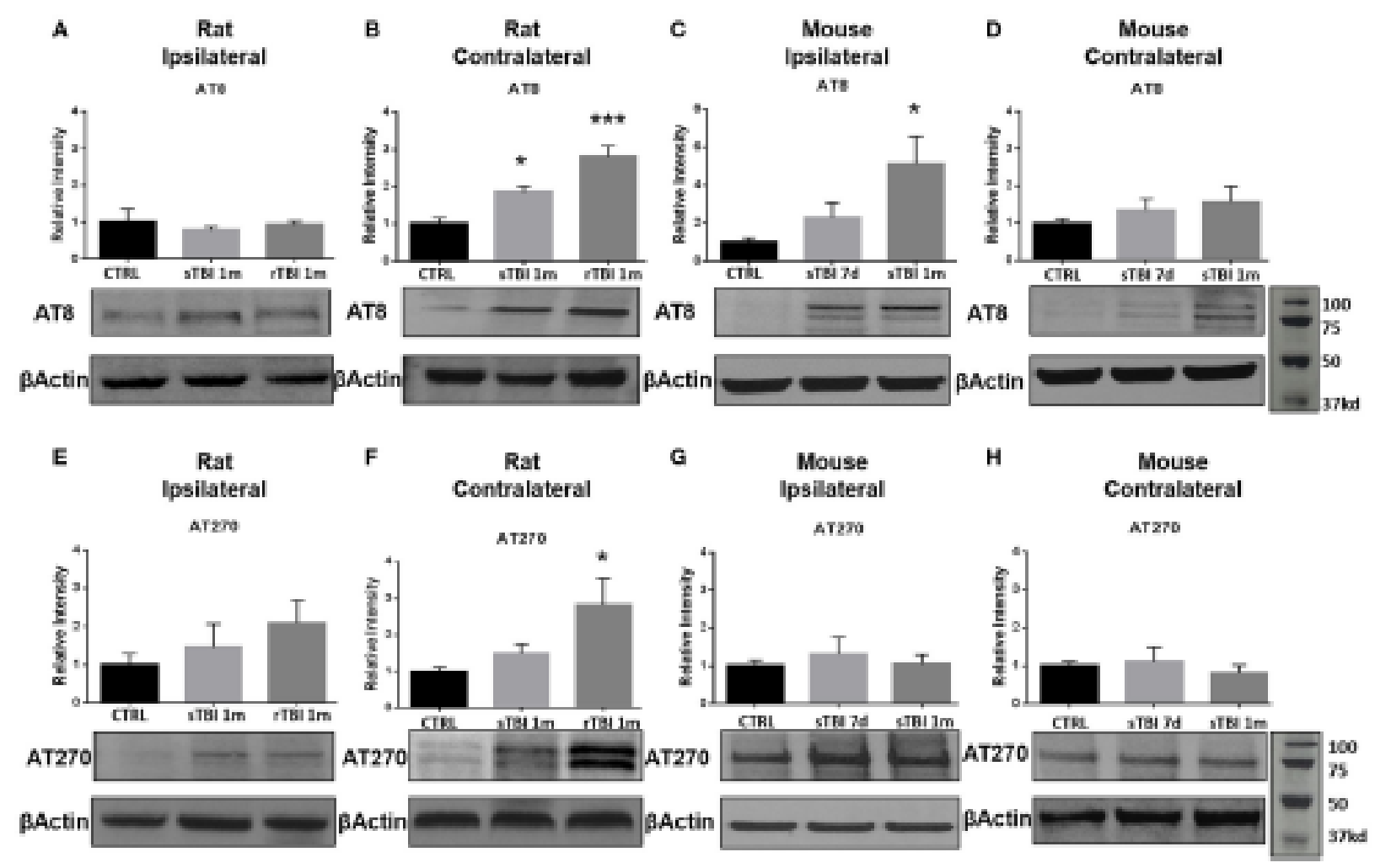

Figure 6.3. Blast and closed-head injury models both induced tau hyperphosphorylation. (A) Immunoblots show no significant difference in tau phosphorylation at serine sites 199/202 and threonine site 205 (AT8) at one month after single and repeat blast exposures in the ipsilateral rat hippocampus. (B) A significant increase in AT8 expression was measured after single $*^{*} p<0.05$ vs. CTRL) and repeat blast exposure $(* * * p<0.001$ vs. CTRL) in the contralateral rat hippocampus. (C) AT8 expression was significantly increased at one month after closed-head injury in the ipsilateral mouse hippocampus ( ${ }^{*} p<0.05$ vs. CTRL). (D) No significant differences were observed in AT8 expression in the contralateral mouse hippocampus. (E) Immunoblots show no significant difference in tau phosphorylation at threonine site 181 (AT270) at one month after single and repeat blast exposure in the ipsilateral rat hippocampus. (F) A significant increase in AT270 expression was measured at one month after repeat blast exposure ( ${ }^{*} p<0.05$ vs. CTRL) in the contralateral rat hippocampus. (G) No significant differences were observed in AT270 expression in the ipsilateral mouse hippocampus, $(\mathbf{H})$ or the contralateral mouse hippocampus after closedhead injury. One-way ANOVA Tukey's post-hoc analysis (values represent mean \pm s.e.m.; normalized to $\beta$-actin) $(n=3-5)$. 

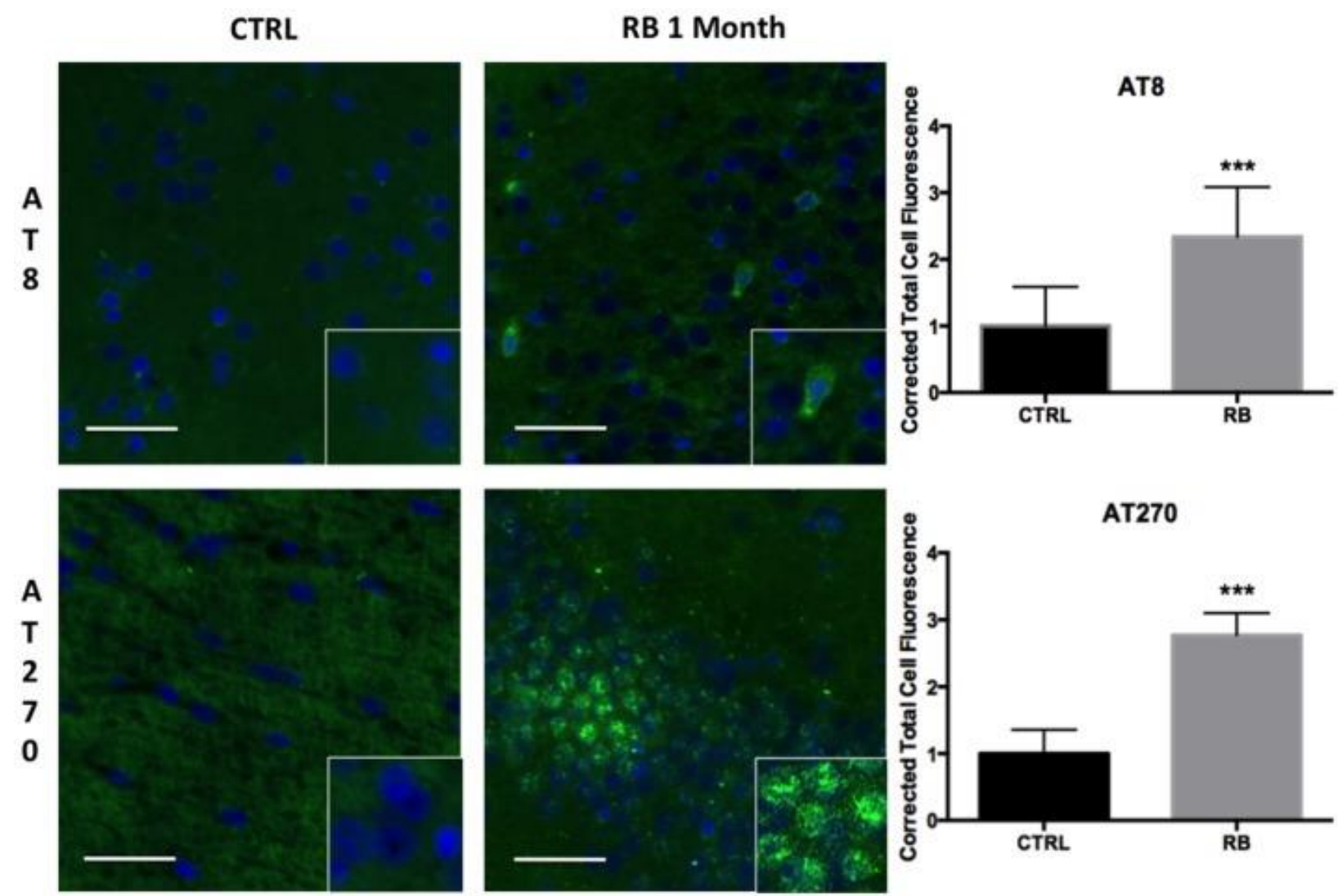

Figure 6.4. Tau hyperphosphorylation is seen in microfoci throughout the contralateral superficial cortex. AT8 was significantly increased at 1 month post repetitive blast $(\mathrm{t}=4.455, p$ $<0.001)$. AT270 was also significantly increased following repetitive blast $(\mathrm{t}=11.47, p<0.001)$. AT270 was increased in a circular distribution. Tau hyperphosphorylation is an indicator of progressive pathology. Values were calculated using a student's t-test comparing the mean difference between groups. 


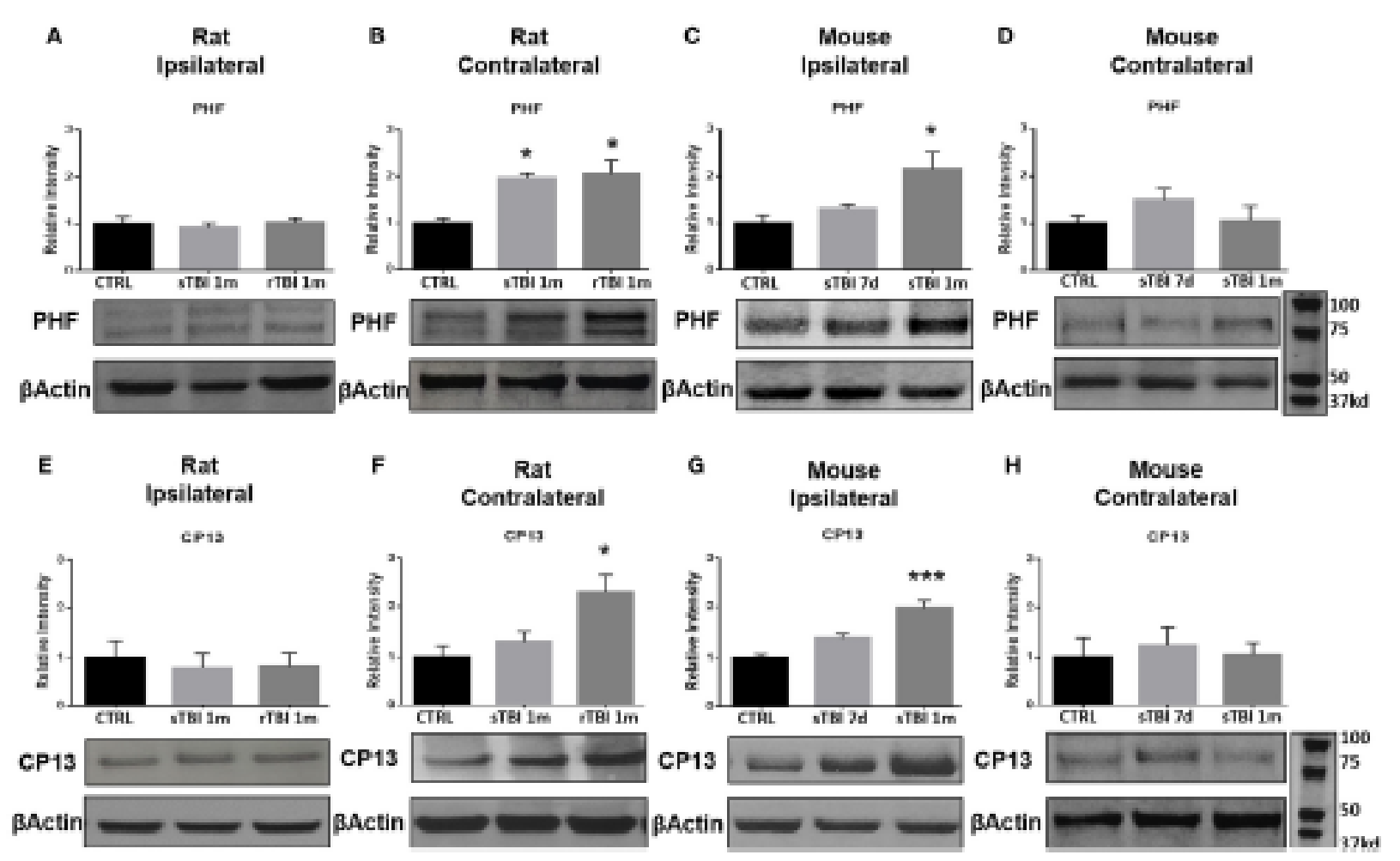

Figure 6.5. Blast and closed-head injury models both induce conformational changes in tau proteins. (A) Immunoblots show no significant difference in markers of tau conformational change at serine site 396/404 (PHF) at one month after single and repeat blast exposure in the ipsilateral rat hippocampus. (B) A significant increase in PHF expression was measured after single ( ${ }^{*} p<0.05$ vs. CTRL) and repeat blast exposure $(* p<0.05$ vs. CTRL) in the contralateral rat hippocampus. (C) PHF expression was significantly increased at one month after closed-head injury in the ipsilateral mouse hippocampus. (D) No significant differences were observed in PHF expression in the contralateral mouse hippocampus. (E) Immunoblots show no significant difference in markers of tau conformational change at serine site 202 (CP13) at one month after single and repeat blast exposure in the ipsilateral rat hippocampus. (F) A significant increase in CP13 expression was measured at one month after repeat blast exposure ( ${ }^{*} p<0.05$ vs. CTRL) in the contralateral rat hippocampus. (G) A significant increase in CP13 expression was measured at one month after closed-head injury in the ipsilateral mouse hippocampus $(* * * p<0.001$ vs. CTRL). (H) No significant differences were observed in CP13 expression in the contralateral mouse hippocampus after closed-head injury. One-way ANOVA Tukey's post-hoc analysis (values represent mean \pm s.e.m.; normalized to $\beta$-actin) $(n=3-5)$. 

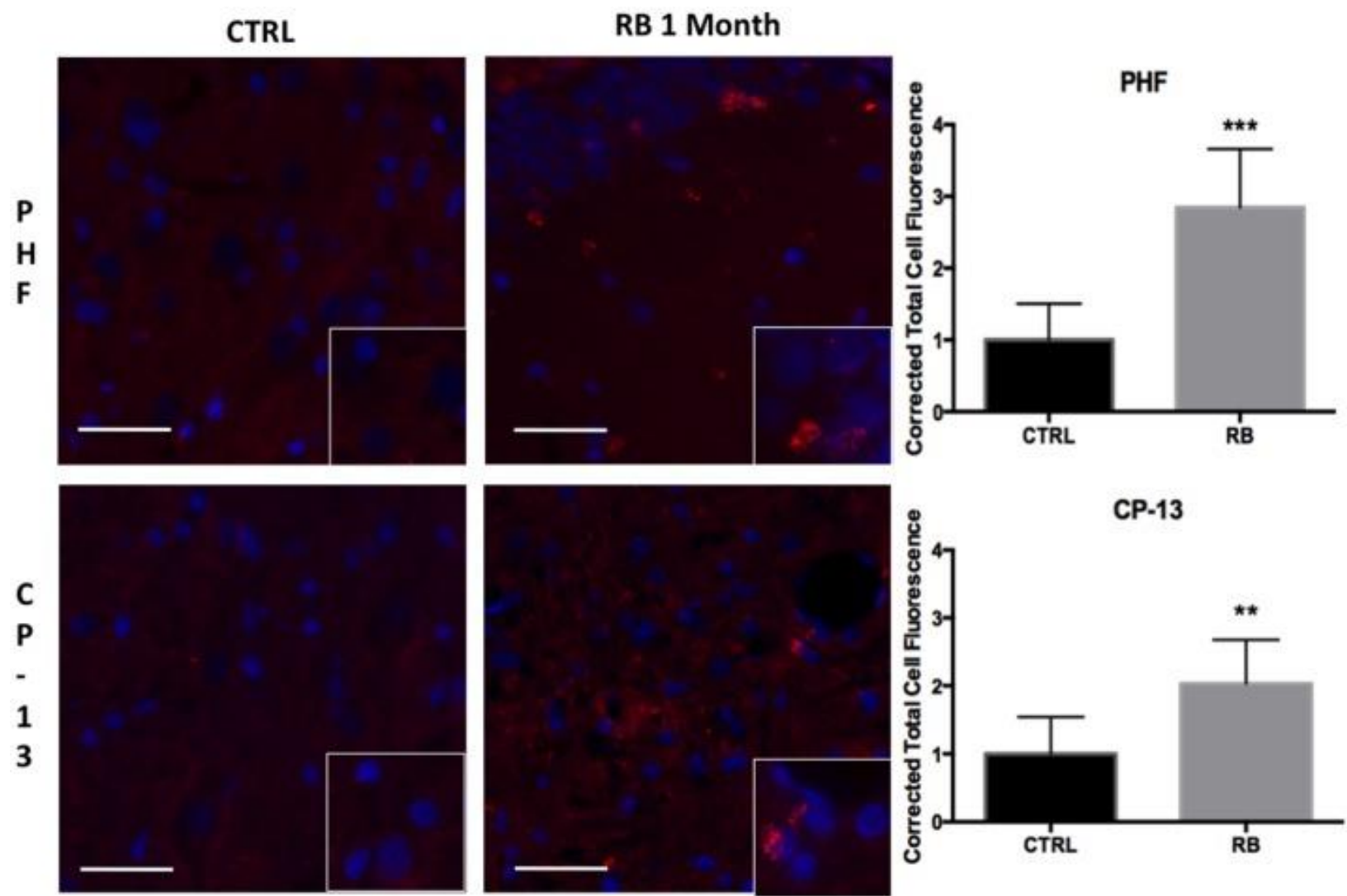

Figure 6.6. Tau conformational markers were increased throughout the contralateral superficial cortex. PHF was significantly increased at 1 -month post repetitive blast $(\mathrm{t}=6.055, p$ $<0.001)$. CP-13 staining was also significantly increased following repetitive blast $(\mathrm{t}=3.883, p<$ 0.01). CP-13 was increased in a perivascular distribution. Tau conformational change is required for the formation of neurofibrillary tangles. Values were calculated using a student's t-test comparing the mean difference between groups. 


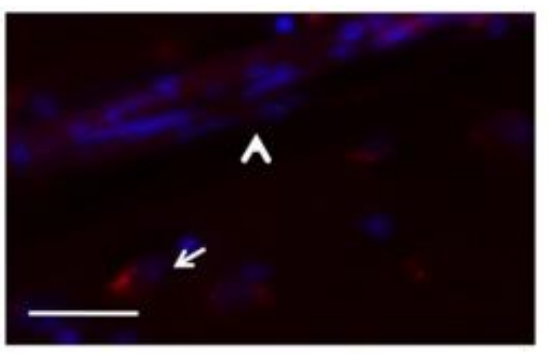

Human PHF

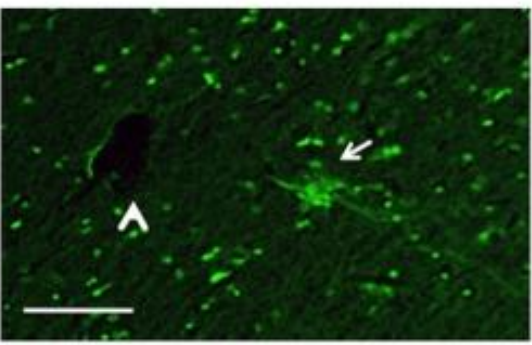

Human NFT
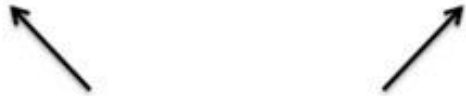

Perivascular Tau 1
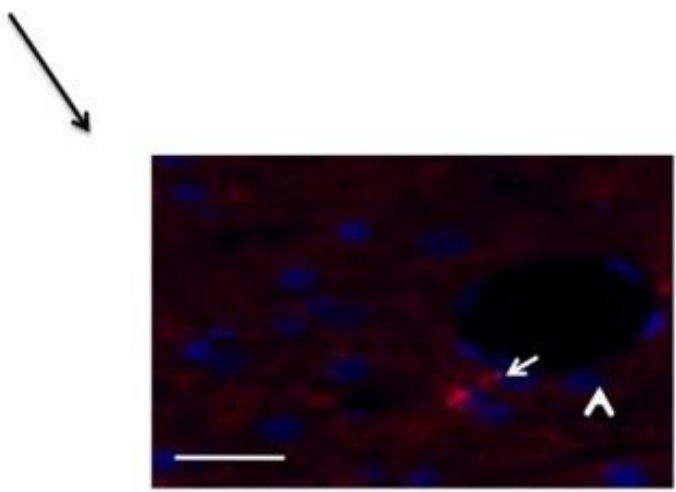

Rat CP-13

Figure 6.7. Tauopathy is seen in a perivascular distribution. PHF and AT8 were increased adjacent to longitudinal microvessels in human patients diagnosed with chronic traumatic encephalopathy. A neurofibrillary tangle stained with thioflavin was seen adjacent to a cut vessel lumen in the brain of a retired professional football player. Similarly, CP-13 was increased next to a cut vessel lumen in the contralateral cortex 1 month following repeat blast in the rat. Arrows indicate tauopathy while arrow heads point out vessels. 

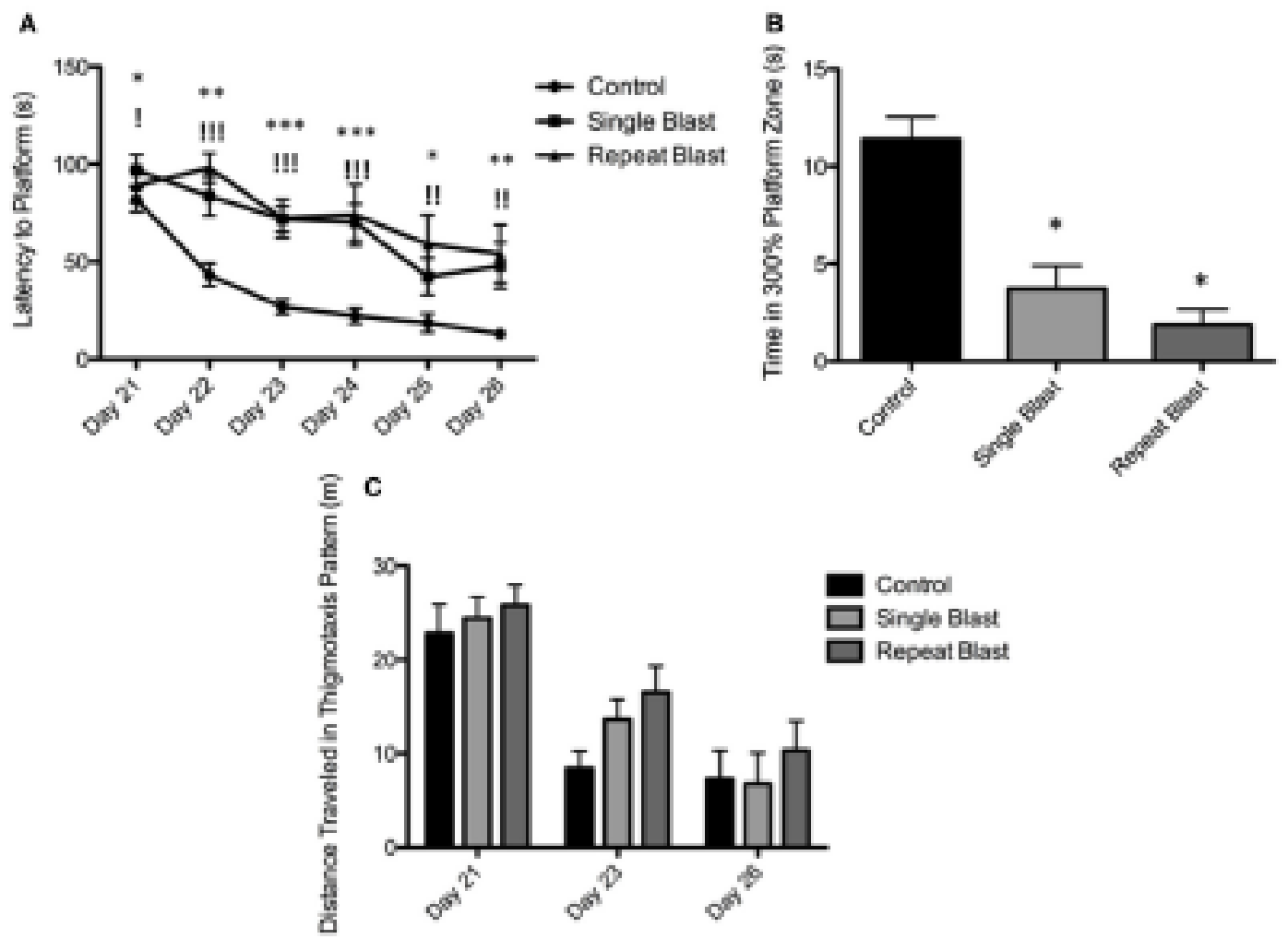

Figure 6.8. Blast-induced brain injury produces deficits in learning and memory in rats. (A) Spatial acquisition trials conducted on Days 21-26 post-injury revealed deficits in learning in single-injury animals on Days 22-24 and 26 in comparison to control. Repeat-injury was associated with deficits on Days 22-26 in comparison to control. No differences were seen between single- and repeat-injury paradigms. (B) The probe test on Day 27 revealed a statistically significant difference in time spent near the prior platform location between injured animals (single or repeat) and control animals. No difference was seen between single- and repetitively-injured rats. (C) Animals exposed to neurotrauma exhibited a trend towards increased thigmotaxis during spatial acquisition procedures but this finding did not reach statistical significance. 


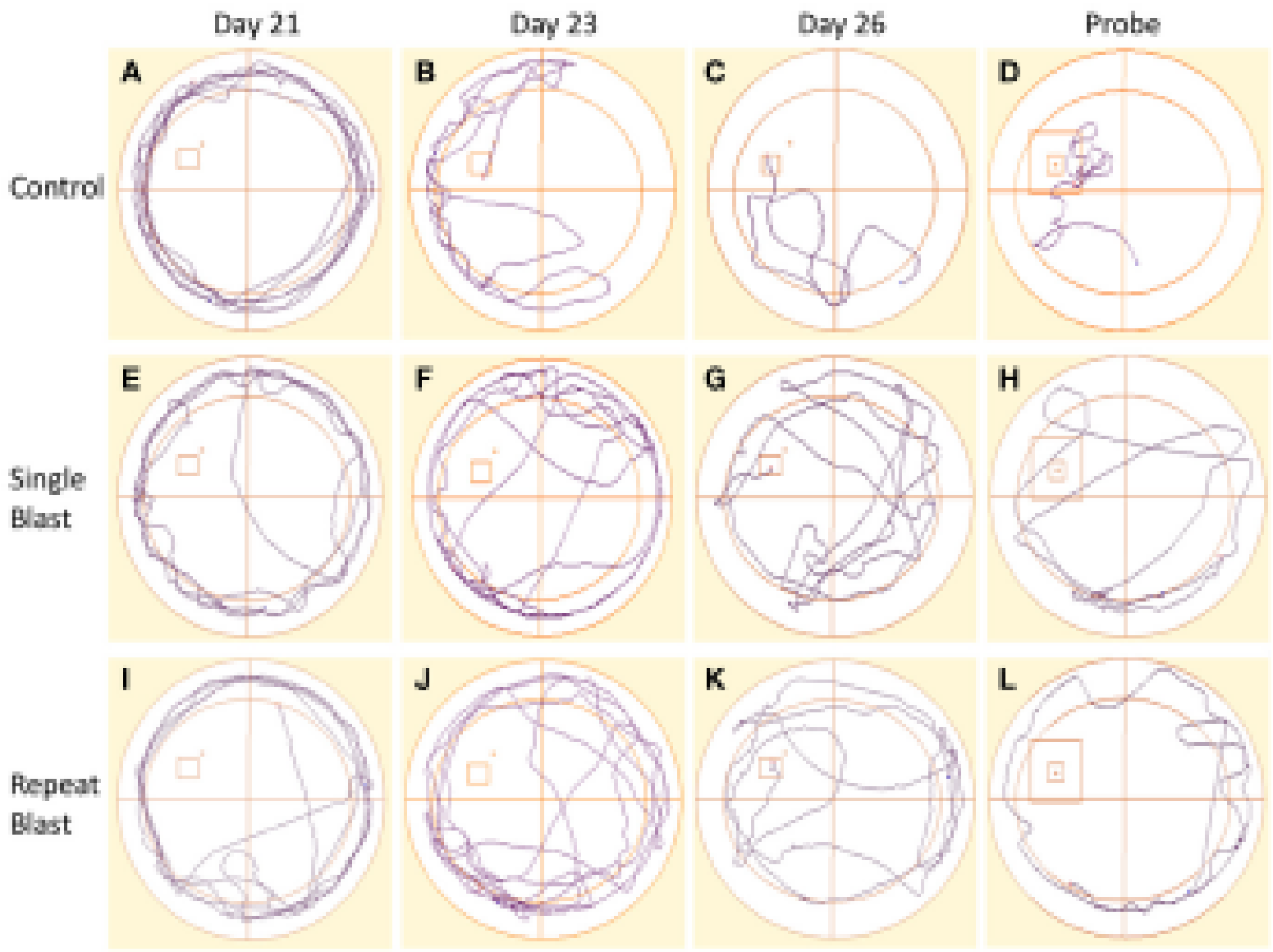

Figure 6.9. Track plots acquired across spatial acquisition and probe testing in rats. (A-D) Track plots generated from anesthetized control animals on Day 21, Day 23, Day 26, and during the probe test. (E-H) Track plots generated from single-injured animals on Day 21, Day 23, Day 26, and during the probe test post-injury. (I-L) Track plots generated from repetitively-injured animals on Day 21, Day 23, Day 26, and during the probe test post-injury. Injured animals appear to spend more time exhibiting thigmotaxis behaviors (circling edge of pool) on later days and in the probe trial fail to spend as much time as the anesthetized control animals within the probe region and the direct area surrounding the probe region. 

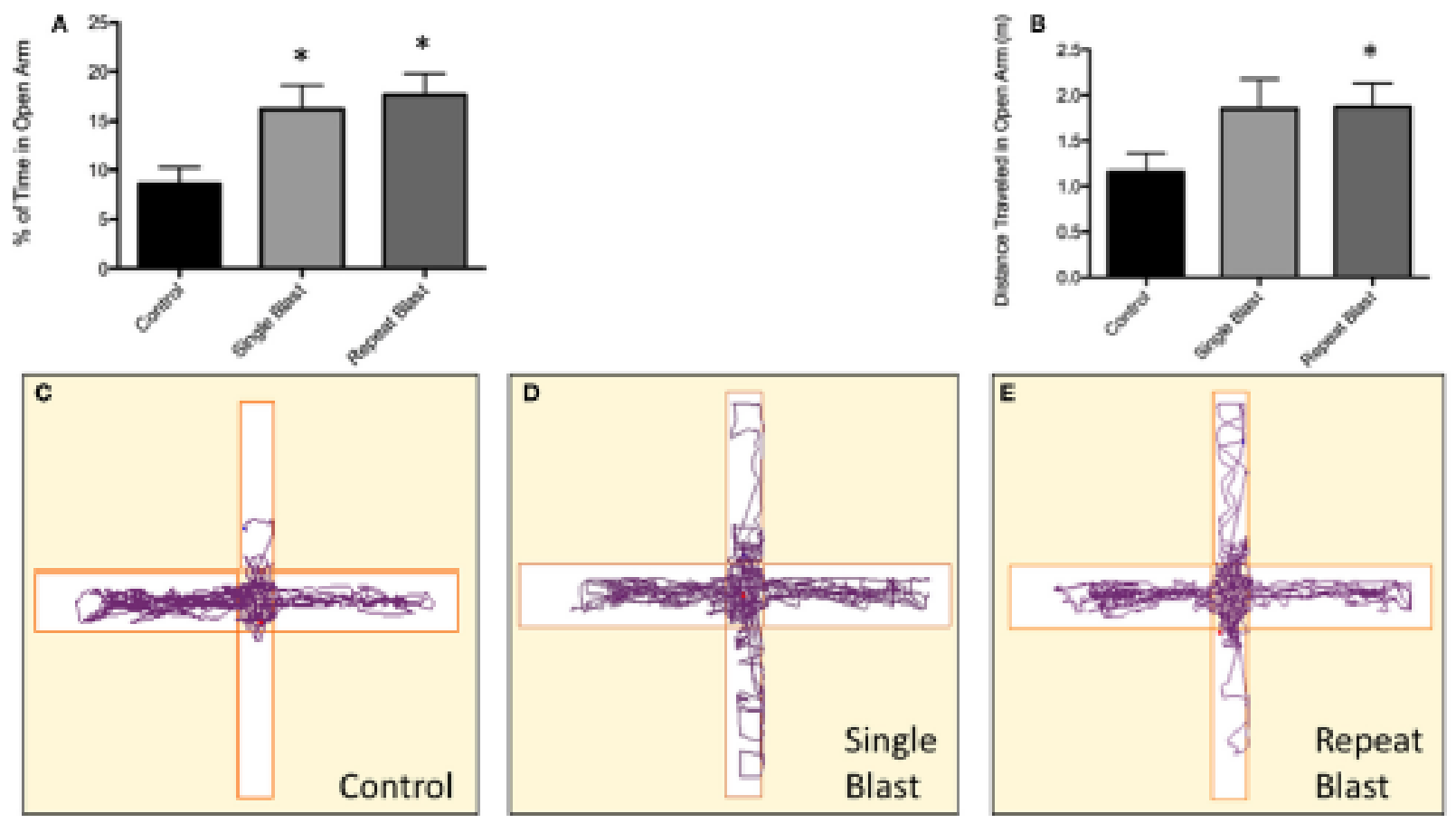

Figure 6.10. Blast-induced brain injury in rats produces an increase in impulsive behavior based on the elevated-plus maze. (A) A significant difference ( $p<0.05$ vs. CTRL) was seen between anesthetized control animals and both single and repeat blasts with regards to the percentage of time spent in the open arm of the elevated-plus maze. No difference was measured between single and repeat-injury paradigms. (B) While not reaching statistical significance, a trend towards greater distance traveled in open arms was seen with neurotrauma, a finding consistent with the documented increase in time spent in the open arms. (C) Animals subjected to neurotrauma appeared to travel less distance in the closed arms of the maze, although this finding did not reach statistical significance. (D) Track plot showing little exploration of the open arm by control animals. (E) Track plot showing increased exploration of the open arms of the elevatedplus maze by single-injured animals. (F) Track plot showing increased exploration of the open arms of the elevated-plus maze by repetitively injured animals. 


\section{Chapter Seven}

\section{Modeling Chronic Traumatic Encephalopathy: The Way Forward for Future Discovery}

This work is published in Front Neurol. 2015 Oct 26;6:223.

doi: 10.3389/fneur.2015.00223. eCollection 2015. Review. PMID: 26579067

Turner RC*, Lucke-Wold BP*, Logsdon AF*, Robson MJ, Lee JM, Bailes JE, Dashnaw ML, Huber JD, Petraglia AL, Rosen CL. 


\section{ABSTRACT}

Despite the extensive media coverage associated with the diagnosis of chronic traumatic encephalopathy (CTE), our fundamental understanding of the disease pathophysiology remains in its infancy. Only recently have scientific laboratories and personnel begun to explore CTE pathophysiology through the use of preclinical models of neurotrauma. Some studies have shown the ability to recapitulate some aspects of CTE in rodent models, through the use of various neuropathologic, biochemical, and/or behavioral assays. Many questions related to CTE development however remain unanswered. These include the role of impact severity, the time interval between impacts, the age at which impacts occur, and the total number of impacts sustained. Other important variables such as the location of impacts, character of impacts, and effect of environment/lifestyle and genetics also warrant further study. In this work we attempt to address some of these questions by exploring work previously completed using single and repetitive injury paradigms. Despite some models producing some deficits similar to CTE symptoms, it is clear that further studies are required to understand the development of neuropathological and neurobehavioral features consistent with CTE-like features in rodents. Specifically, acute and chronic studies are needed that characterize the development of tau-based pathology. 


\section{INTRODUCTION}

Corsellis described the original case series of chronic traumatic encephalopathy (CTE) in boxers (Corsellis et al., 1973). The disease consisted of brain atrophy, dilated ventricles, a cavum septum pellucidum, and pallor of the substantia nigra. Chronic traumatic encephalopathy was reintroduced into the medical lexicon by Dr. Bennet Omalu in 2005 (Omalu et al., 2005). Omalu described a progressive tauopathy that was seen in the brains of deceased football players. In recent years, McKee, Goldstein, and Stern have defined clinical and pathologic features of the disease. These include behavioral disturbances such as impulsivity, depression, and lack of oversight (Corsellis et al., 1973; Mez et al., 2013). Pathologic criteria include neurofibrillary tangles in a perivascular distribution and within superficial cortical areas with occasional amyloid and TDP43 protein aggregations (Baugh et al., 2014). Stern recently expanded the CTE criteria further by describing young vs. old onset based on symptom manifestation (Stern et al., 2013). Interestingly, blast traumatic brain injury has been linked to CTE following a single exposure, where athletes develop the disease following repetitive head injury (Lucke-Wold et al., 2014b).

CTE has been defined as a slowly progressive disease that takes years to decades to develop, often providing a significant latent period between when the neurotrauma occurs and when symptoms develop. A few cases involve athletes/soldiers as early as late teen's to early twenties (Omalu et al., 2011a; Tartaglia et al., 2014). The reason for the discrepancies in age of presentation observed is currently unknown but is likely due to the age at which impacts were sustained and the severity of the injury. Prior studies have shown that children with TBI have inadequate development of social cognition (Ryan et al., 2014) and that adolescents can develop post-traumatic headaches. Very rarely however do either of these groups experience symptoms of CTE (Dubrovsky et al., 2014). Early behavioral symptoms of CTE usually do not appear until the 
mid-thirties, and cognitive impairment does not begin until the early sixties (Baugh et al., 2014). Recent evidence suggests that neurotrauma may be linked to other neurodegenerative diseases such as Alzheimer's disease (AD) as well (Johnson et al., 2010).

CTE and AD, despite both being tauopathies, have generally been viewed as separate diseases. Each disease has distinct clinical presentations and unique clinical risk values. This view has gradually been changing with some reports showing that CTE can develop within the context of AD. The relationship between these two conditions however is poorly understood. Prior studies have indicated that TBI is a risk factor for development of cognitive impairment and AD (Guskiewicz et al., 2005), but whether the conditions are additive or synergistic remains unclear (Weber, 2007). Recent evidence suggests that neurotrauma may both increase the likelihood of disease development and accelerate the development of AD (Barnes et al., 2014). Notably, the wealth of AD pathology observed in preclinical neurotrauma models supports the idea of disease acceleration (Tran et al., 2011). In addition to AD, some groups have presented a possible link between CTE and a variant of amyotrophic lateral sclerosis that has been termed 'chronic traumatic encephalomyelopathy' (CTEM) (McKee et al., 2010). Therefore, it is clear that neurotrauma may have many lasting deleterious consequences, including the potential for increased risk and accelerated development of neurodegenerative diseases such as AD, CTE, and CTEM. While the data on these studies is preliminary in nature and not prospective, these findings demonstrate the need for further investigation with both preclinical models and clinical trials.

\section{The Quest for the Ideal Model}

The search for ideal preclinical models to study CTE remains an area of ongoing investigation with a relative paucity of prior studies. Of those published previously, the majority of studies fail to recapitulate the extensive neuropathologic and neurobehavioral aspects of injury 
(Goldstein et al., 2012; Ojo et al., 2015). Post-mortem identification of neurofibrillary tangles are a key diagnostic marker used clinically (Baugh et al., 2012). Therefore, the development of hyperphosphorylated tau in animals following injury must be an important component in establishing a CTE model. Tau hyperphosphorylation appears to correlate with the emergence of neuropathologic and neuropsychiatric deficits representative of neurotrauma related neurodegeneration (Petraglia et al., 2014a). By modeling CTE in rodents, we can better understand disease development and discover potential therapeutic avenues. Similarly, little justification is given to injury severity, number of impacts, interval between impacts, and age at which the impacts occur. Few studies have also evaluated secondary mechanisms of injury. In the following sections, we discuss the advancements and shortcomings of prior research while highlighting areas in need of further investigation.

\section{What are the proper controls for studying CTE?}

One of the challenges in creating a preclinical model for CTE is the establishment of proper controls comparing sham to TBI animals (Weber, 2007). Specifically, sham animals must undergo the exact same procedure each day of the injury paradigm. They must be anesthetized for the same duration as injured animals. The age of the animal must also be considered. Control animals should be the same age as experimental animals at time of sacrifice. This is an important consideration in designing extended studies with behavioral assays. Biochemical time courses also require control animals for each time point. Numerous questions must be addressed in planning experiments while considering the implications of each decision in terms of experimental question addressed and potential complications (Figure 1).

Utilizing Available Tools For Studying CTE 
One of the current weaknesses of clinical investigation of CTE is that the disease remains a neuropathological diagnosis. Some advancement has been made in ligand-based PET imaging. The imaging was used in a cohort of patients with an extensive history of neurotrauma, but the modality is not readily available (Small et al., 2013). Consequently, clinical observations are largely retrospective in nature and it is nearly impossible to investigate disease onset and progression. These clinical shortcomings can be readily addressed through the use of adequately designed preclinical models.

The first step in creating a preclinical model of CTE is to choose a model that can be used to generate a combination of biochemical and behavioral changes post-injury consistent with the CTE-like phenotype. Specifically, tauopathy or a precursor of tauopathy (tau hyperphosphorylation), must be present. The changes in tau must be inducible in genetically unaltered animals and then must be verified with transgenic rodents. Behavioral changes must be induced by neurotrauma. These changes should persist or worsen at chronic time points postinjury. Assessment of behavior should include tests capable of evaluating behavioral symptoms reported in clinical CTE cases. In particular, tests for cognition, depressive-like behavior, and impulsive-like behavior should be used. Cognition can be measured using Morris water maze (MWM) whereas depressive-like behavior can be assessed with the forced swim test or tail suspension test. Impulsivity can be elucidated with the elevated-plus maze (EPM).

Once a model is established, biochemical, electrophysiological, and advanced imaging techniques can be employed to assess neural injury. Biochemical mechanisms of potential interest include those related to cell survival/death, regulation of tau phosphorylation/dephosphorylation (kinases/phosphatases), bioenergetics, and propagation of tau-based changes. Electrophysiological studies can be performed to identify effects of injury and CTE development 
both on individual synapses and larger tracts such as the Schaffer collaterals within the hippocampus. Imaging modalities such as magnetic resonance imaging (MRI), functional MRI (fMRI), spectroscopy (MRS), and PET studies have the unique advantage of being able to be performed longitudinally with multiple assessments of the same animal at different time points. Taken together, integration of biochemical, behavioral, electrophysiological, and imaging modalities may provide insight into the mechanisms and time course of CTE development. This approach will also allow for more definitive evidence to be gathered. This evidence will provide a stepping-stone in addressing key questions about the effect of inter-injury interval, injury severity, and number of cumulative injuries necessary for the development of CTE. The answers garnered from this potential work may then influence the design of clinical trials that dictate return-to-play decisions. Baseline monitoring may become required in sports arenas and battlefields with high incidences of neurotrauma. The monitoring may assist in detecting cumulative subthreshold injury levels and be used to decrease the overall level of concussions.

\section{Implications for Modeling CTE: Results from Prior CTE Modeling Studies}

Perhaps one of the most promising studies, with regards to demonstrating CTE-like disease in a rodent model, was performed by Luo, et al. In this study, the authors developed a closed head model of neurotrauma utilizing an electromagnetic stereotaxic impact device. The authors showed that enhanced force of injury or using repeat injury increased GFAP-tagged luciferase. Intriguingly, when this same repeat injury paradigm was applied to wild-type mice, spatial learning and memory deficits were observed 2 to 6 months after injury and were accompanied by increased hyperphosphorylated tau and astrogliosis (Luo et al., 2014). The gliosis response in human CTE has not been well characterized, but the findings by Luo and colleagues show that GFAP was increased near areas of tauopathy. Similar findings have been seen by Petraglia and colleagues 
who performed a rigorous investigation of the behavioral effects of both single and repetitive closed head injury in wild-type mice (Petraglia et al., 2014b). The authors found that a single injury, in un-anesthetized animals, produced notable short-term abnormalities in behavior similar to a post-concussive state. Repetitive injury (42 impacts total: 6 impacts per day over 7 days) produced chronic deficits, particularly with regards to depressive-like and risk-taking behaviors as well as spatial learning and memory (Petraglia et al., 2014b). This same group published a recent follow-up study demonstrating the presence of hyperphosphorylated tau, a precursor of neurofibrillary tangle (NFT) development, in repetitively injured animals (Petraglia et al., 2014a). The model produces several of the same behavioral symptoms reported by patients suspected of having CTE. We recently show that endoplasmic reticulum stress contributes to tauopathy and CTE-like behavioral deficits following repeat blast injury (Lucke-Wold et al., 2015c).

Other studies, conducted by Mouzon and colleagues report both behavioral and neuropathologic changes following repetitive neurotrauma in mice. Specifically, 5 injuries administered 48 hours apart, produced durable cognitive deficits, learning disabilities, diminished rotarod performance, and changes in anxiety-like behavior on elevated-plus maze. Notably, these behavioral changes occurred in conjunction with persistent neuroinflammatory changes and disruption of white matter integrity. No changes in $A \beta$ and tau phosphorylation were seen at the chronic time points of 6 and 12 months post-neurotrauma, likely because rodents don't naturally develop neurofibrillary tangles (Mouzon et al., 2014). Rodents do however demonstrate acute tau changes due to phosphorylation and cleavage following injury (Huber et al., 2013). Liu and colleagues found that tau hyperphosphorylation was increased in rats acutely post-injury and triggers caspase activation in rat cortices (Liu et al., 2011). The activation of cell death can lead to circuit dysfunction and behavioral deficits (Abisambra and Scheff, 2014). Goldstein and 
colleagues show that tauopathy contributes to mitochondrial dysfunction and microtubule injury that ultimately leads to apoptosis. In this study, they found that tau modulation is a potential avenue for therapeutic intervention (Kondo et al., 2015). We recently report that caspase activation was increased in human CTE brains near sites of neurofibrillary tangle formation (Lucke-Wold et al., 2015a).

Other repeat-injury studies have investigated changes in tau and amyloid post-injury with the goal being to more clearly elucidate the relationship between TBI and neurotrauma related neurodegeneration. One report showed an increase in neuronal tau immunoreactivity (Kanayama et al., 1996) and another showed elevated amyloid precursor protein (APP) (Dobrowolska and Gibson, 2005; Weber, 2007) at a variety of time points post-injury. A final study by Zhang and colleagues showed that monoacylglycerol lipase can lead to behavioral deficits and tauopathy characteristic of a CTE-like phenotype (Zhang et al., 2015). These findings were further verified in other studies using transgenic models of amyloidosis and tauopathy in which repetitive injury paradigms produced elevated amyloid and tau levels with increased deposition. Single injury however failed to produce changes above control levels (Uryu et al., 2002; Conte et al., 2004). Using a T44 tau Tg mouse line, Yoshiyama and colleagues sought to study dementia pugilistica (DP), a condition sharing many features with modern day CTE, but with several key differences. DP by definition occurs only in boxers and has more severe gross anatomical changes that are not always present in CTE. The Yoshiyama group found that 4 impacts per day given once a week for 4 weeks produced only modest neuropathology with only 1 mouse demonstrating CTE or DP characteristics out of a total of $18 \mathrm{Tg}$ mice and 24 wild-type mice (Yoshiyama et al., 2005; Weber, 2007). The affected mouse displayed neuropathologic changes that included heightened tau burden, the presence of neurofibrillary tangles, and cognitive deficits (Yoshiyama et al., 2005). 
While it remains unclear why only one mouse developed such pathology, neuropathological findings from this one animal demonstrated that iron deposition was increased and associated with blood-brain barrier disruption. Iron deposition, associated with degradation of heme, activates oxidative stress-related pathways. Importantly, this oxidative stress is then associated with accelerated neurofibrillary tangle formation in these perivascular locales (Yoshiyama et al., 2005).

Notably, even single injury paradigms have been shown to produce tauopathy in a variety of injury models including blast (Goldstein et al., 2012), fluid percussion (Hawkins et al., 2013), and controlled cortical impact, findings consistent with prior clinical reports (Begum et al., 2014; Rubenstein et al., 2014). Goldstein and colleagues demonstrated that tauopathy following single blast injury was associated with hippocampal-dependent learning and memory deficits at subacute and chronic time points. These changes were also associated with electrophysiological alterations in long-term potentiation (Goldstein et al., 2012). Therefore, even a single mild to moderate injury may induce neurodegeneration and neurological deficits leading to impaired cognition and disrupted synaptic transmission (Goldstein et al., 2012). Single injury has also been shown to contribute to blood brain barrier disruption (Glushakova et al., 2014). This is a significant finding considering the numerous concussive and subconcussive injuries occurring in athletics and on the battlefield. Other studies using single injury models have also demonstrated activation of numerous pathological processes and behavioral changes associated with neurodegeneration. Modeling neurotrauma related neurodegeneration is a key component in the search for a model of CTE (Smith et al., 1995; Logsdon et al., 2014; Lucke-Wold et al., 2014c). While the importance of repeat injury in CTE modeling cannot be overstated, some single injury studies have led to advances in the ability to detect phospho-tau in serum at weeks to months post-injury. These advances indicate the potential role of biomarkers in monitoring and understanding disease 
pathophysiology (Rubenstein et al., 2014). The development of animal based models that exhibit similar characteristics of CTE will afford researchers the opportunity to characterize the acute and chronic effects of injury on the phosphorylation of tau in controlled experimental conditions. In vitro models may even be beneficial in elucidating changes at the cellular level (Zander et al., 2015). Evaluating imaging modalities, potential biomarkers, such as phosphorylated tau, and proposed therapeutics in a controlled context will promote advancement towards clinical applications and could be instrumental for monitoring and understanding disease pathophysiology in the future.

\section{Implications for Modeling CTE: Learning from Past Shortcomings with TBI Models}

Despite these notable advancements in CTE modeling as outlined above, the vast majority of repeat injury studies fail to address the role of injury severity, inter-injury interval, and the total number of impacts needed to reproduce a CTE-like state (Prins et al., 2010; Goddeyne et al., 2015). Most studies simply describe features of TBI without relating the findings to neurodegeneration (Loane and Kumar, 2015). Furthermore, because CTE is a neuropathologic diagnosis, any study claiming to serve as a model of CTE must demonstrate the hallmark neuropathologic changes (tau hyperphosphorylation and neurofibrillary tangles) and show that these changes persist at delayed time points and coexist with behavioral deficits. Few studies have looked at amyloid, tau, or TDP43 accumulation following injury.

One of the most common omissions from repetitive injury paradigms is the lack of consideration for inter-injury interval. Work by Longhi and colleagues directly explored this issue, demonstrating that in a mouse model of closed-head injury (CHI), mice had a period of vulnerability estimated between 3 and 5 days where the effect of injuries was additive and produced deficits in cognition. When the inter-injury interval was lengthened to 7 days, these 
deficits were not present (Longhi et al., 2005). While the focus of that study was elucidating differences in the inter-injury interval, other groups employing a repeat injury paradigm should consider how the inter-injury interval may relate to their findings. Inter-interval design will be an important consideration in developing CTE models. Attempting to provide a clinical context for these decisions would be desirable, with the interval between severe concussive-type impacts ideally being longer than that between mild subconcussive-type impacts. This would expand scientific insight for "return-to-play" guidelines that require players to be asymptomatic following a diagnosed concussion prior to returning to contact sports. These types of questions have generally not been considered in preclinical work, with most studies using brief inter-injury intervals, often ranging from minutes to 24 hours with poor justification for timing (Creeley et al., 2004; Shitaka et al., 2011; Genovese et al., 2013; Klemenhagen et al., 2013; Petraglia et al., 2014b; Wang et al., 2014).

Another important limitation related to interpretation of injury severity and neuropathologic outcome, is the sensitivity of detecting neuropathology using current scientific approaches. Shitaka and colleagues demonstrated previously that silver staining was more reliable for detecting axonal injury and pathology in comparison to routine histological analysis, assessment of neuronal cell loss, and amyloid precursor protein (APP) immunohistochemistry (Shitaka et al., 2011). It may be increasingly important, particularly in studies of repetitive subconcussive impacts, to utilize measures of high sensitivity for injury detection. Silver staining, which has been shown repeatedly to exhibit a higher degree of sensitivity in detecting axonal injury than many immunohistochemical techniques; electron microscopy; or other markers with these characteristics may prove promising (Shitaka et al., 2011). It has not yet been determined if neurofibrillary tangles accumulate around sites of axonal shearing. 


\section{Key Questions Going Forward}

Improving experimental models will enhance the quest for developing therapeutic agents that can be used to prevent and treat CTE. The search for a model of CTE raises a number of questions that are important clinically. These questions include issues such as length of the inter-injury interval, the number and severity of impacts, and the age at time of impacts, as well as the mechanism of impact, the gender of the patient, and what role genetic predisposition may play in the development of neurodegenerative disease following neurotrauma. Another important question that must be addressed is does a history of neurotrauma and potential presence of CTE accelerate the development of Alzheimer's disease? Furthermore, how can the period of susceptibility following neurotrauma be identified most readily? In the following sections we attempt to address these questions based upon available evidence. We also provide suggestions for handling shortcomings going forward. Studies upon which these sections are based are referenced in Tables 1-6 for quick reference.

\section{Inter-Injury Interval}

The effect of inter-injury interval on outcome following TBI has only recently been investigated. Studies have explored a variety of intervals ranging from 2 minutes apart (in vitro) to a few hours (in vivo) to as long as 30 days apart (in vivo). Using a novel approach assessing vasoreactivity in TBI, Fujita and colleagues demonstrated that administration of seemingly mild injuries at brief intervals ( 3 hours apart) produced dramatic declines in vasoreactivity and axonal pathology. When the inter-injury interval was lengthened to 5 hours, the magnitude of these changes was diminished substantially with complete dissolution of changes in both pathology and vasoreactivity at 10 hours (Fujita et al., 2012b). 
Other studies that have investigated longer inter-injury intervals have identified periods of susceptibility following an initial impact at periods ranging from 24 hours to a few weeks (Meehan et al., 2012; Mannix et al., 2013; Bolton and Saatman, 2014; Weil et al., 2014). Bolton and colleagues demonstrated with a CHI model that a single impact produced extensive gliosis bilaterally in the hippocampi and entorhinal cortices. Repeat injury after 24 hours produced a more severe injury consisting of hemorrhage in the entorhinal cortices as well as heightened measures of neurodegeneration, gliosis, and neuroinflammation (Bolton and Saatman, 2014). When the experimental paradigm was changed such that impacts were given with a 48-hour interinjury interval, the histopathology resembled that of a single impact suggesting enhanced susceptibility when a second impact was administered within 24 hours (Bolton and Saatman, 2014).

Mannix and colleagues performed one of the most rigorous investigations of the effect of inter-injury interval on outcomes associated with TBI. These studies included measures of both cognition and neuropathology related to the development of neurodegenerative diseases such as CTE and AD. In this study, the investigators showed that animals who received daily or weekly injuries with weight-drop had persistent cognitive deficits up to 1 year post-injury (Mannix et al., 2013). This was in contrast to when animals were injured biweekly or monthly, which failed to produce deficits at such a chronic time point (Mannix et al., 2013). Notably, the cognitive deficits seen in the daily- and weekly-injured animals did not correlate with elevations in tau phosphorylation or amyloid- $\beta$ when measured by ELISA nor brain volume loss when measured by MRI (Mannix et al., 2013). This finding may indicate that in addition to inter-interval time, injury severity must be considered. Tauopathy is essential for modeling CTE therefore an appropriate inter-injury interval might be best characterized in transgenic animals. Weight drop 
produces variable injury based on the height of the drop. A more severe TBI can produce cognitive deficits but may not be representative of the concussive and subconcussive injuries associated with CTE. A mild TBI with a transgenic animal will likely produce the most relevant deficits.

Meehan and colleagues performed a similar study with primarily behavioral assays. The investigators subjected mice to a $\mathrm{CHI}$ via weight-drop for a total of 5 impacts at various intervals. These intervals included daily, weekly, and monthly intervals. Mice receiving 5 impacts total at daily or weekly intervals were impaired in the MWM compared to sham animals (Meehan et al., 2012). This was not the case when injuries were delivered at monthly intervals, as these animals exhibited no impairment in the MWM (Meehan et al., 2012). Interestingly, at one month postinjury, the daily- or weekly-injured animals still exhibited deficits in the MWM and this deficit persisted in daily-injured animals out to 1 year (Meehan et al., 2012). This finding may represent why football lineman who experience daily subconcussive injuries appear more likely to develop CTE based on the clinical cases reported. It is still necessary to establish if tauopathy is the driving mechanism behind behavior. The findings confirm and expand upon the inter-interval studies completed by Longhi and colleagues (Longhi et al., 2005). Longhi reported that shorter interinterval injuries produce worse outcomes, which is in agreement with the Mannix and Meehan findings. These studies were consistent with findings in higher phylogenetic species as well, specifically piglets. Friess and colleagues showed that a 24 -hour inter-injury interval produced more severe deficits and higher mortality rates than when the interval was extended to 7 days (Friess et al., 2009). Finally, Kanayama and colleagues demonstrated a graded response in locomotor activity. Both shorter inter-injury intervals as well as greater number of total injuries were associated with worse outcome (Kanayama et al., 1996). 
Weil and colleagues explored the effect of altering the inter-injury interval in relation to recovery from TBI. They used a clear clinical-minded approach and utilization of metabolic imaging (PET). This group showed that injuries separated by only 3 days were associated with worse neuropathology and an inability to mount the typical hypermetabolic response with regards to glucose utilization following TBI. This worse outcome was not seen following either a single injury or repeat injuries with an extended inter-injury interval of 20 days (Weil et al., 2014). Similarly, a brief inter-injury interval of 3 days was associated with elevated IL- $1 \beta$ and TNF $\alpha$ gene expression when compared with other experimental groups (Weil et al., 2014).

The longest interval between injuries used in preclinical studies, to the best knowledge of the authors, was 30 days. The additional injury had no additive effect on anxiety (EPM), depression (FST), and cognitive function (MWM) when compared to animals receiving only one injury (Mychasiuk et al., 2014). These findings indicate that either the window of vulnerability following the first injury was avoided or that the response to the first injury may protect the animal from subsequent injuries, a concept known as preconditioning (Mychasiuk et al., 2014). The progression towards tauopathy was not well characterized in this work.

In vitro studies have shown similar findings to the in vivo studies described above. Shorter inter-injury intervals between mechanical stretching resulted in an elevation in S-100 $\beta$ protein release and increased cellular permeability identified with propidium iodide staining (Weber, 2007). Similarly, a 'subthreshold' level of stretch did not produce any overt cellular damage or death when repeated at 1-hour intervals but did cause neuronal loss and neuron-specific enolase (NSE) release when performed at incredibly short intervals (every 2 minutes) (Slemmer and Weber, 2005; Weber, 2007). Remarkably, this rapid and repetitive 'subthreshold' stretch that produced changes in neurons, failed to produce an increase in S-100ß protein release, indicating a 
differential response between neurons and glia to neurotrauma severity and interval (Slemmer and Weber, 2005; Weber, 2007).

In contrast to the above studies, one group showed that repeat injury, when administered in different anatomical locations within the brain, failed to result in heightened damage when an inter-injury interval was 3 days. It did however increase tissue vulnerability with a 7-day interval as evident by increased hemorrhage (Donovan et al., 2012; Donovan et al., 2014). The authors of the study therefore argue that the period of susceptibility likely depends on not only the time interval between injuries, but also the anatomic location of injury (Donovan et al., 2012; Donovan et al., 2014). Importantly, this study utilized an open injury model (controlled cortical impact), a scenario that is only seen in a subset of clinical neurotraumas.

In summary, these studies demonstrate a period of vulnerability following initial injury in which sustaining a second brain injury may result in an additive effect. Additive injury is not clearly apparent when the brain is allowed a more extensive recovery period. Interestingly, this vulnerability may not solely be due to initial axonal pathology but may also be the result of cerebrovascular reactivity and the inability to utilize glucose effectively (Fujita et al., 2012b; Weil et al., 2014). This concept of a varied cellular response to TBI is consistent with findings from in vitro studies that demonstrate a varied response amongst glia and neurons (Slemmer et al., 2004; Slemmer and Weber, 2005; Weber, 2007).

\section{Number of Impacts}

The prevailing theory for 'mild' neurotrauma is that repetitive injuries are associated with more short- and long-term detrimental effects than a single injury alone (Laurer et al., 2001; Mouzon et al., 2012; Bolton and Saatman, 2014). This concept has been applied regardless of injury severity (concussive versus subconcussive) with emerging evidence indicating that even 
subthreshold impacts are cumulatively detrimental (Fujita et al., 2012b; Bailes et al., 2013). In this section we explore the effect of repeat injury through analysis of studies employing both repeat and single injury paradigms.

One of these studies, conducted by Mouzon and colleagues, showed that when rodents are exposed to 5 total injuries with an interval of 48 hours, these animals exhibit both impaired learning and memory at extended time points (Mouzon et al., 2014). These findings are in contrast to single injured animals that display only learning deficits but no retention impairment at the same time points (Mouzon et al., 2014). This study closely parallels the clinical findings documented by Guskiewicz and colleagues in which former athletes with a history of repetitive concussions experience memory-related issues at a rate 5 times higher than those without a history of concussion (Guskiewicz et al., 2005; Mouzon et al., 2014).

Other work that investigated various iterations of impacts $(0,1,3,5$, and 10$)$ demonstrated that while a single injury does not produce deficits in MWM performance in comparison to shaminjured animals, repetitive injury does in fact produce deficits and these deficits may exhibit a dose-dependent relationship. When mice were given 10 concussive weight-drop injuries, those in which the weight was dropped from a height of 42 inches performed worse than those injured from a height of 38 inches. Therefore, this work demonstrates the potential for both injury number and injury severity in contributing to neurological dysfunction (Meehan et al., 2012). Luo and colleagues utilized a GFAP-driven luciferase mouse line and a repetitive closed head injury model to investigate cumulative decline. The investigators showed that there appeared to be a linear increase in GFAP luminescence from 1 to 3 injuries but that this response appeared to reach a plateau by 5 injuries (Luo et al., 2014). In addition to the increase in GFAP fluorescence with repetitive injuries, mice receiving three injuries demonstrated less freezing time than sham animals 
in both cued and contextual fear conditioning (Luo et al., 2014). This was in contrast to single injury animals that did not differ from sham-injured animals in cued or contextual memory (Luo et al., 2014). Consequently, this data demonstrates that an increase in injury number is associated with an increasing severity of injury markers (based on protein expression of GFAP) as well as functional deficits (fear conditioning). Others employing both single and repeat injury paradigms have shown that while single injury may not induce pathological findings, repeat injury, of the same severity, does. This was particularly notable in work by Uryu and colleagues in which repetitive injury produced an increase in $A \beta$ deposition in $\operatorname{Tg} 2576$ animals, whereas no increase was observed in single injury paradigms in these same animals (Uryu et al., 2002). Likewise, Kanayama and colleagues demonstrated that repeat injury paradigms induced tau hyperphosphorylation, a precursor to NFT formation, in conditions such as CTE and AD (Kanayama et al., 1996).

The observed findings in closed-head, repeat injury models, were also consistent with those found in open-head injury using a craniectomy and controlled cortical impact. In work by Donovan and colleagues, the investigators showed that repeat injury induces progressive and evolving changes that are not observed in single injury paradigms (Donovan et al., 2014). The basis of many of the memory and cognitive changes following repeat TBI, may be explained by electrophysiological alterations in synaptic transmission. In work by Aungst and colleagues, repetitive TBI was found to prevent the induction of long-term potentiation (LTP) 28-days postinjury due to alterations in the NMDA receptor. This is in stark contrast to single injury paradigms that revealed the ability to induce LTP in both hemispheres, with the contralateral hemisphere exhibiting less LTP than the ipsilateral hemisphere. The impairments in excitatory neurotransmission following repeat injury were accompanied by extensive neuroinflammation and 
neurodegeneration as well as behavioral/functional impairments (Aungst et al., 2014). Specifically, repeat injury produced deficits more severe than single injury when measured at oneweek intervals out to a month. Importantly, even single injury produced deficits at chronic time points post-injury when compared to sham animals, indicating long-term effects of TBI (Aungst et al., 2014).

Povlishock and colleagues expanded the concept of subthreshold injury. In these studies, the investigators showed that administering a single weight-drop injury from $1.0 \mathrm{~m}$ resulted in neither axonal nor microvascular change. With repeat injury of short inter-injury intervals (hours), significant axonal and microvascular pathology was observed (Fujita et al., 2012b). This work was the first to assess microvascular reactivity to acetylcholine (ACh) following repetitive subthreshold brain injury. This work demonstrates the clear danger of subthreshold impacts when sustained in a repeated and rapid fashion. It also illustrates the role of the microvasculature in neuronal injury, showing that the neurovascular unit is essential for neuronal homeostasis.

Importantly, work regarding the number of impacts has been extended higher up the phylogenetic tree to rabbits and piglets (Olsson et al., 1971; Raghupathi et al., 2004). In rabbits, repeated loading with loads of up to 1.5 atm failed to produce an additive concussive response over a single load. A multi-loading paradigm at higher loads however caused respiratory arrest (Olsson et al., 1971). In piglets, multiple less severe injuries induced neuropathological findings similar to a severe load based on the density of injured axons as well as number and distribution of foci (Raghupathi et al., 2004).

In vitro studies have reported similar findings to the in vivo studies. Weber and colleagues applied a mild stretch to hippocampal neuronal cultures that produced low-grade injury when applied at a single time. When this injury was repeated, the cells exhibited cumulative damage 
with two injuries inducing an increase in neuron-specific enolase (NSE) (Slemmer et al., 2004; Slemmer and Weber, 2005; Weber, 2007).

Notably, the group led by Mychasiuk and colleagues provides evidence contrary to the widely held belief that an increased number of impacts are associated with detrimental findings on behavioral or histological measures. With a 30-day inter-injury interval, rodents receiving multiple injuries performed similar to single-injury animals on measures of anxiety, depression, and cognitive ability. Therefore, they propose that receiving head injury at an early age may prime the brain to be less susceptible to the effects of a later neurotrauma, a theory known as 'preconditioning' (Mychasiuk et al., 2014). DeRoss, et al also showed that while one concussive impact resulted in diminished performance in $85 \%$ of animals, less deviation was seen with subsequent impacts. The number of impacts has an inverse relationship with animal performance in the water maze (DeRoss et al., 2002). Again, the mechanism behind these findings is not entirely clear as those animals sustaining multiple injuries also received additional exposure to the water maze, allowing for enhanced training/learning of the maze (DeRoss et al., 2002). Therefore, while repetitively injured animals did better than single-injured animals in the maze, this is likely a product of increased training rather than a protective response but this cannot be said with absolute certainty.

\section{Severity of Impacts}

The effect of injury severity on likelihood of neurodegenerative disease development is not entirely clear, although some clinical reports indicate that more severe injury results in a greater predisposition for AD development (Plassman et al., 2000; Uryu et al., 2002). What is known from preclinical studies using an array of animal models is that there is a dose-dependent increase in neural injury markers and cognitive deficits with more severe injury (DeFord et al., 2002; 
Manley et al., 2006; Long et al., 2009; Budde et al., 2013; Turner et al., 2013b; Luo et al., 2014). Similarly, repetitive 'mild' injuries may produce a phenotype more consistent with a single more severe injury (DeFord et al., 2002).

How 'mild' TBI contributes to the likelihood of developing CTE remains unclear. Emerging evidence from preclinical studies raises concern about lasting effects of subthreshold injuries when sustained in a rapid and repetitive fashion. These repetitive injuries contribute to vascular reactivity and subsequent axonal degeneration in vivo (Fujita et al., 2012b). Some studies suggest that severity of injury may dictate the rest period required to minimize cumulative cognitive deficits, although further studies are required to validate these findings (Meehan et al., 2012).

Similarly, preclinical studies, even those in which only a mild force is imparted to a thinned cranium, indicate that a substantive inflammatory response is produced quickly after injury (Roth et al., 2014). This response is associated with heightened vascular permeability, also seen clinically, as well as microglial response (Roth et al., 2014). It is these mechanisms, both primary and secondary, that may contribute to neurodegenerative disease post-neurotrauma.

In vitro studies may be of further use in addressing the role of injury severity, particularly in subconcussive/subthreshold-type studies, as levels of injury and subsequent cellular responses can be monitored rapidly and performed over a greater number of iterations at a lower cost in comparison to in vivo studies. In fact, a number of injury paradigms and mechanisms have been investigated successfully in this manner including fluid pulse-induced shear stress, repetitive stretching, and other mechanical deformation procedures, both in vitro and ex vivo (LaPlaca and Thibault, 1998; Mukhin et al., 1998; Sieg et al., 1999; Slemmer et al., 2004; Slemmer and Weber, 
2005; Morrison et al., 2006). The in vitro shearing studies found a significant amount of axonal beading and glial death (LaPlaca et al., 2005).

\section{Age at Time of Impacts}

Age is the biggest risk factor for the development of neurodegenerative disease and has been associated with poor outcomes following TBI in a variety of clinical and preclinical reports (Hamm et al., 1991; Maughan et al., 2000; Hoane et al., 2004; Anderson et al., 2009; Wali et al., 2011; Mehan and Strauss, 2012; Timaru-Kast et al., 2012; Turner et al., 2012d; Turner et al., 2014). Similarly, neurotrauma has been associated with an increased risk of neurodegenerative disease development with regards to AD (Plassman et al., 2000; Van Den Heuvel et al., 2007; Magnoni and Brody, 2010; Sivanandam and Thakur, 2012), PD (Bower et al., 2003), and CTE (Omalu et al., 2005; Omalu et al., 2006; McKee et al., 2010; Omalu et al., 2010b; Omalu et al., 2010a; Omalu et al., 2010c; Gavett et al., 2011; Omalu et al., 2011a; Omalu et al., 2011b; Goldstein et al., 2012; Stern et al., 2013; Montenigro et al., 2014; Omalu, 2014). One of the primary questions currently in the field is how the age at which the patient sustains the neurotrauma pertains to the development, or lack thereof, of CTE.

Similar findings have been observed in preclinical studies. Mychasiuk and colleagues showed that TBI during brain development leads to worse outcomes than TBI affecting the mature brain (Mychasiuk et al., 2015). Preclinical work has also shown that young animals experience

less edema than middle-aged animals following TBI (Kasturi and Stein, 2009). The increased edema is associated with an increase in lesion size in aged rodents experiencing TBI (Kumar et al., 2013). TBI in aged rodents is also more likely to increase sensorimotor and cognitive decline (Hoane et al., 2004). TBI in youth may ultimately be more detrimental for social development, whereas severe injury in the elderly results in rapid cognitive decline due to increased edema and 
therefore, lesion size. In regards to human TBI, it is unclear if the elderly would have a more progressive form of the disease similar to rodent studies or if the disease would develop in the normal manner. It is also important to consider that males $<35$ years old are the most likely to have repetitive TBIs, a group associated with heavy participation in sports and now the military (Theadom et al., 2014). Future preclinical studies should therefore continue to investigate TBI secondary mechanisms in both young and aged animals with particular attention paid to addressing repetitive injury paradigms and the development of CTE-like features, both behaviorally and biochemically.

\section{Mechanism of Impact}

CTE has been diagnosed in athletes sustaining direct impacts as a result of participation in warfare, football, wrestling, and soccer (Omalu et al., 2005; Omalu et al., 2006; McKee et al., 2010; Omalu et al., 2010b; Omalu et al., 2010a; Omalu et al., 2010c; Gavett et al., 2011; Omalu et al., 2011a; Omalu et al., 2011b; Goldstein et al., 2012; Stern et al., 2013; Montenigro et al., 2014; Omalu, 2014). The mechanism of injury is different between blast and athletic concussions, but how these mechanisms relate to injury progression remains to be elucidated. For blast, primary to quaternary injury must be considered (Kobeissy et al., 2013). Other important questions include what is the influence of linear versus rotational impacts? What is the effect of direct impacts such as a football tackle versus indirect impacts such as primary blast exposure? Furthermore, how can comparisons most accurately be made across these various impacts? Are accelerometers and recording systems (such as the HITS system) the best method for understanding and comparing these impacts? What role does high-speed videography and subsequent kinematic analysis play? Each of these questions remains to be answered and may provide further insight into understanding the role that impact type plays in CTE development. 


\section{Biochemical Mechanisms}

Interestingly, glymphatic clearance has recently been shown to play a role in injury progression. Iliff and colleagues showed increased tauopathy accumulation in aquaporin knockout mice following traumatic brain injury due to disrupted glymphatic clearance (Iliff et al., 2014). It has yet to be determined how the primary injury mechanism causes the disruption to glymphatic channels. Cernak proposed an interesting theory about low-frequency stress waves transmitting kinetic energy through tissue (Cernak, 2015). This mechanism may account for the dysfunction of the aquaporin channels. The energy transfer may also injure other cellular components such as axons or vessels. Chodobski and colleagues have shown that kinetic transfer of energy can account for blood brain barrier disruption (Chodobski et al., 2011). Blood brain barrier disruption postinjury can trigger increased neuroinflammation. Agoston and colleagues showed that blast traumatic brain injury, in particular, causes persistent neuroinflammation, which leads to behavioral deficits (Kovesdi et al., 2012). The neuroinflammation can also contribute to posttraumatic epilepsy and tauopathy (Salazar and Grafman, 2015). Investigating how tauopathy spreads is a topic warranting further investigation (Koliatsos and $\mathrm{Xu}, 2015$ ). Furthermore, Kobeissy and colleagues highlight in their recent review that underlying neuronal damage can cause lasting neuropsychiatric deficits such as post-concussion syndrome and post-traumatic stress disorder (Kobeissy et al., 2013).

\section{Role of Genetics}

Genetics and lifestyle choices may play a role in likelihood of sustaining a TBI and also the outcome following a TBI. Little to no evidence currently exists regarding lifestyle choices associated with TBI and only recently has genetic contribution to CTE been addressed. Specifically, it is well known in the human literature that the APOe4 allele is associated with worse 
outcome following TBI (Ponsford et al., 2011). APO 84 and APO $\varepsilon 3$ have also been implicated in the development of both CTE and AD (Weber, 2007; Saulle and Greenwald, 2012). The mechanism by which APOE4 worsens outcome following TBI is poorly defined (Mannix et al., 2013), but targeted replacement of the allele in mice has allowed focused research into cholesterol metabolism and may lead to insights in the field of traumatic brain injury and subsequent neurodegeneration (Laskowitz and Dawson, 2014). Notably, a study by Maroon and colleagues recently demonstrated that there was no significant difference between ApoE4 carriers in a population of patients afflicted with CTE when compared to the general population, suggesting that perhaps ApoE may not represent a significant risk factor for CTE development, even in individuals exposed to neurotrauma (Maroon et al., 2015).

Other genetic influences have also been observed but are currently limited to preclinical evidence. Rare genetic alterations such as mutation of the CACNAIA calcium subunit gene have also been shown to lead to poor TBI outcomes in human patients (Kors et al., 2001). Recent preclinical studies have shed light on other potential genetic factors that may influence TBI outcomes. Decreases in micro RNA 23a and 27a increase apoptosis following TBI in rodents (Sabirzhanov et al., 2014). Deficient caveolin expression can exacerbate neuroinflammation post-TBI (Niesman et al., 2014). The knock-in mouse APP696swe has accelerated deposition of A $\beta$ following TBI (Yoshiyama et al., 2005). Emerging evidence also suggests dysfunctional mitochondrial genes following TBI such as Fas, Apafl, and Chp. Interestingly, these genes become more dysfunctional and mutated with time after mild TBI (Sharma et al., 2012). TBI can also induce DNA fragmentation leading to an upregulation of p53, a critical regulator of cell cycle (Lu et al., 2000). On the other hand, upregulation of insulin growth factor expression prior to TBI is associated with neuroprotection (Carlson et al., 2014). Similarly, genetic regulation of aquaporin 4 channels can 
reduce edema formation following TBI in rodent models (Ke et al., 2001). Surprisingly, disruption or the PARP1 gene offers protection against TBI hypoxia (Hagberg et al., 2004). Studying genetic risk factors for TBI is an area of growing importance and requires further investigation. Understanding factors that lead to increased or decreased TBI severity may also allow the development of novel pharmaceutics for the prevention of neurodegenerative disease.

An emerging area is the role of epigenetic modulation following TBI. Epigenetic markers are now being used preliminarily to predict recovery following injury (Lipsky and Lin, 2015). VandeVord and colleagues show enhanced methylation of DNA in the rat hippocampus following blast traumatic brain injury (Bailey et al., 2015b). These epigenetic changes are mediated by HDACs and DNMTs (Bailey et al., 2015a). Interestingly, the methylation changes are cumulative with repetitive injury (Haghighi et al., 2015). HDAC has been shown to contribute to GSK3 $\beta$ activation, which is a known tau kinase. When HDAC is inhibited white matter damage is reduced (Wang et al., 2015). Epigenetic regulation has been tied to the development of post-traumatic stress disorder clinically (Almli et al., 2015). Targeting epigenetic regulation may therefore be a viable target in preventing tauopathy and behavioral deficits following traumatic brain injury.

\section{Influence of Gender}

The influence of gender on outcome after TBI remains controversial, particularly in light of the few cases of CTE diagnosed in women. Some studies claim that females have better outcomes following neural injury (Bramlett and Dietrich, 2001) while others report no change (Kadyan et al., 2004; Bruce-Keller et al., 2007), or worse outcome (Farace and Alves, 2000b). Estrogen treatment has shown improved outcomes in rodent models of neural injury (Simpkins et al., 1997; Suzuki et al., 2007; Jia et al., 2009; Li et al., 2011), including TBI (Gatson et al., 2012; Day et al., 2013). Female rodents exhibit better outcomes after neural injury as evidenced by 
increased neurotrophin production (Gatson et al., 2012), decreased neuroinflammation (Brown et al., 2009), and better performance on motor tasks (Wagner et al., 2004b). Clinical evidence shows that female patients exhibit lower oxidative damage after TBI (Wagner et al., 2004a), which could be the result of a higher estrogen circulation following injury. Gender differences should be considered when conducting clinical trials for TBI therapy.

Interestingly, Dixon and colleagues found that TBI in female humans reduces estradiol in the CSF (Garringer et al., 2013). The reduction of estradiol may have unique long-term effects because it is not yet known if this is transient or permanent. The limited amount of data about women with TBI has restricted the comparisons of injury between genders (Bell and Pepping, 2001). The majority of traumatic brain injuries in women are subdural hematomas from falls. Elderly women with subdural hematomas tend to fair worse than the general TBI population as a whole (Farace and Alves, 2000a). Menopause may therefore dampen the neuroprotective physiologic properties mediated by estrogen post-injury. TBI in elderly women is also linked with earlier onset Alzheimer's disease (Nemetz et al., 1999). Hormonal changes may also be a contributing factor to CTE, but this has yet to be verified. It is clear based on the limited preclinical and clinical data that further investigation into the gender differences surrounding TBI outcome is warranted.

\section{Effect of Environment}

Another area of investigation required for elucidation of factors influencing the likelihood and/or severity of CTE development following neurotrauma includes the effect of environment. Areas of particular interest include social support, diet, use of supplements, and use of drugs or anabolic steroids. 
Good social support has been shown to decrease the likelihood of developing a postconcussional disorder following acute head injury (McCauley et al., 2001). Strong family support is linked to better outcomes (Khan et al., 2003). Another important feature of environment is diet. While the effect of diet is well understood with regards to general health, there are limited studies relating diet and mental health, particularly in the context of traumatic brain injury. A preclinical study conducted by Mychasiuk and colleagues showed that high-fat diet in conjunction with TBI resulted in cumulative deficits on assessments of motor function, short-term working memory, and produced depressive-like effects compared to animals with normal diet and TBI (Mychasiuk et al., 2014). Similarly, administration of dietary supplements such as Vitamin E and DHA have been shown to improve outcomes from TBI in numerous studies (Conte et al., 2004; Wu et al., 2004; Mills et al., 2011b; Mills et al., 2011a; Bailes and Patel, 2014). The suggestion has been made previously, based on evidence that some cases of diagnosed CTE occurred in former athletes with a history of anabolic steroid use, that anabolic steroid use may predispose these athletes to CTE development. While this question has not been completely resolved in terms of studying markers of CTE, preliminary studies found no difference in amyloid precursor protein (APP) expression post-TBI regardless of when anabolic steroids were used (Mills et al., 2012).

\section{Preconditioning}

Any neural injury model has the potential to be complicated by the concept of preconditioning. Preconditioning at the most basic levels refers to neuroprotection for a given injury induced by a prior stimulus/injury. The concept of preconditioning has been well documented in a variety of neural injury models ranging from ischemic stroke to TBI (Weber, 2007). Initial stimuli that serve a protective effect in a subsequent injury include but are not limited to brief periods of ischemia, chronic exposure to moderate heat or heat acclimation, and 
subthreshold or mild injury (Weber, 2007). The proposed mechanism is that neuronal antioxidant machinery is upregulated with subthreshold injury thereby increasing the cells ability to respond to free radical production during subsequent injuries (Hu et al., 2008) Whether preconditioning serves a protective phenomenon in the development of CTE is unclear, but the general clinical consensus is that any brain injury no matter how small may be detrimental long-term, minimizing any potential benefit of the preconditioning phenomenon (Bailes et al., 2013; Talavage et al., 2014). This is in contrast to preclinical literature demonstrating that sustaining repetitive mild injury prior to a single severe injury protects the animal from the most deleterious effects of the severe injury (Allen et al., 2000). In other words, animals receiving repetitive mild injury prior to a severe injury do better than animals receiving a single severe injury (Allen et al., 2000). Similar findings have been observed in vitro in which subthreshold stretch prevented more deleterious injury when a threshold stimulus was given (Slemmer and Weber, 2005). The concept of preconditioning versus additive injury is captured pictorially in Figure 2.

\section{Summary}

In conclusion, understanding CTE as a disease remains in its infancy and current studies remain largely speculative in nature without prospective clinical investigation. The required clinical studies to advance the field mandate extensive financial resources and time. Preclinical studies represent the most promising mechanism for studying many of the basic biologic questions about CTE, as discussed above. While these studies are continuing to evolve, numerous groups have reported exciting findings. Better modeling has allowed more extensive biochemical and behavioral characteristics to be defined. Now that our laboratory and others have established CTE models, options for translational investigation of CTE pathophysiology abound. In this work we discussed numerous avenues for addressing translational questions, namely the role of 1) inter- 
injury interval, 2) number of impacts, 3) impact severity, 4) age at time of impacts, 5) mechanism of impact, 6) genetics, 7) gender, and 8) effect of environment on the development of CTE. We also highlighted some of the challenges of CTE modeling and specific requirements for successful models. By improving our understanding about CTE mechanisms, we believe that significant strides can be made not only in understanding CTE but also potentially developing prevention and therapeutic-related approaches. A companion manuscript describes our collective experience in modeling CTE, both neuropathologically and behaviorally. 


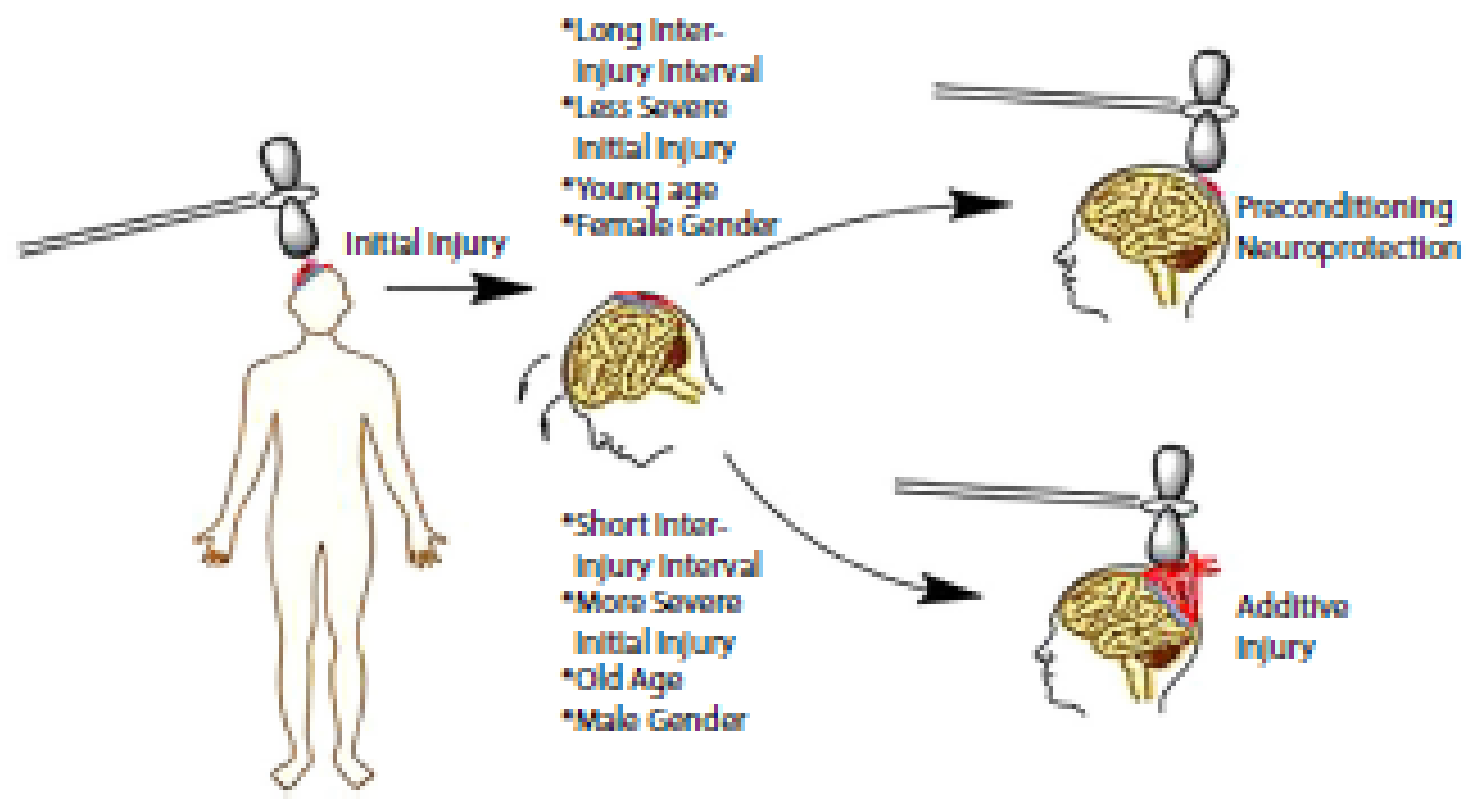

Figure 7.1. Schematic representation of factors influencing injury outcomes, particularly in repetitive injury paradigms. Initial evidence indicates that longer inter-injury intervals, less severe initial injury, a younger age, and female gender may serve as protective effects in repeat paradigms. In contrast, shorter intervals, increased severity, older age, and male gender may be associated with worse outcomes. 


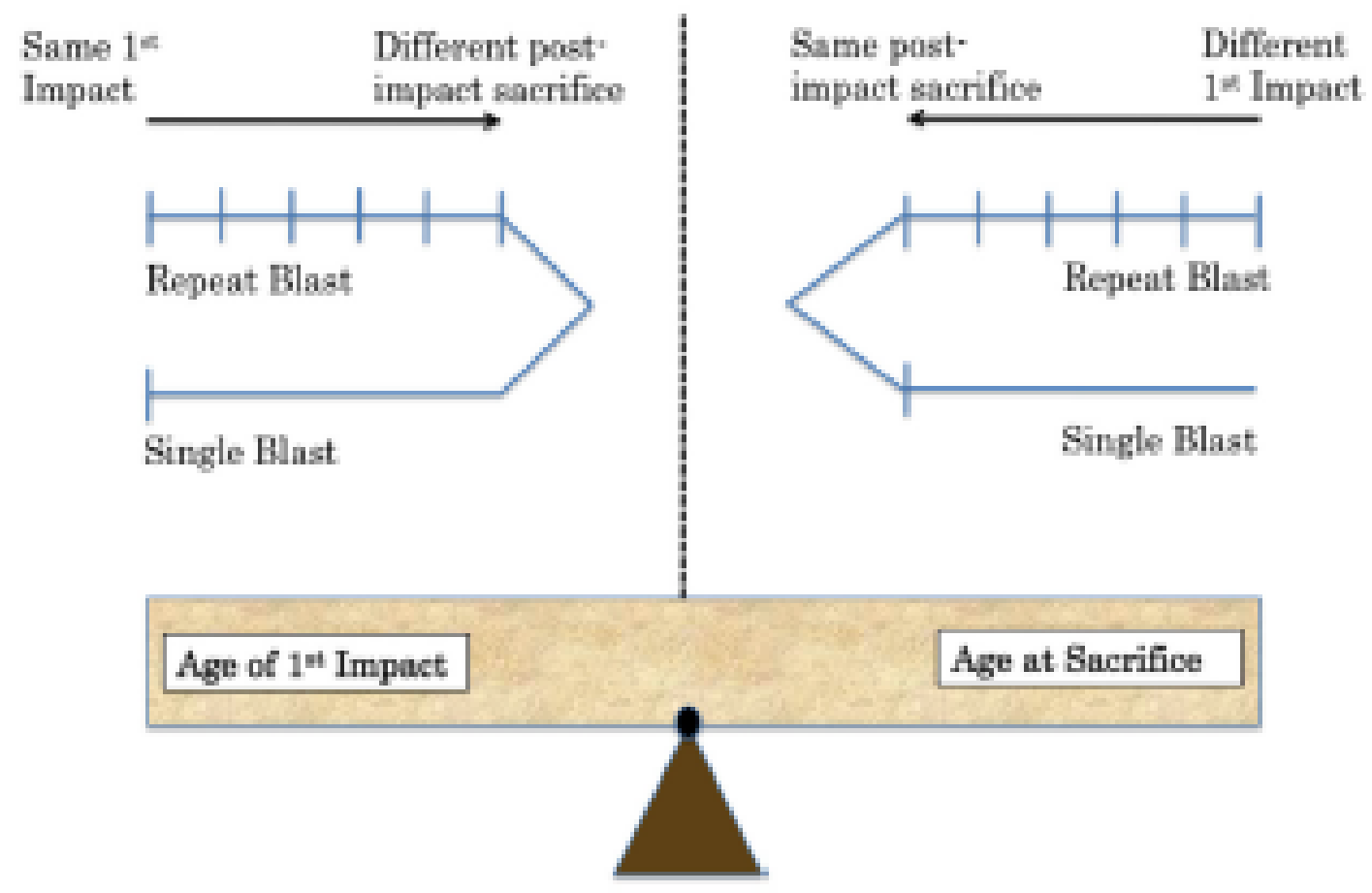

Figure 7.2. Methodological challenges associated with repeat injury in comparison to single injury paradigms include balancing equal age at time of exposure versus age at sacrifice. Two possibilities are shown depending on the variable the experimenter wants to control in future work. 
Table 7.1. Chronic traumatic encephalopathy TBI models

\begin{tabular}{|c|c|c|c|c|c|c|}
\hline Study & Sex/species/age & Model & Injuries & Interval & Anesthesia & Outcome measures \\
\hline $\begin{array}{l}\text { Liu } \\
\text { et al. (27) }\end{array}$ & $\begin{array}{l}\text { Male } \\
\text { S. Dawley rat } \\
2-3 \text { months }\end{array}$ & $\begin{array}{l}\text { Metal CCl } \\
\text { Open head }\end{array}$ & Single & Single & Isoflurane & Tauopathy; cell death; apoptosis \\
\hline $\begin{array}{l}\text { Goldstein } \\
\text { et al. (17) }\end{array}$ & $\begin{array}{l}\text { Male } \\
\text { C57BL6 mice } \\
2-3 \text { months }\end{array}$ & $\begin{array}{l}\text { Blast } \\
\text { Closed head }\end{array}$ & Single & Single & $\begin{array}{l}\text { Ketamine/ } \\
\text { xylazine }\end{array}$ & $\begin{array}{l}\text { Electrophysiology; tauopathy; axonal damage; } \\
\text { motor; cognition; structural integrity; advanced } \\
\text { imaging; human studies }\end{array}$ \\
\hline $\begin{array}{l}\text { Ojo et al. } \\
(18)\end{array}$ & $\begin{array}{l}\text { Male/female } \\
\text { C57BL6 mice, hTau Tg mice } \\
18 \text { months }\end{array}$ & $\begin{array}{l}\text { Metal CCl } \\
\text { Closed head }\end{array}$ & $\begin{array}{l}\text { Single } \\
\text { Repeat (5) }\end{array}$ & Single (48 h) & Isoflurane & $\begin{array}{l}\text { Tauopathy; gliosis and degeneration; structural } \\
\text { integrity; cell death }\end{array}$ \\
\hline $\begin{array}{l}\text { Mouzon } \\
\text { et al. (25) }\end{array}$ & $\begin{array}{l}\text { Male } \\
\text { C57BL6 mice } \\
9-15 \text { months }\end{array}$ & $\begin{array}{l}\text { Metal } \mathrm{CCl} \\
\text { Closed head }\end{array}$ & $\begin{array}{l}\text { Single } \\
\text { Repeat (5) }\end{array}$ & Single $(48 \mathrm{~h})$ & Isoflurane & $\begin{array}{l}\text { Motor; cognition; anxiety; inflammation; tauopathy; } \\
\text { axonal damage }\end{array}$ \\
\hline $\begin{array}{l}\text { Huber } \\
\text { et al. (26) }\end{array}$ & $\begin{array}{l}\text { Male } \\
\text { C57BL6 mice } \\
2-3 \text { months }\end{array}$ & $\begin{array}{l}\text { Blast } \\
\text { Closed head }\end{array}$ & Single & Single & Isoflurane & Motor; oxidative stress; tauopathy \\
\hline $\begin{array}{l}\text { Luo et al. } \\
\text { (22) }\end{array}$ & $\begin{array}{l}\text { Male } \\
\text { C57BL6 mice, } \\
\text { GFAP Luc mice } \\
2-3 \text { months }\end{array}$ & $\begin{array}{l}\text { Rubber } \mathrm{CCl} \\
\text { Closed head }\end{array}$ & $\begin{array}{l}\text { Single } \\
\text { Repeat } \\
(2,3,5)\end{array}$ & Single $(24 \mathrm{~h})$ & Isoflurane & $\begin{array}{l}\text { Bioluminescence; motor; anxiety; cognition; fear } \\
\text { conditioning; gliosis and degeneration; apoptosis }\end{array}$ \\
\hline $\begin{array}{l}\text { Glushakova } \\
\text { et al. (39) }\end{array}$ & $\begin{array}{l}\text { Male } \\
\text { S. Dawley rats } \\
2-3 \text { months }\end{array}$ & $\begin{array}{l}\text { Metal CCl } \\
\text { Open head }\end{array}$ & Single & Single & Isoflurane & $\begin{array}{l}\text { Vascular and axonal damage; gliosis and } \\
\text { degeneration }\end{array}$ \\
\hline $\begin{array}{l}\text { Zhang } \\
\text { et al. (32) }\end{array}$ & $\begin{array}{l}\text { Male } \\
\text { C57BL6 mice } \\
\text { 2-3 months }\end{array}$ & $\begin{array}{l}\text { Metal CCl } \\
\text { Closed head }\end{array}$ & $\begin{array}{l}\text { Single } \\
\text { Repeat (3) }\end{array}$ & Single (24 h) & Avertin & $\begin{array}{l}\text { Electrophysiology; neuroscore; inflammation; } \\
\text { tauopathy; gliosis and degeneration; cognition }\end{array}$ \\
\hline $\begin{array}{l}\text { Kondo } \\
\text { et al. (29) }\end{array}$ & $\begin{array}{l}\text { Male } \\
\text { C57BL6 mice } \\
\text { 2-3 months }\end{array}$ & $\begin{array}{l}\text { Single } \\
\text { blast and } \\
\text { weightdrop } \\
\text { Closed head }\end{array}$ & $\begin{array}{l}\text { Single } \\
\text { Repeat (7) }\end{array}$ & $\begin{array}{l}\text { Single severe } \\
\text { Seven mild } \\
\text { over } 9 \text { days }\end{array}$ & Isoflurane & $\begin{array}{l}\text { Electrophysiology; motor; cognition; anxiety; } \\
\text { structural integrity; axonal damage; tauopathy; cell } \\
\text { death; mitochondrial function; human studies }\end{array}$ \\
\hline $\begin{array}{l}\text { Lucke-Wold } \\
\text { et al. }(24 \text {, } \\
\text { 30) }\end{array}$ & $\begin{array}{l}\text { Male } \\
\text { S. Dawley rats } \\
2-3 \text { months }\end{array}$ & $\begin{array}{l}\text { Blast } \\
\text { Closed head }\end{array}$ & $\begin{array}{l}\text { Single } \\
\text { Repeat (6) }\end{array}$ & $\begin{array}{l}\text { Single } \\
\text { Six mild over } \\
10 \text { days }\end{array}$ & Isoflurane & $\begin{array}{l}\text { Cognition; endoplasmic reticulum stress; tauopathy; } \\
\text { human studies }\end{array}$ \\
\hline
\end{tabular}


Table 7.2. Shock tube TBI models

\begin{tabular}{|c|c|c|c|c|c|c|}
\hline Study & Sex/species/age & Model & Injuries & Interval & Anesthesia & Outcome measures \\
\hline $\begin{array}{l}\text { Long } \\
\text { et al. (74) }\end{array}$ & $\begin{array}{l}\text { Male } \\
\text { S. Dawley rats } \\
2-3 \text { months }\end{array}$ & $\begin{array}{l}\text { 5.3-m metal } \\
\text { tube }\end{array}$ & Single & Single & Isoflurane & $\begin{array}{l}\text { Cardiovascular; motor; cognition; structural integrity; } \\
\text { vascular damage; degeneration }\end{array}$ \\
\hline $\begin{array}{l}\text { Budde } \\
\text { et al. (75) }\end{array}$ & $\begin{array}{l}\text { Unknown } \\
\text { S. Dawley rats } \\
\text { Unknown }\end{array}$ & $\begin{array}{l}\text { 3.3-m metal } \\
\text { tube }\end{array}$ & $\begin{array}{l}\text { Single mild } \\
\text { and severe }\end{array}$ & Single & Isoflurane & $\begin{array}{l}\text { Advanced imaging; anxiety; cognition; gliosis and } \\
\text { degeneration; apoptosis }\end{array}$ \\
\hline $\begin{array}{l}\text { Genovese } \\
\text { et al. ( } 50 \text { ) }\end{array}$ & $\begin{array}{l}\text { Male } \\
\text { S. Dawley rats } \\
2-3 \text { months }\end{array}$ & $\begin{array}{l}\text { 5.3-m metal } \\
\text { tube }\end{array}$ & Repeat (3) & $24 \mathrm{~h}$ & Isoflurane & Fear conditioning \\
\hline $\begin{array}{l}\text { Wang et al. } \\
(52)\end{array}$ & $\begin{array}{l}\text { Male } \\
\text { C57BL6 mice } \\
\text { 2-3 months }\end{array}$ & $\begin{array}{l}\text { 5.3-m metal } \\
\text { tube }\end{array}$ & Repeat (3) & 1 or $30 \mathrm{~min}$ & Isoflurane & $\begin{array}{l}\text { Mitochondrial function; DNA fragmentation; righting } \\
\text { reflex; apoptosis }\end{array}$ \\
\hline $\begin{array}{l}\text { Lucke-Wold } \\
\text { et al. }(6,42)\end{array}$ & $\begin{array}{l}\text { Male } \\
\text { S. Dawley rats } \\
2-3 \text { months }\end{array}$ & $\begin{array}{l}0.3-\mathrm{m} \text { metal } \\
\text { tube }\end{array}$ & Single & Single & Isoflurane & $\begin{array}{l}\text { Vascular damage; structural integrity; gliosis and } \\
\text { degeneration }\end{array}$ \\
\hline $\begin{array}{l}\text { Logsdon et al. } \\
\text { (41) }\end{array}$ & $\begin{array}{l}\text { Male } \\
\text { S. Dawley rats } \\
2-3 \text { months }\end{array}$ & $\begin{array}{l}0.3-m \text { metal } \\
\text { tube }\end{array}$ & Single & Single & Isoflurane & $\begin{array}{l}\text { Vascular damage; endoplasmic reticulum stress; cell } \\
\text { death; apoptosis; anxiety }\end{array}$ \\
\hline
\end{tabular}


Table 7.3. Weight-drop TBI models

\begin{tabular}{|c|c|c|c|c|c|c|}
\hline Study & Sex/species/age & Model & Injuries & Interval & Anesthesia & Outcome measures \\
\hline $\begin{array}{l}\text { DeFord } \\
\text { et al. (72) }\end{array}$ & $\begin{array}{l}\text { Male } \\
\text { C57BL6 mice } \\
\text { 2-3 months }\end{array}$ & $\begin{array}{l}\text { Weight-drop } \\
\text { Closed head }\end{array}$ & $\begin{array}{l}\text { Single } \\
\text { Repeat (4) }\end{array}$ & Single $(24 \mathrm{~h})$ & $\begin{array}{l}\text { Isoflurane } \\
\mathrm{N}_{2} \mathrm{O} \text { and } \mathrm{O}_{2}(70: 30)\end{array}$ & $\begin{array}{l}\text { Neuroscore; cell death; vascular } \\
\text { damage; cardiovascular; cognition }\end{array}$ \\
\hline $\begin{array}{l}\text { Creeley } \\
\text { et al. }(48)\end{array}$ & $\begin{array}{l}\text { Male } \\
\text { C57BL6 mice } \\
2-3 \text { months }\end{array}$ & $\begin{array}{l}\text { Weight-drop } \\
\text { Closed head }\end{array}$ & Repeat (3) & $24 \mathrm{~h}$ & Isoflurane & $\begin{array}{l}\text { Motor; cognition; righting reflex; } \\
\text { cell death }\end{array}$ \\
\hline $\begin{array}{l}\text { Fujita } \\
\text { et al. (53) }\end{array}$ & $\begin{array}{l}\text { Male } \\
\text { S. Dawley rats } \\
3-6 \text { months }\end{array}$ & $\begin{array}{l}\text { Weight-drop } \\
\text { Skull-exposed }\end{array}$ & $\begin{array}{l}\text { Single } \\
\text { Repeat mild }(2,3) \\
\text { Repeat medium (2) } \\
\text { Repeat severe (2) }\end{array}$ & $\begin{array}{l}\text { Single } \\
\text { Two mild over } 3 \mathrm{~h} \\
\text { Three mild over } 2 \mathrm{~h} \\
\text { Two medium over } 3 \mathrm{~h} \\
\text { Two severe over } 3 \mathrm{~h} \\
\text { Two severe over } 5 \mathrm{~h} \\
\text { Two severe over } 10 \mathrm{~h}\end{array}$ & Pentobarbital & $\begin{array}{l}\text { Vascular reactivity to } \mathrm{ACh} \text {; axonal } \\
\text { damage }\end{array}$ \\
\hline $\begin{array}{l}\text { Meehan } \\
\text { et al. (54) }\end{array}$ & $\begin{array}{l}\text { Male } \\
\text { C57BL6 mice } \\
2-3 \text { months }\end{array}$ & $\begin{array}{l}\text { Weight-drop } \\
\text { Closed head }\end{array}$ & $\begin{array}{l}\text { Single } \\
\text { Repeat daily }(3,10) \\
\text { Repeat variable }(5)\end{array}$ & $\begin{array}{l}\text { Single } \\
\text { Daily }(3,5 \text {, or } 10) \\
\text { Weekly }(5) \\
\text { Monthly (5) }\end{array}$ & $\begin{array}{l}\text { Isoflurane } \\
\mathrm{N}_{2} \mathrm{O} \text { and } \mathrm{O}_{2}(70: 30)\end{array}$ & $\begin{array}{l}\text { Edema; axonal and vascular } \\
\text { damage; cell death; cognition }\end{array}$ \\
\hline $\begin{array}{l}\text { Mannix } \\
\text { et al. (54) }\end{array}$ & $\begin{array}{l}\text { Male } \\
\text { C57BL6 mice } \\
\text { 2-3 months }\end{array}$ & $\begin{array}{l}\text { Weight-drop } \\
\text { Closed head }\end{array}$ & $\begin{array}{l}\text { Single } \\
\text { Repeat daily }(5,7) \\
\text { Repeat variable }(5)\end{array}$ & $\begin{array}{l}\text { Single } \\
\text { Daily (5 or } 7 \text { ) } \\
\text { Weekly (5) } \\
\text { Biweekly (5) } \\
\text { Monthly (5) }\end{array}$ & $\begin{array}{l}\text { Isoflurane } \\
\mathrm{N}_{2} \mathrm{O} \text { and } \mathrm{O}_{2}(70: 30)\end{array}$ & $\begin{array}{l}\text { Cognition; tauopathy; advanced } \\
\text { imaging }\end{array}$ \\
\hline $\begin{array}{l}\text { Weil } \\
\text { et al. (57) }\end{array}$ & $\begin{array}{l}\text { Male } \\
\text { Swiss web. mice } \\
2-3 \text { months }\end{array}$ & $\begin{array}{l}\text { Weight-drop } \\
\text { Skull-exposed }\end{array}$ & $\begin{array}{l}\text { Single } \\
\text { Repeat (2) }\end{array}$ & Single 3 or 20 days & Isoflurane & $\begin{array}{l}\text { Glucose metabolism; } \\
\text { inflammation; gliosis and } \\
\text { degeneration; cell death; cognition }\end{array}$ \\
\hline
\end{tabular}


Table 7.4. TBI models not using craniotomy

\begin{tabular}{|c|c|c|c|c|c|c|}
\hline Study & Sex/species/age & Model & Injuries & Interval & Anesthesia & Outcome measures \\
\hline $\begin{array}{l}\text { Mouzon } \\
\text { et al. (65) }\end{array}$ & $\begin{array}{l}\text { Male } \\
\text { C57BL6 mice } \\
\text { 2-3 months }\end{array}$ & $\begin{array}{l}\text { Metal CCl } \\
\text { Closed head }\end{array}$ & $\begin{array}{l}\text { Single } \\
\text { Repeat (5) }\end{array}$ & Single $(48 h)$ & Isoflurane & $\begin{array}{l}\text { Motor; cognition; gliosis and degeneration; righting } \\
\text { reflex; axonal damage }\end{array}$ \\
\hline $\begin{array}{l}\text { Yoshiyama } \\
\text { et al. (35) }\end{array}$ & $\begin{array}{l}\text { Male/Female } \\
\text { B6D2/F1 mice, } \\
\text { Tau Tg mice } \\
12 \text { months }\end{array}$ & $\begin{array}{l}\text { Silicone } \mathrm{CCl} \\
\text { Skull-exposed }\end{array}$ & Repeat (16) & $\begin{array}{l}\text { Four per day } \\
\text { Every } 20 \text { min } \\
\text { Once a week for } \\
4 \text { weeks }\end{array}$ & Isoflurane & $\begin{array}{l}\text { Neuroscore; cognition; gliosis and degeneration; } \\
\text { tauopathy }\end{array}$ \\
\hline $\begin{array}{l}\text { Laurer } \\
\text { et al. (64) }\end{array}$ & $\begin{array}{l}\text { Male } \\
\text { C57BL6 mice } \\
2-3 \text { months }\end{array}$ & $\begin{array}{l}\text { Rubber CCl } \\
\text { Skull-exposed }\end{array}$ & $\begin{array}{l}\text { Single } \\
\text { Repeat (2) }\end{array}$ & Single $(24 \mathrm{~h})$ & Pentobarbital & $\begin{array}{l}\text { Neuroscore; motor; cardiovascular; cognition; } \\
\text { axonal and vascular damage; cell death; tauopathy }\end{array}$ \\
\hline $\begin{array}{l}\text { Bolton and } \\
\text { Saatman (56) }\end{array}$ & $\begin{array}{l}\text { Male } \\
\text { C57BL6 mice } \\
2-3 \text { months }\end{array}$ & $\begin{array}{l}\text { Silicone } \mathrm{CCl} \\
\text { Skull-exposed }\end{array}$ & $\begin{array}{l}\text { Single } \\
\text { Repeat (5) }\end{array}$ & Single ( 24 or $48 \mathrm{~h}$ ) & Isoflurane & $\begin{array}{l}\text { Cardiovascular; righting reflex; axonal damage; } \\
\text { gliosis and degeneration; tauopathy }\end{array}$ \\
\hline $\begin{array}{l}\text { Shitaka } \\
\text { et al. (49) }\end{array}$ & $\begin{array}{l}\text { Male } \\
\text { C57BL6 mice } \\
2-3 \text { months }\end{array}$ & $\begin{array}{l}\text { Rubber } \mathrm{CCl} \\
\text { Skull-exposed }\end{array}$ & Repeat (2) & $24 \mathrm{~h}$ & Isoflurane & $\begin{array}{l}\text { Cognition; structural integrity; gliosis and } \\
\text { degeneration; axonal damage; electron microscopy }\end{array}$ \\
\hline $\begin{array}{l}\text { Klemenhagen } \\
\text { et al. (51) }\end{array}$ & $\begin{array}{l}\text { Male } \\
\text { C57BL6 mice } \\
2-3 \text { months }\end{array}$ & $\begin{array}{l}\text { Rubber } \mathrm{CCl} \\
\text { Skull-exposed }\end{array}$ & Repeat (2) & $24 \mathrm{~h}$ & Isoflurane & $\begin{array}{l}\text { Fear conditioning; cognition; social recognition; } \\
\text { depression; anhedonia; gliosis; vascular damage }\end{array}$ \\
\hline $\begin{array}{l}\text { Uryu } \\
\text { et al. (33) }\end{array}$ & $\begin{array}{l}\text { Male/Female } \\
\text { B6D2/F1 mice, } \\
\text { APP Tg mice } \\
9-12 \text { months }\end{array}$ & $\begin{array}{l}\text { Rubber CCl } \\
\text { Skull-exposed }\end{array}$ & $\begin{array}{l}\text { Single } \\
\text { Repeat (2) }\end{array}$ & Single (24 h) & Pentobarbital & $\begin{array}{l}\text { Neuroscore; cognition; motor; vascular damage; } \\
\text { gliosis and degeneration; tauopathy; oxidative } \\
\text { stress }\end{array}$ \\
\hline $\begin{array}{l}\text { Longhi } \\
\text { et al. ( } 47)\end{array}$ & $\begin{array}{l}\text { Male } \\
\text { C57BL6 mice } \\
\text { 2-3 months }\end{array}$ & $\begin{array}{l}\text { Silicone } \mathrm{CCl} \\
\text { Skull-exposed }\end{array}$ & $\begin{array}{l}\text { Single } \\
\text { Repeat (2) }\end{array}$ & $\begin{array}{l}\text { Single }(3,5 \text {, or } \\
7 \text { days })\end{array}$ & Isoflurane & $\begin{array}{l}\text { Cognition; motor; righting reflex; gliosis and } \\
\text { degeneration; axonal and cytoskeletal damage; cell } \\
\text { death; edema }\end{array}$ \\
\hline $\begin{array}{l}\text { Conte } \\
\text { et al. (34) }\end{array}$ & $\begin{array}{l}\text { Female } \\
\text { B6D2/F1 mice, APP } \\
\text { Tg mice } \\
9-12 \text { months }\end{array}$ & $\begin{array}{l}\text { Rubber } \mathrm{CCl} \\
\text { Skull-exposed }\end{array}$ & Repeat (2) & $24 \mathrm{~h}$ & Isoflurane & $\begin{array}{l}\text { Cognition; tauopathy; structural integrity; oxidative } \\
\text { stress }\end{array}$ \\
\hline
\end{tabular}


Table 7.5. TBI models using craniotomy

\begin{tabular}{|c|c|c|c|c|c|c|}
\hline Study & Sex/species/age & Model & Injuries & Interval & Anesthesia & Outcome measures \\
\hline $\begin{array}{l}\text { Olsson } \\
\text { et al. (68) }\end{array}$ & $\begin{array}{l}\text { Rabbits } \\
\text { Unknown }\end{array}$ & Fluid percussion & $\begin{array}{l}\text { Single } \\
\text { Repeat (10) }\end{array}$ & Single (5 min) & Pentobarbital & $\begin{array}{l}\text { Righting reflex; cardiovascular; } \\
\text { vascular damage }\end{array}$ \\
\hline $\begin{array}{l}\text { Smith } \\
\text { et al. (40) }\end{array}$ & $\begin{array}{l}\text { Male } \\
\text { C57BL6 mice } \\
2-3 \text { months }\end{array}$ & $\begin{array}{l}\text { Metal CCl } \\
\text { Open head }\end{array}$ & Single & Single & Pentobarbital & $\begin{array}{l}\text { Cognition; structural integrity; cell } \\
\text { death; gliosis and degeneration; } \\
\text { vascular damage }\end{array}$ \\
\hline $\begin{array}{l}\text { Kanayama } \\
\text { et al. (31) }\end{array}$ & $\begin{array}{l}\text { Male } \\
\text { Wister rats } \\
2-3 \text { months }\end{array}$ & Fluid percussion & $\begin{array}{l}\text { Single } \\
\text { Repeat (7) }\end{array}$ & Single $(24 \mathrm{~h})$ & Pentobarbital & $\begin{array}{l}\text { Motor; social recognition; cytoskeletal } \\
\text { damage; tauopathy }\end{array}$ \\
\hline $\begin{array}{l}\text { Allen } \\
\text { et al. (158) }\end{array}$ & $\begin{array}{l}\text { Male } \\
\text { S. Dawley rats } \\
2-3 \text { months }\end{array}$ & $\begin{array}{l}\text { Weight-drop } \\
\text { Plexiglas piston }\end{array}$ & $\begin{array}{l}\text { Single severe } \\
\text { Repeat mild (3) }\end{array}$ & $\begin{array}{l}\text { Three mild over } \\
14 \text { days } \pm \text { severe } \\
3 \text { days }\end{array}$ & $\begin{array}{l}\text { Pentobarbital } \\
\text { or ketamine/ } \\
\text { rhompamine }\end{array}$ & $\begin{array}{l}\text { Motor; gliosis and degeneration; } \\
\text { structural integrity }\end{array}$ \\
\hline $\begin{array}{l}\text { DeRoss } \\
\text { et al. ( } 70)\end{array}$ & $\begin{array}{l}\text { Male } \\
\text { Long-Evans rats } \\
2-3 \text { months }\end{array}$ & Fluid percussion & $\begin{array}{l}\text { Single } \\
\text { Repeat }(2,3)\end{array}$ & Single (N/A) & Isoflurane & Cognition; motor \\
\hline $\begin{array}{l}\text { Manley } \\
\text { et al. (73) }\end{array}$ & $\begin{array}{l}\text { Male } \\
\text { Yorkshire pigs } \\
\text { Adult }\end{array}$ & $\begin{array}{l}\text { Metal CCl } \\
\text { Open head }\end{array}$ & Single & Single & $\begin{array}{l}\text { Pancuronium } \\
\text { Isoflurane }\end{array}$ & $\begin{array}{l}\text { Cardiovascular; structural integrity; } \\
\text { edema; vascular damage; cell death; } \\
\text { gliosis and degeneration }\end{array}$ \\
\hline $\begin{array}{l}\text { Donovan } \\
\text { et al. (61) }\end{array}$ & $\begin{array}{l}\text { Male } \\
\text { S. Dawley rats } \\
2-3 \text { months }\end{array}$ & $\begin{array}{l}\text { Metal } \mathrm{CCl} \\
\text { Open head }\end{array}$ & $\begin{array}{l}\text { Single } \\
\text { Repeat (2) }\end{array}$ & $\begin{array}{l}\text { Single } 7 \text { days } \\
\text { each side }\end{array}$ & Isoflurane & $\begin{array}{l}\text { Advanced imaging; structural } \\
\text { integrity; axonal damage }\end{array}$ \\
\hline $\begin{array}{l}\text { Hawkins } \\
\text { et al. (61) }\end{array}$ & $\begin{array}{l}\text { Male } \\
\text { S. Dawley Rats } \\
6-8 \text { months }\end{array}$ & Fluid percussion & Single & Single & Isoflurane & Extensive tauopathy assessment \\
\hline $\begin{array}{l}\text { Rubenstein } \\
\text { et al. (38) }\end{array}$ & $\begin{array}{l}\text { Male } \\
\text { S. Dawley rats and } \\
\text { C57BL6 mice } \\
2-3 \text { months }\end{array}$ & $\begin{array}{l}\text { Metal } \mathrm{CCl} \\
\text { Open head }\end{array}$ & Single & Single & Isoflurane & $\begin{array}{l}\text { Extensive tauopathy assessment; } \\
\text { human studies }\end{array}$ \\
\hline $\begin{array}{l}\text { Begum } \\
\text { et al. (37) }\end{array}$ & $\begin{array}{l}\text { Male } \\
\text { S. Dawley rats } \\
2-3 \text { months }\end{array}$ & $\begin{array}{l}\text { Metal CCl } \\
\text { Open head }\end{array}$ & Single & Single & Isoflurane & $\begin{array}{l}\text { Motor; endoplasmic reticulum stress; } \\
\text { tauopathy; axonal damage }\end{array}$ \\
\hline $\begin{array}{l}\text { Aungst } \\
\text { et al. (67) }\end{array}$ & $\begin{array}{l}\text { Male } \\
\text { S. Dawley rats } \\
2-3 \text { months }\end{array}$ & Fluid percussion & $\begin{array}{l}\text { Single } \\
\text { Repeat (3) }\end{array}$ & Single $(48 \mathrm{~h})$ & Isoflurane & $\begin{array}{l}\text { Electrophysiology; neuroscore; } \\
\text { cognition; social recognition; gliosis } \\
\text { and degeneration; cell death }\end{array}$ \\
\hline
\end{tabular}




\section{Table 7.6. Other in vivo TBI models}

\begin{tabular}{|c|c|c|c|c|c|c|}
\hline Study & Sex/species/age & Model & Injuries & Interval & Anesthesia & Outcome measures \\
\hline $\begin{array}{l}\text { Raghupathi } \\
\text { et al. (69) }\end{array}$ & $\begin{array}{l}\text { Male } \\
\text { Farm pigs } \\
3-5 \text { days }\end{array}$ & $\begin{array}{l}\text { Non-impact } \\
\text { head rotation }\end{array}$ & $\begin{array}{l}\text { Single } \\
\text { Repeat }\end{array}$ & Single (15 min) & Isoflurane & $\begin{array}{l}\text { Cardiovascular; axonal and vascular damage; } \\
\text { structural integrity; cell death }\end{array}$ \\
\hline Friess et al. (58) & $\begin{array}{l}\text { Male } \\
\text { Farm pigs } \\
3-5 \text { days }\end{array}$ & $\begin{array}{l}\text { Non-impact } \\
\text { head rotation }\end{array}$ & $\begin{array}{l}\text { Single } \\
\text { Repeat (2) }\end{array}$ & $\begin{array}{l}\text { Single }(24 \mathrm{~h} \text { or } \\
7 \text { days }\end{array}$ & Isoflurane & Motor; cognition; axonal damage \\
\hline Roth et al. (77) & $\begin{array}{l}\text { Male } \\
\text { S. Dawley rats and } \\
\text { C57BL6 mice } \\
2-3 \text { months }\end{array}$ & $\begin{array}{l}\text { Skull thinning } \\
\text { compression } \\
\text { Skull-exposed }\end{array}$ & Single & Single & $\begin{array}{l}\text { Ketamine/xylazine/ } \\
\text { acepromazine }\end{array}$ & $\begin{array}{l}\text { Inflammation; gliosis and degeneration; oxidative } \\
\text { stress; vascular damage; structural integrity; } \\
\text { advanced imaging; human studies }\end{array}$ \\
\hline $\begin{array}{l}\text { Petraglia et al. } \\
(20,23)\end{array}$ & $\begin{array}{l}\text { Male } \\
\text { C57BL6 mice } \\
\text { 2-3 months }\end{array}$ & $\begin{array}{l}\text { Rubber } \mathrm{CCl} \\
\text { Helmet }\end{array}$ & $\begin{array}{l}\text { Single } \\
\text { Repeat (42) }\end{array}$ & $\begin{array}{l}\text { Six per day every } \\
2 \text { h over } 7 \text { days }\end{array}$ & No Anesthesia & $\begin{array}{l}\text { Neuroscore; motor; cognition; anxiety; depression; } \\
\text { sleep }\end{array}$ \\
\hline
\end{tabular}


Table 7.7. In vitro and ex vivo TBI models

\begin{tabular}{|c|c|c|c|c|c|c|}
\hline Study & Model & Cell Line & Injuries & Interval & Severity & Outcome measures \\
\hline $\begin{array}{l}\text { Zander } \\
\text { et al. (43) }\end{array}$ & Primary blast & PC12 neurons & $\begin{array}{l}\text { Single } \\
\text { Repeat (3) }\end{array}$ & $\begin{array}{l}\text { Single } \\
\text { Three over } \\
20 \mathrm{~min}\end{array}$ & $\begin{array}{l}\text { Mild to } \\
\text { moderate }\end{array}$ & $\begin{array}{l}\text { Membrane permeability; cell viability; } \\
\text { axonal damage }\end{array}$ \\
\hline $\begin{array}{l}\text { LaPlaca and } \\
\text { Thibault (78) }\end{array}$ & Shear stress & Neuronal culture & Single & Single & Mild & $\begin{array}{l}\text { Membrane permeability; calcium influx; } \\
\text { cell death }\end{array}$ \\
\hline Morrison et al. (81) & Membrane strain & $\begin{array}{l}\text { Organo-typic } \\
\text { hippocampal slices }\end{array}$ & Single & Single & $\begin{array}{l}\text { Mild to } \\
\text { Severe }\end{array}$ & $\begin{array}{l}\text { Cell death; apoptosis; membrane } \\
\text { permeability }\end{array}$ \\
\hline Mukhin et al. (79) & Blade transection & Neuron/glial culture & Single & Single & Severe & Cell death; excitotoxicity \\
\hline Sieg et al. (80) & $\begin{array}{l}\text { Mechanical } \\
\text { compression }\end{array}$ & $\begin{array}{l}\text { Organo-typic cortical } \\
\text { slices }\end{array}$ & Single & Single & Severe & Cell death; apoptosis; axonal damage \\
\hline Slemmer et al. (63) & Cell stretch & Neuronal culture & $\begin{array}{l}\text { Single } \\
\text { Repeat (6) }\end{array}$ & $\begin{array}{l}\text { Single } \\
\text { Six over } 24 \mathrm{~h}\end{array}$ & Mild & Cell viability; cell death \\
\hline
\end{tabular}




\section{Chapter Eight}

\section{GENERAL DISCUSSION}




\section{SUMMARY}

A tremendous need for traumatic brain injury (TBI) therapy is driving the focus of research.

With over 250,000 TBI hospitalizations per year, the neuropsychiatric symptoms and cognitive deficits that develop after neurotrauma are detrimental to patients and even those around them. An estimated 60 billion dollars in annual healthcare costs are spent to treat the behavioral sequelae of TBI. This staggering cost warrants a desperate need for an effective treatment for TBI. Much like Stroke, pre-clinical therapeutic success has not translated into the clinic. Over the past few decades, the lack of clinical relevance in pre-clinical TBI models has prevented effective treatment options from enduring the rigor of clinical trials.

Historically, our lab has focused on making neural injury models more clinicallyrelevant. For instance, our aged model of embolic stroke was better recapitulating clinical ischemic stroke in a more relevant pre-clinical sub-population. We wanted to do the same for the sub-population of patients who sustain non-penetrating head trauma. As such, we developed a clinically-relevant model of blast-induced TBI for young Sprague-dawley rats. To develop our model we needed to understand the physical characteristics behind a blast wave and how to scale human exposure to rodent exposure. We scaled our model for rat exposure based on lung leathality curves and could gauge blast exposure from mild to severe. We could produce gross macroscopic hemorrage and mortality with the severe blast exposure, and microscopic neural injury and no mortality using the mild blast exposure. Because $75 \%$ of all TBI hospitalizations are mild in nature, and because mild TBI is often undiagnosed, we used the mild blast exposure for the rest of our experiments in order to encompass a larger clinical population and to explore underlying mechanism of symptom onset. 
A relatively new disease has been classified in patients in particular who have sustained head injuries and has been called Chronic Traumatic Encephalopathy (CTE). CTE is commonly reported in soldiers and athletes due to their susceptibility to multiple head injuries during their careers. Impulsivity and memory loss are two symptoms common amongst patients diagnosed with CTE. The cause of the disease is largely unknown and warrants further examination. New advanced imaging modalities have allowed clinicians to gain a better understanding of disease progression, but the pathophysiology of CTE is still in its infancy.

After model validation, we sought to characterize the temporal and regional profile of the blast injury. In addition, we wanted to evaluate rodent behaviors associated with the symptoms observed in patients with CTE. Furthermore, we investigated the neurophysiological response in brain slice recordings at various time points after TBI. Once we could determine where, when and to what extent, neural injury occurred, then we could correlate our biochemical and physiological findings to the behavioral changes associated with CTE. After blast injury, we discovered microscopic pathology which suggested damage to the neurovascular unit (NVU) in the hippocampus and cortex. We also revealed a sustained increase in biochemical markers of cell death such as DNA fragmentation and apoptosis after blast. A subtle deficit in short term plasticity was also observed at more chronic time points after TBI, and suggested a decreased probability of neurotransmitter release. Spatial memory deficits were also oberved using the Morris water maze at chronic time points after blast injury. Interestingly, we also observed an increase in the phosphorylation of Tau protein, which has been heavily linked to CTE. These results were merely a characterization, and more concrete follow-up studies were necessary to elucidate potential mechanisms that may lead to tau phosphorylation and subsequent CTE development. 
Tau is a microtubule stabilizing protein found in axons. When neural injury occurs, tau can become dissociated from axonal microtubules and become insoluble. Insoluble tau exposes multiple phosphorylation sites that are targets of many tau kinases. When tau becomes hyperphosphorylated, it changes its conformation and can bind to and aggregate with additional tau proteins to form an oligomer. Oligomeric tau is toxic to neurons and is thought to contribute to neurodegenerative diseases such as CTE. Therefore, we wanted to first determine the extent of tauopathy occurring in rodents exposed to our TBI model.

CTE is a progressive neurodegenerative disease and has been labeled as a tauopathy due to the extent of tau protein aggregation observed in post-mortem samples of those with the disease. Tau phosphorylation and aggregation are considered hallmarks of CTE development. We observed an increase in marker of tau phosphorylation and conformational change in rats exposed to single and repetitive blast. We also revealed that rats subject to repetitive blast injury displayed behavioral deficits associated with the neuropsychiatric symptoms observed in patients with CTE. Interestingly, Tau changes and cell death markers were discovered in brain regions that correlated to the observed behavioral deficits.

CTE, like many other neurodegenerative diseases have some vascular component to their pathophysiology. CTE shows a perivascular tau distribution in clinical samples, so we wanted to evaluate damage to the NVU in rodents. We observed an acute increase in Evan's blue extravasation in the frontal cortex and the hippocampus. We also observed astrocyte and microglia activation in the same regions, which suggested damage to the NVU. The link between damage to the NVU and neurobehavioral dysfunction following TBI is poorly understood. Interestingly, we also observed tau changes in brain regions where the damage to the NVU was revealed. We believe the primary effects of blast injury can result from damage to the NVU can 
trigger secondary injury cascades, which if go untreated, can eventually lead to neuronal cell death.

The overarching goal of this study was to elucidate a link between damage to the NVU and the development of tauopathies and cell death. This link would provide us with novel targets for pharmaceutical intervention to ameliorate the behavioral sequelae of CTE. Recently secondary injury cascades, such as endoplasmic reticulum (ER) stress and neuroinflammation, have been hypothesized to be early indicators for the development of tauopathy. We revealed a robust increase in markers of ER stress and neuroinflammation within the frontal cortex after TBI. Interestingly, we observed impulsive-like behavior in rats after TBI, which is indicative of damage to the frontal cortex. These findings support the literature and provides a new perspective to the link between vascular injury and neurodegeneration.

After characterization of secondary injury cascades, we investigated the role of ER stress modulation in mediating neuroinflammation, cell death and neurobehavioral dysfunction following TBI. ER stress modulation attenuated markers of neuroinflammation and neuronal cell death. Moreover, ER stress modulation ameliorated impulsive-like behavior and improved working memory in rats after TBI. These results suggest that the ER stress response may be the missing link between primary injury and tauopathy development. Elucidation of these mechanisms will provide new therapeutic options to reduce neuronal cell death after injury and hopefully reduce the symptoms of neurodegenerative diseases, such as CTE.

\section{FUTURE DIRECTIONS}

Because we can't predict, or prevent a TBI from occurring, and because it is difficult to treat neurodegenerative disease once they have been diagnosed, our only option to this point is to 
target and treat secondary injury cascades. Future studies should aim to causally link secondary injury cascades to tauopathy in order to elucidate new drug targets for improving TBI outcome. In order to implement an effective treatment strategy we need to intervene early after injury to avoid neurodegeneration and inevitable cell death. Therapeutic strategy could be biphasic in nature and contain an early intervention of ER stress modulation, followed by a prolonged suppression of neuroinflammation for optimal results. Developing effective treatment for TBI may be more than just finding novel targets, but also determining the rights times to intervene.

Better modeling has allowed for more extensive biochemical and behavioral characteristics to be defined. Now that our laboratory has established a potential CTE model, options for translational investigation of CTE pathophysiology become available. We have revealed damage to the NVU, tau pathology, and neurobehavioral dysfunction in rats exposed to our TBI model. Bridging the gap between these effects remains the key to therapeutic success. Therefore, we investigated secondary injury cascades, such as ER stress and neuroinflammation, as potential CTE mechanisms for novel therapeutic intervention. By improving our understanding about CTE mechanisms, significant strides can be made to develop novel therapeutic strategies to reduce the neuropsychiatric burdens of patients exposed to neurotrauma. 


\section{REFERENCES}

Abdul-Muneer PM, Chandra N, Haorah J (2014) Interactions of Oxidative Stress and Neurovascular Inflammation in the Pathogenesis of Traumatic Brain Injury. Mol Neurobiol.

Abdul-Muneer PM, Schuetz H, Wang F, Skotak M, Jones J, Gorantla S, Zimmerman MC, Chandra N, Haorah J (2013) Induction of oxidative and nitrosative damage leads to cerebrovascular inflammation in an animal model of mild traumatic brain injury induced by primary blast. Free Radic Biol Med 60:282-291.

Abdullah Z, Bayraktutan U (2014) NADPH oxidase mediates TNF-alpha-evoked in vitro brain barrier dysfunction: roles of apoptosis and time. Mol Cell Neurosci 61:72-84.

Abisambra JF, Scheff S (2014) Brain injury in the context of tauopathies. J Alzheimers Dis 40:495-518.

Adalbert R, Nogradi A, Babetto E, Janeckova L, Walker SA, Kerschensteiner M, Misgeld T, Coleman MP (2009) Severely dystrophic axons at amyloid plaques remain continuous and connected to viable cell bodies. Brain 132:402-416.

Adhikari A, Topiwala MA, Gordon JA (2011) Single units in the medial prefrontal cortex with anxiety-related firing patterns are preferentially influenced by ventral hippocampal activity. Neuron 71:898-910.

Adibhatla RM, Hatcher JF (2010) Lipid oxidation and peroxidation in CNS health and disease: from molecular mechanisms to therapeutic opportunities. Antioxid Redox Signal 12:125169.

Ahlers ST, Vasserman-Stokes E, Shaughness MC, Hall AA, Shear DA, Chavko M, McCarron RM, Stone JR (2012) Assessment of the effects of acute and repeated exposure to blast overpressure in rodents: toward a greater understanding of blast and the potential ramifications for injury in humans exposed to blast. Front Neurol 3:32.

Albert-Weissenberger C, Siren AL (2010) Experimental traumatic brain injury. Exp Transl Stroke Med 2:16.

Alford PW, Dabiri BE, Goss JA, Hemphill MA, Brigham MD, Parker KK (2011) Blast-induced phenotypic switching in cerebral vasospasm. Proc Natl Acad Sci U S A 108:1270512710.

Algattas H, Huang JH (2014) Traumatic Brain Injury pathophysiology and treatments: early, intermediate, and late phases post-injury. Int J Mol Sci 15:309-341.

Allen GV, Gerami D, Esser MJ (2000) Conditioning effects of repetitive mild neurotrauma on motor function in an animal model of focal brain injury. Neuroscience 99:93-105.

Almli LM, Stevens JS, Smith AK, Kilaru V, Meng Q, Flory J, Abu-Amara D, Hammamieh R, Yang R, Mercer KB, Binder EB, Bradley B, Hamilton S, Jett M, Yehuda R, Marmar CR, Ressler KJ (2015) A genome-wide identified risk variant for PTSD is a methylation quantitative trait locus and confers decreased cortical activation to fearful faces. Am J Med Genet B Neuropsychiatr Genet 168B:327-336.

Alves JL (2014) Blood-brain barrier and traumatic brain injury. J Neurosci Res 92:141-147.

Amantini A, Carrai R, Lori S, Peris A, Amadori A, Pinto F, Grippo A (2012) Neurophysiological monitoring in adult and pediatric intensive care. Minerva Anestesiol 78:1067-1075.

Anderson J, Sandhir R, Hamilton ES, Berman NE (2009) Impaired expression of neuroprotective molecules in the HIF-1alpha pathway following traumatic brain injury in aged mice. $\mathrm{J}$ Neurotrauma 26:1557-1566. 
Andres AL, Regev L, Phi L, Seese RR, Chen Y, Gall CM, Baram TZ (2013) NMDA receptor activation and calpain contribute to disruption of dendritic spines by the stress neuropeptide CRH. J Neurosci 33:16945-16960.

Angelo M, Plattner F, Giese KP (2006) Cyclin-dependent kinase 5 in synaptic plasticity, learning and memory. J Neurochem 99:353-370.

Angoa-Perez M, Kane MJ, Briggs DI, Herrera-Mundo N, Viano DC, Kuhn DM (2014) Animal models of sports-related head injury: bridging the gap between pre-clinical research and clinical reality. J Neurochem 129:916-931.

Ansari MA, Roberts KN, Scheff SW (2014) A time course of NADPH-oxidase up-regulation and endothelial nitric oxide synthase activation in the hippocampus following neurotrauma. Free Radic Biol Med 77:21-29.

Arun P, Abu-Taleb R, Oguntayo S, Tanaka M, Wang Y, Valiyaveettil M, Long JB, Zhang Y, Nambiar MP (2013) Distinct patterns of expression of traumatic brain injury biomarkers after blast exposure: role of compromised cell membrane integrity. Neurosci Lett 552:8791.

Asakuno K, Ishida A (2014) Intraarterial vasodilator therapy immediately rescued pure cortical deafness due to bilateral cerebral vasospasm. Surg Neurol Int 5:61.

Asl SZ, Khaksari M, Khachki AS, Shahrokhi N, Nourizade S (2013) Contribution of estrogen receptors alpha and beta in the brain response to traumatic brain injury. $\mathrm{J}$ Neurosurg 119:353-361.

Aungst SL, Kabadi SV, Thompson SM, Stoica BA, Faden AI (2014) Repeated mild traumatic brain injury causes chronic neuroinflammation, changes in hippocampal synaptic plasticity, and associated cognitive deficits. J Cereb Blood Flow Metab 34:1223-1232.

Baalman K, Cotton J, Rasband N, Rasband M (2012) Blast Wave Exposure Impairs Memory and Decreases Axon Initial Segment Length. J Neurotrauma.

Baalman KL, Cotton RJ, Rasband SN, Rasband MN (2013) Blast wave exposure impairs memory and decreases axon initial segment length. J Neurotrauma 30:741-751.

Bach-y-Rita P (2003) Theoretical basis for brain plasticity after a TBI. Brain Inj 17:643-651.

Badaut J, Bix GJ (2014) Vascular neural network phenotypic transformation after traumatic injury: potential role in long-term sequelae. Transl Stroke Res 5:394-406.

Badiola N, Penas C, Minano-Molina A, Barneda-Zahonero B, Fado R, Sanchez-Opazo G, Comella JX, Sabria J, Zhu C, Blomgren K, Casas C, Rodriguez-Alvarez J (2011) Induction of ER stress in response to oxygen-glucose deprivation of cortical cultures involves the activation of the PERK and IRE-1 pathways and of caspase-12. Cell Death Dis 2:e149.

Bailes JE, Patel V (2014) The potential for DHA to mitigate mild traumatic brain injury. Mil Med 179:112-116.

Bailes JE, Dashnaw ML, Petraglia AL, Turner RC (2014) Cumulative effects of repetitive mild traumatic brain injury. Prog Neurol Surg 28:50-62.

Bailes JE, Petraglia AL, Omalu BI, Nauman E, Talavage T (2013) Role of subconcussion in repetitive mild traumatic brain injury. J Neurosurg 119:1235-1245.

Bailey ZS, Sujith Sajja VS, Hubbard WB, VandeVord PJ (2015a) Blast Induced Neurotrauma Leads To Changes In The Epigenome. Biomed Sci Instrum 51:423-430.

Bailey ZS, Grinter MB, De La Torre Campos D, VandeVord PJ (2015b) Blast induced neurotrauma causes overpressure dependent changes to the DNA methylation equilibrium. Neurosci Lett 604:119-123. 
Balestreri M, Czosnyka M, Chatfield DA, Steiner LA, Schmidt EA, Smielewski P, Matta B, Pickard JD (2004) Predictive value of Glasgow Coma Scale after brain trauma: change in trend over the past ten years. J Neurol Neurosurg Psychiatry 75:161-162.

Banks SJ, Mayer B, Obuchowski N, Shin W, Lowe M, Phillips M, Modic M, Bernick C (2014) Impulsiveness in professional fighters. J Neuropsychiatry Clin Neurosci 26:44-50.

Barnes DE, Kaup A, Kirby KA, Byers AL, Diaz-Arrastia R, Yaffe K (2014) Traumatic brain injury and risk of dementia in older veterans. Neurology 83:312-319.

Bass CR, Panzer MB, Rafaels KA, Wood G, Shridharani J, Capehart B (2012) Brain injuries from blast. Ann Biomed Eng 40:185-202.

Bauer AM, Rasmussen PA (2014) Treatment of intracranial vasospasm following subarachnoid hemorrhage. Front Neurol 5:72.

Baugh CM, Robbins CA, Stern RA, McKee AC (2014) Current understanding of chronic traumatic encephalopathy. Curr Treat Options Neurol 16:306.

Baugh CM, Stamm JM, Riley DO, Gavett BE, Shenton ME, Lin A, Nowinski CJ, Cantu RC, McKee AC, Stern RA (2012) Chronic traumatic encephalopathy: neurodegeneration following repetitive concussive and subconcussive brain trauma. Brain Imaging Behav 6:244-254.

Beerten J, Schymkowitz J, Rousseau F (2012) Aggregation prone regions and gatekeeping residues in protein sequences. Curr Top Med Chem 12:2470-2478.

Begum G, Harvey L, Dixon CE, Sun D (2013) ER stress and effects of DHA as an ER stress inhibitor. Transl Stroke Res 4:635-642.

Begum G, Yan HQ, Li L, Singh A, Dixon CE, Sun D (2014) Docosahexaenoic Acid Reduces ER Stress and Abnormal Protein Accumulation and Improves Neuronal Function Following Traumatic Brain Injury. J Neurosci 34:3743-3755.

Bell KR, Pepping M (2001) Women and traumatic brain injury. Phys Med Rehabil Clin N Am 12:169-182.

Bellezza I, Grottelli S, Mierla AL, Cacciatore I, Fornasari E, Roscini L, Cardinali G, Minelli A (2014) Neuroinflammation and endoplasmic reticulum stress are coregulated by cyclo(His-Pro) to prevent LPS neurotoxicity. Int J Biochem Cell Biol 51:159-169.

Bernales S, McDonald KL, Walter P (2006) Autophagy counterbalances endoplasmic reticulum expansion during the unfolded protein response. PLoS Biol 4:e423.

Bernales S, Soto MM, McCullagh E (2012) Unfolded protein stress in the endoplasmic reticulum and mitochondria: a role in neurodegeneration. Front Aging Neurosci 4:5.

Biancardi VC, Son SJ, Ahmadi S, Filosa JA, Stern JE (2014) Circulating angiotensin II gains access to the hypothalamus and brain stem during hypertension via breakdown of the blood-brain barrier. Hypertension 63:572-579.

Bidzan L, Bidzan M, Pachalska M (2012) Aggressive and impulsive behavior in Alzheimer's disease and progression of dementia. Med Sci Monit 18:CR182-189.

Blaylock RL, Maroon J (2011) Immunoexcitotoxicity as a central mechanism in chronic traumatic encephalopathy-A unifying hypothesis. Surg Neurol Int 2:107.

Blennow K, Hardy J, Zetterberg H (2012) The neuropathology and neurobiology of traumatic brain injury. Neuron 76:886-899.

Bolton AN, Saatman KE (2014) Regional neurodegeneration and gliosis are amplified by mild traumatic brain injury repeated at 24-hour intervals. J Neuropathol Exp Neurol 73:933947. 
Bor-Seng-Shu E, Figueiredo EG, Fonoff ET, Fujimoto Y, Panerai RB, Teixeira MJ (2013)

Decompressive craniectomy and head injury: brain morphometry, ICP, cerebral hemodynamics, cerebral microvascular reactivity, and neurochemistry. Neurosurg Rev 36:361-370.

Bowen IG, Fletcher ER, Richmond DR, Hirsch FG, White CS (1968) Biophysical mechanisms and scaling procedures applicable in assessing responses of the thorax energized by airblast overpressures or by nonpenetrating missiles. Ann N Y Acad Sci 152:122-146.

Bower JH, Maraganore DM, Peterson BJ, McDonnell SK, Ahlskog JE, Rocca WA (2003) Head trauma preceding PD: a case-control study. Neurology 60:1610-1615.

Boyce M, Bryant KF, Jousse C, Long K, Harding HP, Scheuner D, Kaufman RJ, Ma D, Coen DM, Ron D, Yuan J (2005) A selective inhibitor of eIF2alpha dephosphorylation protects cells from ER stress. Science 307:935-939.

Bracchi-Ricard V, Lambertsen KL, Ricard J, Nathanson L, Karmally S, Johnstone J, Ellman DG, Frydel B, McTigue DM, Bethea JR (2013) Inhibition of astroglial NF-kappaB enhances oligodendrogenesis following spinal cord injury. J Neuroinflammation 10:92.

Bramlett HM, Dietrich WD (2001) Neuropathological protection after traumatic brain injury in intact female rats versus males or ovariectomized females. J Neurotrauma 18:891-900.

Brandner S, Kellermann I, Hore N, Bozhkov Y, Buchfelder M (2014) Clinical Course Score (CCS): A New Clinical Score to Evaluate Efficacy of Neurotrauma Treatment in Traumatic Brain Injury and Subarachnoid Hemorrhage. J Neurosurg Anesthesiol.

Brennan AM, Suh SW, Won SJ, Narasimhan P, Kauppinen TM, Lee H, Edling Y, Chan PH, Swanson RA (2009) NADPH oxidase is the primary source of superoxide induced by NMDA receptor activation. Nat Neurosci 12:857-863.

Brody DL, Benetatos J, Bennett RE, Klemenhagen KC, Mac Donald CL (2015) The pathophysiology of repetitive concussive traumatic brain injury in experimental models; new developments and open questions. Mol Cell Neurosci.

Brown CM, Suzuki S, Jelks KA, Wise PM (2009) Estradiol is a potent protective, restorative, and trophic factor after brain injury. Semin Reprod Med 27:240-249.

Bruce-Keller AJ, Dimayuga FO, Reed JL, Wang C, Angers R, Wilson ME, Dimayuga VM, Scheff SW (2007) Gender and estrogen manipulation do not affect traumatic brain injury in mice. J Neurotrauma 24:203-215.

Bruns J, Jr., Hauser WA (2003) The epidemiology of traumatic brain injury: a review. Epilepsia 44 Suppl 10:2-10.

Budde MD, Shah A, McCrea M, Cullinan WE, Pintar FA, Stemper BD (2013) Primary blast traumatic brain injury in the rat: relating diffusion tensor imaging and behavior. Front Neurol 4:154.

Bukovics P, Czeiter E, Amrein K, Kovacs N, Pal J, Tamas A, Bagoly T, Helyes Z, Buki A, Reglodi D (2014) Changes of PACAP level in cerebrospinal fluid and plasma of patients with severe traumatic brain injury. Peptides.

Bullock R, Zauner A, Woodward JJ, Myseros J, Choi SC, Ward JD, Marmarou A, Young HF (1998) Factors affecting excitatory amino acid release following severe human head injury. J Neurosurg 89:507-518.

Carlson SW, Madathil SK, Sama DM, Gao X, Chen J, Saatman KE (2014) Conditional overexpression of insulin-like growth factor-1 enhances hippocampal neurogenesis and restores immature neuron dendritic processes after traumatic brain injury. J Neuropathol Exp Neurol 73:734-746. 
Cernak I (2015) Blast Injuries and Blast-Induced Neurotrauma: Overview of Pathophysiology and Experimental Knowledge Models and Findings. In: Brain Neurotrauma: Molecular, Neuropsychological, and Rehabilitation Aspects (Kobeissy FHP, ed). Boca Raton (FL).

Cernak I, Noble-Haeusslein LJ (2010) Traumatic brain injury: an overview of pathobiology with emphasis on military populations. J Cereb Blood Flow Metab 30:255-266.

Cernak I, Wang Z, Jiang J, Bian X, Savic J (2001) Cognitive deficits following blast injuryinduced neurotrauma: possible involvement of nitric oxide. Brain Inj 15:593-612.

Cernak I, Merkle AC, Koliatsos VE, Bilik JM, Luong QT, Mahota TM, Xu L, Slack N, Windle D, Ahmed FA (2011) The pathobiology of blast injuries and blast-induced neurotrauma as identified using a new experimental model of injury in mice. Neurobiol Dis 41:538551.

Chalouhi N, Whiting A, Anderson EC, Witte S, Zanaty M, Tjoumakaris S, Gonzalez LF, Hasan D, Starke RM, Hann S, Ghobrial GM, Rosenwasser R, Jabbour P (2014) Comparison of techniques for ventriculoperitoneal shunting in 523 patients with subarachnoid hemorrhage. J Neurosurg:1-4.

Champion HR, Holcomb JB, Young LA (2009) Injuries from explosions: physics, biophysics, pathology, and required research focus. J Trauma 66:1468-1477; discussion 1477.

Chaudhari N, Talwar P, Parimisetty A, Lefebvre d'Hellencourt C, Ravanan P (2014) A molecular web: endoplasmic reticulum stress, inflammation, and oxidative stress. Front Cell Neurosci 8:213.

Chavko M, Watanabe T, Adeeb S, Lankasky J, Ahlers ST, McCarron RM (2011) Relationship between orientation to a blast and pressure wave propagation inside the rat brain. $\mathrm{J}$ Neurosci Methods 195:61-66.

Chen B, Mutschler M, Yuan Y, Neugebauer E, Huang Q, Maegele M (2013a) Superimposed traumatic brain injury modulates vasomotor responses in third-order vessels after hemorrhagic shock. Scand J Trauma Resusc Emerg Med 21:77.

Chen Y, Huang W, Constantini S (2013b) Concepts and strategies for clinical management of blast-induced traumatic brain injury and posttraumatic stress disorder. J Neuropsychiatry Clin Neurosci 25:103-110.

Chen Y, Huang W, Constantini S (2013c) The Differences between Blast-Induced and SportsRelated Brain Injuries. Front Neurol 4:119.

Cheng J, Gu J, Ma Y, Yang T, Kuang Y, Li B, Kang J (2010) Development of a rat model for studying blast-induced traumatic brain injury. J Neurol Sci 294:23-28.

Cho HJ, Sajja VS, Vandevord PJ, Lee YW (2013) Blast induces oxidative stress, inflammation, neuronal loss and subsequent short-term memory impairment in rats. Neuroscience 253:9-20.

Chodobski A, Zink BJ, Szmydynger-Chodobska J (2011) Blood-brain barrier pathophysiology in traumatic brain injury. Transl Stroke Res 2:492-516.

Clark RS, Chen J, Watkins SC, Kochanek PM, Chen M, Stetler RA, Loeffert JE, Graham SH (1997) Apoptosis-suppressor gene bcl-2 expression after traumatic brain injury in rats. J Neurosci 17:9172-9182.

Clark RS, Kochanek PM, Watkins SC, Chen M, Dixon CE, Seidberg NA, Melick J, Loeffert JE, Nathaniel PD, Jin KL, Graham SH (2000) Caspase-3 mediated neuronal death after traumatic brain injury in rats. J Neurochem 74:740-753.

Colley BS, Phillips LL, Reeves TM (2010) The effects of cyclosporin-A on axonal conduction deficits following traumatic brain injury in adult rats. Exp Neurol 224:241-251. 
Combes RD (2013) A critical review of anaesthetised animal models and alternatives for military research, testing and training, with a focus on blast damage, haemorrhage and resuscitation. Altern Lab Anim 41:385-415.

Conte V, Uryu K, Fujimoto S, Yao Y, Rokach J, Longhi L, Trojanowski JQ, Lee VM, McIntosh TK, Pratico D (2004) Vitamin E reduces amyloidosis and improves cognitive function in Tg2576 mice following repetitive concussive brain injury. J Neurochem 90:758-764.

Corcoran KA, Maren S (2001) Hippocampal inactivation disrupts contextual retrieval of fear memory after extinction. J Neurosci 21:1720-1726.

Corsellis JA, Bruton CJ, Freeman-Browne D (1973) The aftermath of boxing. Psychol Med 3:270-303.

Costa RO, Ferreiro E, Martins I, Santana I, Cardoso SM, Oliveira CR, Pereira CM (2012) Amyloid beta-induced ER stress is enhanced under mitochondrial dysfunction conditions. Neurobiol Aging 33:824 e825-816.

Coughlin JM, Wang Y, Munro CA, Ma S, Yue C, Chen S, Airan R, Kim PK, Adams AV, Garcia C, Higgs C, Sair HI, Sawa A, Smith G, Lyketsos CG, Caffo B, Kassiou M, Guilarte TR, Pomper MG (2014) Neuroinflammation and brain atrophy in former NFL players: An in vivo multimodal imaging pilot study. Neurobiol Dis 74C:58-65.

Covey DC, Born CT (2010) Blast injuries: mechanics and wounding patterns. J Surg Orthop Adv 19:8-12.

Creed JA, DiLeonardi AM, Fox DP, Tessler AR, Raghupathi R (2011) Concussive brain trauma in the mouse results in acute cognitive deficits and sustained impairment of axonal function. J Neurotrauma 28:547-563.

Creeley CE, Wozniak DF, Bayly PV, Olney JW, Lewis LM (2004) Multiple episodes of mild traumatic brain injury result in impaired cognitive performance in mice. Acad Emerg Med 11:809-819.

Cui T, Zhu G (2014) Ulinastatin Attenuates Brain Edema After Traumatic Brain Injury in Rats. Cell Biochem Biophys.

Cullen DK, Vernekar VN, LaPlaca MC (2011) Trauma-induced plasmalemma disruptions in three-dimensional neural cultures are dependent on strain modality and rate. $\mathbf{J}$ Neurotrauma 28:2219-2233.

Cullen NK, Crescini C, Bayley MT (2009) Rehabilitation outcomes after anoxic brain injury: a case-controlled comparison with traumatic brain injury. PM R 1:1069-1076.

Dagher JH, Habra N, Lamoureux J, De Guise E, Feyz M (2010) Global outcome in acute phase of treatment following moderate-to-severe traumatic brain injury from motor vehicle collisions vs assaults. Brain Inj 24:1389-1398.

Dalle Lucca JJ, Chavko M, Dubick MA, Adeeb S, Falabella MJ, Slack JL, McCarron R, Li Y (2012) Blast-induced moderate neurotrauma (BINT) elicits early complement activation and tumor necrosis factor alpha (TNFalpha) release in a rat brain. J Neurol Sci 318:146154.

Dapul HR, Park J, Zhang J, Lee C, DanEshmand A, Lok J, Ayata C, Gray T, Scalzo A, Qiu J, Lo EH, Whalen MJ (2013) Concussive injury before or after controlled cortical impact exacerbates histopathology and functional outcome in a mixed traumatic brain injury model in mice. J Neurotrauma 30:382-391.

Dash PK, Hylin MJ, Hood KN, Orsi SA, Zhao J, Redell JB, Tsvetkov AS, Moore AN (2015) Inhibition of eIF2alpha phosphatase reduces tissue damage and improves learning and memory following experimental traumatic brain injury. J Neurotrauma. 
Day NL, Floyd CL, D'Alessandro TL, Hubbard WJ, Chaudry IH (2013) 17beta-estradiol confers protection after traumatic brain injury in the rat and involves activation of $\mathrm{G}$ proteincoupled estrogen receptor 1. J Neurotrauma 30:1531-1541.

De Beaumont L, Henry LC, Gosselin N (2012) Long-term functional alterations in sports concussion. Neurosurg Focus 33:E8: 1-7.

de Lanerolle NC, Bandak F, Kang D, Li AY, Du F, Swauger P, Parks S, Ling G, Kim JH (2011) Characteristics of an explosive blast-induced brain injury in an experimental model. $\mathbf{J}$ Neuropathol Exp Neurol 70:1046-1057.

DeFord SM, Wilson MS, Rice AC, Clausen T, Rice LK, Barabnova A, Bullock R, Hamm RJ (2002) Repeated mild brain injuries result in cognitive impairment in B6C3F1 mice. J Neurotrauma 19:427-438.

Deniaud A, Sharaf el dein O, Maillier E, Poncet D, Kroemer G, Lemaire C, Brenner C (2008) Endoplasmic reticulum stress induces calcium-dependent permeability transition, mitochondrial outer membrane permeabilization and apoptosis. Oncogene 27:285-299.

Dennis AM, Haselkorn ML, Vagni VA, Garman RH, Janesko-Feldman K, Bayir H, Clark RS, Jenkins LW, Dixon CE, Kochanek PM (2009) Hemorrhagic shock after experimental traumatic brain injury in mice: effect on neuronal death. J Neurotrauma 26:889-899.

Depreitere B, Guiza F, Van den Berghe G, Schuhmann MU, Maier G, Piper I, Meyfroidt G (2014) Pressure autoregulation monitoring and cerebral perfusion pressure target recommendation in patients with severe traumatic brain injury based on minute-byminute monitoring data. J Neurosurg 120:1451-1457.

DeRoss AL, Adams JE, Vane DW, Russell SJ, Terella AM, Wald SL (2002) Multiple head injuries in rats: effects on behavior. J Trauma 52:708-714.

Deslauriers AM, Afkhami-Goli A, Paul AM, Bhat RK, Acharjee S, Ellestad KK, Noorbakhsh F, Michalak M, Power C (2011) Neuroinflammation and endoplasmic reticulum stress are coregulated by crocin to prevent demyelination and neurodegeneration. J Immunol 187:4788-4799.

DeWitt DS, Prough DS (2009) Blast-induced brain injury and posttraumatic hypotension and hypoxemia. J Neurotrauma 26:877-887.

DiLeonardi AM, Huh JW, Raghupathi R (2009) Impaired axonal transport and neurofilament compaction occur in separate populations of injured axons following diffuse brain injury in the immature rat. Brain Res 1263:174-182.

Dileonardi AM, Huh JW, Raghupathi R (2012) Differential effects of FK506 on structural and functional axonal deficits after diffuse brain injury in the immature rat. J Neuropathol Exp Neurol 71:959-972.

Dobrowolska JA, Gibson CJ (2005) Immunohistochemical analysis of amyloid precursor protein in the hippocampus and cortex of mice following reepated mild traumatic brain injuries. In: Society for Neuroscience.

Donovan V, Bianchi A, Hartman R, Bhanu B, Carson MJ, Obenaus A (2012) Computational analysis reveals increased blood deposition following repeated mild traumatic brain injury. Neuroimage Clin 1:18-28.

Donovan V, Kim C, Anugerah AK, Coats JS, Oyoyo U, Pardo AC, Obenaus A (2014) Repeated mild traumatic brain injury results in long-term white-matter disruption. J Cereb Blood Flow Metab 34:715-723. 
Dore-Duffy P, Wang S, Mehedi A, Katyshev V, Cleary K, Tapper A, Reynolds C, Ding Y, Zhan P, Rafols J, Kreipke CW (2011) Pericyte-mediated vasoconstriction underlies TBIinduced hypoperfusion. Neurol Res 33:176-186.

Dubrovsky AS, Friedman D, Kocilowicz H (2014) Pediatric post-traumatic headaches and peripheral nerve blocks of the scalp: a case series and patient satisfaction survey. Headache 54:878-887.

Duckworth JL, Grimes J, Ling GS (2012) Pathophysiology of battlefield associated traumatic brain injury. Pathophysiology.

Elder GA, Cristian A (2009) Blast-related mild traumatic brain injury: mechanisms of injury and impact on clinical care. Mt Sinai J Med 76:111-118.

Elder GA, Dorr NP, De Gasperi R, Gama Sosa MA, Shaughness MC, Maudlin-Jeronimo E, Hall AA, McCarron RM, Ahlers ST (2012) Blast exposure induces post-traumatic stress disorder-related traits in a rat model of mild traumatic brain injury. J Neurotrauma 29:2564-2575.

Engel DC, Mies G, Terpolilli NA, Trabold R, Loch A, De Zeeuw CI, Weber JT, Maas AI, Plesnila N (2008) Changes of cerebral blood flow during the secondary expansion of a cortical contusion assessed by 14C-iodoantipyrine autoradiography in mice using a noninvasive protocol. J Neurotrauma 25:739-753.

Ewing-Cobbs L, Prasad M, Kramer L, Louis PT, Baumgartner J, Fletcher JM, Alpert B (2000) Acute neuroradiologic findings in young children with inflicted or noninflicted traumatic brain injury. Childs Nerv Syst 16:25-33; discussion 34.

Faden AI, Demediuk P, Panter SS, Vink R (1989) The role of excitatory amino acids and NMDA receptors in traumatic brain injury. Science 244:798-800.

Faden AI, Wu J, Stoica BA, Loane DJ (2015) Progressive inflammatory-mediated neurodegeneration after traumatic brain or spinal cord injury. Br J Pharmacol.

Farace E, Alves WM (2000a) Do women fare worse? A metaanalysis of gender differences in outcome after traumatic brain injury. Neurosurg Focus 8:e6.

Farace E, Alves WM (2000b) Do women fare worse: a metaanalysis of gender differences in traumatic brain injury outcome. J Neurosurg 93:539-545.

Farook JM, Shields J, Tawfik A, Markand S, Sen T, Smith SB, Brann D, Dhandapani KM, Sen N (2013) GADD34 induces cell death through inactivation of Akt following traumatic brain injury. Cell Death Dis 4:e754.

Farrell-Carnahan L, Franke L, Graham C, McNamee S (2013) Subjective sleep disturbance in veterans receiving care in the Veterans Affairs Polytrauma System following blast-related mild traumatic brain injury. Mil Med 178:951-956.

Faust K, Horn P, Schneider UC, Vajkoczy P (2014) Blood pressure changes after aneurysmal subarachnoid hemorrhage and their relationship to cerebral vasospasm and clinical outcome. Clin Neurol Neurosurg 125C:36-40.

Fenn AM, Skendelas JP, Moussa DN, Muccigrosso MM, Popovich PG, Lifshitz J, Eiferman DS, Godbout JP (2015) Methylene blue attenuates traumatic brain injury-associated neuroinflammation and acute depressive-like behavior in mice. J Neurotrauma 32:127138.

Ferguson S, Mouzon B, Kayihan G, Wood M, Poon F, Doore S, Mathura V, Humphrey J, O'Steen B, Hayes R, Roses A, Mullan M, Crawford F (2010) Apolipoprotein E genotype and oxidative stress response to traumatic brain injury. Neuroscience 168:811-819. 
Finnie JW (2013) Neuroinflammation: beneficial and detrimental effects after traumatic brain injury. Inflammopharmacology 21:309-320.

Fischer AH, Jacobson KA, Rose J, Zeller R (2008) Hematoxylin and eosin staining of tissue and cell sections. CSH Protoc 2008:pdb prot4986.

Foreman BP, Caesar RR, Parks J, Madden C, Gentilello LM, Shafi S, Carlile MC, Harper CR, Diaz-Arrastia RR (2007) Usefulness of the abbreviated injury score and the injury severity score in comparison to the Glasgow Coma Scale in predicting outcome after traumatic brain injury. J Trauma 62:946-950.

Foster KA, Recker MJ, Lee PS, Bell MJ, Tyler-Kabara EC (2014) Factors Associated with Hemispheric Hypodensity after Subdural Hematoma following Abusive Head Trauma in Children. J Neurotrauma.

Friess SH, Ichord RN, Ralston J, Ryall K, Helfaer MA, Smith C, Margulies SS (2009) Repeated traumatic brain injury affects composite cognitive function in piglets. J Neurotrauma 26:1111-1121.

Fujita M, Wei EP, Povlishock JT (2012a) Effects of hypothermia on cerebral autoregulatory vascular responses in two rodent models of traumatic brain injury. J Neurotrauma 29:1491-1498.

Fujita M, Wei EP, Povlishock JT (2012b) Intensity- and interval-specific repetitive traumatic brain injury can evoke both axonal and microvascular damage. J Neurotrauma 29:21722180.

Galehdar Z, Swan P, Fuerth B, Callaghan SM, Park DS, Cregan SP (2010) Neuronal apoptosis induced by endoplasmic reticulum stress is regulated by ATF4-CHOP-mediated induction of the Bcl-2 homology 3-only member PUMA. J Neurosci 30:16938-16948.

Gandy S, DeKosky ST (2014) [18F]-T807 tauopathy PET imaging in chronic traumatic encephalopathy. F1000Res 3:229.

Gao B, Zhang XY, Han R, Zhang TT, Chen C, Qin ZH, Sheng R (2013) The endoplasmic reticulum stress inhibitor salubrinal inhibits the activation of autophagy and neuroprotection induced by brain ischemic preconditioning. Acta Pharmacol Sin 34:657666.

Garman RH, Jenkins LW, Switzer RC, 3rd, Bauman RA, Tong LC, Swauger PV, Parks SA, Ritzel DV, Dixon CE, Clark RS, Bayir H, Kagan V, Jackson EK, Kochanek PM (2011) Blast exposure in rats with body shielding is characterized primarily by diffuse axonal injury. J Neurotrauma 28:947-959.

Garringer JA, Niyonkuru C, McCullough EH, Loucks T, Dixon CE, Conley YP, Berga S, Wagner AK (2013) Impact of aromatase genetic variation on hormone levels and global outcome after severe TBI. J Neurotrauma 30:1415-1425.

Gatson JW, Liu MM, Abdelfattah K, Wigginton JG, Smith S, Wolf S, Simpkins JW, Minei JP (2012) Estrone is neuroprotective in rats after traumatic brain injury. J Neurotrauma 29:2209-2219.

Gavett BE, Cantu RC, Shenton M, Lin AP, Nowinski CJ, McKee AC, Stern RA (2011) Clinical appraisal of chronic traumatic encephalopathy: current perspectives and future directions. Curr Opin Neurol 24:525-531.

Genovese RF, Simmons LP, Ahlers ST, Maudlin-Jeronimo E, Dave JR, Boutte AM (2013) Effects of mild TBI from repeated blast overpressure on the expression and extinction of conditioned fear in rats. Neuroscience 254:120-129. 
Glushakova OY, Johnson D, Hayes RL (2014) Delayed Increases in Microvascular Pathology after Experimental Traumatic Brain Injury Are Associated with Prolonged Inflammation, Blood-Brain Barrier Disruption, and Progressive White Matter Damage. J Neurotrauma.

Goddeyne C, Nichols J, Wu C, Anderson T (2015) Repetitive mild traumatic brain injury induces ventriculomegaly and cortical thinning in juvenile rats. J Neurophysiol 113:32683280.

Goldstein LE, Fisher AM, Tagge CA, Zhang XL, Velisek L, Sullivan JA, Upreti C, Kracht JM, Ericsson M, Wojnarowicz MW, Goletiani CJ, Maglakelidze GM, Casey N, Moncaster JA, Minaeva O, Moir RD, Nowinski CJ, Stern RA, Cantu RC, Geiling J, Blusztajn JK, Wolozin BL, Ikezu T, Stein TD, Budson AE, Kowall NW, Chargin D, Sharon A, Saman S, Hall GF, Moss WC, Cleveland RO, Tanzi RE, Stanton PK, McKee AC (2012) Chronic traumatic encephalopathy in blast-exposed military veterans and a blast neurotrauma mouse model. Sci Transl Med 4:134ra160.

Green KN, Martinez-Coria H, Khashwji H, Hall EB, Yurko-Mauro KA, Ellis L, LaFerla FM (2007) Dietary docosahexaenoic acid and docosapentaenoic acid ameliorate amyloid-beta and tau pathology via a mechanism involving presenilin 1 levels. J Neurosci 27:43854395.

Greer JE, Hanell A, McGinn MJ, Povlishock JT (2013) Mild traumatic brain injury in the mouse induces axotomy primarily within the axon initial segment. Acta Neuropathol 126:59-74.

Griesbach GS, Gomez-Pinilla F, Hovda DA (2004) The upregulation of plasticity-related proteins following TBI is disrupted with acute voluntary exercise. Brain Res 1016:154162.

Gurkoff G, Shahlaie K, Lyeth B, Berman R (2013) Voltage-gated calcium channel antagonists and traumatic brain injury. Pharmaceuticals (Basel) 6:788-812.

Guskiewicz KM, Marshall SW, Bailes J, McCrea M, Cantu RC, Randolph C, Jordan BD (2005) Association between recurrent concussion and late-life cognitive impairment in retired professional football players. Neurosurgery 57:719-726; discussion 719-726.

Guskiewicz KM, McCrea M, Marshall SW, Cantu RC, Randolph C, Barr W, Onate JA, Kelly JP (2003) Cumulative effects associated with recurrent concussion in collegiate football players: the NCAA Concussion Study. JAMA 290:2549-2555.

Habets RL, Borst JG (2005) Post-tetanic potentiation in the rat calyx of Held synapse. J Physiol 564:173-187.

Hagberg H, Wilson MA, Matsushita H, Zhu C, Lange M, Gustavsson M, Poitras MF, Dawson TM, Dawson VL, Northington F, Johnston MV (2004) PARP-1 gene disruption in mice preferentially protects males from perinatal brain injury. J Neurochem 90:1068-1075.

Haghighi F, Ge Y, Chen S, Xin Y, Umali MU, De Gasperi R, Gama Sosa MA, Ahlers ST, Elder GA (2015) Neuronal DNA Methylation Profiling of Blast-related Traumatic Brain Injury (TBI). J Neurotrauma.

Hamm RJ, Jenkins LW, Lyeth BG, White-Gbadebo DM, Hayes RL (1991) The effect of age on outcome following traumatic brain injury in rats. J Neurosurg 75:916-921.

Hamm RJ, Dixon CE, Gbadebo DM, Singha AK, Jenkins LW, Lyeth BG, Hayes RL (1992) Cognitive deficits following traumatic brain injury produced by controlled cortical impact. J Neurotrauma 9:11-20.

Harmon KG, Drezner J, Gammons M, Guskiewicz K, Halstead M, Herring S, Kutcher J, Pana A, Putukian M, Roberts W, American Medical Society for Sports M (2013) American 
Medical Society for Sports Medicine position statement: concussion in sport. Clin J Sport Med 23:1-18.

Hartmann DA, Underly RG, Watson AN, Shih AY (2014) A murine toolbox for imaging the neurovascular unit. Microcirculation.

Hawkins BE, Krishnamurthy S, Castillo-Carranza DL, Sengupta U, Prough DS, Jackson GR, DeWitt DS, Kayed R (2013) Rapid accumulation of endogenous tau oligomers in a rat model of traumatic brain injury: possible link between traumatic brain injury and sporadic tauopathies. J Biol Chem 288:17042-17050.

Hayashi T (2015) Conversion of psychological stress into cellular stress response: roles of the sigma-1 receptor in the process. Psychiatry Clin Neurosci 69:179-191.

Helmy A, Carpenter KL, Menon DK, Pickard JD, Hutchinson PJ (2011a) The cytokine response to human traumatic brain injury: temporal profiles and evidence for cerebral parenchymal production. J Cereb Blood Flow Metab 31:658-670.

Helmy A, De Simoni MG, Guilfoyle MR, Carpenter KL, Hutchinson PJ (2011b) Cytokines and innate inflammation in the pathogenesis of human traumatic brain injury. Prog Neurobiol 95:352-372.

Hetz C, Chevet E, Harding HP (2013) Targeting the unfolded protein response in disease. Nat Rev Drug Discov 12:703-719.

Hillered L, Vespa PM, Hovda DA (2005) Translational neurochemical research in acute human brain injury: the current status and potential future for cerebral microdialysis. J Neurotrauma 22:3-41.

Hisanaga S, Endo R (2010) Regulation and role of cyclin-dependent kinase activity in neuronal survival and death. J Neurochem 115:1309-1321.

Ho KM, Honeybul S, Yip CB, Silbert BI (2014) Prognostic significance of blood-brain barrier disruption in patients with severe nonpenetrating traumatic brain injury requiring decompressive craniectomy. J Neurosurg:1-6.

Ho YS, Yang X, Yeung SC, Chiu K, Lau CF, Tsang AW, Mak JC, Chang RC (2012) Cigarette smoking accelerated brain aging and induced pre-Alzheimer-like neuropathology in rats. PLoS One 7:e36752.

Hoane MR, Lasley LA, Akstulewicz SL (2004) Middle age increases tissue vulnerability and impairs sensorimotor and cognitive recovery following traumatic brain injury in the rat. Behav Brain Res 153:189-197.

Hochstadter E, Stewart TC, Alharfi IM, Ranger A, Fraser DD (2014) Subarachnoid Hemorrhage Prevalence and Its Association with Short-Term Outcome in Pediatric Severe Traumatic Brain Injury. Neurocrit Care.

Hoozemans JJ, van Haastert ES, Eikelenboom P, de Vos RA, Rozemuller JM, Scheper W (2007) Activation of the unfolded protein response in Parkinson's disease. Biochem Biophys Res Commun 354:707-711.

Hu SL, Hu R, Li F, Liu Z, Xia YZ, Cui GY, Feng H (2008) Hyperbaric oxygen preconditioning protects against traumatic brain injury at high altitude. Acta Neurochir Suppl 105:191196.

Huang X, Chen Y, Zhang H, Ma Q, Zhang YW, Xu H (2012) Salubrinal attenuates betaamyloid-induced neuronal death and microglial activation by inhibition of the NFkappaB pathway. Neurobiol Aging 33:1007 e1009-1017.

Huber BR, Meabon JS, Martin TJ, Mourad PD, Bennett R, Kraemer BC, Cernak I, Petrie EC, Emery MJ, Swenson ER, Mayer C, Mehic E, Peskind ER, Cook DG (2013) Blast 
exposure causes early and persistent aberrant phospho- and cleaved-tau expression in a murine model of mild blast-induced traumatic brain injury. J Alzheimers Dis 37:309-323.

Huh JW, Widing AG, Raghupathi R (2008) Midline brain injury in the immature rat induces sustained cognitive deficits, bihemispheric axonal injury and neurodegeneration. Exp Neurol 213:84-92.

Huh JW, Franklin MA, Widing AG, Raghupathi R (2006) Regionally distinct patterns of calpain activation and traumatic axonal injury following contusive brain injury in immature rats. Dev Neurosci 28:466-476.

Hyde DW (2004) Conventional Weapons Effects Program. In. Vicksburg, MS: United States Army Corps of Engineers.

Iliff JJ, Chen MJ, Plog BA, Zeppenfeld DM, Soltero M, Yang L, Singh I, Deane R, Nedergaard M (2014) Impairment of glymphatic pathway function promotes tau pathology after traumatic brain injury. J Neurosci 34:16180-16193.

Inglese M, Bomsztyk E, Gonen O, Mannon LJ, Grossman RI, Rusinek H (2005) Dilated perivascular spaces: hallmarks of mild traumatic brain injury. AJNR Am J Neuroradiol 26:719-724.

Izzy S, Muehlschlegel S (2014) Cerebral vasospasm after aneurysmal subarachnoid hemorrhage and traumatic brain injury. Curr Treat Options Neurol 16:278.

Jalloh I, Carpenter KL, Grice P, Howe DJ, Mason A, Gallagher CN, Helmy A, Murphy MP, Menon DK, Carpenter TA, Pickard JD, Hutchinson PJ (2014) Glycolysis and the pentose phosphate pathway after human traumatic brain injury: microdialysis studies using 1,2-C glucose. J Cereb Blood Flow Metab.

Jayakumar AR, Tong XY, Ruiz-Cordero R, Bregy A, Bethea JR, Bramlett HM, Norenberg MD (2014) Activation of NF-kappaB mediates astrocyte swelling and brain edema in traumatic brain injury. J Neurotrauma 31:1249-1257.

Ji X, Tian Y, Xie K, Liu W, Qu Y, Fei Z (2012) Protective effects of hydrogen-rich saline in a rat model of traumatic brain injury via reducing oxidative stress. J Surg Res 178:e9-16.

Jia J, Guan D, Zhu W, Alkayed NJ, Wang MM, Hua Z, Xu Y (2009) Estrogen inhibits Fasmediated apoptosis in experimental stroke. Exp Neurol 215:48-52.

Jiang JY, Lyeth BG, Kapasi MZ, Jenkins LW, Povlishock JT (1992) Moderate hypothermia reduces blood-brain barrier disruption following traumatic brain injury in the rat. Acta Neuropathol 84:495-500.

Jilka SR, Scott G, Ham T, Pickering A, Bonnelle V, Braga RM, Leech R, Sharp DJ (2014) Damage to the Salience Network and interactions with the Default Mode Network. J Neurosci 34:10798-10807.

Johnson EM, Traver KL, Hoffman SW, Harrison CR, Herman JP (2013a) Environmental enrichment protects against functional deficits caused by traumatic brain injury. Front Behav Neurosci 7:44.

Johnson VE, Stewart W, Smith DH (2010) Traumatic brain injury and amyloid-beta pathology: a link to Alzheimer's disease? Nat Rev Neurosci 11:361-370.

Johnson VE, Stewart W, Smith DH (2013b) Axonal pathology in traumatic brain injury. Exp Neurol 246:35-43.

Johnson VE, Stewart JE, Begbie FD, Trojanowski JQ, Smith DH, Stewart W (2013c) Inflammation and white matter degeneration persist for years after a single traumatic brain injury. Brain 136:28-42. 
Kabadi SV, Faden AI (2014) Neuroprotective strategies for traumatic brain injury: improving clinical translation. Int J Mol Sci 15:1216-1236.

Kadyan V, Mysiw WJ, Bogner JA, Corrigan JD, Fugate LP, Clinchot DM (2004) Gender differences in agitation after traumatic brain injury. Am J Phys Med Rehabil 83:747-752.

Kamnaksh A, Kovesdi E, Kwon SK, Wingo D, Ahmed F, Grunberg NE, Long J, Agoston DV (2011) Factors affecting blast traumatic brain injury. J Neurotrauma 28:2145-2153.

Kamnaksh A, Kwon SK, Kovesdi E, Ahmed F, Barry ES, Grunberg NE, Long J, Agoston D (2012) Neurobehavioral, cellular, and molecular consequences of single and multiple mild blast exposure. Electrophoresis 33:3680-3692.

Kanayama G, Takeda M, Niigawa H, Ikura Y, Tamii H, Taniguchi N, Kudo T, Miyamae Y, Morihara T, Nishimura T (1996) The effects of repetitive mild brain injury on cytoskeletal protein and behavior. Methods Find Exp Clin Pharmacol 18:105-115.

Kane MJ, Angoa-Perez M, Francescutti DM, Sykes CE, Briggs DI, Leung LY, VandeVord PJ, Kuhn DM (2012) Altered gene expression in cultured microglia in response to simulated blast overpressure: possible role of pulse duration. Neurosci Lett 522:47-51.

Kasturi BS, Stein DG (2009) Progesterone decreases cortical and sub-cortical edema in young and aged ovariectomized rats with brain injury. Restor Neurol Neurosci 27:265-275.

Katayama Y, Becker DP, Tamura T, Hovda DA (1990) Massive increases in extracellular potassium and the indiscriminate release of glutamate following concussive brain injury. J Neurosurg 73:889-900.

Kaur C, Singh J, Lim MK, Ng BL, Yap EP, Ling EA (1995) The response of neurons and microglia to blast injury in the rat brain. Neuropathol Appl Neurobiol 21:369-377.

Kay A, Teasdale G (2001) Head injury in the United Kingdom. World J Surg 25:1210-1220.

Kaya M, Ahishali B (2011) Assessment of permeability in barrier type of endothelium in brain using tracers: Evans blue, sodium fluorescein, and horseradish peroxidase. Methods Mol Biol 763:369-382.

Ke C, Poon WS, Ng HK, Pang JC, Chan Y (2001) Heterogeneous responses of aquaporin-4 in oedema formation in a replicated severe traumatic brain injury model in rats. Neurosci Lett 301:21-24.

Kelman C, Reavey-Cantwell J (2014) Endovascular management of cerebral vasospasm. Neurosurg Focus 37:1.

Kessel D (2006) Protection of Bcl-2 by salubrinal. Biochem Biophys Res Commun 346:13201323.

Khan F, Baguley IJ, Cameron ID (2003) 4: Rehabilitation after traumatic brain injury. Med J Aust 178:290-295.

Kim H, Kim GD, Yoon BC, Kim K, Kim BJ, Choi Y, Czosnyka M, Oh BM, Kim DJ (2014) Quantitative analysis of computed tomography images and early detection of cerebral edema for pediatric traumatic brain injury patients: retrospective study. BMC Med $12: 186$.

Klemenhagen KC, O'Brien SP, Brody DL (2013) Repetitive concussive traumatic brain injury interacts with post-injury foot shock stress to worsen social and depression-like behavior in mice. PLoS One 8:e74510.

Kobeissy F, Mondello S, Tumer N, Toklu HZ, Whidden MA, Kirichenko N, Zhang Z, Prima V, Yassin W, Anagli J, Chandra N, Svetlov S, Wang KK (2013) Assessing neuro-systemic \& behavioral components in the pathophysiology of blast-related brain injury. Front Neurol 4:186. 
Kochanek PM, Bramlett H, Dietrich WD, Dixon CE, Hayes RL, Povlishock J, Tortella FC, Wang KK (2011) A novel multicenter preclinical drug screening and biomarker consortium for experimental traumatic brain injury: operation brain trauma therapy. $\mathbf{J}$ Trauma 71:S15-24.

Kochanek PM, Dixon CE, Shellington DK, Shin SS, Bayir H, Jackson EK, Kagan VE, Yan HQ, Swauger PV, Parks SA, Ritzel DV, Bauman R, Clark RS, Garman RH, Bandak F, Ling G, Jenkins LW (2013) Screening of biochemical and molecular mechanisms of secondary injury and repair in the brain after experimental blast-induced traumatic brain injury in rats. J Neurotrauma 30:920-937.

Koliatsos VE, Xu L (2015) The Problem of Neurodegeneration in Cumulative Sports Concussions: Emphasis on Neurofibrillary Tangle Formation. In: Brain Neurotrauma: Molecular, Neuropsychological, and Rehabilitation Aspects (Kobeissy FHP, ed). Boca Raton (FL).

Koliatsos VE, Cernak I, Xu L, Song Y, Savonenko A, Crain BJ, Eberhart CG, Frangakis CE, Melnikova T, Kim H, Lee D (2011) A mouse model of blast injury to brain: initial pathological, neuropathological, and behavioral characterization. J Neuropathol Exp Neurol 70:399-416.

Kondo A, Shahpasand K, Mannix R, Qiu J, Moncaster J, Chen CH, Yao Y, Lin YM, Driver JA, Sun Y, Wei S, Luo ML, Albayram O, Huang P, Rotenberg A, Ryo A, Goldstein LE, Pascual-Leone A, McKee AC, Meehan W, Zhou XZ, Lu KP (2015) Antibody against early driver of neurodegeneration cis P-tau blocks brain injury and tauopathy. Nature 523:431-436.

Koponen S, Taiminen T, Portin R, Himanen L, Isoniemi H, Heinonen H, Hinkka S, Tenovuo O (2002) Axis I and II psychiatric disorders after traumatic brain injury: a 30-year followup study. Am J Psychiatry 159:1315-1321.

Korczyn AD (2012) Why have we failed to cure Alzheimer's disease? J Alzheimers Dis 29:275282.

Kors EE, Terwindt GM, Vermeulen FL, Fitzsimons RB, Jardine PE, Heywood P, Love S, van den Maagdenberg AM, Haan J, Frants RR, Ferrari MD (2001) Delayed cerebral edema and fatal coma after minor head trauma: role of the CACNA1A calcium channel subunit gene and relationship with familial hemiplegic migraine. Ann Neurol 49:753-760.

Kovesdi E, Kamnaksh A, Wingo D, Ahmed F, Grunberg NE, Long JB, Kasper CE, Agoston DV (2012) Acute minocycline treatment mitigates the symptoms of mild blast-induced traumatic brain injury. Front Neurol 3:111.

Kramer AH, Le Roux P (2012) Red Blood Cell Transfusion and Transfusion Alternatives in Traumatic Brain Injury. Curr Treat Options Neurol.

Kramer DR, Winer JL, Pease BA, Amar AP, Mack WJ (2013) Cerebral vasospasm in traumatic brain injury. Neurol Res Int 2013:415813.

Kuehn R, Simard PF, Driscoll I, Keledjian K, Ivanova S, Tosun C, Williams A, Bochicchio G, Gerzanich V, Simard JM (2011) Rodent model of direct cranial blast injury. J Neurotrauma 28:2155-2169.

Kumar A, Stoica BA, Sabirzhanov B, Burns MP, Faden AI, Loane DJ (2013) Traumatic brain injury in aged animals increases lesion size and chronically alters microglial/macrophage classical and alternative activation states. Neurobiol Aging 34:1397-1411. 
Kumar RG, Diamond ML, Boles JA, Berger RP, Tisherman S, Kochanek PM, Wagner AK (2014) Acute CSF Interleukin-6 Trajectories after TBI: Associations with Neuroinflammation, Polytrauma, and Outcome. Brain Behav Immun.

LaPlaca MC, Thibault LE (1998) Dynamic mechanical deformation of neurons triggers an acute calcium response and cell injury involving the $\mathrm{N}$-methyl-D-aspartate glutamate receptor. J Neurosci Res 52:220-229.

LaPlaca MC, Cullen DK, McLoughlin JJ, Cargill RS, 2nd (2005) High rate shear strain of threedimensional neural cell cultures: a new in vitro traumatic brain injury model. $\mathrm{J}$ Biomech 38:1093-1105.

Larner SF, Hayes RL, Wang KK (2006) Unfolded protein response after neurotrauma. J Neurotrauma 23:807-829.

Larner SF, Hayes RL, McKinsey DM, Pike BR, Wang KK (2004) Increased expression and processing of caspase-12 after traumatic brain injury in rats. J Neurochem 88:78-90.

Laskowitz DT, Dawson HN (2014) Genetic influences and neuropathological sequelae of repetitive brain injury. Ann Neurol 75:617-618.

Laurer HL, Bareyre FM, Lee VM, Trojanowski JQ, Longhi L, Hoover R, Saatman KE, Raghupathi R, Hoshino S, Grady MS, McIntosh TK (2001) Mild head injury increasing the brain's vulnerability to a second concussive impact. J Neurosurg 95:859-870.

Lazaridis C, Czosnyka M (2012) Cerebral blood flow, brain tissue oxygen, and metabolic effects of decompressive craniectomy. Neurocrit Care 16:478-484.

Le Heron CJ, Wright SL, Melzer TR, Myall DJ, MacAskill MR, Livingston L, Keenan RJ, Watts R, Dalrymple-Alford JC, Anderson TJ (2014) Comparing cerebral perfusion in Alzheimer's disease and Parkinson's disease dementia: an ASL-MRI study. J Cereb Blood Flow Metab 34:964-970.

Lee HC, Chuang HC, Cho DY, Cheng KF, Lin PH, Chen CC (2010) Applying cerebral hypothermia and brain oxygen monitoring in treating severe traumatic brain injury. World Neurosurg 74:654-660.

Levy AS, Orlando A, Salottolo K, Mains CW, Bar-Or D (2013) Outcomes of a Nontransfer Protocol for Mild Traumatic Brain Injury with Abnormal Head Computed Tomography in a Rural Hospital Setting. World Neurosurg.

Li J, Siegel M, Yuan M, Zeng Z, Finnucan L, Persky R, Hurn PD, McCullough LD (2011) Estrogen enhances neurogenesis and behavioral recovery after stroke. J Cereb Blood Flow Metab 31:413-425.

Li N, Yang Y, Glover DP, Zhang J, Saraswati M, Robertson C, Pelled G (2013a) Evidence for Impaired Plasticity after Traumatic Brain Injury in the Developing Brain. J Neurotrauma.

Li S, Yang L, Selzer ME, Hu Y (2013b) Neuronal endoplasmic reticulum stress in axon injury and neurodegeneration. Ann Neurol 74:768-777.

Li S, Sun Y, Shan D, Feng B, Xing J, Duan Y, Dai J, Lei H, Zhou Y (2013c) Temporal profiles of axonal injury following impact acceleration traumatic brain injury in rats--a comparative study with diffusion tensor imaging and morphological analysis. Int J Legal Med 127:159-167.

Liao GP, Olson SD, Kota DJ, Hetz RA, Smith P, Bedi S, Cox CS, Jr. (2014) Far-red tracer analysis of traumatic cerebrovascular permeability. J Surg Res 190:628-633.

Liao Y, Liu P, Guo F, Zhang ZY, Zhang Z (2013) Oxidative burst of circulating neutrophils following traumatic brain injury in human. PLoS One 8:e68963. 
Ling G, Bandak F, Armonda R, Grant G, Ecklund J (2009) Explosive blast neurotrauma. J Neurotrauma 26:815-825.

Lipsky RH, Lin M (2015) Genetic predictors of outcome following traumatic brain injury. Handb Clin Neurol 127:23-41.

Liu CL, Li X, Hu GL, Li RJ, He YY, Zhong W, Li S, He KL, Wang LL (2012) Salubrinal protects against tunicamycin and hypoxia induced cardiomyocyte apoptosis via the PERK-eIF2alpha signaling pathway. J Geriatr Cardiol 9:258-268.

Liu HD, Li W, Chen ZR, Zhou ML, Zhuang Z, Zhang DD, Zhu L, Hang CH (2013) Increased expression of ferritin in cerebral cortex after human traumatic brain injury. Neurol Sci 34:1173-1180.

Liu MC, Kobeissy F, Zheng W, Zhang Z, Hayes RL, Wang KK (2011) Dual vulnerability of tau to calpains and caspase- 3 proteolysis under neurotoxic and neurodegenerative conditions. ASN Neuro 3:e00051.

Liu S, Yin F, Zhang J, Qian Y (2014a) The role of calpains in traumatic brain injury. Brain Inj 28:133-137.

Liu Y, Wang J, Qi SY, Ru LS, Ding C, Wang HJ, Zhao JS, Li JJ, Li AY, Wang DM (2014b) Reduced endoplasmic reticulum stress might alter the course of heart failure via caspase12 and JNK pathways. Can J Cardiol 30:368-375.

Liu Y, Liu Z, Li X, Luo B, Xiong J, Gan W, Jiang M, Zhang Z, Schluesener HJ, Zhang Z (2014c) Accumulation of connective tissue growth factor+ cells during the early phase of rat traumatic brain injury. Diagn Pathol 9:141.

Loane DJ, Kumar A (2015) Microglia in the TBI brain: The good, the bad, and the dysregulated. Exp Neurol.

Loane DJ, Stoica BA, Byrnes KR, Jeong W, Faden AI (2013) Activation of mGluR5 and inhibition of NADPH oxidase improves functional recovery after traumatic brain injury. J Neurotrauma 30:403-412.

Logsdon AF, Turner RC, Lucke-Wold BP, Robson MJ, Naser ZJ, Smith KE, Matsumoto RR, Huber JD, Rosen CL (2014) Altering endoplasmic reticulum stress in a model of blastinduced traumatic brain injury controls cellular fate and ameliorates neuropsychiatric symptoms. Front Cell Neurosci 8:421.

Long JB, Bentley TL, Wessner KA, Cerone C, Sweeney S, Bauman RA (2009) Blast overpressure in rats: recreating a battlefield injury in the laboratory. J Neurotrauma 26:827-840.

Longhi L, Saatman KE, Fujimoto S, Raghupathi R, Meaney DF, Davis J, McMillan BSA, Conte V, Laurer HL, Stein S, Stocchetti N, McIntosh TK (2005) Temporal window of vulnerability to repetitive experimental concussive brain injury. Neurosurgery 56:364374; discussion 364-374.

Lu J, Moochhala S, Kaur C, Ling E (2000) Changes in apoptosis-related protein (p53, Bax, Bcl-2 and Fos) expression with DNA fragmentation in the central nervous system in rats after closed head injury. Neurosci Lett 290:89-92.

Lucke-Wold BP, Turner RC, Logsdon AF, Bailes JE, Huber JD, Rosen CL (2014a) Linking traumatic brain injury to chronic traumatic encephalopathy: identification of potential mechanisms leading to neurofibrillary tangle development. J Neurotrauma 31:1129-1138.

Lucke-Wold BP, Turner RC, Logsdon AF, Bailes JE, Huber JD, Rosen CL (2014b) Linking Traumatic Brain Injury to Chronic Traumatic Encephalopathy: Identification of Potential Mechanisms Leading to Neurofibrillary Tangle Development. J Neurotrauma. 
Lucke-Wold BP, Logsdon AF, Smith KE, Turner RC, Alkon DL, Tan Z, Naser ZJ, Knotts CM, Huber JD, Rosen CL (2014c) Bryostatin-1 Restores Blood Brain Barrier Integrity following Blast-Induced Traumatic Brain Injury. Mol Neurobiol.

Lucke-Wold BP, Naser ZJ, Logsdon AF, Turner RC, Smith KE, Robson MJ, Bailes JE, Lee J, Rosen CL, Huber JD (2015a) Amelioration of NADPH-Mediated Stress Reduces Cell Death following Blast-induced Traumatic Brain Injury. Translational Research

In Press.

Lucke-Wold BP, Turner RC, Logsdon AF, Nguyen L, Bailes JE, Lee JM, Robson MJ, Omalu BI, Huber JD, Rosen CL (2015b) Endoplasmic reticulum stress implicated in chronic traumatic encephalopathy. J Neurosurg:1-16.

Lucke-Wold BP, Turner RC, Logsdon AF, Nguyen L, Bailes JE, Lee JM, Robson MJ, Omalu BI, Huber JD, Rosen CL (2015c) Endoplasmic reticulum stress implicated in chronic traumatic encephalopathy. Journal of Neurosurgery In Press.

Luo J, Nguyen A, Villeda S, Zhang H, Ding Z, Lindsey D, Bieri G, Castellano JM, Beaupre GS, Wyss-Coray T (2014) Long-term cognitive impairments and pathological alterations in a mouse model of repetitive mild traumatic brain injury. Front Neurol 5:12.

Ma J, Choi BR, Chung C, Min SS, Jeon WK, Han JS (2014) Chronic brain inflammation causes a reduction in GluN2A and GluN2B subunits of NMDA receptors and an increase in the phosphorylation of mitogen-activated protein kinases in the hippocampus. Mol Brain $7: 33$.

Ma M (2013) Role of calpains in the injury-induced dysfunction and degeneration of the mammalian axon. Neurobiol Dis 60:61-79.

Maas AI (2001) Neuroprotective agents in traumatic brain injury. Expert Opin Investig Drugs 10:753-767.

Maccioni RB, Otth C, Concha, II, Munoz JP (2001) The protein kinase Cdk5. Structural aspects, roles in neurogenesis and involvement in Alzheimer's pathology. Eur J Biochem 268:1518-1527.

Magnoni S, Brody DL (2010) New perspectives on amyloid-beta dynamics after acute brain injury: moving between experimental approaches and studies in the human brain. Arch Neurol 67:1068-1073.

Magnuson J, Leonessa F, Ling GS (2012) Neuropathology of explosive blast traumatic brain injury. Curr Neurol Neurosci Rep 12:570-579.

Malhotra JD, Kaufman RJ (2007) Endoplasmic reticulum stress and oxidative stress: a vicious cycle or a double-edged sword? Antioxid Redox Signal 9:2277-2293.

Mangat HS (2012) Severe traumatic brain injury. Continuum (Minneap Minn) 18:532-546.

Manley GT, Rosenthal G, Lam M, Morabito D, Yan D, Derugin N, Bollen A, Knudson MM, Panter SS (2006) Controlled cortical impact in swine: pathophysiology and biomechanics. J Neurotrauma 23:128-139.

Mannix R, Berglass J, Berkner J, Moleus P, Qiu J, Andrews N, Gunner G, Berglass L, Jantzie LL, Robinson S, Meehan WP, 3rd (2014) Chronic gliosis and behavioral deficits in mice following repetitive mild traumatic brain injury. J Neurosurg:1-9.

Mannix R, Meehan WP, Mandeville J, Grant PE, Gray T, Berglass J, Zhang J, Bryant J, Rezaie S, Chung JY, Peters NV, Lee C, Tien LW, Kaplan DL, Feany M, Whalen M (2013) Clinical correlates in an experimental model of repetitive mild brain injury. Ann Neurol 74:65-75. 
Marchesi VT (2014) Alzheimer's disease and CADASIL are heritable, adult-onset dementias that both involve damaged small blood vessels. Cell Mol Life Sci 71:949-955.

Maren S (2001) Neurobiology of Pavlovian fear conditioning. Annu Rev Neurosci 24:897-931. Marmarou CR, Liang X, Abidi NH, Parveen S, Taya K, Henderson SC, Young HF, Filippidis AS, Baumgarten CM (2014) Selective vasopressin-1a receptor antagonist prevents brain edema, reduces astrocytic cell swelling and GFAP, V1aR and AQP4 expression after focal traumatic brain injury. Brain Res.

Maroon JC, Winkelman R, Bost J, Amos A, Mathyssek C, Miele V (2015) Chronic traumatic encephalopathy in contact sports: a systematic review of all reported pathological cases. PLoS One 10:e0117338.

Mata-Mbemba D, Mugikura S, Nakagawa A, Murata T, Ishii K, Li L, Takase K, Kushimoto S, Takahashi S (2014a) Early CT findings to predict early death in patients with traumatic brain injury: Marshall and Rotterdam CT scoring systems compared in the major academic tertiary care hospital in northeastern Japan. Acad Radiol 21:605-611.

Mata-Mbemba D, Mugikura S, Nakagawa A, Murata T, Kato Y, Tatewaki Y, Li L, Takase K, Ishii K, Kushimoto S, Tominaga T, Takahashi S (2014b) Intraventricular Hemorrhage on Initial Computed Tomography as Marker of Diffuse Axonal Injury after Traumatic Brain Injury. J Neurotrauma.

Maughan PH, Scholten KJ, Schmidt RH (2000) Recovery of water maze performance in aged versus young rats after brain injury with the impact acceleration model. J Neurotrauma 17:1141-1153.

McCauley SR, Boake C, Levin HS, Contant CF, Song JX (2001) Postconcussional disorder following mild to moderate traumatic brain injury: anxiety, depression, and social support as risk factors and comorbidities. J Clin Exp Neuropsychol 23:792-808.

McCullough KD, Martindale JL, Klotz LO, Aw TY, Holbrook NJ (2001) Gadd153 sensitizes cells to endoplasmic reticulum stress by down-regulating Bcl 2 and perturbing the cellular redox state. Mol Cell Biol 21:1249-1259.

McKee AC, Daneshvar DH, Alvarez VE, Stein TD (2014) The neuropathology of sport. Acta Neuropathol 127:29-51.

McKee AC, Cantu RC, Nowinski CJ, Hedley-Whyte ET, Gavett BE, Budson AE, Santini VE, Lee HS, Kubilus CA, Stern RA (2009) Chronic traumatic encephalopathy in athletes: progressive tauopathy after repetitive head injury. J Neuropathol Exp Neurol 68:709-735.

McKee AC, Gavett BE, Stern RA, Nowinski CJ, Cantu RC, Kowall NW, Perl DP, HedleyWhyte ET, Price B, Sullivan C, Morin P, Lee HS, Kubilus CA, Daneshvar DH, Wulff M, Budson AE (2010) TDP-43 proteinopathy and motor neuron disease in chronic traumatic encephalopathy. J Neuropathol Exp Neurol 69:918-929.

McKee AC, Stern RA, Nowinski CJ, Stein TD, Alvarez VE, Daneshvar DH, Lee HS, Wojtowicz SM, Hall G, Baugh CM, Riley DO, Kubilus CA, Cormier KA, Jacobs MA, Martin BR, Abraham CR, Ikezu T, Reichard RR, Wolozin BL, Budson AE, Goldstein LE, Kowall NW, Cantu RC (2013) The spectrum of disease in chronic traumatic encephalopathy. Brain 136:43-64.

Medeiros R, Kitazawa M, Chabrier MA, Cheng D, Baglietto-Vargas D, Kling A, Moeller A, Green KN, LaFerla FM (2012) Calpain inhibitor A-705253 mitigates Alzheimer's disease-like pathology and cognitive decline in aged 3xTgAD mice. Am J Pathol 181:616-625. 
Meehan WP, 3rd, Zhang J, Mannix R, Whalen MJ (2012) Increasing recovery time between injuries improves cognitive outcome after repetitive mild concussive brain injuries in mice. Neurosurgery 71:885-891.

Mehan ND, Strauss KI (2012) Combined age- and trauma-related proteomic changes in rat neocortex: a basis for brain vulnerability. Neurobiol Aging 33:1857-1873.

Menon DK, Schwab K, Wright DW, Maas AI, Demographics, Clinical Assessment Working Group of the I, Interagency Initiative toward Common Data Elements for Research on Traumatic Brain I, Psychological H (2010) Position statement: definition of traumatic brain injury. Arch Phys Med Rehabil 91:1637-1640.

Methippara M, Mitrani B, Schrader FX, Szymusiak R, McGinty D (2012) Salubrinal, an endoplasmic reticulum stress blocker, modulates sleep homeostasis and activation of sleep- and wake-regulatory neurons. Neuroscience 209:108-118.

Mez J, Stern RA, McKee AC (2013) Chronic traumatic encephalopathy: where are we and where are we going? Curr Neurol Neurosci Rep 13:407.

Michael AP, Stout J, Roskos PT, Bolzenius J, Mogul D, Gfeller J, Bucholz R (2015) Evaluation of Cortical Thickness Following Traumatic Brain Injury in Military Veterans. J Neurotrauma.

Miller G (2012) Neuropathology. Blast injuries linked to neurodegeneration in veterans. Science 336:790-791.

Mills JD, Hadley K, Bailes JE (2011a) Dietary supplementation with the omega-3 fatty acid docosahexaenoic acid in traumatic brain injury. Neurosurgery 68:474-481; discussion 481.

Mills JD, Bailes JE, Sedney CL, Hutchins H, Sears B (2011b) Omega-3 fatty acid supplementation and reduction of traumatic axonal injury in a rodent head injury model. $\mathrm{J}$ Neurosurg 114:77-84.

Mills JD, Bailes JE, Turner RC, Dodson SC, Sakai J, Maroon JC (2012) Anabolic steroids and head injury. Neurosurgery 70:205-209; discussion 209-210.

Milman A, Rosenberg A, Weizman R, Pick CG (2005) Mild traumatic brain injury induces persistent cognitive deficits and behavioral disturbances in mice. J Neurotrauma 22:10031010.

Mitsis EM, Riggio S, Kostakoglu L, Dickstein DL, Machac J, Delman B, Goldstein M, Jennings D, D'Antonio E, Martin J, Naidich TP, Aloysi A, Fernandez C, Seibyl J, DeKosky ST, Elder GA, Marek K, Gordon W, Hof PR, Sano M, Gandy S (2014) Tauopathy PET and amyloid PET in the diagnosis of chronic traumatic encephalopathies: studies of a retired NFL player and of a man with FTD and a severe head injury. Transl Psychiatry 4:e441.

Miyauchi T, Wei EP, Povlishock JT (2013) Therapeutic targeting of the axonal and microvascular change associated with repetitive mild traumatic brain injury. $\mathrm{J}$ Neurotrauma 30:1664-1671.

Miyauchi T, Wei EP, Povlishock JT (2014) Evidence for the therapeutic efficacy of either mild hypothermia or oxygen radical scavengers after repetitive mild traumatic brain injury. $\mathbf{J}$ Neurotrauma 31:773-781.

Miyazaki S, Katayama Y, Lyeth BG, Jenkins LW, DeWitt DS, Goldberg SJ, Newlon PG, Hayes RL (1992) Enduring suppression of hippocampal long-term potentiation following traumatic brain injury in rat. Brain Res 585:335-339. 
Molino Y, Jabes F, Lacassagne E, Gaudin N, Khrestchatisky M (2014) Setting-up an in vitro model of rat blood-brain barrier (BBB): a focus on BBB impermeability and receptormediated transport. J Vis Exp:e51278.

Montenigro PH, Baugh CM, Daneshvar DH, Mez J, Budson AE, Au R, Katz DI, Cantu RC, Stern RA (2014) Clinical subtypes of chronic traumatic encephalopathy: literature review and proposed research diagnostic criteria for traumatic encephalopathy syndrome. Alzheimers Res Ther 6:68.

Moreno JA, Halliday M, Molloy C, Radford H, Verity N, Axten JM, Ortori CA, Willis AE, Fischer PM, Barrett DA, Mallucci GR (2013) Oral treatment targeting the unfolded protein response prevents neurodegeneration and clinical disease in prion-infected mice. Sci Transl Med 5:206ra138.

Moreno JA, Radford H, Peretti D, Steinert JR, Verity N, Martin MG, Halliday M, Morgan J, Dinsdale D, Ortori CA, Barrett DA, Tsaytler P, Bertolotti A, Willis AE, Bushell M, Mallucci GR (2012) Sustained translational repression by eIF2alpha-P mediates prion neurodegeneration. Nature 485:507-511.

Mori K (2000) Tripartite management of unfolded proteins in the endoplasmic reticulum. Cell 101:451-454.

Morrison B, 3rd, Cater HL, Benham CD, Sundstrom LE (2006) An in vitro model of traumatic brain injury utilising two-dimensional stretch of organotypic hippocampal slice cultures. J Neurosci Methods 150:192-201.

Mosienko V, Bert B, Beis D, Matthes S, Fink H, Bader M, Alenina N (2012) Exaggerated aggression and decreased anxiety in mice deficient in brain serotonin. Transl Psychiatry 2:e122.

Mouzon B, Chaytow H, Crynen G, Bachmeier C, Stewart J, Mullan M, Stewart W, Crawford F (2012) Repetitive mild traumatic brain injury in a mouse model produces learning and memory deficits accompanied by histological changes. J Neurotrauma 29:2761-2773.

Mouzon BC, Bachmeier C, Ferro A, Ojo JO, Crynen G, Acker CM, Davies P, Mullan M, Stewart W, Crawford F (2014) Chronic neuropathological and neurobehavioral changes in a repetitive mild traumatic brain injury model. Ann Neurol 75:241-254.

Mukhin AG, Ivanova SA, Allen JW, Faden AI (1998) Mechanical injury to neuronal/glial cultures in microplates: role of NMDA receptors and $\mathrm{pH}$ in secondary neuronal cell death. J Neurosci Res 51:748-758.

Munoz P, Zavala G, Castillo K, Aguirre P, Hidalgo C, Nunez MT (2006) Effect of iron on the activation of the MAPK/ERK pathway in PC12 neuroblastoma cells. Biol Res 39:189190.

Munoz P, Humeres A, Elgueta C, Kirkwood A, Hidalgo C, Nunez MT (2011) Iron mediates Nmethyl-D-aspartate receptor-dependent stimulation of calcium-induced pathways and hippocampal synaptic plasticity. J Biol Chem 286:13382-13392.

Mychasiuk R, Hehar H, van Waes L, Esser MJ (2014) Diet, age, and prior injury status differentially alter behavioral outcomes following concussion in rats. Neurobiol Dis.

Mychasiuk R, Hehar H, van Waes L, Esser MJ (2015) Diet, age, and prior injury status differentially alter behavioral outcomes following concussion in rats. Neurobiol Dis 73:111.

Nagy Z, Esiri MM, Smith AD (1997) Expression of cell division markers in the hippocampus in Alzheimer's disease and other neurodegenerative conditions. Acta Neuropathol 93:294300 . 
Nakagawa A, Manley GT, Gean AD, Ohtani K, Armonda R, Tsukamoto A, Yamamoto H, Takayama K, Tominaga T (2011) Mechanisms of primary blast-induced traumatic brain injury: insights from shock-wave research. J Neurotrauma 28:1101-1119.

Nakagawa T, Yuan J (2000) Cross-talk between two cysteine protease families. Activation of caspase-12 by calpain in apoptosis. J Cell Biol 150:887-894.

Nakagawa T, Zhu H, Morishima N, Li E, Xu J, Yankner BA, Yuan J (2000) Caspase-12 mediates endoplasmic-reticulum-specific apoptosis and cytotoxicity by amyloid-beta. Nature 403:98-103.

Nakka VP, Gusain A, Raghubir R (2010) Endoplasmic reticulum stress plays critical role in brain damage after cerebral ischemia/reperfusion in rats. Neurotox Res 17:189-202.

Needham CE, Ritzel D, Rule GT, Wiri S, Young L (2015) Blast Testing Issues and TBI: Experimental Models That Lead to Wrong Conclusions. Front Neurol 6:72.

Nemetz PN, Leibson C, Naessens JM, Beard M, Kokmen E, Annegers JF, Kurland LT (1999) Traumatic brain injury and time to onset of Alzheimer's disease: a population-based study. Am J Epidemiol 149:32-40.

Neuhof C, Neuhof H (2014) Calpain system and its involvement in myocardial ischemia and reperfusion injury. World J Cardiol 6:638-652.

Newcomb JK, Pike BR, Zhao X, Banik NL, Hayes RL (1999) Altered calpastatin protein levels following traumatic brain injury in rat. J Neurotrauma 16:1-11.

Niesman IR, Schilling JM, Shapiro LA, Kellerhals SE, Bonds JA, Kleschevnikov AM, Cui W, Voong A, Krajewski S, Ali SS, Roth DM, Patel HH, Patel PM, Head BP (2014) Traumatic brain injury enhances neuroinflammation and lesion volume in caveolin deficient mice. J Neuroinflammation 11:39.

Nijholt DA, van Haastert ES, Rozemuller AJ, Scheper W, Hoozemans JJ (2012) The unfolded protein response is associated with early tau pathology in the hippocampus of tauopathies. J Pathol 226:693-702.

Nirula R, Millar D, Greene T, McFadden M, Shah L, Scalea TM, Stein DM, Magnotti LJ, Jurkovich GJ, Vercruysse G, Demetriades D, Scherer LA, Peitzman A, Sperry J, Beauchamp K, Bell S, Feiz-Erfan I, O'Neill P, Coimbra R (2014) Decompressive craniectomy or medical management for refractory intracranial hypertension: an AASTMIT propensity score analysis. J Trauma Acute Care Surg 76:944-952; discussion 952945.

Nisenbaum EJ, Novikov DS, Lui YW (2014) The presence and role of iron in mild traumatic brain injury: an imaging perspective. J Neurotrauma 31:301-307.

O'Callaghan JP, Sriram K (2004) Focused microwave irradiation of the brain preserves in vivo protein phosphorylation: comparison with other methods of sacrifice and analysis of multiple phosphoproteins. J Neurosci Methods 135:159-168.

Ohri SS, Hetman M, Whittemore SR (2013) Restoring endoplasmic reticulum homeostasis improves functional recovery after spinal cord injury. Neurobiol Dis 58:29-37.

Ojo JO, Mouzon BC, Crawford F (2015) Repetitive head trauma, chronic traumatic encephalopathy and tau: Challenges in translating from mice to men. Exp Neurol.

Olsson Y, Rinder L, Lindgren S, Stalhammar D (1971) Studies on vascular permeability changes in experimental brain concussion. 3. A comparison between the effects of single and repeated sudden mechanical loading of the brain. Acta Neuropathol 19:225-233.

Omalu B (2014) Chronic traumatic encephalopathy. Prog Neurol Surg 28:38-49. 
Omalu B, Bailes J, Hamilton RL, Kamboh MI, Hammers J, Case M, Fitzsimmons R (2011a) Emerging histomorphologic phenotypes of chronic traumatic encephalopathy in American athletes. Neurosurgery 69:173-183; discussion 183.

Omalu B, Hammers JL, Bailes J, Hamilton RL, Kamboh MI, Webster G, Fitzsimmons RP (2011b) Chronic traumatic encephalopathy in an Iraqi war veteran with posttraumatic stress disorder who committed suicide. Neurosurg Focus 31:E3.

Omalu BI, Fitzsimmons RP, Hammers J, Bailes J (2010a) Chronic traumatic encephalopathy in a professional American wrestler. J Forensic Nurs 6:130-136.

Omalu BI, Bailes J, Hammers JL, Fitzsimmons RP (2010b) Chronic traumatic encephalopathy, suicides and parasuicides in professional American athletes: the role of the forensic pathologist. Am J Forensic Med Pathol 31:130-132.

Omalu BI, Hamilton RL, Kamboh MI, DeKosky ST, Bailes J (2010c) Chronic traumatic encephalopathy (CTE) in a National Football League Player: Case report and emerging medicolegal practice questions. J Forensic Nurs 6:40-46.

Omalu BI, DeKosky ST, Minster RL, Kamboh MI, Hamilton RL, Wecht CH (2005) Chronic traumatic encephalopathy in a National Football League player. Neurosurgery 57:128134; discussion 128-134.

Omalu BI, DeKosky ST, Hamilton RL, Minster RL, Kamboh MI, Shakir AM, Wecht CH (2006) Chronic traumatic encephalopathy in a national football league player: part II. Neurosurgery 59:1086-1092; discussion 1092-1083.

Osada N, Kosuge Y, Kihara T, Ishige K, Ito Y (2009) Apolipoprotein E-deficient mice are more vulnerable to ER stress after transient forebrain ischemia. Neurochem Int 54:403-409.

Otani N, Nawashiro H, Fukui S, Nomura N, Yano A, Miyazawa T, Shima K (2002) Differential activation of mitogen-activated protein kinase pathways after traumatic brain injury in the rat hippocampus. J Cereb Blood Flow Metab 22:327-334.

Padayachy LC, Rohlwink U, Zwane E, Fieggen G, Peter JC, Figaji AA (2012) The frequency of cerebral ischemia/hypoxia in pediatric severe traumatic brain injury. Childs Nerv Syst 28:1911-1918.

Page SJ, Yablon S (2003) Plasticity in TBI: concepts and applications. Foreword. Brain Inj 17:639-641.

Pandya JD, Pauly JR, Sullivan PG (2009) The optimal dosage and window of opportunity to maintain mitochondrial homeostasis following traumatic brain injury using the uncoupler FCCP. Exp Neurol 218:381-389.

Pandya JD, Pauly JR, Nukala VN, Sebastian AH, Day KM, Korde AS, Maragos WF, Hall ED, Sullivan PG (2007) Post-Injury Administration of Mitochondrial Uncouplers Increases Tissue Sparing and Improves Behavioral Outcome following Traumatic Brain Injury in Rodents. J Neurotrauma 24:798-811.

Panzer MB, Wood GW, Bass CR (2014) Scaling in neurotrauma: how do we apply animal experiments to people? Exp Neurol 261:120-126.

Panzer MB, Matthews KA, Yu AW, Morrison B, 3rd, Meaney DF, Bass CR (2012) A Multiscale Approach to Blast Neurotrauma Modeling: Part I - Development of Novel Test Devices for in vivo and in vitro Blast Injury Models. Front Neurol 3:46.

Paradis A, Zhang L (2013) Role of endothelin in uteroplacental circulation and fetal vascular function. Curr Vasc Pharmacol 11:594-605. 
Park E, Eisen R, Kinio A, Baker AJ (2013) Electrophysiological white matter dysfunction and association with neurobehavioral deficits following low-level primary blast trauma. Neurobiol Dis 52:150-159.

Park E, Gottlieb JJ, Cheung B, Shek PN, Baker AJ (2011) A model of low-level primary blast brain trauma results in cytoskeletal proteolysis and chronic functional impairment in the absence of lung barotrauma. J Neurotrauma 28:343-357.

Pechmann A, Anastasopoulos C, Korinthenberg R, van Velthoven-Wurster V, Kirschner J (2014) Decompressive Craniectomy after Severe Traumatic Brain Injury in Children: Complications and Outcome. Neuropediatrics.

Peek-Asa C, McArthur D, Hovda D, Kraus J (2001) Early predictors of mortality in penetrating compared with closed brain injury. Brain Inj 15:801-810.

Peskind ER, Brody D, Cernak I, McKee A, Ruff RL (2013) Military- and sports-related mild traumatic brain injury: clinical presentation, management, and long-term consequences. $\mathbf{J}$ Clin Psychiatry 74:180-188; quiz 188.

Petraglia AL, Plog BA, Dayawansa S, Dashnaw ML, Czerniecka K, Walker CT, Chen M, Hyrien O, Iliff JJ, Deane R, Huang JH, Nedergaard M (2014a) The pathophysiology underlying repetitive mild traumatic brain injury in a novel mouse model of chronic traumatic encephalopathy. Surg Neurol Int 5:184.

Petraglia AL, Plog BA, Dayawansa S, Chen M, Dashnaw ML, Czerniecka K, Walker CT, Viterise T, Hyrien O, Iliff JJ, Deane R, Nedergaard M, Huang JH (2014b) The spectrum of neurobehavioral sequelae after repetitive mild traumatic brain injury: a novel mouse model of chronic traumatic encephalopathy. J Neurotrauma 31:1211-1224.

Petraglia AL, Plog BA, Dayawansa S, Chen M, Dashnaw ML, Czerniecka K, Walker CT, Viterise T, Hyrien O, Iliff JJ, Deane R, Nedergaard M, Huang JH (2014c) The Spectrum of Neuro-behavioral Sequelae Following Repetitive Mild Traumatic Brain Injury: A Novel Mouse Model of Chronic Traumatic Encephalopathy (CTE). J Neurotrauma.

Plassman BL, Havlik RJ, Steffens DC, Helms MJ, Newman TN, Drosdick D, Phillips C, Gau BA, Welsh-Bohmer KA, Burke JR, Guralnik JM, Breitner JC (2000) Documented head injury in early adulthood and risk of Alzheimer's disease and other dementias. Neurology 55:1158-1166.

Plattner F, Angelo M, Giese KP (2006) The roles of cyclin-dependent kinase 5 and glycogen synthase kinase 3 in tau hyperphosphorylation. J Biol Chem 281:25457-25465.

Plattner F, Hernandéz, A., Kistler, T. M., Pozo, K., Zhong, P., Yuen, E., Hawasli, A., Cooke, S., Tan, C., Guo, A., Weiderhold, T., Yan, Z., Bibb, J.A (2014) Memory enhancement by targeting Cdk5 regulation of NR2B. Neuron in press.

Ponsford J, McLaren A, Schonberger M, Burke R, Rudzki D, Olver J, Ponsford M (2011) The association between apolipoprotein $\mathrm{E}$ and traumatic brain injury severity and functional outcome in a rehabilitation sample. J Neurotrauma 28:1683-1692.

Pop V, Badaut J (2011) A neurovascular perspective for long-term changes after brain trauma. Transl Stroke Res 2:533-545.

Prell T, Lautenschlager J, Weidemann L, Ruhmer J, Witte OW, Grosskreutz J (2014)

Endoplasmic reticulum stress is accompanied by activation of NF-kappaB in amyotrophic lateral sclerosis. J Neuroimmunol 270:29-36.

Prins ML, Hales A, Reger M, Giza CC, Hovda DA (2010) Repeat traumatic brain injury in the juvenile rat is associated with increased axonal injury and cognitive impairments. Dev Neurosci 32:510-518. 
Pun PB, Lu J, Moochhala S (2009) Involvement of ROS in BBB dysfunction. Free Radic Res 43:348-364.

Quan X, Wang J, Liang C, Zheng H, Zhang L (2015) Melatonin inhibits tunicamycin-induced endoplasmic reticulum stress and insulin resistance in skeletal muscle cells. Biochem Biophys Res Commun.

Qutub AA, Popel AS (2008) Reactive oxygen species regulate hypoxia-inducible factor 1alpha differentially in cancer and ischemia. Mol Cell Biol 28:5106-5119.

Rafaels KA, dale' Bass CR, Panzer MB, Salzar RS, Woods WA, Feldman SH, Walilko T, Kent RW, Capehart BP, Foster JB, Derkunt B, Toman A (2012) Brain injury risk from primary blast. J Trauma Acute Care Surg 73:895-901.

Raghupathi R, Margulies SS (2002) Traumatic axonal injury after closed head injury in the neonatal pig. J Neurotrauma 19:843-853.

Raghupathi R, Mehr MF, Helfaer MA, Margulies SS (2004) Traumatic axonal injury is exacerbated following repetitive closed head injury in the neonatal pig. J Neurotrauma 21:307-316.

Ramlackhansingh AF, Brooks DJ, Greenwood RJ, Bose SK, Turkheimer FE, Kinnunen KM, Gentleman S, Heckemann RA, Gunanayagam K, Gelosa G, Sharp DJ (2011) Inflammation after trauma: microglial activation and traumatic brain injury. Ann Neurol 70:374-383.

Razumovsky A, Tigno T, Hochheimer SM, Stephens FL, Bell R, Vo AH, Severson MA, Marshall SA, Oppenheimer SM, Ecker R, Armonda RA (2013) Cerebral hemodynamic changes after wartime traumatic brain injury. Acta Neurochir Suppl 115:87-90.

Readnower RD, Chavko M, Adeeb S, Conroy MD, Pauly JR, McCarron RM, Sullivan PG (2010) Increase in blood-brain barrier permeability, oxidative stress, and activated microglia in a rat model of blast-induced traumatic brain injury. J Neurosci Res 88:3530-3539.

Rebetez MM, Rochat L, Ghisletta P, Walder B, Van der Linden M (2015) Association between impulsivity, emotional/behavioural hyperactivation and functional outcome one year after severe traumatic brain injury. Brain Inj:1-7.

Reeves TM, Phillips LL, Povlishock JT (2005) Myelinated and unmyelinated axons of the corpus callosum differ in vulnerability and functional recovery following traumatic brain injury. Exp Neurol 196:126-137.

Reneer DV, Hisel RD, Hoffman JM, Kryscio RJ, Lusk BT, Geddes JW (2011) A multi-mode shock tube for investigation of blast-induced traumatic brain injury. J Neurotrauma 28:95-104.

Richmond DR, Damon EG, Fletcher ER, Bowen IG, White CS (1968) The relationship between selected blast-wave parameters and the response of mammals exposed to air blast. Ann $\mathrm{N}$ Y Acad Sci 152:103-121.

Rigg JL, Mooney SR (2011) Concussions and the military: issues specific to service members. PM R 3:S380-386.

Ringger NC, Tolentino PJ, McKinsey DM, Pike BR, Wang KK, Hayes RL (2004) Effects of injury severity on regional and temporal mRNA expression levels of calpains and caspases after traumatic brain injury in rats. J Neurotrauma 21:829-841.

Rink A, Fung KM, Trojanowski JQ, Lee VM, Neugebauer E, McIntosh TK (1995) Evidence of apoptotic cell death after experimental traumatic brain injury in the rat. Am J Pathol $147: 1575-1583$. 
Risling M, Davidsson J (2012) Experimental animal models for studies on the mechanisms of blast-induced neurotrauma. Front Neurol 3:30.

Ron D, Walter P (2007) Signal integration in the endoplasmic reticulum unfolded protein response. Nat Rev Mol Cell Biol 8:519-529.

Rosenbaum BP, Kelly ML, Kshettry VR, Weil RJ (2014) Neurologic disorders, in-hospital deaths, and years of potential life lost in the USA, 1988-2011. J Clin Neurosci.

Rosenfeld JV, Ford NL (2010) Bomb blast, mild traumatic brain injury and psychiatric morbidity: a review. Injury 41:437-443.

Rostami E, Engquist H, Enblad P (2014) Imaging of cerebral blood flow in patients with severe traumatic brain injury in the neurointensive care. Front Neurol 5:114.

Roth TL, Nayak D, Atanasijevic T, Koretsky AP, Latour LL, McGavern DB (2014) Transcranial amelioration of inflammation and cell death after brain injury. Nature 505:223-228.

Ruan J, Qi Z, Shen L, Jiang Y, Xu Y, Lan L, Luo L, Yin Z (2015) Crosstalk between JNK and NF-kappaB signaling pathways via HSP27 phosphorylation in HepG2 cells. Biochem Biophys Res Commun 456:122-128.

Rubenstein R, Chang B, Davies P, Wagner AMD, Robertson CS, Wang KK (2014) A Novel, Ultrasensitive Assay for Tau: Potential for Assessing Traumatic Brain Injury in Tissues and Biofluids. J Neurotrauma.

Rubino S, Zaman RA, Sturge CR, Fried JG, Desai A, Simmons NE, Lollis SS (2014) Outpatient follow-up of nonoperative cerebral contusion and traumatic subarachnoid hemorrhage: does repeat head CT alter clinical decision-making? J Neurosurg:1-6.

Rubovitch V, Shachar A, Werner H, Pick CG (2011a) Does IGF-1 administration after a mild traumatic brain injury in mice activate the adaptive arm of ER stress? Neurochem Int 58:443-446.

Rubovitch V, Barak S, Rachmany L, Goldstein RB, Zilberstein Y, Pick CG (2015) The neuroprotective effect of salubrinal in a mouse model of traumatic brain injury. Neuromolecular Med 17:58-70.

Rubovitch V, Ten-Bosch M, Zohar O, Harrison CR, Tempel-Brami C, Stein E, Hoffer BJ, Balaban CD, Schreiber S, Chiu WT, Pick CG (2011b) A mouse model of blast-induced mild traumatic brain injury. Exp Neurol 232:280-289.

Ruhe A, Fejer R, Gansslen A, Klein W (2014) Assessing postural stability in the concussed athlete: what to do, what to expect, and when. Sports Health 6:427-433.

Russell KL, Berman NE, Gregg PR, Levant B (2014) Fish oil improves motor function, limits blood-brain barrier disruption, and reduces Mmp9 gene expression in a rat model of juvenile traumatic brain injury. Prostaglandins Leukot Essent Fatty Acids 90:5-11.

Ryan NP, Catroppa C, Cooper JM, Beare R, Ditchfield M, Coleman L, Silk T, Crossley L, Rogers K, Beauchamp MH, Yeates KO, Anderson VA (2014) Relationships between acute imaging biomarkers and theory of mind impairment in post-acute pediatric traumatic brain injury: A prospective analysis using susceptibility weighted imaging (SWI). Neuropsychologia 66C:32-38.

Sabirzhanov B, Zhao Z, Stoica BA, Loane DJ, Wu J, Borroto C, Dorsey SG, Faden AI (2014) Downregulation of miR-23a and miR-27a following experimental traumatic brain injury induces neuronal cell death through activation of proapoptotic Bcl-2 proteins. J Neurosci 34:10055-10071. 
Sahin B, Kansy JW, Nairn AC, Spychala J, Ealick SE, Fienberg AA, Greene RW, Bibb JA (2004) Molecular characterization of recombinant mouse adenosine kinase and evaluation as a target for protein phosphorylation. Eur J Biochem 271:3547-3555.

Saito T, Shibasaki K, Kurachi M, Puentes S, Mikuni M, Ishizaki Y (2011) Cerebral capillary endothelial cells are covered by the VEGF-expressing foot processes of astrocytes. Neurosci Lett 497:116-121.

Sajja VS, Galloway M, Ghoddoussi F, Kepsel A, VandeVord P (2013) Effects of blast-induced neurotrauma on the nucleus accumbens. J Neurosci Res 91:593-601.

Salazar AM, Grafman J (2015) Post-traumatic epilepsy: clinical clues to pathogenesis and paths to prevention. Handb Clin Neurol 128:525-538.

Saljo A, Mayorga M, Bolouri H, Svensson B, Hamberger A (2011) Mechanisms and pathophysiology of the low-level blast brain injury in animal models. Neuroimage 54 Suppl 1:S83-88.

Salminen A, Kaarniranta K (2010) ER stress and hormetic regulation of the aging process. Ageing Res Rev 9:211-217.

Salminen A, Kauppinen A, Suuronen T, Kaarniranta K, Ojala J (2009) ER stress in Alzheimer's disease: a novel neuronal trigger for inflammation and Alzheimer's pathology. $\mathbf{J}$ Neuroinflammation 6:41.

Sanelli PC, Pandya A, Segal AZ, Gupta A, Hurtado-Rua S, Ivanidze J, Kesavabhotla K, Mir D, Mushlin AI, Hunink MG (2014) Cost-Effectiveness of CT Angiography and Perfusion Imaging for Delayed Cerebral Ischemia and Vasospasm in Aneurysmal Subarachnoid Hemorrhage. AJNR Am J Neuroradiol.

Sano R, Reed JC (2013) ER stress-induced cell death mechanisms. Biochim Biophys Acta 1833:3460-3470.

Saulle M, Greenwald BD (2012) Chronic traumatic encephalopathy: a review. Rehabil Res Pract 2012:816069.

Schafer DP, Jha S, Liu F, Akella T, McCullough LD, Rasband MN (2009) Disruption of the axon initial segment cytoskeleton is a new mechanism for neuronal injury. J Neurosci 29:13242-13254.

Schaible EV, Windschugl J, Bobkiewicz W, Kaburov Y, Dangel L, Kramer T, Huang C, Sebastiani A, Luh C, Werner C, Engelhard K, Thal SC, Schafer MK (2014) 2Methoxyestradiol confers neuroprotection and inhibits a maladaptive HIF-1alpha response after traumatic brain injury in mice. J Neurochem 129:940-954.

Scheibel RS, Newsome MR, Troyanskaya M, Lin X, Steinberg JL, Radaideh M, Levin HS (2012) Altered brain activation in military personnel with one or more traumatic brain injuries following blast. J Int Neuropsychol Soc 18:89-100.

Scheper W, Hoozemans JJ (2013) A new PERKspective on neurodegeneration. Sci Transl Med 5:206fs 237.

Scheper W, Nijholt DA, Hoozemans JJ (2011) The unfolded protein response and proteostasis in Alzheimer disease: preferential activation of autophagy by endoplasmic reticulum stress. Autophagy 7:910-911.

Schiera G, Bono E, Raffa MP, Gallo A, Pitarresi GL, Di Liegro I, Savettieri G (2003) Synergistic effects of neurons and astrocytes on the differentiation of brain capillary endothelial cells in culture. J Cell Mol Med 7:165-170.

Schmitz C, Hof PR (2005) Design-based stereology in neuroscience. Neuroscience 130:813-831. 
Schmued LC, Hopkins KJ (2000) Fluoro-Jade B: a high affinity fluorescent marker for the localization of neuronal degeneration. Brain Res 874:123-130.

Schneiderman AI, Braver ER, Kang HK (2008) Understanding sequelae of injury mechanisms and mild traumatic brain injury incurred during the conflicts in Iraq and Afghanistan: persistent postconcussive symptoms and posttraumatic stress disorder. Am J Epidemiol 167:1446-1452.

Schroeter ML, Ettrich B, Schwier C, Scheid R, Guthke T, von Cramon DY (2007) Diffuse axonal injury due to traumatic brain injury alters inhibition of imitative response tendencies. Neuropsychologia 45:3149-3156.

Schwarzbold ML, Rial D, De Bem T, Machado DG, Cunha MP, dos Santos AA, dos Santos DB, Figueiredo CP, Farina M, Goldfeder EM, Rodrigues AL, Prediger RD, Walz R (2010) Effects of traumatic brain injury of different severities on emotional, cognitive, and oxidative stress-related parameters in mice. J Neurotrauma 27:1883-1893.

Shahlaie K, Keachie K, Hutchins IM, Rudisill N, Madden LK, Smith KA, Ko KA, Latchaw RE, Muizelaar JP (2011) Risk factors for posttraumatic vasospasm. J Neurosurg 115:602-611.

Sharma P, Su YA, Barry ES, Grunberg NE, Lei Z (2012) Mitochondrial targeted neuron focused genes in hippocampus of rats with traumatic brain injury. Int J Crit Illn Inj Sci 2:172-179.

Shin SS, Pathak S, Presson N, Bird W, Wagener L, Schneider W, Okonkwo DO, FernandezMiranda JC (2014) Detection of white matter injury in concussion using high-definition fiber tractography. Prog Neurol Surg 28:86-93.

Shitaka Y, Tran HT, Bennett RE, Sanchez L, Levy MA, Dikranian K, Brody DL (2011) Repetitive closed-skull traumatic brain injury in mice causes persistent multifocal axonal injury and microglial reactivity. J Neuropathol Exp Neurol 70:551-567.

Shohami E, Biegon A (2014) Novel Approach to the Role of NMDA Receptors in Traumatic Brain Injury. CNS Neurol Disord Drug Targets 13:567-573.

Shridharani JK, Wood GW, Panzer MB, Capehart BP, Nyein MK, Radovitzky RA, Bass CR (2012) Porcine head response to blast. Front Neurol 3:70.

Sieg F, Wahle P, Pape HC (1999) Cellular reactivity to mechanical axonal injury in an organotypic in vitro model of neurotrauma. J Neurotrauma 16:1197-1213.

Simpkins JW, Green PS, Gridley KE, Singh M, de Fiebre NC, Rajakumar G (1997) Role of estrogen replacement therapy in memory enhancement and the prevention of neuronal loss associated with Alzheimer's disease. Am J Med 103:19S-25S.

Sinclair RA, Burns J, Dunnill MS (1981) Immunoperoxidase staining of formalin-fixed, paraffinembedded, human renal biopsies with a comparison of the peroxidase-antiperoxidase (PAP) and indirect methods. J Clin Pathol 34:859-865.

Sivanandam TM, Thakur MK (2012) Traumatic brain injury: a risk factor for Alzheimer's disease. Neurosci Biobehav Rev 36:1376-1381.

Slemmer JE, Weber JT (2005) The extent of damage following repeated injury to cultured hippocampal cells is dependent on the severity of insult and inter-injury interval. Neurobiol Dis 18:421-431.

Slemmer JE, Weber JT, De Zeeuw CI (2004) Cell death, glial protein alterations and elevated S100 beta release in cerebellar cell cultures following mechanically induced trauma. Neurobiol Dis 15:563-572.

Small GW, Kepe V, Siddarth P, Ercoli LM, Merrill DA, Donoghue N, Bookheimer SY, Martinez J, Omalu B, Bailes J, Barrio JR (2013) PET scanning of brain tau in retired national football league players: preliminary findings. Am J Geriatr Psychiatry 21:138-144. 
Smith DH, Johnson VE, Stewart W (2013) Chronic neuropathologies of single and repetitive TBI: substrates of dementia? Nat Rev Neurol 9:211-221.

Smith DH, Chen XH, Iwata A, Graham DI (2003) Amyloid beta accumulation in axons after traumatic brain injury in humans. J Neurosurg 98:1072-1077.

Smith DH, Soares HD, Pierce JS, Perlman KG, Saatman KE, Meaney DF, Dixon CE, McIntosh TK (1995) A model of parasagittal controlled cortical impact in the mouse: cognitive and histopathologic effects. J Neurotrauma 12:169-178.

Smith DW, Bailes JE, Fisher JA, Robles J, Turner RC, Mills JD (2012) Internal jugular vein compression mitigates traumatic axonal injury in a rat model by reducing the intracranial slosh effect. Neurosurgery 70:740-746.

Sokka AL, Putkonen N, Mudo G, Pryazhnikov E, Reijonen S, Khiroug L, Belluardo N, Lindholm D, Korhonen L (2007) Endoplasmic reticulum stress inhibition protects against excitotoxic neuronal injury in the rat brain. J Neurosci 27:901-908.

Sominsky L, Walker AK, Hodgson DM (2015) Editorial: Neuroinflammation and behavior. Front Neurosci 9:201.

Sosa MA, De Gasperi R, Paulino AJ, Pricop PE, Shaughness MC, Maudlin-Jeronimo E, Hall AA, Janssen WG, Yuk FJ, Dorr NP, Dickstein DL, McCarron RM, Chavko M, Hof PR, Ahlers ST, Elder GA (2013) Blast overpressure induces shear-related injuries in the brain of rats exposed to a mild traumatic brain injury. Acta Neuropathol Commun 1:51.

Stein TD, Alvarez VE, McKee AC (2014) Chronic traumatic encephalopathy: a spectrum of neuropathological changes following repetitive brain trauma in athletes and military personnel. Alzheimers Res Ther 6:4.

Stern RA, Riley DO, Daneshvar DH, Nowinski CJ, Cantu RC, McKee AC (2011) Long-term consequences of repetitive brain trauma: chronic traumatic encephalopathy. PM R 3:S460-467.

Stern RA, Daneshvar DH, Baugh CM, Seichepine DR, Montenigro PH, Riley DO, Fritts NG, Stamm JM, Robbins CA, McHale L, Simkin I, Stein TD, Alvarez VE, Goldstein LE, Budson AE, Kowall NW, Nowinski CJ, Cantu RC, McKee AC (2013) Clinical presentation of chronic traumatic encephalopathy. Neurology 81:1122-1129.

Streit WJ, Mrak RE, Griffin WS (2004) Microglia and neuroinflammation: a pathological perspective. J Neuroinflammation 1:14.

Susman M, DiRusso SM, Sullivan T, Risucci D, Nealon P, Cuff S, Haider A, Benzil D (2002) Traumatic brain injury in the elderly: increased mortality and worse functional outcome at discharge despite lower injury severity. J Trauma 53:219-223; discussion 223-214.

Sutton RL, Lescaudron L, Stein DG (1993) Unilateral cortical contusion injury in the rat: vascular disruption and temporal development of cortical necrosis. J Neurotrauma 10:135-149.

Suzuki S, Gerhold LM, Bottner M, Rau SW, Dela Cruz C, Yang E, Zhu H, Yu J, Cashion AB, Kindy MS, Merchenthaler I, Gage FH, Wise PM (2007) Estradiol enhances neurogenesis following ischemic stroke through estrogen receptors alpha and beta. J Comp Neurol 500:1064-1075.

Svetlov SI, Prima V, Glushakova O, Svetlov A, Kirk DR, Gutierrez H, Serebruany VL, Curley KC, Wang KK, Hayes RL (2012) Neuro-glial and systemic mechanisms of pathological responses in rat models of primary blast overpressure compared to "composite" blast. Front Neurol 3:15. 
Szegezdi E, Logue SE, Gorman AM, Samali A (2006) Mediators of endoplasmic reticulum stress-induced apoptosis. EMBO Rep 7:880-885.

Szmydynger-Chodobska J, Fox LM, Lynch KM, Zink BJ, Chodobski A (2010) Vasopressin amplifies the production of proinflammatory mediators in traumatic brain injury. $\mathrm{J}$ Neurotrauma 27:1449-1461.

Szmydynger-Chodobska J, Chung I, Kozniewska E, Tran B, Harrington FJ, Duncan JA, Chodobski A (2004) Increased expression of vasopressin v1a receptors after traumatic brain injury. J Neurotrauma 21:1090-1102.

Tabas I, Ron D (2011) Integrating the mechanisms of apoptosis induced by endoplasmic reticulum stress. Nat Cell Biol 13:184-190.

Taber KH, Warden DL, Hurley RA (2006) Blast-related traumatic brain injury: what is known? J Neuropsychiatry Clin Neurosci 18:141-145.

Talavage TM, Nauman EA, Breedlove EL, Yoruk U, Dye AE, Morigaki KE, Feuer H, Leverenz LJ (2014) Functionally-detected cognitive impairment in high school football players without clinically-diagnosed concussion. J Neurotrauma 31:327-338.

Tartaglia MC, Hazrati LN, Davis KD, Green RE, Wennberg R, Mikulis D, Ezerins LJ, Keightley M, Tator C (2014) Chronic traumatic encephalopathy and other neurodegenerative proteinopathies. Front Hum Neurosci 8:30.

Tavazzi B, Signoretti S, Lazzarino G, Amorini AM, Delfini R, Cimatti M, Marmarou A, Vagnozzi R (2005) Cerebral oxidative stress and depression of energy metabolism correlate with severity of diffuse brain injury in rats. Neurosurgery 56:582-589; discussion 582-589.

Terrio H, Brenner LA, Ivins BJ, Cho JM, Helmick K, Schwab K, Scally K, Bretthauer R, Warden D (2009) Traumatic brain injury screening: preliminary findings in a US Army Brigade Combat Team. J Head Trauma Rehabil 24:14-23.

Theadom A, Parmar P, Jones K, Barker-Collo S, Starkey N, McPherson K, Ameratunga S, Feigin VL (2014) Frequency and impact of recurrent traumatic brain injury in a population-based sample. J Neurotrauma.

Timaru-Kast R, Luh C, Gotthardt P, Huang C, Schafer MK, Engelhard K, Thal SC (2012) Influence of age on brain edema formation, secondary brain damage and inflammatory response after brain trauma in mice. PLoS One 7:e43829.

Titus DJ, Furones C, Kang Y, Atkins CM (2013) Age-dependent alterations in cAMP signaling contribute to synaptic plasticity deficits following traumatic brain injury. Neuroscience 231:182-194.

Toklu HZ, Tumer N (2015) Oxidative Stress, Brain Edema, Blood-Brain Barrier Permeability, and Autonomic Dysfunction from Traumatic Brain Injury. In: Brain Neurotrauma: Molecular, Neuropsychological, and Rehabilitation Aspects (Kobeissy FHP, ed). Boca Raton (FL).

Tompkins P, Tesiram Y, Lerner M, Gonzalez LP, Lightfoot S, Rabb CH, Brackett DJ (2013) Brain injury: neuro-inflammation, cognitive deficit, and magnetic resonance imaging in a model of blast induced traumatic brain injury. J Neurotrauma 30:1888-1897.

Tran HT, Sanchez L, Brody DL (2012) Inhibition of JNK by a peptide inhibitor reduces traumatic brain injury-induced tauopathy in transgenic mice. J Neuropathol Exp Neurol 71:116-129.

Tran HT, LaFerla FM, Holtzman DM, Brody DL (2011) Controlled cortical impact traumatic brain injury in 3xTg-AD mice causes acute intra-axonal amyloid-beta accumulation and 
independently accelerates the development of tau abnormalities. J Neurosci 31:95139525.

Truettner JS, Hu B, Alonso OF, Bramlett HM, Kokame K, Dietrich WD (2007) Subcellular stress response after traumatic brain injury. J Neurotrauma 24:599-612.

Turner RC, Dodson SC, Rosen CL, Huber JD (2013a) The science of cerebral ischemia and the quest for neuroprotection: navigating past failure to future success. J Neurosurg 118:1072-1085.

Turner RC, Lucke-Wold BP, Robson MJ, Omalu BI, Petraglia AL, Bailes JE (2012a) Repetitive traumatic brain injury and development of chronic traumatic encephalopathy: a potential role for biomarkers in diagnosis, prognosis, and treatment? Front Neurol 3:186.

Turner RC, Naser ZJ, Bailes JE, Smith DW, Fisher JA, Rosen CL (2012b) Effect of slosh mitigation on histologic markers of traumatic brain injury: laboratory investigation. $\mathrm{J}$ Neurosurg 117:1110-1118.

Turner RC, Naser ZJ, Bailes JE, Smith DW, Fisher JA, Rosen CL (2012c) Effect of slosh mitigation on histologic markers of traumatic brain injury. J Neurosurg.

Turner RC, Seminerio MJ, Naser ZJ, Ford JN, Martin SJ, Matsumoto RR, Rosen CL, Huber JD (2012d) Effects of aging on behavioral assessment performance: implications for clinically relevant models of neurological disease. J Neurosurg 117:629-637.

Turner RC, VanGilder RL, Naser ZJ, Lucke-Wold BP, Bailes JE, Matsumoto RR, Huber JD, Rosen CL (2014) Elucidating the severity of preclinical traumatic brain injury models: a role for functional assessment? Neurosurgery 74:382-394; discussion 394.

Turner RC, Naser ZJ, Logsdon AF, DiPasquale KH, Jackson GJ, Robson MJ, Gettens RT, Matsumoto RR, Huber JD, Rosen CL (2013b) Modeling clinically relevant blast parameters based on scaling principles produces functional \& histological deficits in rats. Exp Neurol 248:520-529.

Tweedie D, Rachmany L, Rubovitch V, Zhang Y, Becker KG, Perez E, Hoffer BJ, Pick CG, Greig NH (2013) Changes in mouse cognition and hippocampal gene expression observed in a mild physical- and blast-traumatic brain injury. Neurobiol Dis 54:1-11.

Ueda Y, Walker SA, Povlishock JT (2006) Perivascular nerve damage in the cerebral circulation following traumatic brain injury. Acta Neuropathol 112:85-94.

Uranga RM, Giusto NM, Salvador GA (2009) Iron-induced oxidative injury differentially regulates PI3K/Akt/GSK3beta pathway in synaptic endings from adult and aged rats. Toxicol Sci 111:331-344.

Urano F, Wang X, Bertolotti A, Zhang Y, Chung P, Harding HP, Ron D (2000) Coupling of stress in the ER to activation of JNK protein kinases by transmembrane protein kinase IRE1. Science 287:664-666.

Urra H, Dufey E, Lisbona F, Rojas-Rivera D, Hetz C (2013) When ER stress reaches a dead end. Biochim Biophys Acta 1833:3507-3517.

Uryu K, Laurer H, McIntosh T, Pratico D, Martinez D, Leight S, Lee VM, Trojanowski JQ (2002) Repetitive mild brain trauma accelerates Abeta deposition, lipid peroxidation, and cognitive impairment in a transgenic mouse model of Alzheimer amyloidosis. J Neurosci 22:446-454.

Vaishnavi S, Rao V, Fann JR (2009) Neuropsychiatric problems after traumatic brain injury: unraveling the silent epidemic. Psychosomatics 50:198-205.

Van Den Heuvel C, Thornton E, Vink R (2007) Traumatic brain injury and Alzheimer's disease: a review. Prog Brain Res 161:303-316. 
van der Eerden AW, Khalilzadeh O, Perlbarg V, Dinkel J, Sanchez P, Vos PE, Luyt CE, Stevens RD, Menjot de Champfleur N, Delmaire C, Tollard E, Gupta R, Dormont D, Laureys S, Benali H, Vanhaudenhuyse A, Galanaud D, Puybasset L, Consortium N (2014) White matter changes in comatose survivors of anoxic ischemic encephalopathy and traumatic brain injury: comparative diffusion-tensor imaging study. Radiology 270:506-516.

Wagner AK, Bayir H, Ren D, Puccio A, Zafonte RD, Kochanek PM (2004a) Relationships between cerebrospinal fluid markers of excitotoxicity, ischemia, and oxidative damage after severe TBI: the impact of gender, age, and hypothermia. J Neurotrauma 21:125-136.

Wagner AK, Willard LA, Kline AE, Wenger MK, Bolinger BD, Ren D, Zafonte RD, Dixon CE (2004b) Evaluation of estrous cycle stage and gender on behavioral outcome after experimental traumatic brain injury. Brain Res 998:113-121.

Wali B, Sayeed I, Stein DG (2011) Improved behavioral outcomes after progesterone administration in aged male rats with traumatic brain injury. Restor Neurol Neurosci 29:61-71.

Walter P, Ron D (2011) The unfolded protein response: from stress pathway to homeostatic regulation. Science 334:1081-1086.

Wang G, Shi Y, Jiang X, Leak RK, Hu X, Wu Y, Pu H, Li WW, Tang B, Wang Y, Gao Y, Zheng P, Bennett MV, Chen J (2015) HDAC inhibition prevents white matter injury by modulating microglia/macrophage polarization through the GSK3beta/PTEN/Akt axis. Proc Natl Acad Sci U S A 112:2853-2858.

Wang KK, Larner SF, Robinson G, Hayes RL (2006) Neuroprotection targets after traumatic brain injury. Curr Opin Neurol 19:514-519.

Wang Y, Arun P, Wei Y, Oguntayo S, Gharavi R, Valiyaveettil M, Nambiar MP, Long JB (2014) Repeated blast exposures cause brain DNA fragmentation in mice. J Neurotrauma 31:498-504.

Weber JT (2007) Experimental models of repetitive brain injuries. Prog Brain Res 161:253-261.

Webster SJ, Van Eldik LJ, Watterson DM, Bachstetter AD (2015) Closed head injury in an agerelated Alzheimer mouse model leads to an altered neuroinflammatory response and persistent cognitive impairment. J Neurosci 35:6554-6569.

Weil ZM, Gaier KR, Karelina K (2014) Injury timing alters metabolic, inflammatory and functional outcomes following repeated mild traumatic brain injury. Neurobiol Dis 70:108-116.

Weiner MW (2013) Dementia in 2012: Further insights into Alzheimer disease pathogenesis. Nat Rev Neurol 9:65-66.

Werner C, Engelhard K (2007) Pathophysiology of traumatic brain injury. Br J Anaesth 99:4-9.

Wiescholleck V, Manahan-Vaughan D (2013a) Long-lasting changes in hippocampal synaptic plasticity and cognition in an animal model of NMDA receptor dysfunction in psychosis. Neuropharmacology 74:48-58.

Wiescholleck V, Manahan-Vaughan D (2013b) Persistent deficits in hippocampal synaptic plasticity accompany losses of hippocampus-dependent memory in a rodent model of psychosis. Front Integr Neurosci 7:12.

Wilberger JE, Jr., Harris M, Diamond DL (1991) Acute subdural hematoma: morbidity, mortality, and operative timing. J Neurosurg 74:212-218.

Wilson CD, Shankar JJ (2014) Diagnosing Vasospasm After Subarachnoid Hemorrhage: CTA and CTP. Can J Neurol Sci 41:314-319. 
Wu A, Ying Z, Gomez-Pinilla F (2004) Dietary omega-3 fatty acids normalize BDNF levels, reduce oxidative damage, and counteract learning disability after traumatic brain injury in rats. J Neurotrauma 21:1457-1467.

Wu J, Zhao Z, Sabirzhanov B, Stoica BA, Kumar A, Luo T, Skovira J, Faden AI (2014) Spinal cord injury causes brain inflammation associated with cognitive and affective changes: role of cell cycle pathways. J Neurosci 34:10989-11006.

Xiong Y, Mahmood A, Chopp M (2013) Animal models of traumatic brain injury. Nat Rev Neurosci 14:128-142.

Xu FF, Sun S, Ho AS, Lee D, Kiang KM, Zhang XQ, Wang AM, Wu EX, Lui WM, Liu BY, Leung GK (2014a) Effects of progesterone vs. dexamethasone on brain oedema and inflammatory responses following experimental brain resection. Brain Inj:1-8.

Xu ZS, Yao A, Chu SS, Paun MK, McClintic AM, Murphy SP, Mourad PD (2014b) Detection of mild traumatic brain injury in rodent models using shear wave elastography: preliminary studies. J Ultrasound Med 33:1763-1771.

Yang F, Zhang X, Sun Y, Wang B, Zhou C, Luo Y, Ge P (2013) Ischemic postconditioning decreases cerebral edema and brain blood barrier disruption caused by relief of carotid stenosis in a rat model of cerebral hypoperfusion. PLoS One 8:e57869.

Yeh DD, Schecter WP (2012) Primary blast injuries--an updated concise review. World J Surg 36:966-972.

Yen LF, Wei VC, Kuo EY, Lai TW (2013) Distinct patterns of cerebral extravasation by Evans blue and sodium fluorescein in rats. PLoS One 8:e68595.

Yeoh S, Bell ED, Monson KL (2013) Distribution of blood-brain barrier disruption in primary blast injury. Ann Biomed Eng 41:2206-2214.

Yi JH, Hazell AS (2006) Excitotoxic mechanisms and the role of astrocytic glutamate transporters in traumatic brain injury. Neurochem Int 48:394-403.

Yoshiyama Y, Uryu K, Higuchi M, Longhi L, Hoover R, Fujimoto S, McIntosh T, Lee VM, Trojanowski JQ (2005) Enhanced neurofibrillary tangle formation, cerebral atrophy, and cognitive deficits induced by repetitive mild brain injury in a transgenic tauopathy mouse model. J Neurotrauma 22:1134-1141.

Zaloshnja E, Miller T, Langlois JA, Selassie AW (2008) Prevalence of long-term disability from traumatic brain injury in the civilian population of the United States, 2005. J Head Trauma Rehabil 23:394-400.

Zander NE, Piehler T, Boggs ME, Banton R, Benjamin R (2015) In vitro studies of primary explosive blast loading on neurons. J Neurosci Res 93:1353-1363.

Zhang J, Teng Z, Song Y, Hu M, Chen C (2015) Inhibition of monoacylglycerol lipase prevents chronic traumatic encephalopathy-like neuropathology in a mouse model of repetitive mild closed head injury. J Cereb Blood Flow Metab 35:706.

Zhang K, Kaufman RJ (2008) From endoplasmic-reticulum stress to the inflammatory response. Nature 454:455-462.

Zhang QG, Laird MD, Han D, Nguyen K, Scott E, Dong Y, Dhandapani KM, Brann DW (2012) Critical role of NADPH oxidase in neuronal oxidative damage and microglia activation following traumatic brain injury. PLoS One 7:e34504.

Zhang Y, Liu W, Zhou Y, Ma C, Li S, Cong B (2014a) Endoplasmic reticulum stress is involved in restraint stress-induced hippocampal apoptosis and cognitive impairments in rats. Physiol Behav 131:41-48. 
Zhang YP, Cai J, Shields LB, Liu N, Xu XM, Shields CB (2014b) Traumatic brain injury using mouse models. Transl Stroke Res 5:454-471.

Zhong N, Ramaswamy G, Weisgraber KH (2009) Apolipoprotein E4 domain interaction induces endoplasmic reticulum stress and impairs astrocyte function. J Biol Chem 284:2727327280.

Zhu F, Skelton P, Chou CC, Mao H, Yang KH, King AI (2013) Biomechanical responses of a pig head under blast loading: a computational simulation. Int J Numer Method Biomed Eng 29:392-407.

Zhu F, Mao H, Dal Cengio Leonardi A, Wagner C, Chou C, Jin X, Bir C, Vandevord P, Yang KH, King AI (2010) Development of an FE model of the rat head subjected to air shock loading. Stapp Car Crash J 54:211-225. 


\section{ARIC FLINT LOGSDON}

logsdoa@gmail.com

(814) $720-3676$

EDUCATION

WeSt ViRgINIA UNIVERSITY, Morgantown, WV

$\mathrm{PhD}$ Candidate Pharmaceutical Sciences

GPA 3.5

Fall 2011-Spring 2016

"Elucidating mechanisms of neurobehavioral dysfunction in a rodent model of traumatic brain injury"

ALLEGHENY COLLEGE, Meadville, PA

Bachelor of Science Neuroscience

GPA 3.3

Fall 2006-Spring 2010

"Investigating the role of norepinephrine in a rodent model of Parkinson's disease"

\section{HONORS \& AWARDS}

PREDOCTORAL FELlOWSHIP in PHARMACEUTICAL SCIENCE AMERICAN FOUNDATION for PHARMACEUTICAL EDUCATION

Spring 2016

PRDOCTORAL FELlOWSHIP in PHARMACEUTICAL SCIENCE

AMERICAN FOUNDATION for PHARMACEUTICAL EDUCATION

Spring 2015

Second Place Poster PResentation

Fall 2015

APPALACHIAN REGIONAL CELl CONFERENCE | Regional Conference

PHI LAMBDA SIGMA LEADERSHIP SOCIETY

Fall 2015

WEST VIRGINIA UNIVERSITY | School of Pharmacy

RHO CHI HONOR SOCIETY

WeSt VIRGINIA UNIVERSITY | School of Pharmacy

Spring 2015

GRADUATE STUDENT RESEARCH AWARD

WEST VIRGINIA UNIVERSITY | School of Pharmacy

Spring 2015

First Place Poster PResentation

Fall 2014

AMERICAN ASSOCIATION of PHARMACEUTICAL SCIENTISTS | Regional Conference

Second Place Poster Presentation

VAN LIERE CONVOCATION | Local Conference

Spring 2014

\section{PROFESSIONAL SERVICE}

GRAduAte TEACHING Assistant | Neuropharmacology Journal Club | Jason D Huber PhD Fall 2015 WEST VIRGINIA UNIVERSITY | Department of Pharmaceutical Sciences

Graduate Teaching Assistant | Advanced Pharmacology 2 | Jason D Huber PhD S Spring 2015 WEST VIRGINIA UNIVERSITY | Department of Pharmaceutical Sciences

Undergraduate Teaching Assistant | Neurophysiology | Jeffrey Cross PhD $\quad$ Spring 2009-2010 ALLEGHENY COLLEGE | Department of Neuroscience 


\section{TECHNICAL SKILLS}

COMPUTER

C/C++, Java, MatLab, Python, SPSS, Image J, Image Quant, Microsoft Office, Adobe Illustrator, Adobe Photoshop, Endnote, GraphPad Prism, ChemBioDraw, Zeiss Axio imager, MVX Cell Sens

PRECLINICAL

Blast injury, Embolic Stroke, Animal behavior, Drug injection, Cardiac perfusion, Femoral \& tail vein injection, Stereotactic surgery, Embryo extraction, Microwave irradiation

LABORATORY

Western blot, RT-qPCR, Patch clamp, Cell culture, Ultrastructure 3D reconstruction, Immunohistochemistry, Microvessel isolation, Brain microdissection, Microtome \& Cryostat tissue preparation, Zeiss Confocal Microscopy

PROFESSIONAL EXPERIENCE

GUEST RESEARCHER | James P O'Callaghan PhD

NATIONAL INSTITUTE of OCCUPATIONAL SAFETY and HEALTH

Fall 2012-Present

INVITED SPEAKER | "The long-term consequences of multiple concussions"

Fall 2015

NATIONAL SCIENCE FOUNDATION | Oglebay Resort, Wheeling, WV

INVITED SPEAKER | "The unfolded protein response after traumatic brain injury"

Fall 2014

CENTERS for NEUROSCIENCE RETREAT | Oglebay Resort, Wheeling, WV

INVITED SPEAKER | "Neuropsychiatric symptoms of traumatic brain injury" MEETING of BEHAVIORAL and BIOMEDICAL RESEARCHERS | West Virginia University

Spring 2014

INVITED SPEAKER | "Endoplasmic reticulum stress and neurodegenerative disease"

Fall 2013 AMERICAN ASSOCIATION of PHARMACEUTICAL SCIENTISTS | Duquesne University

INVITED SPEAKER | "Quantitative assessment of stroke: home rehabilitation"

Spring 2012 LINKING INNOVATION INDUSTRY and COMMERCIALIZATION | Waterfront Plaza, Morgantown, WV

\section{LEADERSHIP}

AMERICAN AsSOCIATION of PHARMaceUtiCAL SCIENTISTS, Morgantown, WV VICE CHAIR

Allegheny College Theta Chi Alumni Association, Meadville, PA VICE PRESIDENT

DAVIES COMMUNITY SERVICE LEADER, Meadville PA SERVICE LEADER

Allegheny College Theta CHI Fraternity, Meadville, PA PRESIDENT $\mid$ TREASURER

The COUNTRY Club, Meadville, PA SUPERVISOR
Spring 2015-Present

Fall 2012-Fall 2016

Spring 2009-Spring 2010

Spring 2007-Fall 2009

Fall 2006-Fall 2011 


\section{GRADUATE COURSEWORK}

Molecular Cell Biology

Fundamental Integrated Systems

Molecular Genetics

Graduate Neuroscience
Stroke: Bench to Bedside

Human Physiology

Advanced Pharmacology 2

Scientific Writing
Programming Python

(Online; Michigan University)

Scientific Writing

(Online; Stanford University)

PUBLICATIONS

1. Logsdon AF, Lucke-wold BP, Nguyen L, Matsumoto RR, Turner RC, Rosen CL, Huber JD. 2016 Salubrinal reduces neuroinflammation and risk-taking behavior in a rodent model of traumatic brain injury. Exp Neurol (Submitted)

2. Lucke-wold BP, Nguyen L, Turner RC, Logsdon AF, Chen YW, Smith KE, Huber JD et al. 2015 Traumatic brain injury and epilepsy: Underlying mechanisms leading to seizure. Seizure [Epub]

3. Turner RC*, Lucke-Wold BP*, Logsdon AF*, Robson MJ, Lee J, Bailes J, Dashnaw M et al. 2015 Modeling chronic traumatic encephalopathy: the way forward for future discovery. Front Neurol [Epub] *Co-First Author

4. Turner RC*, Lucke-Wold BP*, Logsdon AF*, Robson MJ, Dashnaw M, Huang JH, Smith KE et al. 2015 The Quest to Model Chronic Traumatic Encephalopathy: A Multiple Model and Injury Paradigm Experience. Front Neurol [Epub] *Co-First Author

5. Lucke-Wold BP, Naser ZJ, Logsdon AF, Turner RC, Smith KE, Robson MJ, Bailes JE et al. 2015 Amelioration of nicotinamide adenine dinucleotide phosphate-oxidase mediated stress reduces cell death after blast-induced traumatic brain injury. Transl Res [Epub]

6. Logsdon AF, Lucke-Wold BP, Turner RC, Huber JD, Rosen CL, Simpkins JW. 2015 Role of microvascular disruption in brain damage from traumatic brain injury. Comp Phys 5:1147-60

7. Lucke-wold BP, Turner RC, Logsdon AF, Nguyen L, Bailes JE, Lee JM, Robson MJ et al. 2015 Endoplasmic reticulum stress implicated in chronic traumatic encephalopathy. J Neurosurgery [Epub]

8. Lucke-wold BP, Smith KE, Nguyen L, Turner RC, Logsdon AF, Jackson GJ, Huber JD et al. 2015 Sleep disruption and the sequelae associated with traumatic brain injury. Neurosci Biobehav Rev [Epub]

9. Tan Z, Lucke-wold BP, Logsdon AF, Turner RC, Tan C, Li X, Hongpaison J et al. 2015 Bryostatin extends tPA time window to 6 hours following middle cerebral artery occlusion in aged female rats. Eur J Pharmacol [Epub]

10. Logsdon AF, Turner RC, Lucke-wold BP, Robson MJ, Smith KE, Naser ZJ, Tan Z, Matsumoto RR et al. 2014 Altering endoplasmic reticulum stress in a model of blast-induced traumatic brain injury controls cellular fate and ameliorates neuropsychiatric symptoms. Front Cell Neurosci [Epub]

11. Lucke-Wold BP, Logsdon AF, Smith KE, Turner RC, Alkon DL, Tan Z, Naser ZJ et al. 2014 Bryostatin-1 restores blood-brain barrier integrity following blast-induced traumatic brain injury. Mol Neurobiol [Epub]

12. Lucke-Wold BP*, Logsdon AF*, Turner RC, Rosen CL, Huber JD, 2014 Aging, the metabolic syndrome, and ischemic stroke: redefining the approach for studying the blood-brain barrier in a complex neurological disease. Adv Pharmacol [Epub] *Co-First Author

13. Lucke-Wold BP, Turner RC, Logsdon AF, Simpkins JW, Alkon DL, Smith KE, Chen YW et al. 2014 Common mechanisms of Alzheimer's disease and ischemic stroke: the role of protein kinase $C$ in the progression of age-related neurodegeneration. J Alzheimers Dis 43:711-724. 
14. Tan Z, Li X, Turner RC, Logsdon AF, Lucke-Wold B, Jeong SS, Rosen CL et al. 2014 Combination treatment of $r$ - $t P A$ and an optimized human apyrase reduces mortality rate and hemorrhagic transformation 6 h after ischemic stroke in aged female rats. Eur J Pharmacol [Epub]

15. Logsdon AF, Lucke-Wold BP, Rosen CL, Huber JD 2014 Disparity among neural injury models and the unfolded protein response. J Neurol Disord Stroke [Epub]

16. Lucke-wold BP, Logsdon AF, Nguyen L, Lucke-wold AN, Turner RC, Huber JD, Rosen CL. 2014 Metabolic syndrome and its profound effect on prevalence of ischemic stroke. AMSRJ [Epub]

17. Lucke-wold BP, Turner RC, Logsdon AF, Bailes JE, Huber JD, Rosen CL. 2014 Linking traumatic brain injury to chronic traumatic encephalopathy: identification of potential mechanisms leading to neurofibrillary tangle development. J Neurotrauma [Epub]

18. Tan Z, Turner RC, Leon RL, Li X, Hongpaisan J, Zheng W, Logsdon AF et al., 2013 Bryostatin improves survival and reduces ischemic brain injury in aged rats after acute ischemic stroke. Stroke 44:3490-3497.

19. Turner RC, Naser ZJ, Logsdon AF, Jackson GJ, Robson MJ, Gettens RT, Huber JD et al. 2013 Modeling clinically relevant blast parameters based on scaling principles produces functional \& histological deficits in rats. Exp Neurol 248:520-529.

20. Turner, RC, Lucke-Wold, B, Lucke-Wold, N, Elliott, AS, Logsdon, AF, Rosen, CL, Huber, JD. 2013 Neuroprotection for ischemic stroke: moving past shortcomings and identifying promising directions. Int J Mol Sci 14:1890-1917.

\section{REFERENCES}

DR JASON D HUBER Professor of Pharmaceutical Sciences (Mentor)

304-293-1474 jdhuber@hsc.wvu.edu

DR CHARLES L ROSEN Chair of Neurosurgery (Co-mentor)

304-598-6127 crosen@hsc.wvu.edu

DR PAUL R LOCKMAN Chair of Pharmaceutical Sciences (Co-advisor)

304-293-0944 prlockman@ hsc.wvu.edu

DR JAMES W SIMPKINS Director of Stroke Research (Co-advisor)

304-293-7430 jwsimpkins@ hsc.wvu.edu 